\title{
Copyright
}

by

Garrett Randall Lambert

2004 
The Dissertation Committee for Garrett Randall Lambert Certifies that this is the approved version of the following dissertation:

\section{A TABU SEARCH APPROACH TO THE STRATEGIC AIRLIFT PROBLEM}

Committee:

J. Wesley Barnes, Supervisor

Leon S. Lasdon

John J. Hasenbein

Erhan Kutanoglu

Christopher Bassham 


\title{
A TABU SEARCH APPROACH TO THE STRATEGIC AIRLIFT PROBLEM
}

\author{
by \\ Garrett Randall Lambert, B.S., M.S.I.E.
}

\author{
Dissertation \\ Presented to the Faculty of the Graduate School of \\ The University of Texas at Austin \\ in Partial Fulfillment \\ of the Requirements \\ for the Degree of
}

Doctor of Philosophy

The University of Texas at Austin

December, 2004 


\section{Dedication}

To Bea, Connor and Gavin 


\section{Acknowledgements}

I would like to thank my advisor Dr. J. Wesley Barnes for his encouragement, insight and, above all, his patience. His unwavering support and understanding helped make this dissertation possible. To the gang who made up the "War Room" at UT, my thanks for making the abstruse clear and the hard times fun. To my parents, Harry and Joan Lambert, thank you, as always for your support and belief in me. To my siblings, Heather and Priscilla, thanks for your words of encouragement, humor and commiseration on coding and dissertation woes. To my in-laws, Harry and Josephine Nieder, thanks for your support and prayers. Pop, I know you did not live to see this completed but I can now report "Mission accomplished, Sergeant Major." To my two wonderful sons, Connor and Gavin, thanks for putting up with my absences from so many of the great things you do. I am so proud of you both. To my wife Bea, my rock and best

friend, all my love and thanks for your patience and support. Your selfless efforts to hold things together at home while managing a "day job" in the Army still leaves me in awe. I will never forget your sacrifice. 


\title{
A TABU SEARCH APPROACH TO THE STRATEGIC AIRLIFT PROBLEM
}

\author{
Publication No.
}

Garrett Randall Lambert, Ph.D.

The University of Texas at Austin, 2004

Supervisor: J. Wesley Barnes

Air Mobility Command (AMC) planners currently use simulation systems or large-scale linear programming (LP) models in studying the strategic airlift problem. Simulations are descriptive in nature and therefore cannot prescribe optimal flight schedules. Aggregation is used in large-scale LP models to make the problem tractable and thus much operational level detail is lost. AMC planners need a tool which prescribes good solutions while maintaining the operational level detail necessary to produce flight schedules. This research outlines a robust algorithm that obtains excellent solutions to the strategic airlift problem that possess the operational level detail necessary for AMC planners to develop the detailed routing and scheduling of strategic airlift aircraft. The algorithm utilizes the tabu search methodology. 


\section{Table of Contents}

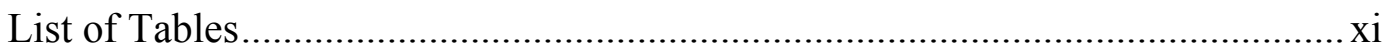

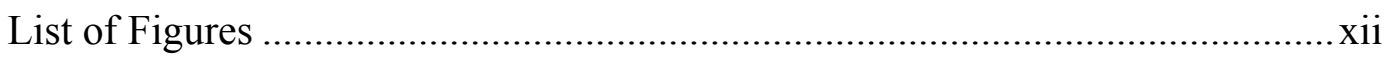

Chapter 1: Introduction ....................................................................... 1

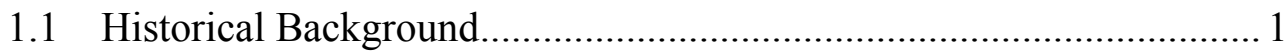

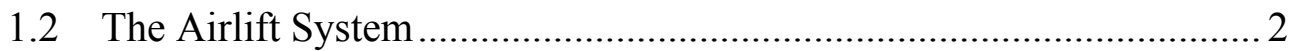

1.3 The Strategic Airlift Network ………………................................. 4

1.4 Strategic Airlift Modeling Interest .................................................. 8

1.4.1 Strategic Environment Impact................................................. 8

1.4.2 Current Strategic Airlift Models ................................................ 10

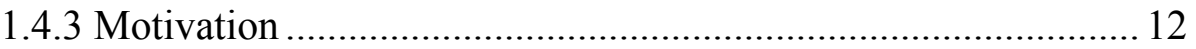

1.5 Research Objective........................................................................ 13

Chapter 2: $\quad$ Literature Review .................................................................... 15

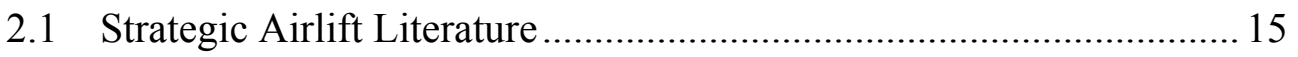

2.2 Strategic Airlift as a General Vehicle Routing Problem ....................... 19

2.3 Solution Approaches to General Vehicle Routing Problems .............. 23

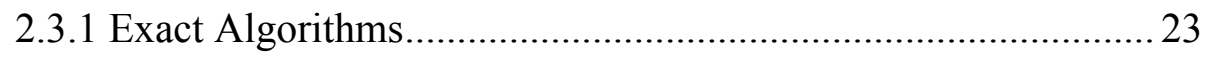

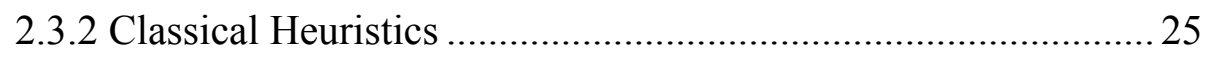

2.3.2.1 Constructive Heuristics ............................................... 25

2.3.2.2 Improvement Heuristics ............................................... 26

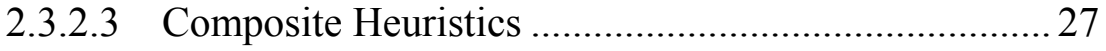

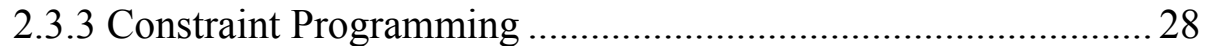

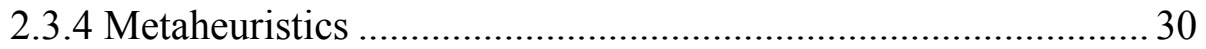

2.3.4.1 Simulated Annealing .................................................... 30

2.3.4.2 Genetic Algorithms ..................................................... 31

2.3.4.3 Tabu Search................................................................ 32

2.3.4.4 Tabu Search Variations ................................................. 33 
2.3.4.5 Tabu Search Applications to the GVRP ..................... 36

2.3.4.6 Adaptive Tabu Search Approach to the SAP ............. 38

Chapter 3: $\quad$ Detailed Problem Statement....................................................... 41

3.1 The Strategic Airlift Problem......................................................... 41

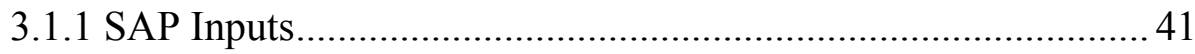

3.1.1.1 Requirements................................................. 41

3.1.1.2 Aircraft .......................................................... 43

3.1.1.3 Locations ........................................................ 45

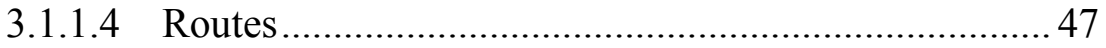

3.1.2 Additional SAP Characteristics.............................................50

3.1.2.2 A Standard Flight Leg ........................................... 50

3.1.2.3 Calculating Maximum Cargo for a Route ................... 51

3.1.2.4 Calculating Fuel Requirements for a given payload ... 59

3.1.2.5 Determining Aircraft Payloads................................. 60

3.1.3 SAP Building Blocks: Missions ........................................... 63

3.1.3.1 Determining Timings for a Mission .......................... 64

3.1.3.2 An Example Mission .................................................66

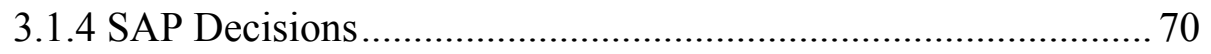

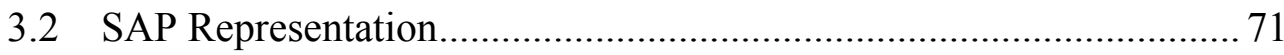

3.2.2 Pure Node Based Approach ................................................... 72

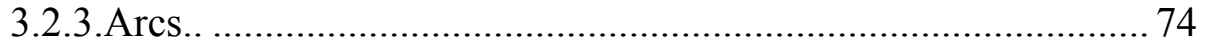

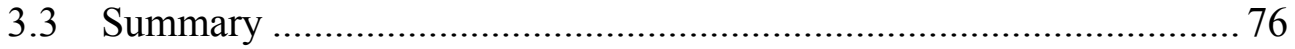

Chapter 4: $\quad$ A Tabu Search Approach to the Strategic Airlift Problem ........ 77

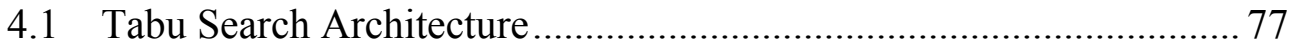

4.1.1 JAVA ${ }^{\mathrm{TM}}$ Software Programming Language and OpenTS ........ 77

4.2 SAP-TS Data Structures............................................................. 79

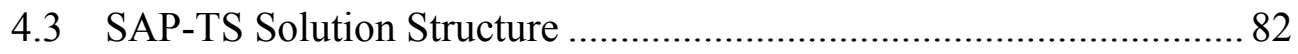

4.4 Initial Solution Construction ......................................................... 83 


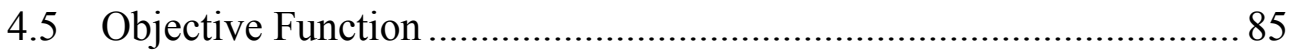

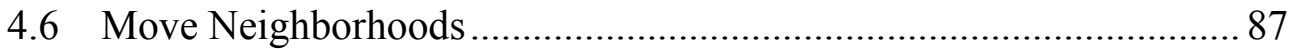

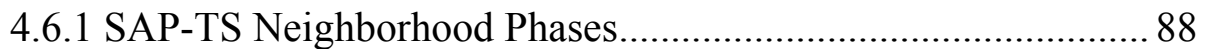

4.6.2 New Mission Insert Move Neighborhood (NMI) ........................ 89

4.6.3 Between Pair Swap Move Neighborhood (BPS) …………….... 92

4.6.4 Within Pair Insert Move Neighborhood (WPI).......................... 94

4.6.5 Between Pair Insert Move Neighborhood (BPI) ......................... 97

4.6.6 Within Mission Arc Swap Move Neighborhood (WMAS) ....... 98

4.6.7 Load Reallocation Move Neighborhood (LR) ............................. 99

4.6.8 Recover to APOE Arc Insert Move Neighborhood (RAI) ....... 100

4.6.9 Impose Time Delay Move Neighborhood (ITD) ...................... 101

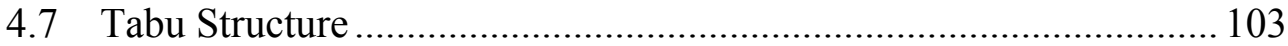

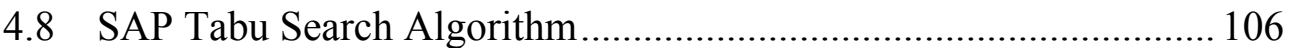

4.9 Mission Assignment/Scheduler Heuristic ............................................ 110

4.10 Move Manager .............................................................................. 114

4.11 An Iteration in the SAP-TS Algorithm ......................................... 117

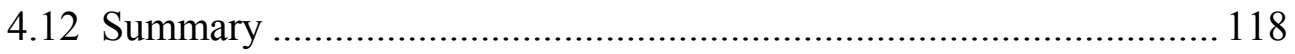

Chapter 5: An Application of the SAP Tabu Search Algorithm................. 120

5.1 SAP Problem Instances ...................................................................... 120

5.2 An Example Strategic Airlift Problem ............................................. 121

5.2.1 Scenario 5 Details.................................................................. 122

5.2.2 SAP-TS Results for Example Problem ..................................... 125

5.2.3 AMOS Results for Example Problem ..................................... 128

5.3 Other SAP Problem Instances ……………………......................... 130

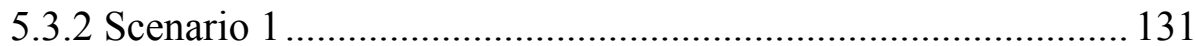

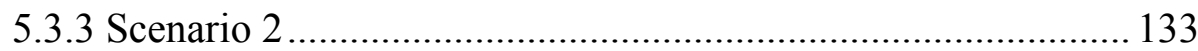

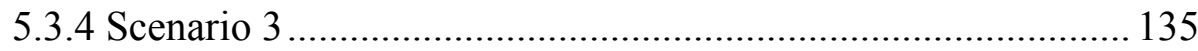

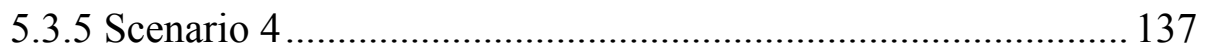

5.4 Extended Analysis of Scenario 2 .................................................... 139 


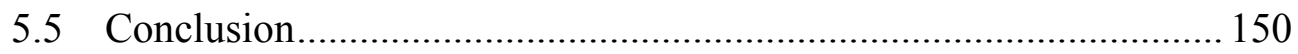

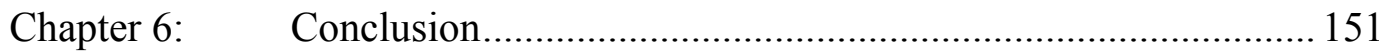

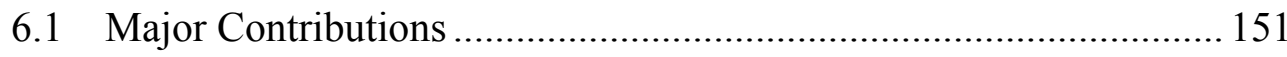

6.2 Further Enhancements to the SAP-TS Algorithm.............................. 152

6.3 Extensions to other aspects of the SAP ………………………......... 154

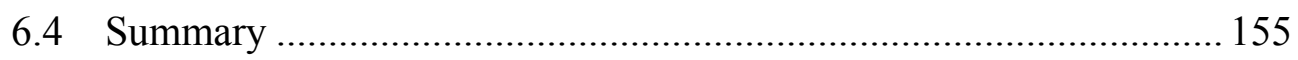

Appendix A: SAP-TS Best Solution Scenario Mission Summaries ................... 157

A.1 Scenario 1 Best Solution Mission Summary ..................................... 157

A.2 Scenario 2 Best Solution Mission Summary ……………………....... 161

A.3 Scenario 3 Best Solution Mission Summary ....................................... 170

A.4 Scenario 4 Best Solution Mission Summary ........................................ 177

A.5 Scenario 5 Best Solution Mission Summary ....................................... 181

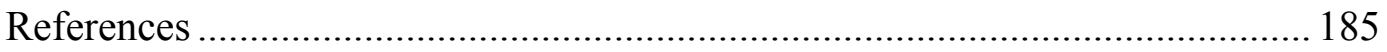

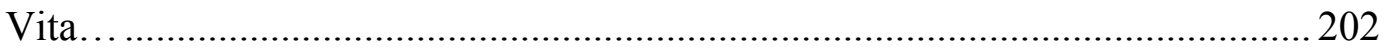




\section{List of Tables}

Table 3.1 Route Segment Details for KGRK - OKBK Route.....................52

Table 3.2 C-5A Standard Weights and Fuel...........................................53

Table 4.1 SAP-TS Objects and their Attributes ..................................... 80

Table 4.2 Aircraft and Missions Available ............................................ 90

Table 4.3 APOE-APOD Pairs and Assigned Missions .............................91

Table 4.4 APOE Arrival Times ............................................................ 95

Table 4.5 Tabu Search Parameters ........................................................... 108

Table 4.6 Problem Instance Parameters ................................................... 109

Table 4.7 Arcs Connecting KDOV to OKBK ......................................... 112

Table 4.8 Neighborhood Selection After Phase Change .......................... 117

Table 5.1 Scenario Characteristics ......................................................... 121

Table 5.2 Example Problem Available Aircraft ...................................... 122

Table 5.3 Example Problem Requirements ............................................ 123

Table 5.4 Example Problem Route Segments ...................................... 125

Table 5.5 Example Problem Tabu Search Settings .................................. 126

Table 5.6 Example Problem Initial Solution Objective ........................... 127

Table 5.7 Example Problem Best Solution Objective ............................. 128

Table 5.8 Example Problem AMOS \& SAP-TS Objective........................ 129

Table 5.9 AMOS and SAP-TS Results for Scenarios 1 through 4.......... 131

Table 5.10 Number of Required C17 Missions by Day for Scenario 2 .... 142

Table 5.11 Scenario 2 Aircraft Arrivals by Type.................................... 145

Table 5.12 Scenario 2 Working MOG Violation Bases (SAP-TS) ............ 147

Table A.1 Scenario 1 Mission Summary ................................................ 161

Table A.2 Scenario 2 Mission Summary ................................................. 169

Table A.3 Scenario 3 Mission Summary ….......................................... 177

Table A.4 Scenario 4 Mission Summary ............................................. 181

Table A.5 Scenario 5 Mission Summary ............................................. 184 


\section{List of Figures}

Figure 1.1

Figure 1.2

Figure 3.1

Figure 3.2

Figure 3.3

Figure 3.4

Figure 3.5

Figure 3.6

Figure 3.7

Figure 3.8

Figure 3.9

Figure 3.10

Figure 3.11

Figure 3.12

Figure 3.13

Figure 3.14

Figure 3.15

Figure 4.1

Figure 4.2

Figure 4.3

Figure 4.4

Figure 4.5

Figure 4.6

Figure 4.7

Figure 5.1

Figure 5.2

Figure 5.3

Figure 5.4

Figure 5.5

Figure 5.6

Figure 5.7

Figure 5.8

Figure 5.9

Figure 5.10

Figure 5.11

Figure 5.12

Figure 5.13

Figure 5.14

Figure 5.15
Airlift Mission Classifications (AFDD 2-6.1 1999)..................... 3

Airlift Network Example........................................................... 7

Extract from AMOS Payload Target File for C-5A …................ 42

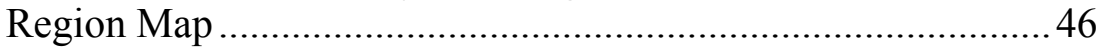

Route Segment Extract from AMOS Plan File .......................... 48

Standard Flight Leg with Segments ........................................5 50

Climb Data Extract from fuelcalc. dat File for a C-5A...............54

Cruise Data Extract from fuelcalc.dat file for a C-5A ................ 55

Example Maximum Cargo Calculation for a C-5A .................. 59

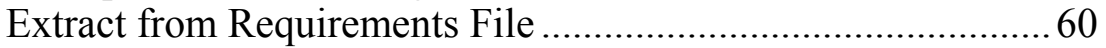

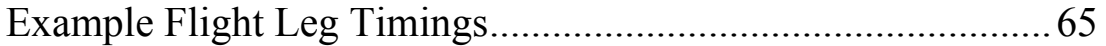

Route Segment Joining Region 21 to Region 20 ......................6 67

Extract from Payload Target File for C-5As .............................. 68

Route Segment for OKBK to LEMO ........................................69

Route Segment for LEMO to KDOV ....................................... 70

SAP Representation using Pure Nodal Approach ...................... 72

SAP Representation using Arcs.............................................. 75

OpenTS Required Elements and Associated SAP-TS Classes .. 78

OpenTS Architecture (Harder 2000) ........................................ 79

Pseudo Code for Initial Solution ............................................... 84

WPI Insert Points for Mission 0 ............................................... 97

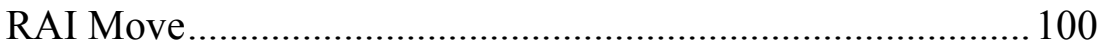

Mission Assignment/Scheduler Heuristic Pseudo Code .......... 111

Determination of Neighborhood Phase and Search Context.... 115

Example Problem Requirements and Aircraft Available......... 124

Example Problem PAX \& Cargo Closure ................................ 130

Scenario 1 Total Daily Requirements and Aircraft Available. 132

Scenario 1 PAX \& Cargo Closure.............................................. 133

Scenario 2 Total Daily Requirements and Aircraft Available .134

Scenario 2 PAX \& Cargo Closure........................................... 135

Scenario 3 Total Daily Requirements and Aircraft Available . 136

Scenario 3 PAX \& Cargo Closure........................................... 137

Scenario 4 Total Daily Requirements and Aircraft Available . 138

Scenario 4 PAX \& Cargo Closure............................................. 139

SAP-TS Scenario 2 Solution with Time Windows ................... 140

C17 Missions by Time Window and APOE-APOD Pair......... 143

SAP-TS Missions vs C17 Equivalent Missions ....................... 145

AMOS Missions vs C17 Equivalent Missions ......................... 146

SAP-TS Working MOG for OKBK, LEMO and LICZ ........... 149 


\section{Chapter 1: Introduction}

Airlift is the transportation of personnel and materiel through the air and can be applied across the entire range of military operations in support of national objectives.... A key function of the Air Force, airlift provides global reach for US military forces and the capability to quickly apply strategic global power to various crisis situations worldwide by delivering necessary forces.

Air Force Doctrine Document 1: Air Force Basic Doctrine

This dissertation describes an adaptive tabu search (ATS) approach to the Air Mobility Command's strategic airlift problem (SAP). To facilitate that description, the next section provides a brief historical background of the SAP followed by descriptions of the airlift system and the strategic airlift network.

\subsection{HISTORICAL BACKGROUND}

Historically, strategic lift (airlift and sealift) has been seriously considered only in the crucible of war. Strategic airlift, the more responsive of the two, has fared no better than sealift. Because of the large, time-sensitive mobilization requirement placed upon the airlift network, the strategic airlift fleet often finds itself in the unenviable position of playing catch-up at the start of conflict. The U.S. Army championed initial interest in strategic airlift in the mid-1930's with the purchase of airplanes designed specifically to transport cargo (Callander, 1998). At the start of World War II, a mere 254 of the 12,297 aircraft in the Air Corps were for cargo transport. By the end of the war, strategic airlift was firmly established as a key component of U.S. air doctrine. 
Throughout the Cold War and in recent times, strategic airlift has continued to play a pivotal role in the support of national military strategy. The Berlin Airlift is perhaps one of the most striking examples of the efficacy of strategic airlift in the furtherance of U.S. objectives. By the end of the Korean and Vietnam wars, strategic airlift assets were organized under a single command, the Military Airlift Command (later transformed into Air Mobility Command), and included aircraft from the Civil Reserve Air Fleet (CRAF). The buildup of forces for Operation Desert Storm stretched air mobility capabilities to the limit and represented the "most massive airlift in the history of airpower" (Callander, 1998). Recent operations in Afghanistan mark the first time in US history in which the United States moved its war machine overseas exclusively by air (Loeb, 2002).

Over its varied history, strategic airlift has become many things--simple cargo transport, airborne drops, glider support, medical evacuation, and sophisticated aerial resupply methods, to name only a few. Today, it is an indispensable component of national military power. It is imperative to carefully manage and efficiently utilize available strategic airlift resources so that US air mobility capabilities will be equal to the daunting tasks of the future.

\subsection{THE AIRLIFT SYSTEM}

Air mobility is composed of airlift, air refueling, and air mobility support - the air mobility triad (AFDD 2-6, 1999). Air mobility forces are drawn from active duty, Air Force Reserve Command (AFRC), Air National Guard (ANG), and Civil Reserve Air Fleet (CRAF) components. The active duty 
component is the most responsive and flexible. AFRC and ANG, when mobilized, are equivalent to the active component. CRAF provides important surge capacity during contingency and wartime operations. In return for this service, CRAF participating carriers are contracted for movement of passengers and cargo during peacetime (AFDD 2-6.1, 1999). Together, these components provide the aircraft, crews and support forces that make up the airlift system.

The airlift system pictured in Figure 1.1 is an integrated hierarchical system that consists of intertheater, intratheater, and dedicated Joint Task Force (JTF) airlift (AFDD 2-6.1, 1999).

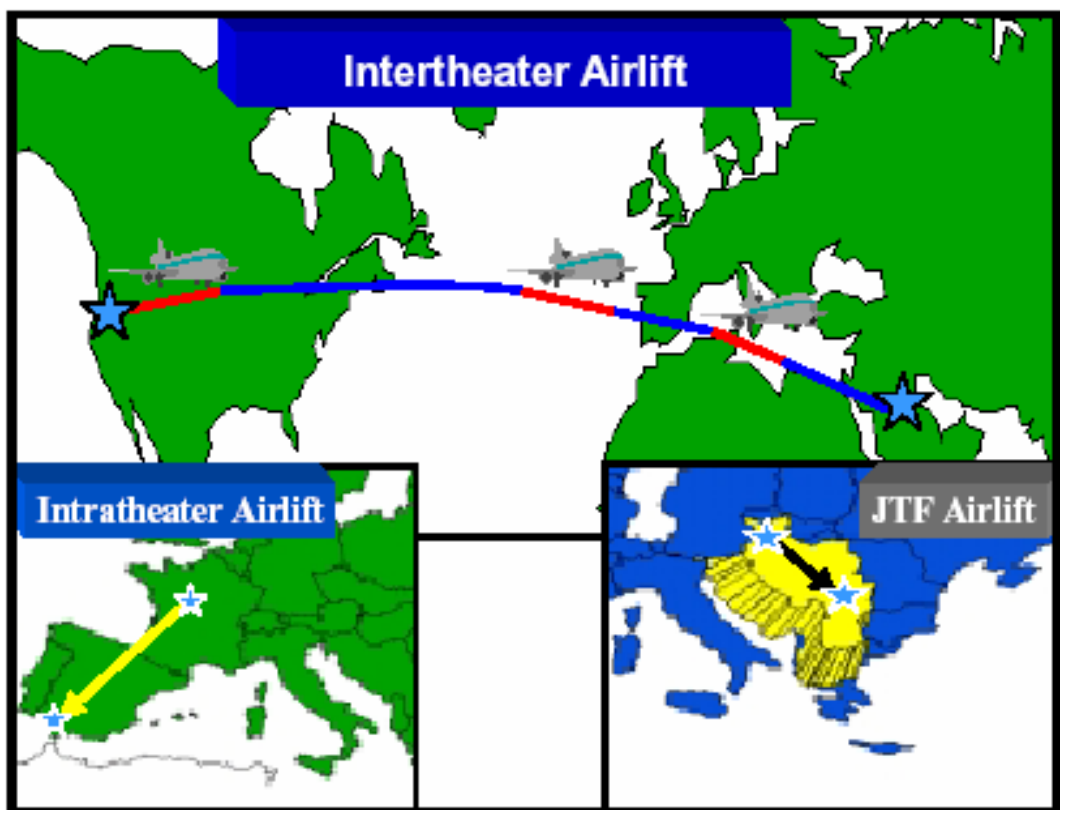

Figure 1.1 Airlift Mission Classifications (AFDD 2-6.1 1999)

The airlift system delivers personnel and cargo when and where they are needed. Airlift operations can be characterized as intertheater airlift, intratheater airlift, or Operational Support Airlift (OSA). Intertheater airlift provides airlift 
"linking theaters to the continental United States (CONUS) and to other theaters as well as the airlift within CONUS. These airlift assets are assigned to the Commander in Chief, United States Transportation Command" (AFDD 2-6.1 1999). Since typical intertheater distances are large, strategic airlift is normally comprised of the heavy, longer range, intercontinental airlift assets. Intratheater airlift is "that airlift assigned or attached to a combatant commander other than Commander in Chief, US Transportation Command, which provides air movement and delivery of personnel and equipment directly into objective areas ...to meet specific theater objectives and requirements" (AFDD 2-6.1 1999). OSA operations are time-sensitive movements of high-priority personnel and small amounts of cargo.

The problem of scheduling intertheater airlift operations, the SAP, is the focus of this research.

\subsection{The STRATEgIC Airlift Network}

The SAP is primarily concerned with intertheater airlift of personnel and cargo from CONUS to the theater of operations or from one theater to a different theater. The mission of the US Transportation Command (USTRANSCOM) is to provide air, land, and sea transportation for the Department of Defense (DOD) in peace and in war. Air Mobility Command is the USTRANSCOM component responsible for conducting airlift operations (AMC).

At the outset of a contingency operation or war, USTRANSCOM receives personnel and equipment requirements from the Time Phased Force Deployment Document (TPFDD) developed by the combatant forces. The TPFDD specifies 
such details as the personnel and equipment to be moved, their origin and destination locations, when they are available for loading at origin and when they are required to be delivered at their destination. Once USTRANSCOM determines the mode (air or sea) of transport, requirements are then distributed to the appropriate subordinate commands in USTRANSCOM--AMC for airlift requirements and Military Sealift Command (MSC) for sealift requirements. Planners at AMC then establish an airlift network to meet the movement requirements defined by USTRANSCOM. The airlift network consists of aircraft, aircrews, airfields, and support equipment and personnel.

The amount and type of aircraft employed and the airfields used are a function of the airlift requirements derived from the TPFDD. Sources for aircraft may be either military or CRAF. The aircraft employed can be cargo and/or passenger aircraft. The airfields employed in the network include the origin and destination airfields outlined in the TPFDD for each requirement. Origin airfields are aerial ports of embarkation (APOEs). At the APOEs, personnel and equipment are loaded onto aircraft. For the SAP, APOEs typically are located within CONUS, where the bulk of US forces are stationed. Personnel and equipment are unloaded at the destination airfields, aerial ports of debarkation (APODs). The APODs are generally located within or near the theater of operations. Because the large distances between APOEs and APODs generally preclude direct flights, $\mathrm{AMC}$ incorporates a series of en route bases into the network that service the various APOE-APOD pairs. En route bases can be selected from existing bases or established for a particular contingency using air 
mobility support forces. These en route bases are used for refueling of aircraft, changing and staging of aircrews and performing required aircraft maintenance. The number and location of en route bases utilized are determined by the APOEAPOD pairs that need to be serviced and the number of aircraft available. There is a special virtual en route base called a waypoint that may also be established in defining the airlift network. Waypoints are points in space through which an aircraft must fly. Among other uses, waypoints serve as rendezvous points for aerial refueling and as additional control measures to further specify a route (e.g., route aircraft around restricted airspace).

Crews are not assigned to a particular aircraft. Rather, aircraft move continuously through the airlift network changing crews at en route bases as necessary. A typical mission cycle for an aircraft starts at its home base (the aircraft's permanent base) with the initial aircrew. The aircraft is then flown to an APOE where some portion of the requirement (cargo and/or passengers) is loaded. The aircraft is then flown along a route leading to the APOD, passing through a series of en route bases along the way to refuel, make repairs or change crews as necessary. Ultimately, the aircraft reaches the APOD and the cargo and/or personnel are unloaded. The aircraft is then flown to a recovery base for any required maintenance and crew change. At this point, the aircraft may return to its home base or it may fly to another APOE for the start of a new mission. In either case, the aircraft will again pass through a series of en route bases for refueling, repair and crew changes as necessary. For the SAP, aircrews are, in general, not the bottleneck in the system. There is roughly a three to one ratio of 
aircrews for military aircraft. Therefore, once aircraft and requirements are scheduled, the crew scheduling problem can be solved independently.

A key constraint on the flow through the airlift network is the limited capacity of the various airfields to service arriving aircraft. Among the finite resources available for servicing aircraft are parking spaces, material handling equipment (MHE), available fuel, and support personnel. To simplify modeling this constraint, AMC planners use an aggregate number called "maximum-on-theground" (MOG) to capture the number of aircraft that can be simultaneously serviced (worked) or parked or refueled at the airfield.

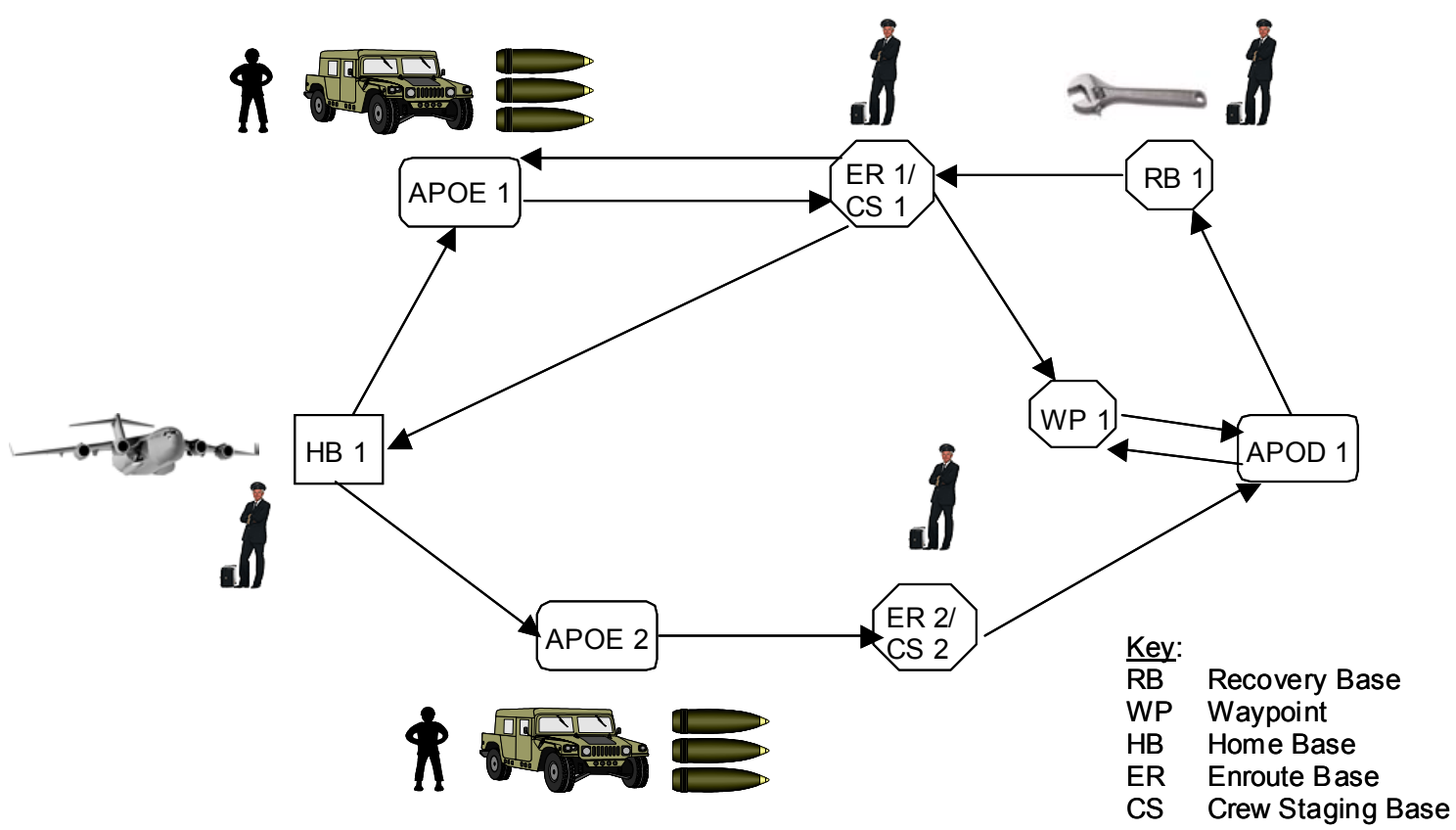

Figure 1.2 Airlift Network Example

Working MOG is the number of aircraft that can be simultaneously "worked" using MHE, servicing equipment, and support personnel so that all the 
aircraft can leave the airfield within their allotted ground times. Parking MOG refers to the maximum number of aircraft that can be accommodated on the airfield. Finally, fuel MOG is the maximum number of aircraft that can be fueled simultaneously. Typically, working MOG is more restrictive than parking MOG and fuel MOG. An example of an airlift network is depicted in Figure 1.2.

\subsection{Strategic Airlift Modeling InTEREST}

\subsubsection{Strategic Environment Impact}

History is replete with examples of the tremendous impact strategic airlift has had upon the accomplishment of national policy and objectives. Notable examples of the efficacy and importance of strategic airlift include the Berlin Airlift, support of Operations Desert Shield/Storm, and most recently the support of the war effort in Afghanistan and Iraq. In each of these cases, strategic airlift has played a significant role in the enhancement of US national objectives (Rower, 2001). Three primary factors influence strategic airlift today and create a need for the efficient use of air mobility assets.

The first of these factors is the posture of US military forces. Over the last decade, the US military has de-emphasized forward deployment and has emphasized force projection. As a result, the ability to rapidly project large numbers of forces, strategic mobility, is even more critical. Air mobility is the most flexible and responsive means to rapidly project and sustain combat forces during peace and war. 
A projected shortfall in military airlift capability is the second factor influencing the strategic airlift. In 1998, the Department of Defense initiated a comprehensive two-year study, the Mobility Requirements Study-2005 (MRS05), to determine strategic mobility requirements based on 2005 force structure. For strategic airlift, the accepted measure of capacity to deliver cargo (in tons) through a distance (in miles) in a period of time (in days) with a given fleet of vehicles is million ton-miles per day (MTM/D). MRS-05 predicts that US strategic airlift requirements will exceed the current airlift capability of 49.7 MTM/D by more than $5 \mathrm{MTM} / \mathrm{D}$. Procurement of additional aircraft, modernization of existing aircraft and improvements to base infrastructure are among the many strategies established to deal with this shortfall.

The third factor is the changing strategic environment. The US National Military Strategy (NMS) is evolving in response to the dynamic nature of global threats to US interests. US NMS mandates the ability to prosecute two nearly simultaneous major theater wars (MTWs). This presents a significant challenge for air mobility planners. Additionally, recent experience (e.g., Afghanistan) indicates that smaller scale contingencies (SSCs) are increasingly likely scenarios for military forces. SSCs are characterized by austere airfields and ports that limit the introduction of forces in theater. Thus, in this environment, even if the MTM/D requirement of above were met, infrastructure limitations could largely reduce the capacity of the airlift network. Thus SSCs, which generally have smaller requirements than an MTW, can still exert a major strain on the air mobility system. 
The US military's transition from forward deployment to force projection, the projected airlift shortfalls, and a dynamic strategic environment together place a premium on the importance of effective employment of air mobility assets. In recent testimony to the US Senate Armed Services Committee on US operations in Afghanistan, General Thomas Franks, commander of US Central Command, stated, "Strategic airlift remains key to current and future military operations. We are on a glide-path to expand our strategic airlift capabilities and must remain committed to the task."

\subsubsection{Current Strategic Airlift Models}

Over the years, AMC has implemented a number of models to assist planners in assessing a particular airlift network's capacity, mission planning, and in developing aircraft schedules. The focus here will be on models that are used as aids in the SAP. Many of these models are legacy systems that have been retained and improved upon over the years. As detailed below, AMC uses ADANS, CAMPS, AFM, ACAS, JFAST and AMOS in various contexts to arrive at a plan for a given TPFDD requirement. A brief synopsis of each of these models follows.

- The Airlift Deployment Analysis System (ADANS), developed by Oak Ridge National Laboratory, was implemented in 1990 to support the scheduling of airlift missions for Operations Desert Shield/Storm. ADANS was the first major effort to automate scheduling of strategic airlift. ADANS incorporates a dynamic programming approach along with a greedy heuristic that sequentially builds missions. In 2002, the Consolidated Air Mobility Planning System (CAMPS) replaced ADANS adding the aerial refueling problem solution to the SAP by joining ADANS 
with the Combined Mating and Ranging Planning System (CMARPS) for tanker scheduling.

- The Airlift Flow Model (AFM) is a legacy stochastic simulation model that incorporates stochastic elements such as winds and maintenance variability. AFM is a very complex, user-unfriendly model and is now obsolete.

- The Airlift Cycle Analysis Spreadsheet (ACAS) is a single cycle deterministic spreadsheet model used as a "quick look" tool. A major limitation of ACAS is that it is restricted to a single APOE/APOD pair.

- The Joint Flow Analysis System for Transportation (JFAST) is a dynamic programming based multimodal transportation analysis model designed for USTRANSCOM and implemented in 1991. It is used to determine transportation requirements, perform course of action analysis, and project delivery profiles of troops and equipment by air, land, and sea. AMC uses JFAST to analyze the airlift transportation requirements for deploying US forces and predict their arrival dates in-theater. JFAST can be used to determine the transportation feasibility of airlift deployment plans. Finally, JFAST may also be used as a TPFDD editor.

- The Air Mobility Operations Simulation (AMOS) is a developing stochastic simulation model that will eventually replace AFM. AMOS is being built to support in-depth analysis of global mobility requirements and deployment capabilities to include resource allocation, aircrew availability, route optimization, aerial refueling, and intra-theater airlift. AMOS is an ambitious effort that will attempt to tie all elements of AMC scheduling togetherairlift, air refueling, and air mobility support.

While these models address some of AMC's planning needs, they are incapable of obtaining effective detailed solutions to realistic instances of the SAP. ADANS/CAMPS are used primarily for deliberate planning at AMCplans that are developed before a particular crisis or contingency occurs. Because ADANS/CAMPS and JFAST are based upon a dynamic programming approach, 
they are particularly susceptible to the "curse of dimensionality." As a result, realistic problem sizes are reduced through aggregation and the use of a greedy myopic heuristics (for pairing aircraft to requirements) to reduce the solution space. Solutions obtained are feasible but not necessarily "good." Since ACAS is limited to a single APOE/APOD pair, it is not sufficient for obtaining solutions to realistic SAP instances.

Thus AFM (and later AMOS) is the primary tool used to solve the SAP. A major drawback of simulation models like AFM and AMOS is that they do not describe what is "best." Instead, these models enable analysts to investigate "what if" scenarios which, over a multitude of runs, can lead to insight into the airlift system. In the context of the SAP, AFM and AMOS proceed by loading cargo on the first available aircraft that is then routed according to a pre-selected prioritized list — a greedy myopic measure of goodness. Solutions obtained in this manner, while feasible, are generally not very good.

\subsubsection{Motivation}

Current models that incorporate simulations or traditional optimization methods do not provide, for a realistic problem size, a near-optimal combination of aircraft and routes for each requirement at a sufficient level of detail. AMC requires an AMOS compatible methodology that rapidly provides excellent solutions to realistic instances of the SAP with sufficient detail to build a mission schedule for each aircraft. The method must have the capability to address multicriteria objectives such as minimizing the time required for unit closure (delivery of a specified fraction of the unit to the APOD), minimizing aircraft 
utilization and maximizing cargo throughput while still meeting TPFDD delivery requirements.

Tabu search has been shown to be particularly effective in solving large scale combinatorial optimization problems related to the SAP. Recent work by Barnes, Wiley, Moore and Ryer (2004) on the Aerial Flight Refueling Problem (AFRP), Crino, Moore, Barnes and Nanry (2004) on the Theater Distribution Vehicle Routing and Scheduling Problem (TDVRSP), and Combs (2002, 2004) on the Crew Scheduling Problem (CSP) clearly demonstrate that a tabu search approach can provide robust, effective and efficient solutions to practical large scale combinatorial optimization problems. Thus an ATS approach to the SAP will provide an effective methodology to fill the gaps left by current models at AMC by providing timely, robust, detailed and excellent solutions to the SAP.

\subsection{RESEARCH OBJECTIVE}

The primary objective of this research is to develop a robust and effective solution methodology that solves the Strategic Airlift Problem. The solution will dictate the detailed assignment of cargo to aircraft and the routing and scheduling of aircraft missions through the network.

There are several supporting objectives for this research. The first supporting objective is to develop an effective and extensible representation of the SAP. Since the SAP instances are defined by the AMOS input files, the methodology must mirror many of the modeling aspects and problem structure imposed by AMOS. This representation captures essential aspects of the SAP yet remains tractable for application of the tabu search methodology. The SAP 
representation can be extended to include additional aspects of the SAP not incorporated in this research such as transloading operations.

A second supporting objective is to develop a tabu search algorithm that solves the SAP efficiently and effectively. The algorithm uses an adaptive tabu search approach incorporating effective neighborhoods and neighborhood selection schemes to provide the user with a suite of excellent solutions.

The third supporting objective is to code the SAP algorithm in the Java ${ }^{\mathrm{TM}}$ software programming language. The $\mathrm{Java}^{\mathrm{TM}}$ language portability and reuseability facilitates use of the software on different platforms and expansion of the software. Using Harder's (2000) tabu search architecture as the framework, the SAP software implements the SAP tabu search algorithm providing the user with detailed solutions to SAP instances. 


\section{Chapter 2: $\quad$ Literature Review}

\subsection{STRATEGIC AIRLIFT LiteratURE}

Surprisingly, despite the premium placed on airlift capabilities, there is little in the literature on the SAP. As discussed in Section 1.4.2, most of the work on the SAP at AMC has centered on the use of simulation models. However, exact approaches such as linear programming (LP) and hybrid approaches, that combine exact methods with heuristics, have also appeared in the SAP literature. The following is a summary of the pertinent work on the SAP in the literature.

The first major effort to automate the scheduling of strategic airlift, ADANS, was developed by Hilliard et al. (1992) of Oak Ridge National Laboratory just prior to Operation Desert Shield/Storm. ADANS, a hybrid method, incorporates a dynamic programming approach that uses a sequential procedure that schedules one mission at a time taking into consideration the constraints imposed by previously scheduled missions. Rule based heuristics are used to build and sort pairings of aircraft with requirements. A weighted objective function is used which considers minimization of such things as late deliveries, mission flying time, and the number of crew changes. There are several limitations to this model. Clearly, due to the scale of strategic airlift problems, a dynamic programming approach is particularly susceptible to the "curse of dimensionality." Additionally, the aircraft-requirement pairing heuristic is a greedy (local) approach that restricts, a priori, the network configurations that can be considered. Although ADANS is still in use at AMC today, its use is 
largely relegated to feasibility studies and force structure issues instead of actual scheduling of aircraft in a crisis.

Rappoport et al. (1992) developed a myopic greedy airlift planning heuristic (APH) for incorporation into ADANS that first assigns requirements to aircraft and then routes and schedules the aircraft. Captured in a "plane preference value," assignment of a requirement to an aircraft is based upon the idea of "best fit" in terms of the most constraining restriction associated with either weight, volume, or square feet. Rappoport et al. (1991) provide a detailed description of plane preference values. Once aircraft-requirement pairings are made, the heuristic attempts to obtain feasible routes (up to two for each aircraft) for up to five aircraft of the preferred type using a shortest path approach. Once feasible schedules are obtained, crew constraint feasibility is verified. Feasible schedules are assessed using a weighted objective function that incorporates total mission time, total on ground delays and time of delivery in excess of the RDD. The APH heuristic represented a significant improvement over the level of detail and the efficiency of methods in use by AMC at the time. However, it is limited by its greedy myopic underpinnings that significantly restrict the solution space.

There are several linear programming (LP) based models described in the literature that were implemented to support studies at levels above AMC such as at the USTRANSCOM or Joint Staff level. As an alternative or in concert with detailed simulations, LP can be used to answer many questions concerning the Air Mobility system. However, they do not to provide operational level detail, rather 
they provide insights into the airlift network. These models are generally built to assess more global considerations such as:

(1) Where are the bottlenecks in the airlift network?

(2) What is the best force structure (considering such things as fleet mix and airfield support team mix) given the requirements outlined in the National Military Strategy?

(3) What is the force closure capability for a given fleet of aircraft over a specified network?

These are broad level questions that are generally answered using broad models. As a consequence, key features of the problem are aggregated so that realistic instances can be addressed.

A significant amount of the literature on the SAP originates from the Naval Post Graduate School. Yost (1994) developed a flow optimization model in the General Algebraic Modeling System (GAMS) called THRUPUT. THRUPUT is a time static model of the SAP on a general routing network.

In Morton et al. (1996), several improvements to Yost's THRUPUT model are developed into a model called THRUPUT II. THRUPUT II formulates the SAP as a multi-period, multi-commodity network-based LP model that includes a number of side constraints such as aircraft \& airfield capacity constraints, aircraft utilization constraints, and aircraft balance constraints. To capture the time dynamic nature of the SAP, THRUPUT II makes use of a time index to track aircraft locations in each discretized time period. To overcome the intractability issue common with realistic SAP instances, an aggressive preprocessing step is 
used to reduce the model size through removal of unnecessary variables/constraints and data aggregation. Additionally, some key assumptions are made in THRUPUT II to reduce problem size and they include the aggregation of airfield capacity into working MOG and the use of deterministic ground times. THRUPUT II trades the high level detail and the inclusion of uncertainty of simulation models for the rapid answers to broad questions like those listed above. THRUPUT II was successfully used by the Air Force Studies and Analyses Agency (AFSAA) to analyze procurement issues for the C-17 Defense Acquisition Board (Rosenthal et al. 1997).

Baker et al. (2002) further enhance the THRUPUT II model by melding the best of THRUPUT II and a RAND Corporation model called CONOP (CONcept of Operations). Like THRUPUT II, CONOP is a time dynamic model on a general airlift network. Unlike THRUPUT II, CONOP includes aerial refueling, crew constraints, transloading options, and the use of recovery bases as an option. A key drawback of CONOP is that it does not have sufficient resolution of the cargo. The result of the marriage of THRUPUT II and CONP was NRMO (NPS/RAND Mobility Optimizer). As in THRUPUT II, NRMO incorporates aggregation to reduce problem size. Baker et al. report several key insights gained while using NRMO. Chief among these is the impact of winds on throughput. Since NRMO incorporates many of the major features of the SAP and is computationally efficient, it has been successfully used in a variety of analyses to assist Air Force planners in answering questions concerning force structure and airfield resources issues. 


\subsection{Strategic Airlift as a General Vehicle Routing Problem}

The General Vehicle Routing Problem (GVRP) is a useful paradigm for viewing the SAP. The GVRP is any problem that seeks to determine the best (as measured by some objective) routing of vehicles to satisfy a given set of customers. More fully, the GVRP can be expressed as follows: given a set of customers requiring a visit, and a fleet of vehicles based at a depot that can perform the visits, construct a set of routes for the vehicles that minimizes the costs of operation. The objective function is typically expressed as costs related to the number of vehicles and to distance traveled. Constraints include various capacity constraints on weight, volume, length; time constraints on when the customer will accept a visit; and the total length of routes. In practical problems there may be different kinds of additional constraints including legislative restrictions, established work practices and customer preferences, and a complex objective function reflecting complicated pay provisions.

The Traveling Salesman Problem (TSP), the Vehicle Routing Problem (VRP) and the Pickup and Delivery Problem (PDP) all fall within the GVRP class. Distinctions between classes are due to the manner in which vehicles, customer service, and costs are defined. For example, in the TSP, the problem is to find a route for a single vehicle (salesman) with unlimited capacity that visits (services) each customer once at least cost (generally measured as distance traveled). For the PDP, the vehicle has a finite capacity and services to customers entail precedence constraints that ensure that an item is picked up before it is delivered. Variations of the TSP, VRP and PDP are numerous and difficult to 
categorize. These variations include such considerations as multiple vehicles, multiple depots, time windows, and route length constraints that greatly increase the complexity of the GVRP.

Carlton (1995) extends the classification of vehicle routing and scheduling problems by Bodin et al. (1983) by providing a multi-tiered framework to characterize the GVRP hierarchy. In this hierarchy the TSP, VRP and PDP represent succeeding floors where the stairs from the TSP to the VRP are formed in the presence of capacity constraints and the stairs from the VRP to the PDP reflect the existence of precedence constraints. Within each floor, Carlton includes other typical elements of GVRPs such as:

1. Number of vehicles $-\mathrm{a}$ single vehicle (SV) or multiple vehicles (MV)

2. Type of vehicles-homogeneous (same type) vehicles $(\mathrm{H})$ or different types of vehicles $(\overline{\mathrm{H}})$

3. Number of depots - a single depot (SD) or multiple depots (MD)

4. Route Length (RL) constraints - distance or amount of time vehicles may travel

5. Time Windows (TW) — service for a customer must fall within a designated time window specified by an earliest and latest time to start a service

Using Carlton's GVRP classification hierarchy, the SAP can be partly classified as a MV $\overline{\mathrm{H}}, \mathrm{MD}, \mathrm{PDP}$, with RL and TW constraints. Crino (2002) extends Carlton's hierarchy by adding the following characteristics: single trip 
(ST) per vehicle versus multiple trip (MT) per vehicle; single service (SS) per customer versus multiple service (MS) per customer; single commodity (SC) versus multiple commodity $(\mathrm{MC})$; and hubs $(\mathrm{H})$ or transshipment points. Aircraft in the SAP usually make multiple trips. Aircraft move through the network performing multiple trips during the course of a typical planning horizon. Thus, we may add multiple trips as a characteristic of the SAP. The SAP also entails multiple services per customer (APOE) as defined by the TPFDD. Typically requirements arrive at an APOE over time and each requirement is normally much larger than a single plane load. As a result, multiple services for each APOE are required. SAP requirements are multiple commodity items that have different fill efficiencies based upon commodity type. For example, the ammunition commodity type will usually exceed the aircraft weight constraint before volume is exceeded. Conversely, the airmobile commodity type (army helicopters) normally exceeds available floor space before weight constraints. Finally, in the case where transloading is permitted, the SAP will include hubs that serve both as APOEs and APODs. In this instance, PDP precedence constraints may no longer hold. This is because the PDP assumes that the same vehicle that picks up a requirement delivers it to its ultimate destination. Transloading permits one vehicle to pickup and deliver a requirement to an intermediate point where it is subsequently picked up and delivered to its destination by at least one different vehicle. Thus, in essence, some customer nodes may be both source nodes (a pickup point) and sink nodes (a delivery point). Customers of this type are hubs (H). All of these additional characteristics with the exception of transloading 
(hubs) are included in the SAP solved in this dissertation. The additional characteristics (and the notation) are consistent with the extensions to Carlton outlined in Crino (2002). Thus, the basic SAP is classified as: MD, MV $\overline{\mathrm{H}}, \mathrm{PDP}$, RL, TW, MT, MS, MC.

There is an additional characteristic of the SAP not captured in the classification above. There are other route constraints embedded within the SAP that are a significant departure from simple route length constraints that constrain the amount of time or distance that a vehicle (aircraft) may travel. Parking MOG, working MOG, and fuel MOG constrain the timing of aircraft flow along routes. For the SAP these additional route constraints impose temporal restrictions on when aircraft may move along routes that are not specifically customer driven. For classification purposes we call these characteristics route time windows (RTW) to discriminate them from customer driven time windows.

A key feature of the SAP is that it can be broken into two components or phases: the assignment of aircraft to requirements and the subsequent routing of assigned aircraft to service missions. Unfortunately the assignment and routing components are interdependent. We need to know the routes to determine the cargo that can be loaded and we need to know the APOE-APOD assignments before the routes can be determined. This observation is pivotal in selecting a suitable SAP solution representation that captures the critical assignment and routing information while avoiding a representation that is computationally unwieldy. 


\subsection{Solution APPRoAChes to General Vehicle Routing Problems}

There is an extensive amount of work in the literature on the GVRP. Several survey papers have been done over the years such as Bodin et al. (1983), Bodin (1990), Laporte et al. (2000). The number of references in Bodin et al. alone totals 699. The following summary captures the main advances in the GVRP literature over the years and focuses on the work done on the GVRP classes that are germane to this research.

\subsubsection{Exact Algorithms}

A good survey of exact algorithms for VRPs can be found in Laporte and Nobert (1987). Exact approaches to the GVRP center around dynamic programming (DP) and Integer Programming (IP) formulations (branch and bound). Much of the initial work on the VRP focused on solving the VRP as a set-partitioning or set-covering problem. While such formulations are mathematically correct for solving the VRP, they are not practical due to the difficult and time consuming column enumeration requirement involved in solving even modest-sized problems (Bodin 1990). Balas and Padberg (1970) discuss a method of solving a set covering problem (constraints restricted to be equations or "less than or equal to" inequalities) formulated as an IP using a sequence of primal pivots instead of a cutting plane method. Balas and Padberg (1976) provide an extensive survey of the theoretical results and solution methods for the set partitioning or the equality constrained set covering problems.

Desrochers et al. (1992) formulate the VRPTW as a set covering problem. In this approach, the LP relaxation of the set covering formulation is solved using 
column generation. A branch and bound strategy is then used to find an integer solution to the set covering problem. They solve to optimality several instances of 100 customer problems. Typically, as reported in Desrochers et al., the optimal solution to the set covering problem is close to the LP relaxation solution thus making the branch and bound procedure very efficient. Bramel and Simchi-Levi (1997) demonstrate for the VRPTW why this relative gap is so small by showing that the gap becomes arbitrarily small as the number of customers grows for any distribution of service times, time windows, customer demands and customer locations.

Belenguer et al. (2000) present an interesting variant of the VRP in which the demand of a customer can be serviced by more than one vehicle. This is called the Split Delivery Vehicle Routing Problem (SDVRP). They formulate the SDVRP as an IP and solve it using a polyhedral approach. They identify some facets and develop some other valid inequalities for the SDVRP polyhedron. Unfortunately, their cutting plane algorithm was based upon a relaxed formulation of the SDVRP and only lower bounds for the SDVRP are obtained. Problem sizes solved were relatively small instances - up to 50 customers.

Further details on these exact methods can be found in Larsen (1999) and Cook and Rich (1999). Because the VRP class (and by extension the GVRP class) of problems have been shown to be NP-hard (Lenstra \& Rinooy Kan 1981), there is little hope of the existence of a polynomial time algorithm to solve them. In seeking the optimal solution, exact methods fail to meet practical time and computing resources due to problem size and complexity. As a consequence, 
exact approaches are impractical for problems of realistic size and therefore heuristic approaches are generally favored over exact methods for GVRPs.

\subsubsection{Classical Heuristics}

Classical heuristic approaches to the GVRP can be roughly separated into two categories: constructive or improvement heuristics (Laporte et al. 2000). In constructive heuristics the emphasis is on building a feasible solution without a separate improvement scheme. Improvement heuristics work on improving an incumbent solution by using some type of edge exchange heuristic within and between routes. Classical heuristics attempt to get a good solution quickly using a simple heuristic. Thus a shallower search of the solution space is characteristic of these methods when compared with metaheuristic methods.

\subsubsection{Constructive Heuristics}

Perhaps one of the most well known of the constructive heuristics for the VRP is the Clarke and Wright (1964) savings algorithm. This method attempts to construct a VRP solution by merging routes through the use of a savings criterion for a pair of customers. Laporte et al. (2000) experimented with the sequential and parallel implementations of the Clark and Wright savings algorithm and report that the parallel implementation dominates the sequential version. Gaskell (1967), Yellow (1970), Golden et al. (1977), Paessens (1988), and Nelson et al. (1985) report variations of the Clark and Wright savings algorithm which, in general, attempt to be more efficient.

Laporte et al. (2000) report on other constructive heuristics such as the petal algorithm (an extension to the sweep algorithm) first proposed by Balinski 
and Quandt (1964), the sweep algorithm attributed to Gillett and Miller (1974) and cluster-first, route-second algorithms (Fisher and Jaikumar 1981). The insertion heuristic of Solomon (1987) is a quick and effective method that is still used in constructing initial feasible tours in metaheuristics. Bramel and SimchiLevi (1995) describe a variant of the cluster-first, route-second algorithm in which the seeds used for clustering vertices are determined by solving a capacitated location problem with the remaining vertices being incorporated into routes in a second stage. Bramel and Simchi-Levi report this two-phase heuristic to be asymptotically optimal but not competitive in a practical sense. The converse, route-first, cluster-second, has also been a popular method for solving the VRP. In this method, a large (generally infeasible) route is constructed which includes all the customers. This route is then partitioned in some fashion into smaller, feasible routes. Golden et al. (1984) is an example of the route-first, cluster second method applied to the fleet size and mix VRP.

Laporte et al. (2000) performed computational comparisons of these constructive heuristics using the fourteen Christofides et al. (1979) benchmark instances for the VRP. They report good solutions using the savings algorithm of Clark and Wright (1964) and the sweep algorithms of Gillett and Miller (1974).

\subsubsection{Improvement Heuristics}

Improvement heuristics for the GVRP generally attempt to improve a particular route or set of routes through some type of edge exchange mechanism. Edge exchange mechanisms reorder some subset of customers within a route or between routes. Lin's (1965) $\lambda$-opt mechanism is typical of the edge-exchange 
methods applied to the TSP (equivalent to within route improvement for the VRP). For the $\lambda$-opt mechanism, $\lambda$ edges are removed from the tour and the $\lambda$ remaining segments are reconnected in all possible ways. Other well-known edge-exchange methods are the Or-opt (Or 1976), 2-opt* (Potvin and Rousseau 1995), 4-opt* (Renaud, Boctor and Laporte 1996) and the CROSS exchange (Taillard et al. 1997).

\subsubsection{Composite Heuristics}

Composite heuristics blend both route construction and route improvement procedures. Generally, the construction and improvement heuristics are applied in sequence when solving VRPs. Bodin (1990) reports that this approach may not work well for the more tightly constrained GVRPs. This is because the sequential nature of construction heuristics means that a bad decision in the construction phase can adversely affect subsequent steps. Improvement procedures simply may take too long or even be unable to overcome a poor decision in the construction phase. Nevertheless, composite heuristics represent a major portion of the literature on the GVRP. Examples of composite approaches for the VRPTW can be found in Derigs and Grabenbauer (1993), Kontoravdis and Bard (1995), and Russell (1995). Renaud, Boctor and Quenniche (2000) report on a composite heuristic for the PDTSP (pickup and delivery TSP) that first inserts each delivery customer simultaneously with its associated pickup customer and

then invokes an improvement procedure that uses the 4-Opt* improvement heuristic of Renaud, Boctor, \& Laporte (1996). 


\subsubsection{Constraint Programming}

Lustig and Puget (2001) and Gendreau (2002) provide excellent primers for the OR community on Constraint Programming (CP) and discuss how CP differs from traditional OR approaches. The ILOG Optimization Suite White Paper (ILOG 2001) provides an example of a comprehensive software implementation of $\mathrm{CP}$. $\mathrm{CP}$, also called constraint logic programming, is an emerging approach to solving large-scale combinatorial optimization problems such as the GVRP class. CP is the "study of computational systems based on constraints" (Bartak 1999). In CP, the idea is to solve problems by simply stating the constraints and then finding a solution that satisfies the constraints - a constraint satisfaction problem (CSP).

A CP algorithm has a two level architecture. The first level entails statements of constraints over the variables of the problem. In this level, a sort of handshaking between constraint propagation and domain reduction algorithms occur. Constrain propagation is the modification of all constraints that contain a variable whose domain has been modified. The domain reduction algorithm modifies, for each constraint, the domains of all variables in that constraint given the change in the domain of one of the variables in that constraint (Lustig and Puget 2001). A form of backtracking is used to retreat from domain reductions leading to unsatisfiable (infeasible) constraints. The handshaking between constraint propagation and variable domain reduction is carried out in an iterative

manner so as to reduce variable domains as much as possible without removing potential solutions to the CSP. The second level pertains to the methodologies 
used to program the search strategies. While the first level can determine if the CSP is infeasible, it does not necessarily find a solution if one exists. Search strategies (depth-first search, best-first search (Nilsson 1971), limited-discrepancy search (Harvey and Ginsberg 1995), depth-bounded-discrepancy search (Walsh 1997) and interleaved depth-first search (Meseguer 1997) are examples of some of the search strategies that have been implemented in commercial CP systems.

Two major positive features of $\mathrm{CP}$ are expressivity and flexibility (Gendreau 2002). Expressivity implies that complex problems are very easy to describe (express) in a natural fashion. Flexibility means that difficult problems can be solved without having to create new algorithms. Numerous and complex side constraints, typical of the GVRP class, can be easily incorporated without requiring major revisions to the algorithmic machinery. A third positive feature of $\mathrm{CP}$, called openness by Gendreau, means that $\mathrm{CP}$ can be fairly easily combined with other approaches, like local search heuristics, in a manner consistent with composite or hybrid approaches.

Gendreau (2002) cautions that CP should not be used indiscriminately. In particular, CP can be very computationally expensive when applied to welldefined (especially loosely constrained) problems (Pesant, Gendreau, and Rousseau 1997). "If one has to repeatedly solve similar instances of a problem for which other effective solution approaches exist or can be developed, I would not recommend using CP." (Gendreau 2002). In addition, mastering the technique and language of $\mathrm{CP}$ which is based on logical relationships rather than equations can be formidable. Lustig and Puget (2001) report that CP is better 
than IP in applications that concern sequencing and scheduling as well as for problems which are in essence CSPs (tightly constrained).

Current efforts in CP appear to be oriented towards combining CP with classical OR approaches and/or heuristics (hybrid approaches) in an attempt to capitalize on their respective strengths. Focacci, Lodi and Milano (2002) provide an overview of work done in combining $\mathrm{CP}$ with traditional OR methods. Examples of hybrid approaches to VRPs using CP combined with heuristics are reported in Rousseau, Gendreau and Pesant (2002), Backer et al. (2000), Caseau and Laburthe (1999) and Shaw (1998).

\subsubsection{Metaheuristics}

According to Osman and Kelly (1996):

"A metaheuristic is an iterative master process that guides and modifies the operations of subordinate heuristics to efficiently produce high quality solutions. It may manipulate a complete (or incomplete) single solution or collection of solutions at each iteration. The subordinate heuristics may be high (or low) level procedures, or a simple local search, or just a construction method."

Over the past decade, metaheuristics have been particularly popular and effective in obtaining timely and effective solutions to the GVRP class. The more popular metaheuristic approaches are simulated annealing (SA), genetic algorithms (GA) and tabu search (TS). A brief survey of the GVRP literature concerning these three approaches follows.

\subsubsection{Simulated Annealing}

SA employs the cooling or annealing of solids as a paradigm for search in combinatorial optimization problems--different states of the solid correspond to 
different solutions. Eglese (1990) provides a detailed theoretical treatment of SA. Koulmas, Antony and Jaen (1994) provide a survey of SA applications to a series of scheduling and routing problems among others. Chiang and Russell (1996) implement a SA approach to the VRPTW using the $\lambda$-interchange mechanism of Osman (1993) and a modified k-node interchange of Christofides and Beasley (1984). Osman's $\lambda$-interchange mechanism outperformed the modified k-node interchange on the larger problem instances. It can be proven that simulated annealing converges asymptotically to the optimal solution. Unfortunately, such convergence requires exponential time (Aarts and van Laarhoven 1985). Thus, in practice, simulated annealing is used with faster cooling schedules (not guaranteed to converge to the optimal) and thus it behaves like an approximation algorithm. To counter the faster cooling schedule, hybrid SA algorithms embed other methods to improve performance. Li and Lim (2001) is an example of an SA approach to the PDPTW that has an embedded TS procedure to restart the procedure after a series of non-improving iterations.

SA relies heavily on a single control parameter, the "temperature," and the cooling schedule - the rate of descent. Most importantly, SA does not make use of or exploit memory - a major drawback. For large scale problems like the SAP, knowledge gained during the course of a search can be invaluable in selecting appropriate neighborhoods and improving efficiency.

\subsubsection{Genetic Algorithms}

Goldberg (1989) and Kolen and Pesch (1994) provide good introductory information on GAs. Thangiah et al. (1991) and Thangiah (1995) introduce a 
successful GA approach to the VRPTW called GIDEON. Blanton and Wainwright (1993) introduce an implementation of a GA for the VRPTW using a set of problem specific crossover operators. A drawback of their method is that it often will not converge to a feasible solution. Moin (2002) overcomes this problem by using a hybrid GA that incorporates an insertion heuristic (Solomon 1987) and either a vertex sequencing or a parallel savings approach. Test results on a series of 30 customer problems indicate superior results for the vertex sequencing method. For a recent survey on genetic and evolutionary algorithms for the VRPTW, see Bräysy and Gendreau (2001b). GA algorithms, especially in terms of the genetic operators, can be difficult to construct.

\subsubsection{Tabu Search}

Judging by volume alone, tabu search is the most popular metaheuristic for the GVRP class. Glover (1989) introduces the fundamental ideas behind TS and Glover (1990) follows up with an analysis of refinements and more advanced aspects of TS. A foundational presentation of TS can be found in Glover and Laguna (1997) who describe TS as a "metaheuristic that guides a local heuristic search procedure to explore the solution space beyond local optimality."

Key components of basic TS are the solution $(S)$, objective function $(f(S))$, a neighborhood of the solution $(N(S))$, and a tabu memory structure. The search starts with an initial solution and defines a subset, $N(S)$, of possible solutions that are neighbors of $S$ under $N$. Each neighbor is evaluated against the objective function. In a strict local search paradigm, the best of these is selected and the process continues until it terminates at a local optimum. TS, however, employs a 
short-term memory construct to force the search beyond local optima and to prevent cycling. Attributes of recently selected moves are recorded. Moves that lead back to recently visited solutions are forbidden, or tabu. "Recently visited" is defined by the tabu tenure. Moves with tabu attributes within tabu tenure iterations are tabu. The length of the tabu tenure must be large enough to avoid cycling, but small enough not to forbid too many moves (Glover \& Laguna 1997). Given $S, N(S)$ and $f(S)$, the best non-tabu move in $N(S)$ is selected. Thus, under TS, when the search arrives at a local optimum, it does not terminate but moves beyond the local optimum by choosing the best non-tabu move. In this way, the search moves from one solution to another, with the intent of improving the quality of the solutions visited until some stopping criteria (such as maximum iterations or maximum time) is met.

There are times when excellent or influential moves (such as a move that improves the current best-known solution) may result in a tabu solution. To override such a move's tabu status, aspiration criteria are defined that may depend, for example, on the current solution and the best solution found. If the interesting move meets one of the aspiration criteria, the tabu move can be executed (Glover \& Laguna 1997).

An abundance of variations to the basic TS paradigm, described above, exist. The next section highlights some of the more important variations.

\subsubsection{Tabu Search Variations}

Other than basic TS, there are many variations of the TS methodology that have shown great promise in solving large scale combinatorial optimization 
problems. Some enhanced TS variations are vocabulary building (Kelly and Xu 1995), subpath ejection chains (Rego 1998) and path relinking (Glover, Laguna and Marti 2000). In particular, Reactive TS (RTS), Adaptive TS (ATS) and, more recently, Group Theoretic TS (GTTS) have been used successfully in a wide variety of contexts particularly routing and scheduling problems.

RTS was first proposed by Battiti and Tecchiolli (1994). RTS consists of dynamically varying the tabu tenure during the search process so as to diversify or intensify the search depending upon the current context of the search. The tabu tenure is increased if identical solutions occur too frequently and reduced when solutions have not been frequently repeated. RTS requires that a history of visited solutions be maintained and effective RTS approaches efficiently identify previously visited solutions. Typically this is achieved using some type of hashing function (see Woodruff and Zemel (1993)). GTTS provides another way to efficiently identify solutions using group theoretic constructs.

ATS changes selected tabu parameters to improve search quality by utilizing the search history. Typically the tabu tenure is perturbed dynamically to promote intensification of the search in promising regions and diversification in regions where improvements are small. If the current solution is the best solution found, the tabu tenure can be reset to the specified default value. If the current solution is better than the previous solution, but not the best solution found, the tabu tenure is decremented. If the current solution is not better than the previous solution, the tabu tenure is incremented. This dynamic updating of the tabu tenure promotes diversification and intensification (Glover \& Laguna 1997). 
There are many ATS schemes in the literature as exemplified by Chambers \& Barnes (1996), Dell'Amico \& Trubian (1993), Wiley (2001), and Combs (2002).

Group theory is one of the fundamental building blocks of abstract algebra. It is a powerful tool that has been applied to a host of disciplines such as physics, biology, cryptology and engineering (Gaglione 1992). It was also the basis for advances in exact approaches to solving integer programming problems (Gomory 1963, 1965, 1967, 1969 and Wolsey 1971a , 1971b). Until recently, however, group theory was rarely used for other problems in operations research. Colletti's (1999) landmark dissertation reveals the natural way in which combinatorial optimization problems, such as the SAP, can be couched in the unifying framework of group theory. Colletti (1999), Colletti et al. (1999), Barnes and Colletti (2000 \& 2001), Colletti and Barnes (1999a and 1999b), and Barnes, Colletti and Neuway (2002) present strong evidence of the efficacy of group theory in the study and understanding of metaheuristic approaches to combinatorial problems.

As mentioned in Section 1.4.3, there are several recent examples of the successful application of group theoretic tabu search (GTTS) to large-scale combinatorial optimization problems related to the SAP. Recent work by Wiley (2001) on the Aerial Flight Refueling Problem (AFRP), Crino (2002) on the Theater Distribution Vehicle Routing and Scheduling Problem (TDVRSP) and Combs (2002) on the Crew Scheduling Problem clearly demonstrate that GTTS can provide robust, effective and efficient solutions to practical large scale combinatorial optimization problems. 


\subsubsection{Tabu Search Applications to the GVRP}

TS applications to the GVRP and its variants are voluminous and a full survey is beyond the scope of this review. Instead key contributions in the literature are highlighted with emphasis on those applications that possess aspects of the SAP.

Gendreau et al (1999) introduced a TS heuristic developed for the heterogeneous fleet vehicle routing problem. This is an extension of the classical VRP that includes a heterogeneous fleet with various vehicle capacities. The quite elaborate heuristic incorporates a generalized insertion heuristic, a route optimizer and TS embedded within an adaptive memory procedure. Gendreau et al (1999) employ Gendreau and Laporte's (1994) dynamic diversification mechanism that penalizes vehicle capacity constraints violations. This penalty structure is typical of TS applications and permits traversal of infeasible regions while encouraging a return to feasibility. Toth and Vigo (2003) introduce Granular TS (GTS) and its application to the capacitated and distance constrained VRP. GTS is based on significantly reduced neighborhoods called granular neighborhoods obtained by ignoring neighboring solutions with attributes unlikely to belong to high quality solutions. Granular neighborhoods can be viewed as a variant of the candidate list strategies described in Glover and Laguna (1997) and are best employed with problem structures whose solution cost is the sum of the included elements.

TS implementations for the VRPTW are numerous as well. Rochat and Semet (1994) develop a TS approach to a VRPTW that has many elements of the 
SAP not usually captured in the VRP literature. Some examples are vehicle accessibility issues (akin to airfield constraints), route time duration (crew constraints), and differing vehicle capacities. In Carlton (1995) and Barnes and Carlton (1995), a robust RTS approach to the VRPTW is implemented using simple insertion moves to define the neighborhood. Hashing structures, critical to efficiency in RTS, are also described. Potvin et al. (1996) implement a TS approach to the VRPTW that maintains feasibility throughout. They incorporate a dynamic neighborhood that alternates between 2-opt* and Or-opt neighborhoods to diversify the search. Interestingly, they conclude that better results may be obtained by relaxing vehicle capacity constraints thus allowing traversal of infeasible solutions. Taillard et al. (1997) present a TS approach to the VRPTW in which the time windows are considered to be soft. They introduce a new edge exchange heuristic called CROSS exchange that generalizes two edge exchange heuristics such as 2-opt* (Potvin and Rousseau, 1995) and Or-opt (Or 1976). A key feature of CROSS exchange is that it preserves the orientation of the routesa useful feature for problems with time windows. A drawback of the CROSS exchange is its complexity and therefore approximations are used to evaluate moves in constant time. Tests on Solomon's set of VRPTW (hard time windows) resulted in 17 improvements and 20 ties to the best known solution on the 56 test problems. Chiang and Russell (1997) develop a RTS in which the underlying local search is based on the $\lambda$-interchange mechanism of Osman (1993). More recent TS implementations for the VRPTW are Gehring and Homberger (1999 and 2001), Tan et al. (2000), and Cordeau et al. (2001). 
TS implementations have also been applied to the more difficult PDPTW class of problems that are generalizations of the VRPTW. Nanry (1998) and Nanry and Barnes (2000) extend the work of Carlton (1995) on the VRPTW with a RTS approach to solving the PDPTW. Solomon's (1987) insertion method is used to construct a feasible PDPTW solution. An RTS method is then invoked to improve the plan using three neighborhoods, namely, Single paired insertion (SPI), Swapping pairs between routes (SBR) and Within route insertion (WRI). Lau and Liang (2001) implement a RTS approach to the PDPTW that incorporates a unique construction heuristic to build a feasible initial solution. Their partitioned insertion heuristic combines an insertion heuristic with a sweep heuristic. The advantage of including the sweep heuristic is that near and far customers are included in the same route that has a balancing effect across routes. The neighborhood moves are those of Nanry and Barnes (2000).

\subsubsection{Adaptive Tabu Search Approach to the SAP}

There are many approximation methods used to solve vehicle routing and scheduling problems and their variants (GVRP). This literature review indicates that the best of these is tabu search which has been used extensively over the past decade to solve GVRPs. In recent years, TS applications have provided the best solutions in the least amount of time for many instances of the GVRP. Because of this strong track record, a TS approach to the SAP was deemed appropriate for this research.

Among the major variants of TS, which would be most appropriate? Both GTTS and RTS approaches were investigated as potential TS methodologies to 
employ for the SAP. Due to the complex SAP problem structure, however, neither was deemed appropriate and therefore an ATS approach was adopted.

As noted above, GTTS has proven very effective with such problems as VRPs and CSPs - partitioning and ordering problems $(\mathrm{P} \mid \mathrm{O})$. A GTTS approach entails formatting the representation of the SAP using the Symmetric Group on $n$ letters (see Crino (2002) for a detailed description). For a VRP, letters represent vehicles and customers. Letters must be unique therefore multiple letters are needed for customers requiring multiple services. Formatting the SAP using the symmetric group on $n$-letters is possible but inefficient. Unique letters would be needed to capture not only aircraft and customer information, but also the more complex routing information in the SAP. Since routes are used repeatedly by different aircraft, unique letters would need to be used to capture each use. Even for moderately sized problems, the number of letters required would be prohibitive. Routing also precludes the SAP from being cast completely as a $\mathrm{P} \mid \mathrm{O}$ problem. For the SAP, the load an aircraft can carry is not just a function of aircraft (capacity) and customer (requirement). An allowable load in the SAP is also a function of the route selected. The assignment of aircraft to requirements by itself can be cast as a $\mathrm{P} \mid \mathrm{O}$ problem, but the routing of aircraft cannot.

For a RTS approach to be effective, a complete accounting of the solutions visited during the search must be maintained. This is difficult to achieve efficiently in the SAP. The SAP solution representation must capture the routing of each trip in each aircraft's mission. Two solutions that contain a mission that passes through the same routes may not be equivalent due to different timings and 
loads. For practical sized SAP instances, any solution hashing scheme quickly becomes unwieldy and inefficient.

For the above reasons, an ATS approach to the SAP was developed. An ATS approach still employs an adaptive procedure to intensify and diversify the search based upon the search history without requiring as detailed an accounting. ATS exploits the key facets of TS and, as documented in this dissertation, provides timely solutions to decision makers at AMC that are superior to those rendered by competing techniques.

This chapter has provided a review of the literature associated with the SAP. Chapter 3 provides a detailed problem description of the SAP and the solution representation used. 


\section{Chapter 3: $\quad$ Detailed Problem Statement}

This chapter provides a detailed description of the SAP and the solution representation used in this research.

\subsection{The Strategic Airlift Problem}

A stated objective of this research is to develop a decision support tool that provides excellent solutions to the SAP in reasonable time with the level of detail compatible with AMOS version 2.0. Incorporation of such a decision support system into AMOS should greatly improve the performance of AMOS.

Decision support tools such as AFM and AMOS are the result of extensive experience and insights gained over time at AMC. Wherever possible, this knowledge was incorporated to properly characterize the framework for the SAP.

\subsubsection{SAP Inputs}

The airlift network described in Chapter 1 forms the basic framework for the SAP. The following discussion details each of the components of the airlift network that are provided as input to the model. AMOS builds these files based upon information contained in the TPFDD and user input. Their main components are requirements, aircraft, locations and routes.

\subsubsection{Requirements}

Requirements are contained in the AMOS file reqts.dat. Data within this file is extracted from the TPFDD. The key attributes of each requirement are:

- Type and quantity of cargo to be delivered (outsize, oversize, and/or bulk)

- Commodity type for the requirement (Navy_Marines, CSS_Other, ...) 
- Number of personnel to be moved

- Available to Load Date (ALD)

- Required Delivery Date (RDD)

- APOE and APOD locations

- Priority

While a requirement can consist of any combination of cargo types or personnel, each requirement has a unique commodity type. The commodity type is derived from the requirement's unit type which, in turn, determines the fill efficiency achievable for a given aircraft. Fill efficiencies or payload targets are defined in the AMOS file payloadtgt.dat. A non-exhaustive listing of the payload targets for a C-5A aircraft is given in Figure 3.1.

\begin{tabular}{|c|c|c|c|c|c|c|c|c|}
\hline $\begin{array}{ll}/ / & \text { Type } \\
/ / & \text { Commodity }\end{array}$ & Oüt $>0$ & Over $>0$ & Bulk $>0$ & $\begin{array}{l}\text { Over }>0 \\
\text { Out }=0\end{array}$ & $\begin{array}{l}\text { Bulk } \\
\text { Out }=0\end{array}$ & $\begin{array}{l}\text { Bulk } \\
\text { Both=0 }\end{array}$ & $\begin{array}{l}\text { Pax } \\
\text { Fill }\end{array}$ & $\begin{array}{l}\text { Pax } \\
\text { Only }\end{array}$ \\
\hline \multicolumn{9}{|c|}{ // -------------------------------------------------------------------------------1} \\
\hline "AF_Acft" & 41.3 & 20.6 & 0.0 & 51.1 & 1.2 & 68.4 & 71 & 340 \\
\hline "AF_Supt" & 42.6 & 17.0 & 0.0 & 64.6 & 0.2 & 68.4 & 71 & 340 \\
\hline "туре_3" & 34.5 & 38.9 & 0.0 & 72.6 & 0.0 & 82.8 & 71 & 340 \\
\hline "Type_4" & 34.5 & 38.9 & 0.0 & 72.6 & 0.0 & 82.8 & 71 & 340 \\
\hline "Army_Prepo" & 34.5 & 38.9 & 0.0 & 72.6 & 0.0 & 82.8 & 71 & 340 \\
\hline "Airborne" & 18.0 & 13.4 & 0.2 & 52.0 & 5.7 & 82.8 & 71 & 340 \\
\hline "Armor" & 61.5 & 20.5 & 0.0 & 79.4 & 0.0 & 82.8 & 71 & 340 \\
\hline "Mech" & 65.3 & 16.3 & 0.0 & 80.3 & 0.0 & 82.8 & 71 & 340 \\
\hline "Airmobile" & 15.5 & 11.9 & 0.1 & 51.5 & 7.7 & 82.8 & 71 & 340 \\
\hline "Cbac" & 18.5 & 10.0 & 1.1 & 53.2 & 9.5 & 82.8 & 71 & 340 \\
\hline "Infantry" & 34.1 & 16.0 & 0.1 & 55.6 & 6.6 & 82.8 & 71 & 340 \\
\hline "Armor_Cav" & 58.1 & 16.8 & 0.0 & 63.1 & 12.5 & 82.8 & 71 & 340 \\
\hline "Type $\overline{1} 3 "$ & 0.0 & 0.0 & 0.0 & 0.0 & 0.0 & 78.3 & 71 & 340 \\
\hline "Navy_Marines" & 29.0 & 13.9 & 0.0 & 62.4 & 0.1 & 78.3 & 71 & 340 \\
\hline "CS_Eng" & 54.4 & 18.3 & 0.2 & 65.2 & 10.1 & 82.8 & 71 & 340 \\
\hline "CS_Artillery" & 65.1 & 13.7 & 0.0 & 63.2 & 10.7 & 82.8 & 71 & 340 \\
\hline "CS-Other" & 57.5 & 14.7 & 1.4 & 66.0 & 7.8 & 82.8 & 71 & 340 \\
\hline 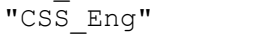 & 54.0 & 13.6 & 0.4 & 66.9 & 9.8 & 82.8 & 71 & 340 \\
\hline "CSS-Med" & 37.9 & 20.5 & 0.0 & 56.6 & 8.2 & 82.8 & 71 & 340 \\
\hline "CSS_Signal" & 46.1 & 19.0 & 0.4 & 54.8 & 6.4 & 82.8 & 71 & 340 \\
\hline "CSS_Supt_maint" & $=" 52.1$ & 17.3 & 0.4 & 62.1 & 9.0 & 82.8 & 71 & 340 \\
\hline "CSS-Trans" & 61.7 & 14.1 & 0.1 & 70.3 & 3.3 & 82.8 & 71 & 340 \\
\hline "CSS_other" & 34.5 & 16.9 & 1.4 & 66.2 & 5.9 & 82.8 & 71 & 340 \\
\hline "Typē_24" & 34.5 & 17.9 & 0.4 & 62.1 & 9.0 & 78.3 & 71 & 340 \\
\hline "Resupply" & 34.5 & 16.7 & 1.6 & 64.0 & 7.6 & 82.8 & 71 & 340 \\
\hline "Ammo" & 0.0 & 0.0 & 0.0 & 0.0 & 0.0 & 90.0 & 0 & 340 \\
\hline
\end{tabular}

Figure 3.1 Extract from AMOS Payload Target File for C-5A 
Each number gives the maximum load (tons or number of personnel) for the associated commodity. The first three columns are related to available outsize cargo; columns four and five are used if no outsize cargo is available and oversize cargo is available; column six is used if only bulk is available; column seven gives the number of seats available for personnel if some cargo is loaded and column eight is the number of seats available if only personnel are loaded. Additional details regarding how payload targets are used are described below.

The requirements in the reqts.dat file are ordered according to ALD, RDD and priority. The ALD and RDD define the time window for the requirement. Aircraft may arrive at an APOE prior to the ALD, but, in general, MOG restrictions make it preferable to arrive "just in time." A requirement is deemed on time if all of the requirement arrives on or prior to its RDD. Finally, smaller integer requirement priority values indicate a higher priority when compared to other requirements. Priority is used to discern between requirements with identical ALD and RDD windows.

\subsubsection{Aircraft}

Several files define aircraft attributes and availability for an instance of the SAP. The AMOS file typeac.dat defines the key characteristics of each type aircraft available. These attributes include the following:

- Type aircraft $(\mathrm{C} 5-\mathrm{A}, \mathrm{C}-17, \ldots)$

- Cruising speed

- Cruising altitude

- Fuel configuration (capacity, holding fuel, approach \& landing fuel, taxi to takeoff fuel, minimum landing fuel)

- Allowable cabin load (ACL)

- Trivial load 
- Standard times (ground times, onload time, offload time)

- Cargo compatibility (Preferred, Feasible, Incompatible)

- Body Type (Wide, Narrow, or Small)

An aircraft's ACL is the maximum weight allowed for a given payload. The trivial load represents a threshold weight below which, ideally, cargo would not be carried. In this implementation all requirements are delivered even if delivery results in a mission with a trivial load. The cargo compatibility defines an aircraft's compatibility with outsize, oversize, and bulk cargo as well as personnel. Compatibility is partitioned into three categories: preferred, feasible, and incompatible. An aircraft's body type is used in determining the parking and working MOG assets an aircraft requires.

Fuel consumption rates (climb and cruise) for each type aircraft are defined in the AMOS file fuelcalc.dat. The AMOS file airunit.dat defines the aircraft available for a given instance. This file defines each available aircraft's type, home base, and time available for the aircraft in a given SAP instance. Also defined is the maximum continuous number of hours an aircraft may remain away from its home base- the return to base time (RTB). After this time limit, no further missions may be scheduled until the aircraft has returned to its home base and undergone maintenance. Thus, missions that are completed after the limit can be scheduled as long as the mission is commenced prior to the return to base limit. Once this mission is complete the aircraft must return to base. Finally, the uterate.dat file defines the utilization (UTE) target rate by aircraft type and stipulates the total, daily airlift capability for a particular fleet of aircraft, i.e., the 
UTE rate is the total pool of daily flying hour capability for a fleet of aircraft distributed equally among each aircraft.

\subsubsection{Locations}

The locations that make up the airlift system are defined in three AMOS files: airbase.dat, recover.dat, and waypoint.dat. All locations are assigned an identifier. The identifier for airbases and recovery bases are usually the fourletter International Civil Aviation Organization (ICAO) identifier. Waypoints are assigned alphanumeric identifiers by AMC. The airbase file defines the bases in the airlift system for a particular instance. Key attributes of airbases include:

- Latitude/Longitude

- Region

- Maximum on ground (MOG)

$\circ$ Working MOG (Wide \& Narrow)

○ Parking MOG (Wide \& Narrow)

- Daily fuel capacity

- Alternate landing base location (Civilian \& Military)

- Compatible aircraft (Military or Civilian; Wide or Narrow)

- Permissions (operating hours, etc)

Airbases may be home bases for aircraft units, APOEs, APODs, en route bases, or recovery bases. Some bases may satisfy more than one category. For example, a home base may also be an APOE and serve as an en route base for one or more routes. Home bases are specified in the airunit.dat file. APOEs and APODs are designated in the reqts.dat file. En route bases are specified in the plan.dat file and recovery bases are defined in the recover.dat file. These will be described in more detail below. 
Each airbase is assigned to a region. Generally, several bases will share a region. This clustering of bases into regions reduces the number of possible routes in the airlift network by defining routes to connect regions rather than individual bases. Routing in the SAP will be discussed in more detail below. Figure 3.2 below shows the regions defined by AMC and used in constructing routes.

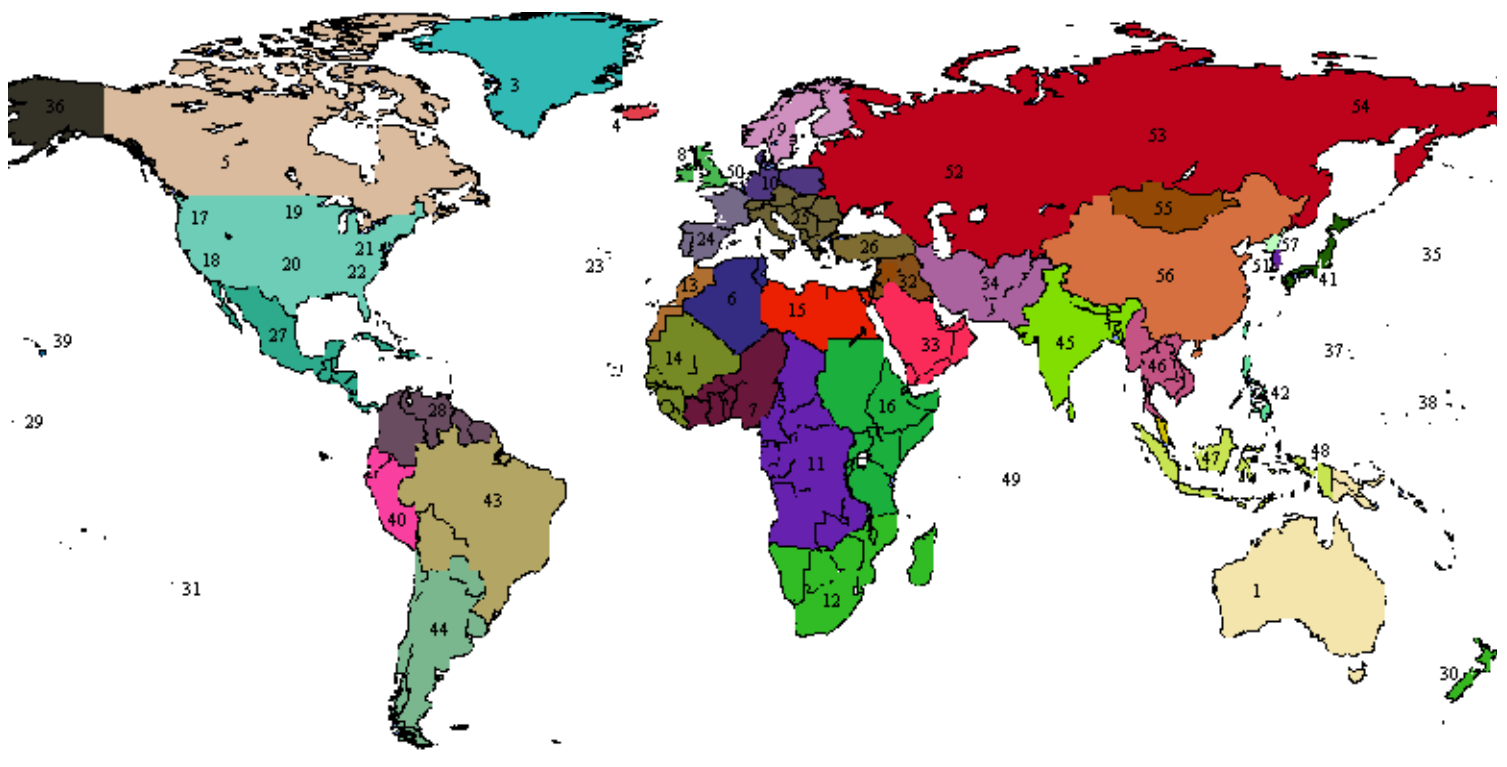

Figure 3.2 Region Map

Parking and working MOG at an airbase are an aggregation of the capacity of an airfield to park and service various aircraft at any given time. Daily fuel capacity is the number of gallons of fuel that an airbase apportions to airlift on a daily basis. Alternate airfields are where an aircraft is diverted if congestion or other circumstances preclude landing at the primary airbase. In this research, 
alternate airfields are used only in determining fuel requirements for a particular mission. A portion of the fuel requirements for a mission must include enough fuel to fly the aircraft to an alternate airbase.

Compatible aircraft for an airbase are partially defined by the wide-body and narrow-body MOG values - if the wide-body working MOG is zero, widebody aircraft are not allowed at that airbase. More detail on compatible aircraft for an airbase are described in the permission.dat file which defines what activities can take place at an airbase based upon the type of aircraft. These activities include whether it can be used as an en route airbase, whether refueling is available, and whether onloading and/or offloading can occur. These permissions can change over time.

Recovery bases are defined in the AMOS recover.dat file. This file defines where an aircraft will recover once it has offloaded at an APOD based upon the APOD region and the type of aircraft. If no recovery base is specified in this file, then the aircraft will recover at its APOD.

The last type of location is the waypoint - a point in space used as a routing control measure or as a rendezvous for aerial refueling. Waypoints are specified in the file waypt.dat in which the waypoint's location (latitude/longitude) and region are defined.

\subsubsection{Routes}

All possible routes for a SAP instance are defined in the AMOS file plan.dat which is the most complicated of the AMOS input files. AMC planners build the plan.dat file based upon experience and the current situation. The first 
of three parts defines the various aircraft groups. These groups are used to define specified routes based upon aircraft properties such as whether the aircraft is military or civilian or of a specific type such as C-5As. The second part of the plan.dat file, the planning group list, defines all the currently planned routes. Each planning group has a name, a source region, a sink region and an aircraft group. As an example, a plan which links source region 20 to sink region 33 for the aircraft group "C-5s" would be named "20_33_C-5S_1" where the one represents the fact that this is first such plan for the given source, sink and group.

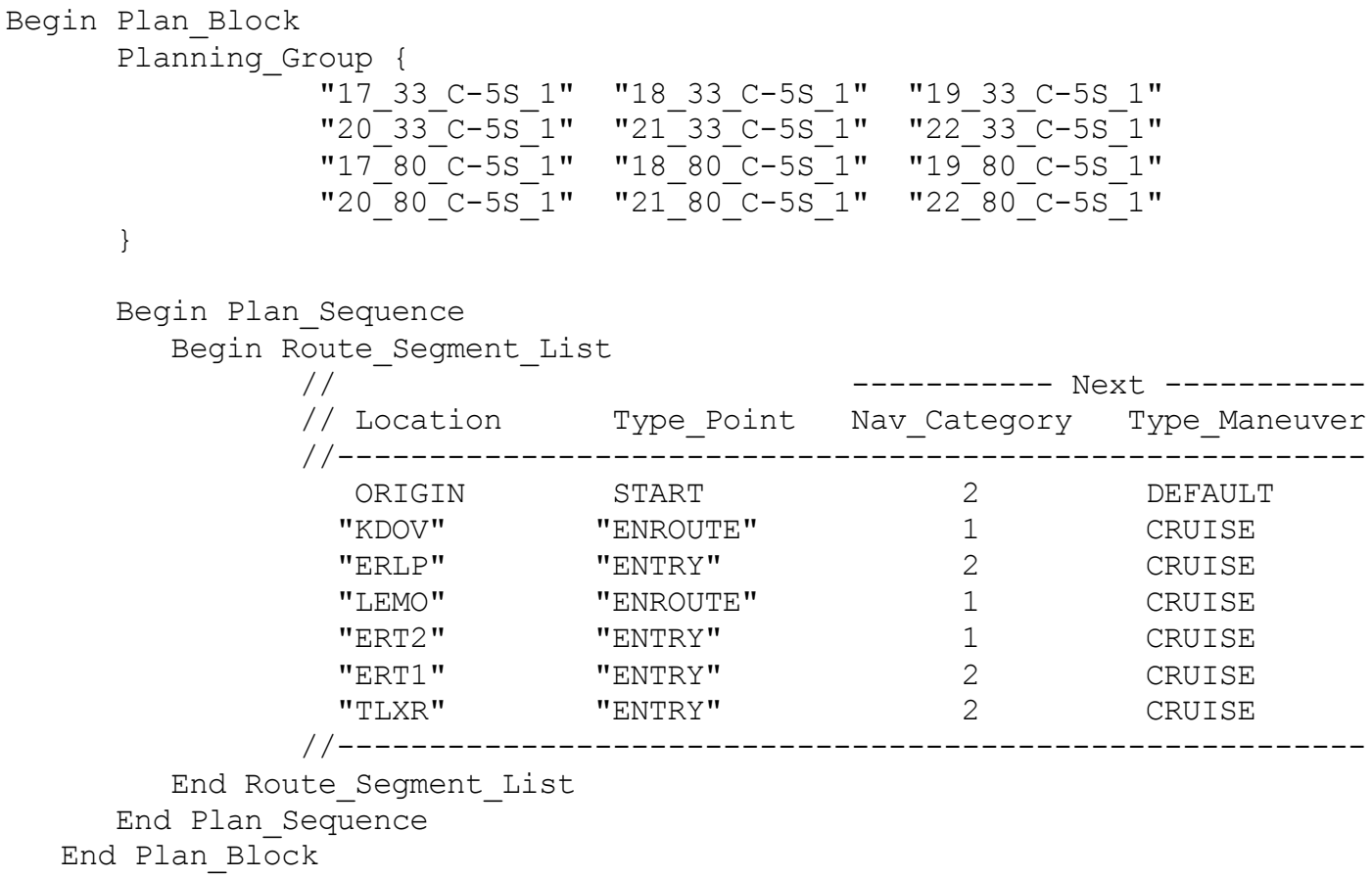

Figure 3.3 Route Segment Extract from AMOS Plan File

The third and final part of the plan document defines the route segments that join the source and sink regions for the various planning groups. An extract from the 
third part of the AMOS plan file for the route segment above is shown in Figure 3.3.

Note that several planning groups may share the same route segment. This is how the reduction in number of possible routes in modeling the airlift system is realized. Essentially bases are clustered in regions and regions are joined together by route segments. Continuing the example, the planning group "20_33_C-5S_1" connects region 20 to region 33 according to the route segment in Figure 3.3. The aircraft would depart from its origin base in region 20 and fly to KDOV (KDOV is the ICAO symbol for Dover Airbase in Delaware) where it would land and undergo en route base activities such as refueling and routine maintenance. From KDOV it would fly via the waypoint ERLP (entry signifies a waypoint) to LEMO (Moron Airbase in Spain) where it would once again land and undergo en route base activities. From LEMO, the aircraft would pass in succession through the waypoints ERT2, ERT1 and TLXR and then finally land at its destination base in region 33. A route is the path defined by an origin, route segment and destination. The only differences between the routes "20_33_C5S_1" and "22_80_C-5S_1" are the starting and ending bases (all intermediate stops are identical).

The navigation categories (NAVCAT) define whether additional fuel reserves are required due to flight over water. Category 1 requires a minimum of $10 \%$ and one hour of cruise fuel be added to fuel requirements. Category 2 requires no additional fuel reserves. Each plane's return trip is defined by a 
different planning group and route segment that may or may not be the reverse of the outbound trip.

\subsubsection{Additional SAP Characteristics}

There are several characteristics of the SAP that are critical to understanding later discussions. The next several sections describe: standard flight legs, how aircraft payloads are determined, how maximum cargo for a route is determined, how mission timings are determined, and how fuel requirements are calculated.

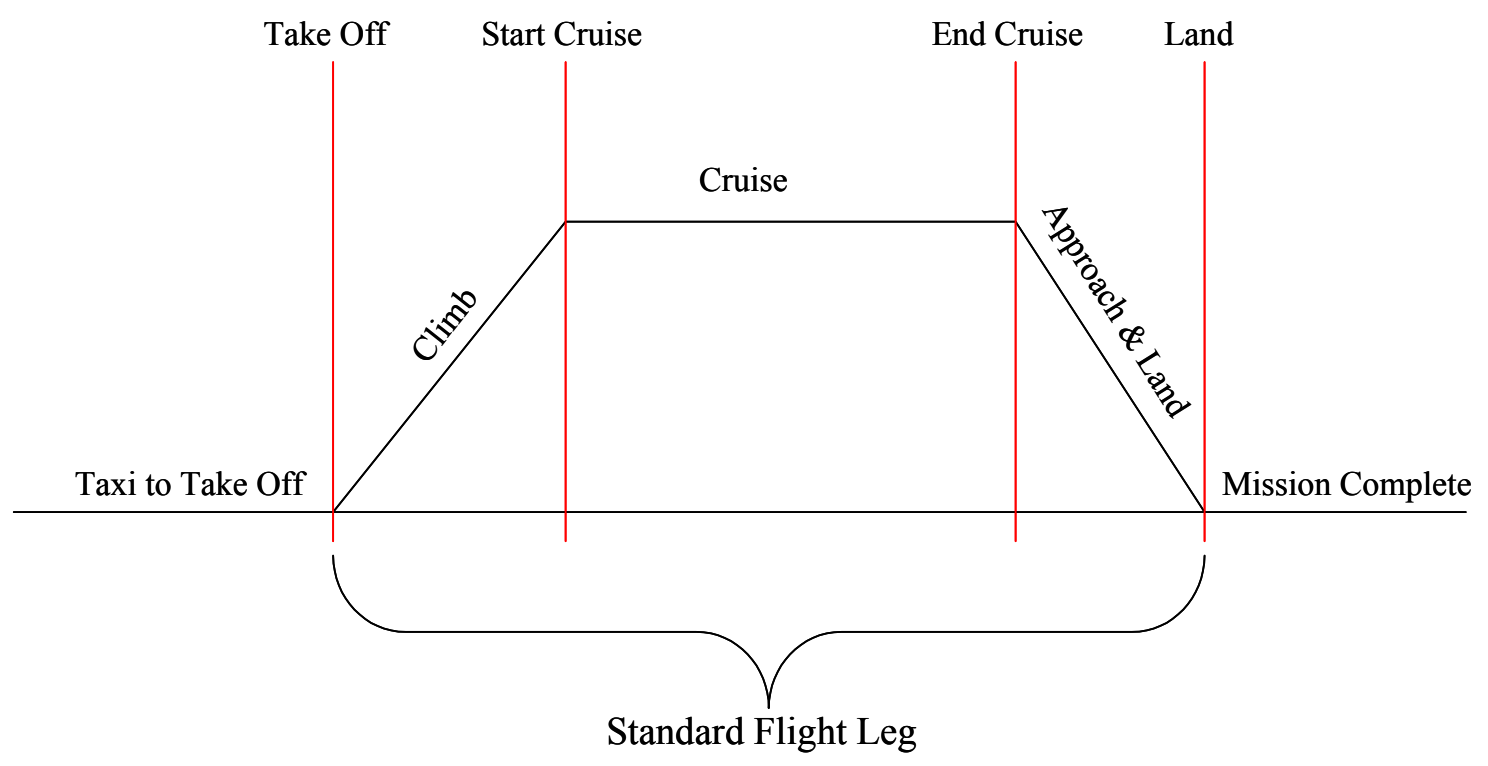

Figure 3.4 Standard Flight Leg with Segments

\subsubsection{A Standard Flight Leg}

A flight leg consists of several segments: climb, cruise, approach, landing and alternate base. The alternate base segment helps determine fuel requirements 
in the event an aircraft must be diverted from its original destination. A graphical depiction of a flight leg is shown in Figure 3.4.

The flight leg is the basis for calculating maximum cargo for an APOE to APOD route, determining mission timings, and calculating fuel requirements.

\subsubsection{Calculating Maximum Cargo for a Route}

For each APOE-APOD route and aircraft type, there is a maximum payload weight that can be carried based upon the route critical leg and the aircraft characteristics. The route critical leg is the flight leg with the smallest maximum payload. This is calculated independent of volume issues associated with commodity and cargo types. Issues with fill efficiencies are considered during the loading process using the payload target data.

Given the critical leg, distance to the alternate base, and aircraft type, the maximum payload weight for a given route and aircraft type is computed. In the first of two steps, the aircraft is hypothetically filled to its maximum fuel capacity and the maximum cargo possible is hypothetically loaded (subject to ACL or maximum take-off weight restrictions). Assuming this configuration, fuel consumption for the critical leg is calculated. In the second step, if unused fuel remains, its weight difference is added to the maximum cargo value. If the projected fuel consumed exceeds the fuel capacity, the maximum cargo is reduced by the weight of the excess consumed fuel. Next, an iterative non-linear binary search is performed to determine the projected maximum cargo because the reduction in payload-fuel consumption trade-off is not linear. This procedure is used to determine the maximum cargo for each route and aircraft type. 
As an example maximum weight calculation, assume that a $\mathrm{C}-5 \mathrm{~A}$ is carrying cargo from KGRK to OKBK and is using the route segment of Figure 3.3. The aircraft speed is 0.74 mach and flight level is 31,000 feet. The key features of the route are shown in Table 3.1. Airbases and waypoints are named according to the ICAO abbreviations.

\begin{tabular}{|l|l|l|l|l|l|c|}
\hline FROM & TO & TYPE POINT & DIST & $\begin{array}{l}\text { ALT } \\
\text { BASE }\end{array}$ & $\begin{array}{l}\text { DIST } \\
\text { TO } \\
\text { ALT }\end{array}$ & $\begin{array}{l}\text { NAV } \\
\text { CAT }\end{array}$ \\
\hline KGRK & KDOV & EN ROUTE & 1196 & KWRI & 67 & 2 \\
\hline KDOV & ERLP & ENTRY & 2229 & NONE & 0 & 1 \\
\hline ERLP & LEMO & EN ROUTE & 1018 & LERT & 48 & 2 \\
\hline LEMO & ERT2 & ENTRY & 698 & NONE & 0 & 1 \\
\hline ERT2 & ERT1 & ENTRY & 1232 & NONE & 0 & 1 \\
\hline ERT1 & TLXR & ENTRY & 264 & NONE & 0 & 2 \\
\hline TLXR & OKBK & DESTINATION & 836 & OKAJ & 21 & 2 \\
\hline
\end{tabular}

Table 3.1 Route Segment Details for KGRK - OKBK Route

Distances are measured in nautical miles and calculated using the great circle distance method. The great circle distance method takes into account the curvature of the earth in calculating the distance between two points. Given the latitude and longitude of two points on the surface of the earth, the shortest distance between the two points is given by the length of an arc of a circle 
concentric with the earth and with the same radius as the earth. The great circle distance is calculated as follows:

distance $=60 * \arccos \left(\begin{array}{l}\sin (\text { lat } 1 * \pi / 180) * \sin (\text { lat } 2 * \pi / 180)+\cos (\text { lat } 1 * \pi / 180) \\ * \cos (\text { lat } 2 * \pi / 180) * \cos ((\text { lon } 2-\text { lon } 1) * \pi / 180)\end{array}\right) * 180 / \pi$

where lat1 and lat2 are the latitudes and lon 1 and lon 2 are the longitudes for the first and second points respectively.

In Table 3.1, the critical leg passes through KDOV-ERLP-LEMO for a distance of 3,247 nautical miles. Table 3.2 details the required flight characteristics of the C-5A. These values are contained in the AMOS file typeac.dat.

\begin{tabular}{|l|l|l|l|l|l|}
\hline $\begin{array}{l}\text { Weights } \\
\text { (lbs) }\end{array}$ & ACL & Max Takeoff & Max In Flight & Empty & Trivial Load \\
\hline Value & 185,822 & 769,000 & 769,000 & 372,500 & 52,000 \\
\hline Fuel (lbs) & Capacity & Holding & Approach \& Land & Taxi to Takeoff & Min Landing \\
\hline Value & 332,500 & 18,000 & 5,200 & 3,000 & 0 \\
\hline
\end{tabular}

Table 3.2 C-5A Standard Weights and Fuel

The fuelcalc.dat file contains the climb and cruise data for all aircraft types. Figure 3.5 is an extract of the climb data section for a C-5A which provides the quantity of fuel burned (in pounds), distance traveled during climb (nautical miles), and amount of time to reach altitude (minutes) based upon the type aircraft, its weight and cruising flight level (in feet). Figure 3.6 is an extract 


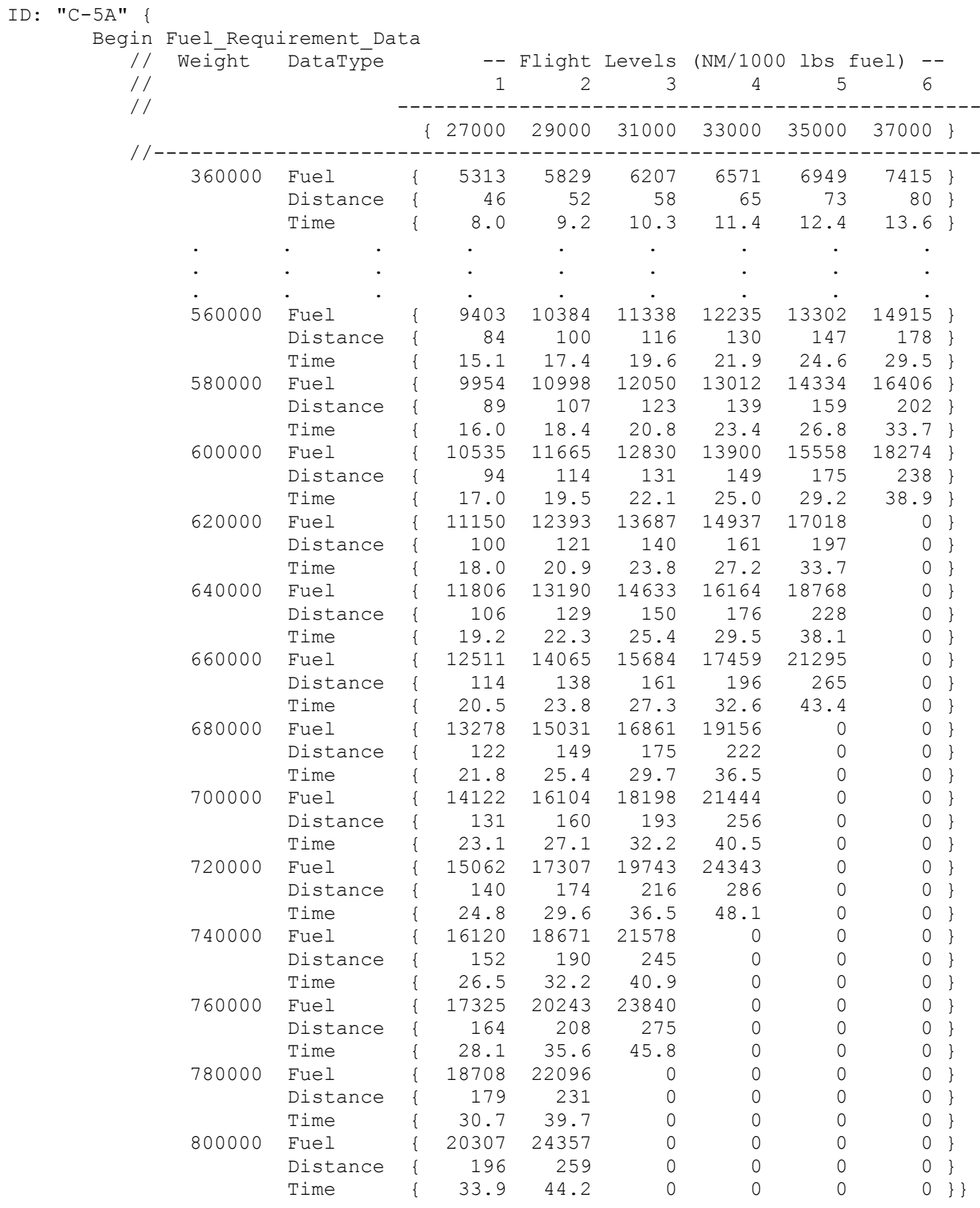

Figure 3.5 Climb Data Extract from fuelcalc.dat File for a C-5A 
of the cruise segment data from the fuelcalc.dat file. The cruise section provides the burn rate (expressed in nautical miles per 1,000 pounds of fuel) for a given aircraft type, cruising speed, weight and flight level.

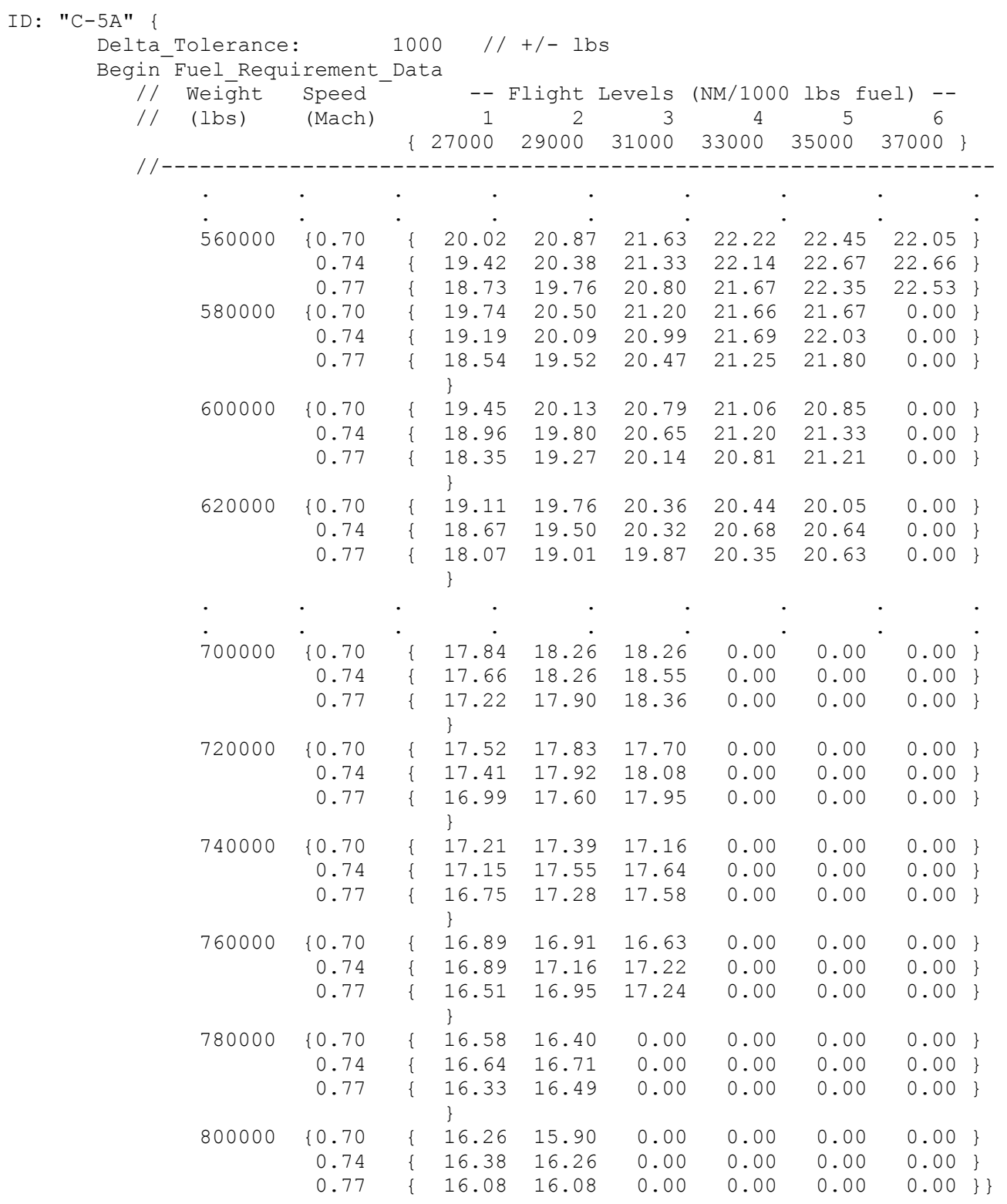

Figure 3.6 Cruise Data Extract from fuelcalc.dat file for a C-5A 
Using the computation procedure described above, the maximum weight is determined as follows:

- Find the maximum weight for the aircraft with full fuel tanks:

$$
\begin{aligned}
\mathrm{acWgt}=\mathrm{emptyWgt}+\text { fuelCapWgt }=372,500+332,500=705,000 \\
\begin{aligned}
\operatorname{maxCargo} & =\min (\text { MaxTakeOff, maxTableWgt })-\mathrm{acWgt} \\
& =\min (769,000 ; 760,000)-705,000=55,000
\end{aligned}
\end{aligned}
$$

The maxTableWgt is determined from the fuelcalc.dat file using the maximum feasible climb weight $(760,000$ in this case). (Zero entries in the climb and cruise tables indicate infeasible combinations.)

$$
\mathrm{acWgt}=\mathrm{acWgt}+\operatorname{maxCargo}=705,000+55,000=760,000
$$

- Determine the climb segment values

$$
\text { climbWgt }=\text { acWgt }- \text { taxiToTakeOff }=760,000-3,000=757,000
$$

Since an aircraft weight of 757,000 is not one of the weight levels in the climb data table (Figure 3.5), linear interpolation is performed using the weight levels that bracket the climbWgt $(740,000 \& 760,000)$. The fuel burned during the climb is 23,500 ; the distance traveled is 271 nautical miles.

- Determine average cruise burn rate

The average cruise burn rate is computed as the average of the burn rate at the start of the cruise segment and at the end of the cruise segment.

$$
\text { startCruiseWgt }=\text { climbWgt }- \text { climbFuel }=757,000-23,500=733,500
$$

The interpolated value for start cruise burn rate is $17.78 \mathrm{~nm} / 1000 \mathrm{lbs}$ of fuel.

$$
\text { cruiseDist }=\text { legDist }- \text { climbDist }=3247-271=2976
$$


cruiseFuel $=(1 /$ rate $) *$ cruiseDist $* 1000$

$=(1 / 17.78) * 2976 * 1000=167,379$

endCruiseWgt $=$ startCruiseWgt - cruiseFuel

$$
=733,500-167,379=566,121
$$

The interpolated value for the end of cruise burn rate is $21.23 \mathrm{~nm} / 1000 \mathrm{lbs}$ of fuel.

avgCruiseBurnRate $=(17.78+21.23) / 2=19.51$

- The acWgt at end of cruise, using the average burn rate, is recalculated as

cruiseFuel $=(1 /$ rate $) *$ cruiseDist $* 1000$

$=(1 / 19.51) * 2976 * 1000=152,606$

endCruiseWgt $=$ startCruiseWgt - cruiseFuel

$$
=733,500-152,606=580,894
$$

- Calculation of the alternate base fuel requirement

The burn rate to the alternate base is calculated using the endCruiseWgt.

The interpolated value is $20.98 \mathrm{~nm} / 1000 \mathrm{lbs}$ of fuel.

altFuel $=(1 /$ rate $) *$ altDist $* 1000=(1 / 20.98) * 48 * 1000=2288$

- Calculation of the required fuel reserves

Required reserves include the alternate base fuel, minimum landing fuel, holding fuel, and, if applicable, a NAVCAT 1 reserve. The standard reserve is calculated as follows:

$$
\begin{aligned}
\text { stdReserve } & =\max (\text { minimum landing, holding }+ \text { altFuel }) \\
& =\max (0,18000+2288)=20,288
\end{aligned}
$$

If part of the cruise segment is navigation category 1 , an additional reserve must be included. From Table 3.1, the flight from KDOV to ERLP is a navigation category 1 flight. The required NAVCAT 1 reserve is equal to the 
minimum of $10 \%$ of the fuel for this portion of the cruise segment and one hour of cruise fuel. To compute one hour of cruise fuel, the distance traveled in one hour at the given speed is determined, and the avgCruiseBurnRate is used.

$$
\begin{aligned}
\text { NAVCAT1Fuel } & =\min (10 \% * \text { cruiseFuel, } 1 \text { hour cruise fuel }) \\
& =\min (10 \% * \text { cruiseFuel, }(1 / \text { rate }) * \text { oneHourDist*1000 }) \\
& =\min (10 \% * 100413,(1 / 19.51) * 437 * 1000) \\
& =\min (10041,22399)=10041
\end{aligned}
$$

- Calculation of the total fuel required

$$
\begin{aligned}
\text { totFuelBurned } & =\text { taxiToTakeOff }+ \text { climbFuel }+ \text { cruiseFuel }+ \text { appLandFuel } \\
& =3000+23,500+152,606+5200=184,306 \\
\text { totFuelRequired } & =\text { totFuelBurned }+ \text { requiredFuelReserves } \\
& =184,306+30,329=214,635
\end{aligned}
$$

- Determination of whether there is surplus fuel at landing

$$
\begin{aligned}
\text { extraFuel } & =\text { fuelCapWgt }- \text { totFuelRequired } \\
& =332,500-214,635=117,865
\end{aligned}
$$

Since the extraFuel value is positive, the excess is added to the current maxCargo to obtain the maximum cargo for this route.

$$
\operatorname{maxCargo}=\operatorname{maxCargo}+\text { extraFuel }=55,000+117,865=172,865
$$

The calculated values for the critical leg in the example are graphically summarized in Figure 3.7. 


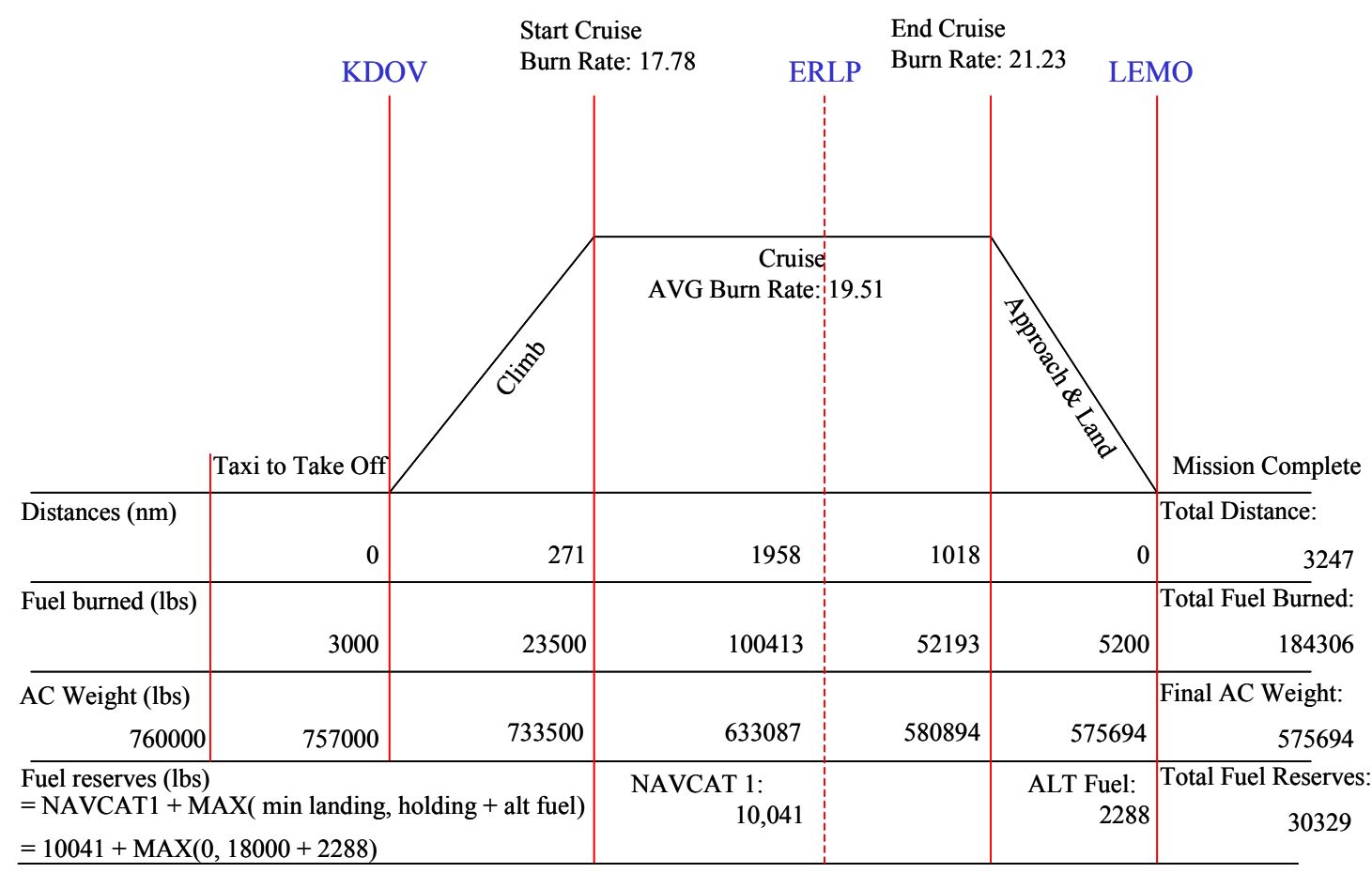

Figure 3.7 Example Maximum Cargo Calculation for a C-5A

\subsubsection{Calculating Fuel Requirements for a given payload}

Calculating the fuel requirements for a particular flight leg and a specific payload (rather than the maximum payload) is significantly more complex. An iterative procedure is used that varies fuel values until the minimum fuel required to fly the leg for the given payload is determined. The procedure employed to "guess" required fuel levels is a binary search. At the outset of the search the fuel required is set at the midpoint of zero fuel and the aircraft's fuel capacity. Calculations similar to those demonstrated above are then used at each step to determine if there is an excess or lack of fuel to complete the trip with the given 
payload. For an excess amount of fuel, the upper half of the current range of fuel values is discarded and the binary search repeats with the lower half. For insufficient fuel, the lower half fuel range is discarded and the binary search continues with the upper half. This procedure yields the approximate minimum amount of fuel required to fly the leg for the given payload.

\subsubsection{Determining Aircraft Payloads}

Payload determination for an aircraft is based on the AMOS "liquid ton modeling" method. Key factors are available cargo (cargo \& commodity types), aircraft type, payload targets, and route maximum cargo constraints. The following example illustrates how a payload is determined. Figure 3.8 gives a set of available requirements for the KGRK/OKBK APOE-APOD pair. The assigned aircraft is a C-5A.

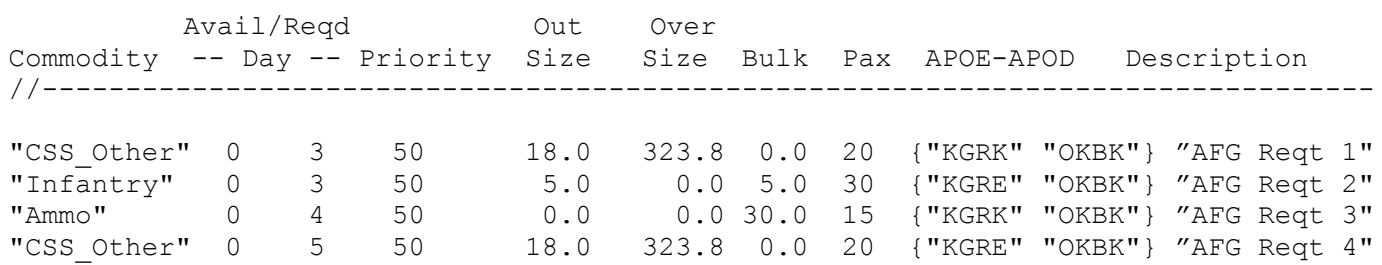

Figure 3.8 Extract from Requirements File

When ALDs, RDDs and priorities are identical, the default ordering for a set of requirements is the TPFDD ordering. Here there are four different commodities, each with different payload targets. The Table 3.1 route is also used for this example. Thus, the maximum cargo for the route is 172,865 pounds (86.4 tons). The example requirements ALDs are identical with different RDDs. 
Loading begins with Requirement 1 . Because a C-5A is compatible with the available outsize cargo, the first three values for type commodity CSS_Other in the payloadtgt.dat file will be used. From Figure 3.1, the maximum values for type commodity CSS_Other are as follows: 34.5 tons outsize, 16.9 tons oversize, 1.4 tons bulk and 71 personnel (PAX). Based upon the available quantities and maximum table values, 18 tons of outsize, 16.9 tons of oversize and 20 PAX are loaded. Note that PAX are loaded first and their weight is determined by using a standard PAX weight of 400 pounds defined in the typeac.dat file. Additionally, cargo maximum values are predicated on loading to the limit of the PAX fill-71 PAX in this instance. Thus far then, the total cargo loaded is 34.9 tons and PAX weight is 4 tons for a total payload of 38.9 tons, well below the route maximum cargo value.

The next step is to determine the percent payload capacity remaining for the aircraft. For PAX it is simply the number of seats remaining-51 seats in this instance. For cargo, the percent of capacity filled is the ratio of cargo loaded to the maximum cargo capacity defined by the table values.

$$
\% \text { Filled }=34.9 / 52.8=66 \%
$$

Thus $34 \%$ of cargo capacity remains to load items from the next requirement.

Because there is remaining capacity and maximum cargo limitations have not been exceeded, loading continues with Requirement 2. Again because outsize cargo is available, the first three columns of the payload target table (Figure 3.1) for commodity Infantry define maximum cargo values: 34.1 tons outsize, 16 tons oversize and 0.1 tons of bulk. PAX are loaded first with all 30 PAX available 
being loaded leaving 21 seats remaining. The maximum table values must be adjusted since only $34 \%$ of cargo capacity remains. Maximum table values are therefore reduced to $34 \%$ of their original value and the new limits become: 11.59 tons of outsize, 5.44 tons of oversize, and 0.03 tons of bulk. Thus, cargo loaded for Requirement 2 is 5 tons of outsize and 0.03 tons of bulk. The Requirement 2 payload weight total is 11.03 tons (includes PAX). Total payload is now 49.93 tons which is less than the route maximum cargo.

The percent fill for Requirement 2 is determined as follows:

$\%$ Filled $=5.03 / 17.06=29 \%$

Thus, assuming only Requirement 2 is loaded, the remaining capacity is $71 \%$. However, Requirement 1 is already loaded, so the true capacity remaining is $71 \%$ of $34 \%$. Thus $24 \%$ of cargo capacity remains.

Requirement 3 is now considered for loading. All 15 PAX are loaded leaving 6 seats empty. Because there is only bulk cargo available, the sixth column of the payload target table (Figure 3.1) for the type commodity Ammo defines the maximum cargo: 90 tons of bulk. Again, this must be reduced to reflect the cargo capacity remaining. The maximum bulk that can be loaded is $24 \%$ of 90 tons of bulk or a maximum value of 21.6 tons of bulk can be loaded. Since there are 30 tons available, all 21.6 tons of bulk is loaded. The total weight of Requirement 3 items loaded is 24.6 tons. The total current payload is now 74.53 tons. The percent fill is $100 \%$ since the maximum bulk value was loaded. No cargo capacity remains. 
Since PAX seats are available, loading proceeds with Requirement 4 . No cargo can be loaded and only 6 of the 20 PAX available are loaded. The final total payload is 75.73 tons. The aircraft is fully loaded and proceeds from the APOE to the APOD at OKBK. It is possible that this process will load trivial amounts of requirements as the percent of remaining capacity becomes small. To prevent this, a user defined percent capacity threshold is enforced. (The default value is $5 \%$.)

\subsubsection{SAP Building Blocks: Missions}

In solving the SAP, we want to find the best allocation of aircraft to requirements and the best routing and scheduling of aircraft so that late deliveries and non-deliveries are minimized and essential constraints are not violated. Since a SAP solution is a set of missions flown by the various aircraft, a mission is the basic solution building block.

Each mission has an associated APOE/APOD pair. A typical mission consists of flight segments sequencing through home base, APOE, APOD, recovery base and then back to home base. Each flight segment traverses an appropriate route (defined in the plan file). Upon return to home base, the aircraft undergoes maintenance and refueling, and then is available for subsequent missions. There are variations on this basic mission pattern. For example, subject to the return to base constraint, an aircraft could move from a recovery base directly to an APOE and start a new mission instead of returning to home base. 


\subsubsection{Determining Timings for a Mission}

Each mission consists of a set of route segments, each with a defined origin and destination. Within a route segment there are one or more flight legs. The timings for a mission are determined by combining the timings for all the flight legs that make up the mission, i.e., the flight leg is the fundamental component of a mission.

As an example of computing the timings of a flight leg, consider the critical leg used in the maximum cargo calculation example (KDOV-ERLPLEMO) for a C-5A. Suppose the payload is set to the maximum cargo found in the example, 86.4 tons and takeoff from $\mathrm{KDOV}$ is at time zero. A graphical depiction of the flight leg, with segment times, is presented in Figure 3.9.

Upon takeoff, the aircraft climbs to its cruising altitude, the climb segment. The time to reach altitude (31,000 feet in this example) based upon a given aircraft weight is defined in the climb section of the fuelcalc.dat file (see

Figure 3.5 above). In this example, the climb weight of the aircraft is 757,000 pounds which yields a climb time of 45 minutes. 


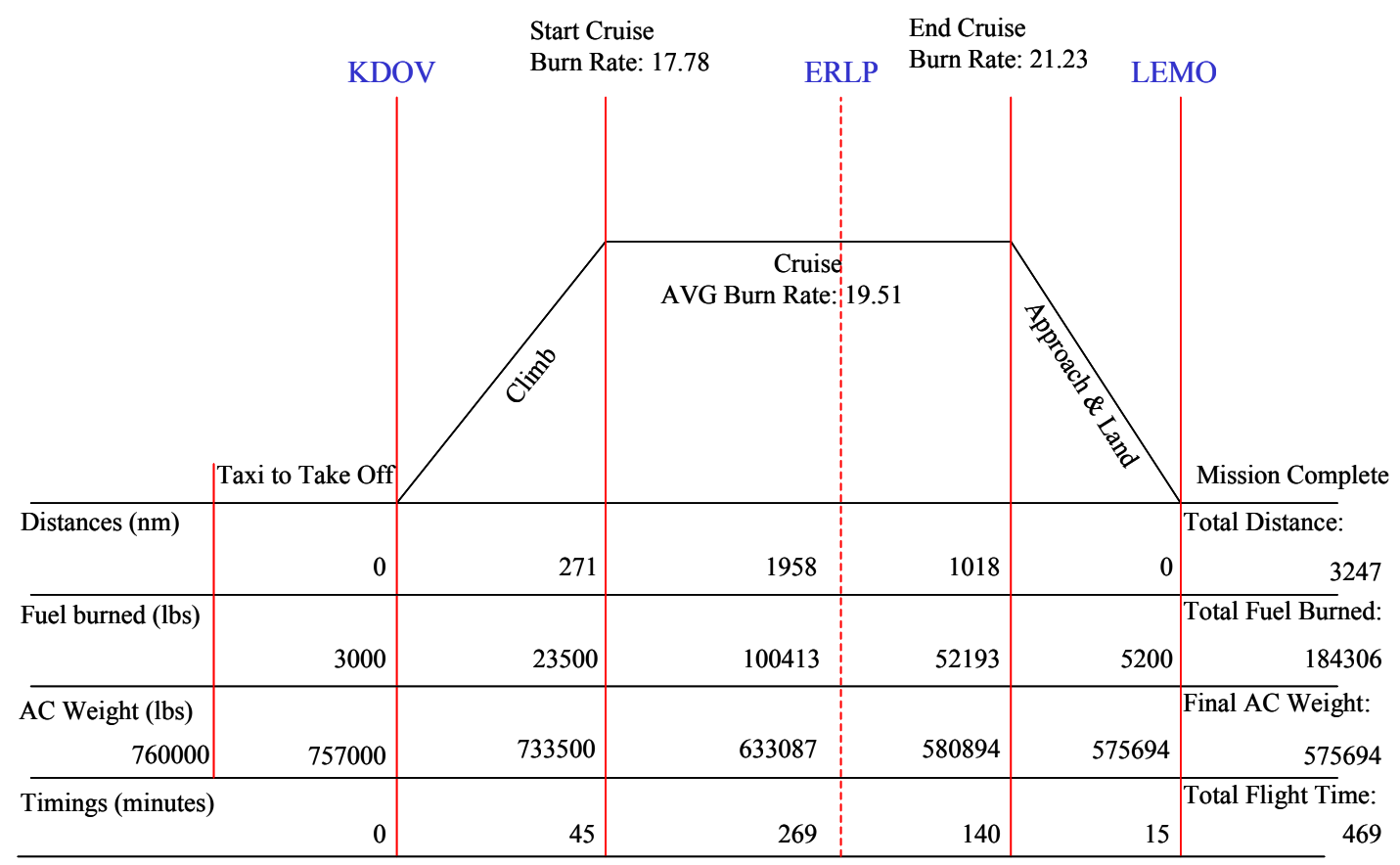

Figure 3.9 Example Flight Leg Timings

The aircraft now enters the cruise segment of the flight leg. The standard cruising speed for a C-5A is 0.74 mach. Computation of the cruise time, requires conversion of the true air speed into knots according to accepted formulae (Williams, 2002). Required inputs are the Mach number (MachNum), the temperature at zero altitude (zeroTemp) in degrees Celcius and the altitude (alt) at the Mach number (hundreds of feet). For our example, the Mach number is 0.74 , the zero altitude temperature is set at $18.33^{\circ} \mathrm{C}$ (about $65^{\circ} \mathrm{F}$ ), and the altitude is 310 hundred feet. The many factors used to obtain the constants used in the formulae below are defined by Williams (2002). The computation proceeds as follows:

$$
\begin{aligned}
\text { temp } & =\text { zeroTemp }-(0.0019812 *(\text { alt } * 100)) \\
& =18.33-\left(0.0019812 *\left(310^{*} 100\right)\right)=-43.08^{\circ} \mathrm{C}
\end{aligned}
$$




$$
\begin{aligned}
\text { TAS } & =\text { MachNum } *(38.967854 * \sqrt{\text { temp }+273.15}) \\
& =.74 *(38.967854 * \sqrt{-43.08+273.15}) \\
& =437.38 \text { knots }
\end{aligned}
$$

Once the true air speed (TAS) is known, cruise times are calculated as follows:

$$
\text { cruise Time }=60 * \text { cruiseDist } / \text { TAS }
$$

For the first cruise portion to ERLP, the cruise time is cruiseTime $=60 * 1958 / 437.38=268.60$ minutes.

In a similar way, cruise time from ERLP to LEMO is calculated to be 139.65 minutes for a total cruise time of 408.25 minutes. As in AMOS, the cruise distance used places the aircraft directly above the destination. A standard approach and landing time is then added (currently set at 15 minutes) to obtain the total flight time from KDOV to LEMO.

Since LEMO is an en route base, the aircraft will undergo refueling and routine maintenance checks before embarking on the next leg. En route times are defined in the typeac.dat file. For a C-5A, the en route time is 3.25 hours or 195 minutes. In summary, the total flight time for this leg is 469 minutes and the total ground time at LEMO is 195 minutes.

Standard en route times are also used to model delays at recovery bases and home bases. There are separate standard times set for onloading and offloading. Timing for an entire mission is determined by summing the timings for the various legs that make up the mission.

\subsubsection{An Example Mission}

To illustrate how a mission is built using the various input files, suppose that there is a requirement of commodity type CSS_Other consisting of 50 tons of 
outsize, 12 tons of oversize and 15 tons of bulk to be moved from Robert Gray Army Airfield in Texas (KGRK, Region 20) to Kuwait International Airport in Kuwait (OKBK, Region 33). Since there is outsize cargo available, a C-5A would be a suitable choice. Assume that the C-5A home base is at Dover Air Force Base in Delaware (KDOV, Region 21). The recovery base for a C-5A in Region 33 is at Moron Airbase, Spain (LEMO, Region 24). For this mission, the aircraft will go from KDOV to KGRK to OKBK to LEMO and back to KDOV.

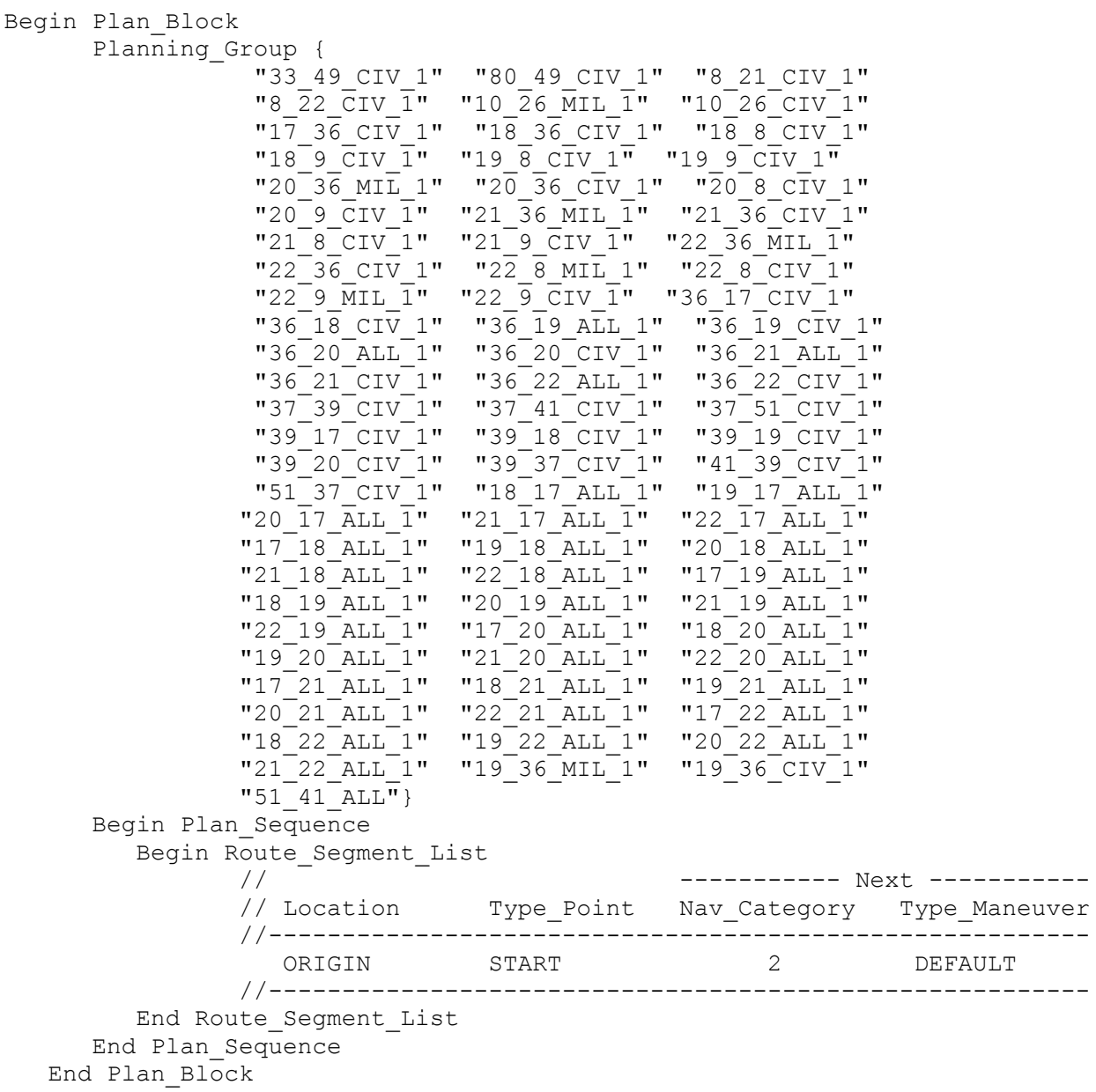

Figure 3.10 Route Segment Joining Region 21 to Region 20 
For the first leg, the regions are 21 and 20 . The plan document route segment joining these regions is shown in Figure 3.10. This route segment indicates a direct flight from KDOV to KGRK (no intermediate bases or waypoints).

At KGRK, the amount of cargo that can be loaded is a function of the requirements available, the payload targets defined in the payloadtgt.dat file, and the limitations imposed by the route. The payload targets for commodity type CSS_Other for a C-5A are given in Figure 3.11.

\begin{tabular}{|c|c|c|c|c|c|c|c|c|}
\hline $\begin{array}{ll}/ / & \text { Type } \\
/ / & \text { Commodity }\end{array}$ & Out $>0$ & Over $>\overline{0}$ & $\mathrm{Bulk}>0$ & $\begin{array}{r}\text { Over }>0 \\
\text { Out }=0\end{array}$ & $\begin{array}{l}\text { Bulk } \\
\text { Out }=0\end{array}$ & $\begin{array}{c}\text { Bulk } \\
\text { Both=0 }\end{array}$ & $\begin{array}{l}\text { Pax } \\
\text { Fill }\end{array}$ & $\begin{array}{l}\text { Pax } \\
\text { Only }\end{array}$ \\
\hline //---------- & ------ & ------ & ------- & -------- & ------- & --------- & ------ & ----- \\
\hline "AF_Acft" & 41.3 & 20.6 & 0.0 & 51.1 & 1.2 & 68.4 & 71 & 340 \\
\hline$\cdot$ & $\cdot$ & $\cdot$ & $\cdot$ & $\cdot$ & $\cdot$ & $\cdot$ & $\cdot$ & $\cdot$ \\
\hline$\cdot$ & $\cdot$ & $\cdot$ & • & $\cdot$ & $\cdot$ & - & - & $\cdot$ \\
\hline$\cdot$ & $\cdot$ & $\cdot$ & · & $\cdot$ & . & . & - & . \\
\hline "CSS_Other" & 34.5 & 16.9 & 1.4 & 66.2 & 5.9 & 82.8 & 71 & 340 \\
\hline "Typē_24" & 34.5 & 17.9 & 0.4 & 62.1 & 9.0 & 78.3 & 71 & 340 \\
\hline "Resupply" & 34.5 & 16.7 & 1.6 & 64.0 & 7.6 & 82.8 & 71 & 340 \\
\hline "Ammo" & 0.0 & 0.0 & 0.0 & 0.0 & 0.0 & 90.0 & 0 & 340 \\
\hline
\end{tabular}

Figure 3.11 Extract from Payload Target File for C-5As

The first three columns define what can be loaded for this commodity and aircraft type- 34.5 tons of outsize, 16.9 tons of oversize and 1.4 tons of bulk. Thus, the payload should be 34.5 tons of outsize, all 12 tons of the oversize, and 1.4 tons of bulk for a total payload of 47.9 tons. Next, we must confirm whether the chosen route segment's maximum cargo can support 47.9 tons.

Determining a route maximum cargo begins with identifying the route between the APOE and APOD (KGRK and OKBK). Since planning group 
20_33_C-5S_1 joins KGRK (Region 20) to OKBK (Region 33), we could use the route segment in Figure 3.3. As derived earlier, the route's maximum cargo of 86.43 tons exceeds the payload target total of 47.9 tons.

Following offloading at $\mathrm{OKBK}$, the aircraft proceeds to recovery base LEMO. Figure 3.12 presents one of the route segments joining OKBK to LEMO.

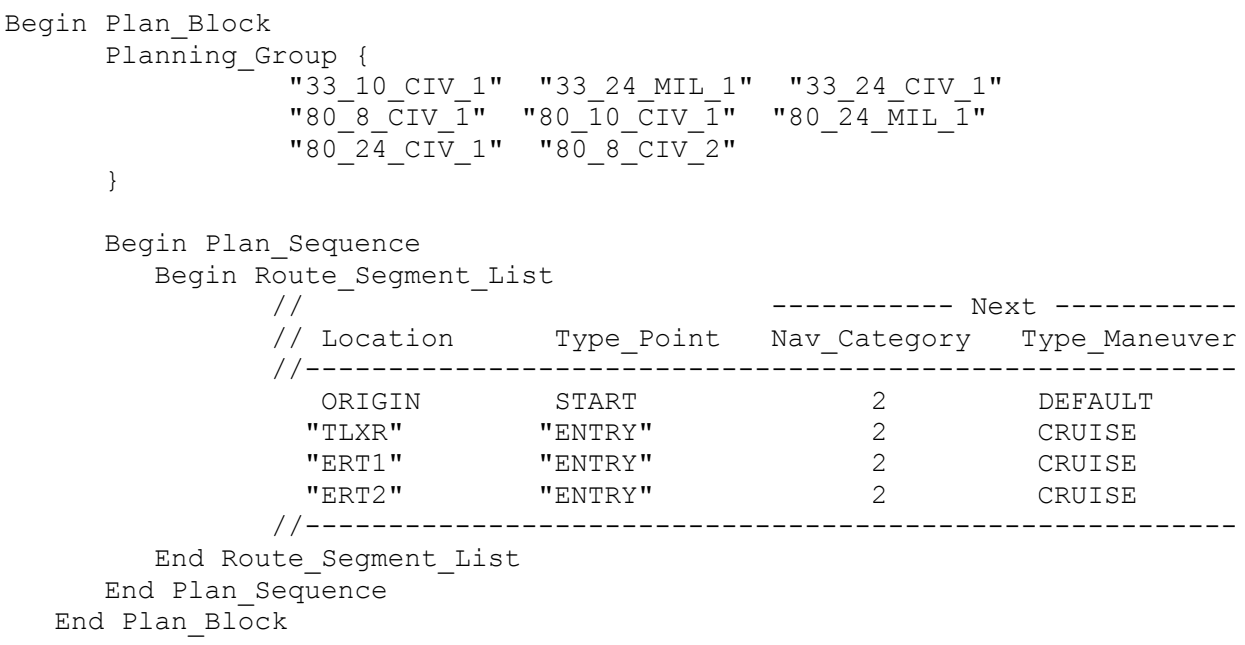

Figure 3.12 Route Segment for OKBK to LEMO

If this route is selected, the aircraft proceeds to its recovery base via OKBK-TLXR-ERT1-ERT2-LEMO. Following required maintenance and refueling at LEMO, the aircraft returns to its home base. One route segment joining LEMO to KDOV is shown in Figure 3.13. Note that the destination, $\mathrm{KDOV}$, is a part of the route segment. If this route is selected, the aircraft flies directly from LEMO to KDOV. 


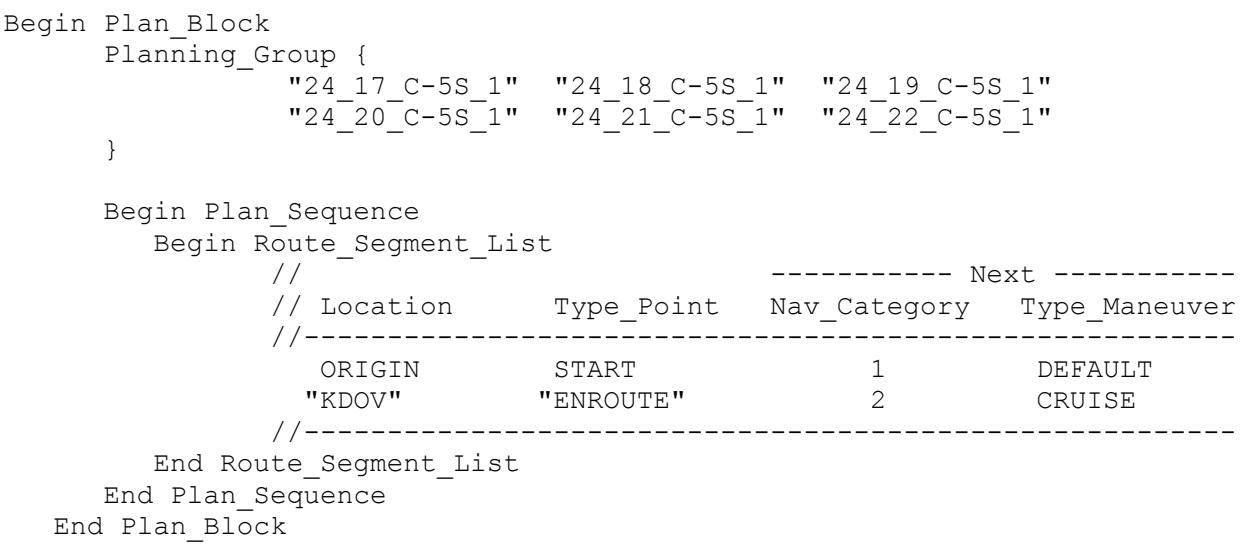

Figure 3.13 Route Segment for LEMO to KDOV

In summary, the example mission traverses the following points: (KDOV, KGRK, KDOV, ERLP, LEMO, ERT2, ERT1, TLXR, OKBK, TLXR, ERT1, ERT2, LEMO, KDOV).

\subsubsection{SAP Decisions}

For a given SAP instance, a solution requires the following decisions:

- The detailed asset level visibility of aircraft and TPFDD level visibility of requirements (aircraft/requirement pairings).

- The selected detailed routings flown by each aircraft in servicing an APOE/APOD pair (aircraft missions).

- Time phasing of aircraft along routes, both to the APODs and on return flights, to account for the dynamically changing network characteristics (for example, changing available MOG due to the arrival and departure of aircraft). This includes the takeoff and landing times of each aircraft at the APOEs, APODs and at recovery and en route bases. 
To facilitate time phasing decisions, it is assumed that AMC can dictate aircraft departure times and vary flight speeds. The SAP objective is multicriteria and hierarchical in nature. Depending upon mission priorities, pertinent criteria and their ordering may change. Key objectives include minimizing the lateness of all requirements at their APOD, minimizing congestion at airfields in the airlift network, minimizing the number of missions flown, and minimizing the number of aircraft used.

The above set of inputs and decisions represent the basic SAP model. Aircraft are assigned a series of missions to cover the given requirements. Aircraft may return to home base at the end of each mission, or within the defined return to base time, an aircraft may move from its recovery base to an APOE. The following section discusses the SAP representation in the context of the chosen solution methodology.

\subsection{SAP REPRESENTATION}

One goal in formulating our representation is to compactly synthesize the critical SAP aspects so that construction and implementation of moves in a TS framework are facilitated. The inherent ordering already present for each SAP mission strongly effects this consideration. 


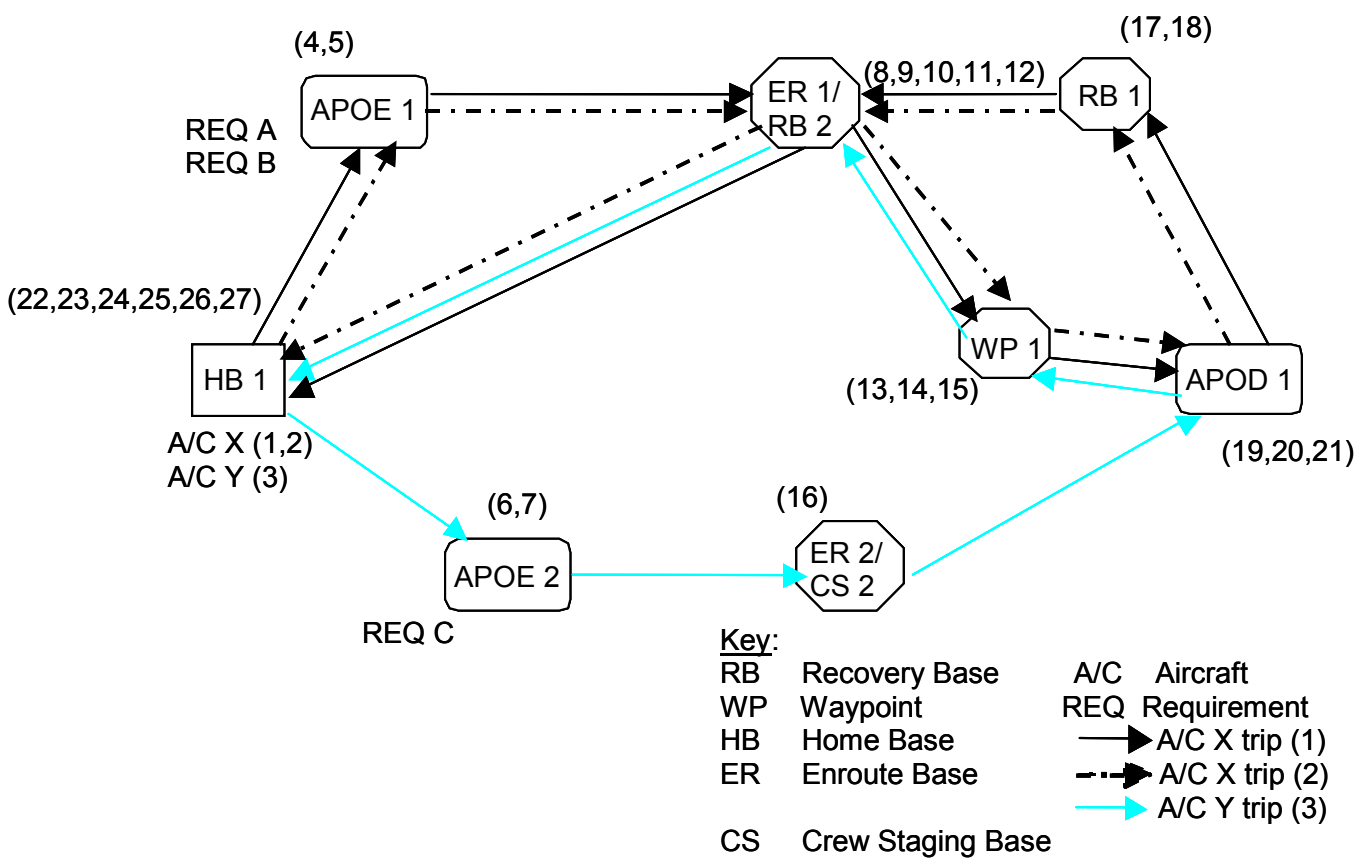

Figure 3.14 SAP Representation using Pure Nodal Approach

\subsubsection{Pure Node Based Approach}

Representing the SAP using a pure nodal approach is the most atomic representation. In this representation, every point visited by an aircraft in a mission would be assigned a node. Figure 3.14 depicts a simple instance of a SAP airlift network. There are three requirements (A, B \& C) located at two different APOEs. Arrows indicate a possible solution using feasible paths through the network. The numbers at each node represent a separate visit to that particular node. Thus, using the pure nodal approach, the solution in the figure may be represented in the following manner: 
$(1,22,4,8,13,19,17,9,23)(2,24,5,10,14,20,18,11,25)(3,26,6,16,21,15,12,27)$.

Each subset of numbers (within the parentheses) represents a mission. The succeeding numbers represent points visited during the mission. In the first mission, aircraft 1 is used. It departs HB1(represented by 22) and proceeds to APOE1(4). There, some portion of requirements A and/or B are loaded. Payload attributes would be stored with the aircraft trip number. Using the route segment represented by ER1(8) and WP1(13), the aircraft proceeds to its destination APOD1(19) where the cargo is offloaded. The aircraft then flies to its recovery base, RB1(17). Finally, the aircraft flies via the route segment ER1(9) to its home base HB1(23) where it prepares for subsequent missions.

For realistic instances of the SAP, the cardinality of the pure nodal representation will be very large. Because locations are often visited multiple times by many aircraft, a unique node for every visit would have to be created to capture such attributes as when an aircraft passed through a node. Additionally, there is an inherent structure and ordering for a SAP mission. For example, route segment sequences must be followed and APOEs must be visited prior to APODs. Thus, in an ATS sense, a move that swaps nodes within a route segment or puts an APOD prior to an APOE are inadmissible. To change a route segment under this representation, a string of nodes would have to be removed and a new string inserted. Most importantly, this representation does not exploit the tremendous reduction in possible routes due to the region concept. Thus the large cardinality 
and complexity of dealing with the SAP using a pure nodal approach outweighs the potential gains due to its atomic nature.

\subsubsection{Arcs}

A fundamental fact concerning the manner in which the airlift system is built and modeled at AMC is that there are a restricted number of ways to get from one region to another. The plan file lists all possible route segments joining two regions for defined aircraft groups (Military, Civilian, C-5s, etc.). The use of regions dramatically reduces the number of possible routes. Thus, instead of using a purely nodal approach, consider the use of a supernode or arc as an aggregate representation of a set of nodes. For our purposes, arcs will have an origin node, a route segment (comprised of a series of bases and waypoints) and a destination node.

In every mission, there is an origin location for the aircraft (normally the home base), an APOE, an APOD, a recovery base, and an ultimate destination (normally home base). Joining these locations are the route segments. Thus, for a typical mission, there is an arc for the home base to APOE node set, an arc for APOE to APOD, an arc for APOD to recovery base, and an arc for recovery base back to home base. Consider the first mission in the pure nodal approach example presented in the previous section. That nine node mission would now be represented by five objects: a node for the aircraft trip, and an arc each for HB1 to APOE1, APOE1 to APOD1, APOD1 to RB1 and RB1 to HB1. Figure 3.15 shows the missions pictured in Figure 3.14 using the arc representation. 


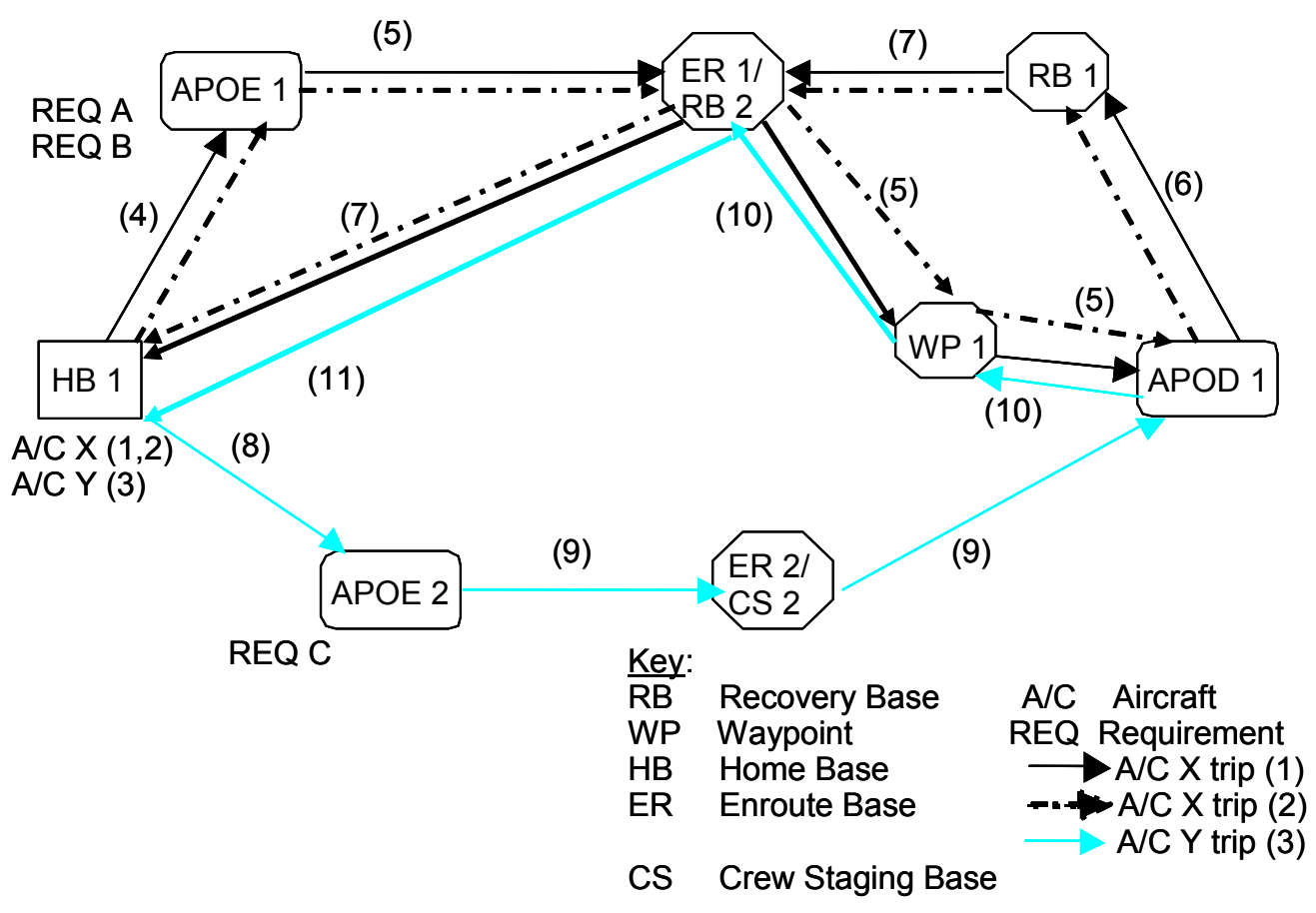

Figure 3.15 SAP Representation using Arcs

Numbers next to directed arrows represent the Arc object that contains the nodes connected by the arrow. The three mission solution representation now is:

$$
(1,4,5,6,7)(2,4,5,6,7)(3,8,9,10,11) \text {. }
$$

This representation exploits the structure of the airlift network and the structure of typical airlift missions, providing a much more compact representation than the pure nodal approach. This representation is used throughout this dissertation. Arc attributes and other details concerning the SAP representation and data structures are described in Chapter 4. 


\subsection{SUMMARY}

This chapter addressed the AMOS input file structure, key SAP decisions and the SAP representation. The key decisions for the SAP are the assignment of aircraft missions to requirements and the routing and scheduling of the assigned missions. The arc-based SAP representation compactly captures mission assignment information (mission node) and routing and scheduling information (arcs). The structure of a typical mission using this representation facilitates the implementation of a tabu search methodology. Chapter 4 provides a detailed description of the tabu search algorithm developed based upon this representation. 


\section{Chapter 4: A Tabu Search Approach to the Strategic Airlift Problem}

This chapter describes the Strategic Airlift Problem Tabu Search algorithm (SAP-TS).

\subsection{TABU SEARCH ARCHITECTURE}

Basic tabu search was described in Chapter 2. The ATS approach developed for the SAP incorporates several features to enhance the search. These features are: an adaptive memory feature that varies the tabu tenure to encourage intensification in promising areas and diversification in less promising regions; the use of dynamic neighborhood selection to guide the choice of neighborhood based upon the search trajectory; and, once the neighborhood is selected, the aggressive use of candidate list strategies to target moves that are most likely to improve solution quality. These features along with the TS framework employed are described in detail below.

\subsubsection{JAVA ${ }^{\mathrm{TM}}$ Software Programming Language and OpenTS}

The SAP tabu search algorithm was developed using the Java ${ }^{\mathrm{TM}}$ software programming language. The portability and object-oriented nature of the Java ${ }^{\mathrm{TM}}$ programming language were key factors. Additionally, prior efforts by Harder (2000), Wiley (2001) and Crino (2002) illustrate the efficacy of Java ${ }^{\mathrm{TM}}$ in rapidly developing effective tabu search algorithms. In particular, Harder's Java ${ }^{\mathrm{TM}}$ implementation of a generalized tabu search engine, OpenTS, provides the framework within which specialized tabu search algorithms can be developed. In 
OpenTS, the user defines the basic components of tabu search in the context of the problem. The OpenTS required components along with the associated SAPTS classes are shown below (Figure 4.1).

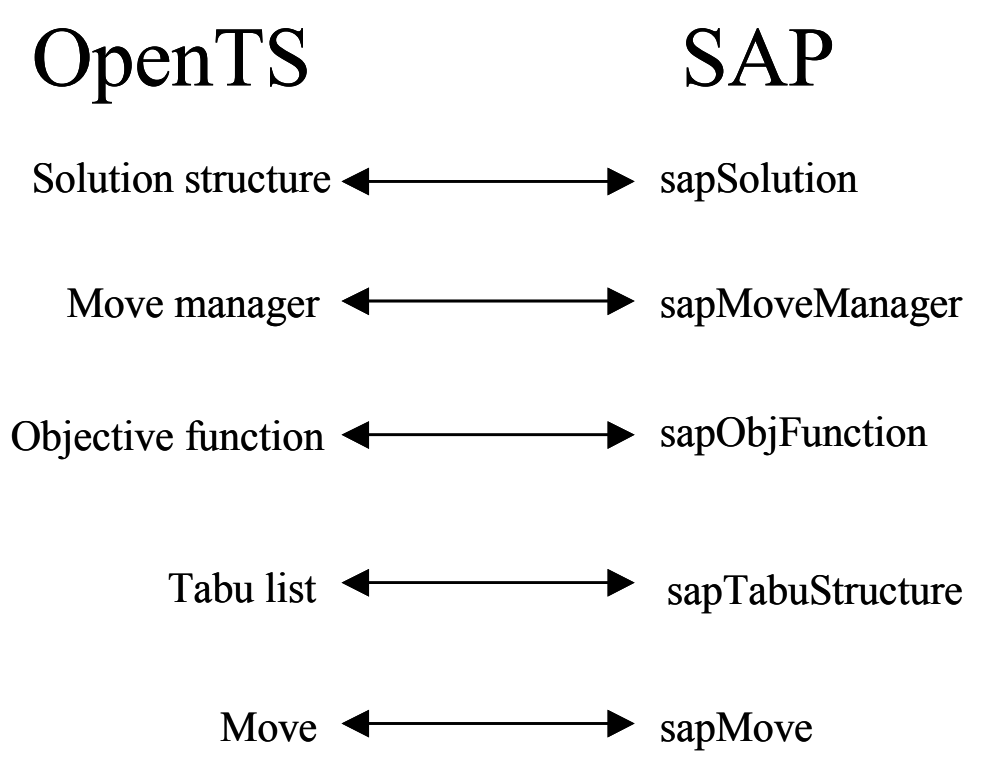

Figure 4.1 OpenTS Required Elements and Associated SAP-TS Classes

An iteration in OpenTS starts with an incumbent solution. Given a starting or current solution, the move manager is then called and generates a list of moves for consideration. These moves are then invoked in turn on the incumbent solution and sent to the objective function object for evaluation. Using the tabu list, the best non-tabu move is selected. High quality solutions that are tabu may still be selected using some aspiration criteria such as "best solution found so far", to override the tabu status. Once a move is selected, the move is performed on the incumbent solution and the new solution is passed to the move 
manager to generate the next set of moves. Figure 4.2 shows this process graphically.

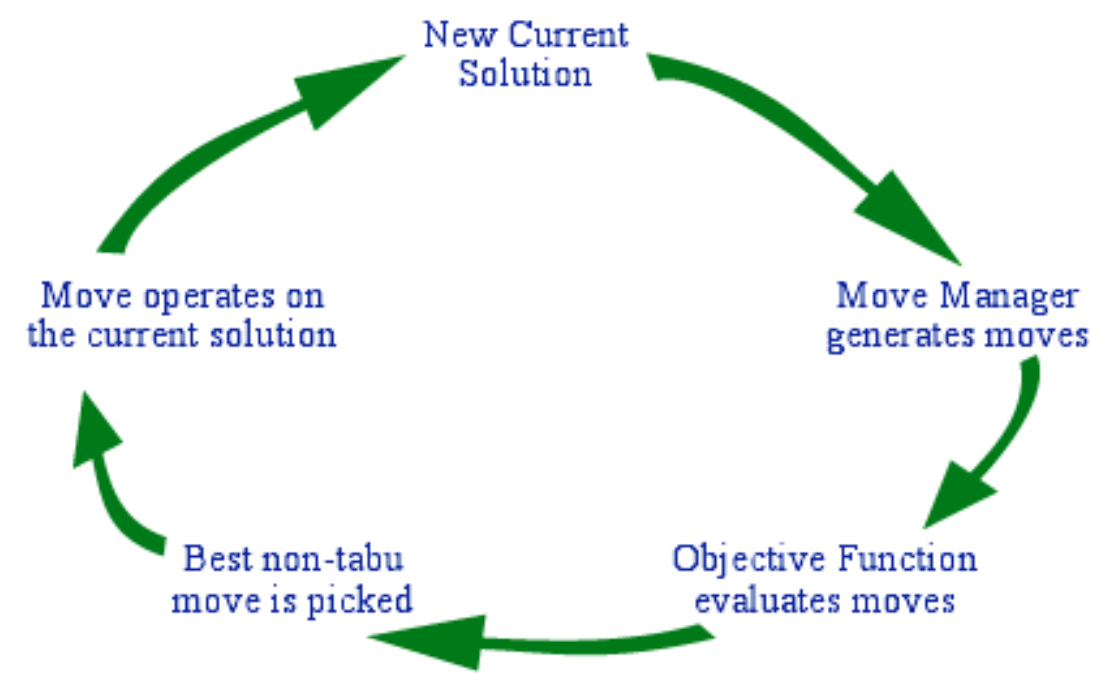

Figure 4.2 OpenTS Architecture (Harder 2000)

\subsection{SAP-TS DATA STRUCTURES}

During preprocessing, AMOS data input files are read and a series of objects created. These objects and their key attributes are summarized in the table below. 


\begin{tabular}{|l|l|}
\hline Object & Key Attributes \\
\hline AC & $\begin{array}{l}\text { Type, weights, times, fuel data, payload targets, } \\
\text { cargo compatibility }\end{array}$ \\
\hline Aircraft & $\begin{array}{l}\text { Tail number, Home base, unit, missions, RTB time, } \\
\text { time available, last time at HB }\end{array}$ \\
\hline Locations & $\begin{array}{l}\text { ID number, Lat-Lon, region, narrow working } \\
\text { MOG, wide working MOG, MOG logic, daily fuel } \\
\text { capacity, fuel deliveries }\end{array}$ \\
\hline Requirements & $\begin{array}{l}\text { Requirement number, ALD, RDD, APOE, APOD, } \\
\text { commodity type, outsize, oversize, bulk, PAX }\end{array}$ \\
\hline Routes & $\begin{array}{l}\text { Source Region, Sink region, planGroupName } \\
\text { ID number, Start base, stop base, Route segment, } \\
\text { Arc }\end{array}$ \\
\hline legs, leg timings, leg fuelRequired, leg fuelBurned \\
Mode
\end{tabular}

Table 4.1 SAP-TS Objects and their Attributes

Available aircraft units define the number, type, home base (HB), and available time for the available aircraft. An $A C$ object is created for each type of aircraft available. An Aircraft object is created for each physical aircraft available for the instance. Aircraft objects inherit the attributes of the associated $A C$ object. A Locations object is created for each base and waypoint available in the airlift 
system. A Requirements object is created for each requirement listed in the requirements file. The set of available APOEs and APODs and their unique pairings are defined by the list of available requirements. The set of possible recovery bases (REC) is based upon the set of APODs and aircraft types available - one or more REC bases for each APOD and aircraft type available.

The set of HBs, APOE to APOD pairs, and REC bases generated above are used to construct the Arc objects. The set of possible Arc types is comprised of HBtoAPOE Arcs, APOEtoAPOD Arcs, APODtoREC Arcs, RECtoHB Arcs, and RECtoAPOE Arcs. An Arc object is generated for each of these types based upon all available bases (HB, APOE, APOD, REC) and the different route segments (defined in the Routes object) connecting the endpoint bases. Arcs feasible for more than one aircraft type store unique timings and fuel requirements for respective aircraft types. The number of Arc objects generated for a particular Arc type, say HBtoAPOE, is:

$$
\sum_{i \in\{H B s\}} \sum_{j \in\{\text { APOEs }\}} \sum_{k \in\{\text { Segments }\}} I\left(H B_{i}, \text { APOE }_{j}, \text { Segment }_{k}\right)
$$

where $I\left(H B_{i}, A P O E_{j}\right.$, Segment $\left._{k}\right)$ is the indicator function that has value one if Segment $_{k}$ joins $H B_{i}$ to $A P O E_{j}$ and value zero otherwise.

The total number of Arcs generated is the sum over all the Arc types. For an instance of moderate size containing 10 HBs, 48 APOEs, 30 APODs, with 56 unique APOE-APOD pairs, and 18 RECs, over 11,000 Arcs were generated. To keep the number of Arcs generated at a reasonable level, RECtoAPOE Arcs are 
generated only as needed during the course of the search since return to base (RTB) times limit the number of missions that can employ RECtoAPOE Arcs.

The number of aircraft mission Node objects generated is a balance between ensuring sufficient missions are available for each aircraft while not having so many that the search is degraded. The default number of mission Node objects generated for each aircraft allows the aircraft to be active for about one third of the planning horizon. This is based on a typical mission cycle time of just over two days.

\subsection{SAP-TS SOLUTION STRUCTURE}

As described in Section 3.2.2, a solution to an instance of the strategic airlift problem is comprised of a set of missions. The sapSolution object holds the set of assigned missions in an array. Each mission is an array containing the identifiers that point to the Node and Arc objects that hold assigned payload, timings and fuel consumption as well as the routing information. Missions are ordered in the solution by aircraft number and then temporally for each mission assigned to the aircraft.

Recall that a typical mission starts from a HB, travels to an APOE and loads cargo, flies to an APOD and offloads the cargo, moves to a regional REC base and then returns to the HB. A mission Node contains information about the departure and arrival times for the HB, APOE, APOD, REC and HB bases along the route, a detailed breakdown of the payload (requirement number and quantity of each cargo category), and the percent capacity remaining for the aircraft. Four separate Arc objects capture the routing information and standard times and fuel 
requirements for arcs with no payload. The mission Node holds the timings and fuel requirements for the APOE-APOD arc based upon the payload. For a standard mission then, the mission array structure is:

(MissionNode\#, HBtoAPOE\#, APOEtoAPOD\#, APODtoREC\#, RECtoHB\#)

A collection of these missions constitutes a solution to the SAP.

\subsection{INITIAL SOLUTION CONSTRUCTION}

The initial solution is constructed incrementally using a greedy method to assign aircraft missions to APOE-APOD pairs. Available missions are sorted according to time available. The first mission for each aircraft is assigned an available time equal to the original time available for the aircraft. Subsequent missions for the aircraft are assigned later available times (artificially) by adding an additional day to the original available time. Requirements are sorted and grouped by APOE-APOD pair and ALD. The pseudocode for this procedure is presented in Figure 4.3. 
Pseudo code for initial solution

Sort aircraft missions by time available

Initialize requirement array

for each day $i$

$\{$ if no aircraft missions left, break

for each APOE-APOD pair $j$

\{ while cargo remaining for day $i$ and APOE-APOD pair $j$

$\{$ while mission not assigned

$\{$ get next aircraft mission

if not compatible with available cargo, continue

else

$\{$ assign the mission

remove mission from mission candidate list, set mission assigned $=$ true

\}//end else

\}//end while mission not assigned

//build the mission

select APOE-APOD Arc id

while cargo left $\cap$ space left on day $i$ at APOE-APOD pair $j$

\{ load cargo available

\}//end while

build mission route

update aircraft time available

add mission to solution array

if no aircraft missions left, break

\}end while cargo remaining

\}$/ /$ end for $j$

\}//end for $i$

Figure 4.3 Pseudo Code for Initial Solution

The initial solution construction method quickly yields a solution to the

SAP that is typically an infeasible but suitable starting point for SAP-TS. 


\subsection{OBJECTIVE FUNCTION}

The SAP-TS objective function object calculates the current solution value based upon the following criteria:

- Undelivered cargo

- Late cargo

- Working MOG violations

- Fuel MOG violations

- UTE Rate violation

- Trivial load violations

- Number of trivial loads

- Number of missions

- Number of aerial refueling missions

- Total objective value

Undelivered and late cargo measure how well a solution meets RDDs for a given SAP instance. Undelivered cargo is the total weight (tons) of all cargo not delivered to their respective APODs. Late cargo is the total weight (tons) of all cargo not delivered to their respective RDDs. Both undelivered cargo and late cargo are weighted according to the amount of time (in days) that the cargo is late. The "amount late" for the undelivered cargo is dependent on the user specified planning horizon. The weight for late cargo is the difference between the arrival time at the APOD and the cargo's RDD plus one--delivery anytime on the RDD is considered "on time". 
Working MOG, fuel MOG and UTE rate violations measure the feasibility of a solution. Working MOG violations are the sum of the total number of MOG violations at each base at each time interval. The default time interval is 15 minutes. Fuel MOG violations are the sum of the daily fuel shortages (in tons) at all bases under the current solution. A solution with a feasible UTE rate has no more aircraft in the air at any given time than the respective aircraft UTE rates. The UTE rate violation is the sum of the difference between of the number of aircraft of each type in the air at any given time and the particular aircraft type's UTE rate. Working MOG and fuel MOG violations indicate overly optimistic throughput of aircraft in the airlift network for a given solution. UTE rate violations indicate overly optimistic aircraft operational rates. The default feasible settings for these measures are 0. Experience with feasible AMOS solutions to SAP instances show that these measures are rarely 0 - especially for MOG and UTE measures. Thus, user defined threshold values for these measures are permitted in SAP-TS to preclude overly constraining the airlift system.

Trivial loads are loads whose weight falls below an aircraft's trivial load threshold. A mission with a trivial load is not permitted in AMOS. Depending on rules invoked by the user, trivial loads are either aggregated at a central base and then delivered or are simply not delivered. In the SAP-TS implementation however, trivial loads are permitted - all requirements are delivered. Thus the presence of trivial load missions does not violate feasibility of a solution. Missions with payloads less than the trivial load are counted and the trivial load 
violation, the sum of the difference between the trivial load threshold and the payload, is computed.

The remaining criteria incorporated into the objective function are simple counts. The number of trivial loads is a count of the number of missions whose payload is less than the aircraft's trivial load threshold. The number of missions is the current total number of assigned missions. The number of aerial fueling missions is the sum of all aerial refueling legs used in the current solution. Air Mobility Command desires to limit the number of Aerial Refueling missions in support of strategic airlift because refueling assets are dedicated to supporting deployment of fighters and bombers. This is especially true in the surge phase of a major deployment.

These criteria are combined using a weighted sum (default weights are 1 for each of the criteria) and the goal is to minimize this weighted sum. In Harder's OpenTS, objective function comparison is conducted by comparing each component of the objective in a hierarchical fashion until a "winner" is determined or all objective components have been checked. The order in which the SAP-TS objective components are compared is the objective total, undelivered cargo, late cargo, working MOG violation, UTE rate violation, fuel MOG violation, trivial load violation, number of missions, number of trivial loads, and the number of aerial refueling missions.

\subsection{MOVE NEIGHBORHOODS}

Critical decisions for the Strategic Airlift Problem are assignment of aircraft to requirements, routing of aircraft in assigned missions and the detailed 
timings of aircraft movement through the airlift network. The moves developed target aspects of the SAP that work to obtain feasibility and improve upon assignment, routing and scheduling decisions. This section describes the various move neighborhoods developed for SAP-TS.

\subsubsection{SAP-TS Neighborhood Phases}

The Strategic Airlift Problem can be broken into two phases-a mission assignment phase and a mission improvement phase. In the assignment phase, aircraft missions are allocated to service requirements at APOE-APOD pairs. A mission assignment/scheduler heuristic (described in section 4.9) is then called to

build and schedule missions based upon the given assignment. Once an assignment of an aircraft mission to an APOE-APOD pair is made, the mission improvement phase is invoked to obtain the "best" routing and timings for the given mission assignment scheme.

Thus, given a set of APOE-APOD pairs and a set of available missions, we seek a "good" assignment of these missions to the pairs that minimizes the objective function. Then, based upon the mission assignments, local changes to missions are made seeking further improvement. The New Mission Insert Move Neighborhood, Within Pair Insert Move Neighborhood, Between Pair Swap Move Neighborhood, and Between Pair Insert Move Neighborhood traverse the mission assignment space. The Within Mission Arc Swap Move Neighborhood, Load Reallocation Move Neighborhood, APOE to Recover Arc Insert Move Neighborhood and the Impose Time Delay Move Neighborhood work to improve 
attributes of individual missions. These neighborhoods are described in detail below.

\subsubsection{New Mission Insert Move Neighborhood (NMI)}

NMI moves target APOE-APOD pairs that have either undelivered or late requirements. Undelivered requirements (if any) are targeted first and are sorted according to largest undelivered quantity. Late requirements are then considered and are sorted according to the product of the quantity of cargo late (tons) and the amount late (days). Insert missions are selected from the set of current unused missions and inserted into the set of missions currently assigned to the respective APOE-APOD pairs. The number of candidate new missions is restricted to no more than one per available aircraft -inserting more than one mission for the same aircraft is redundant. Additionally, candidate new missions are checked for load feasibility for each undelivered or late requirement considered. For example, if an undelivered requirement under consideration consists only of outsize cargo and the current candidate new mission is a PAX only aircraft, the new mission is not considered for insert.

The number of possible insertion points for a given unused mission and APOE-APOD pair is one more than the current number of missions assigned. Even for moderate sized problems, the number of possible inserts can be prohibitively large. Thus a candidate list structure is imposed on the NMI neighborhood to focus on insert locations most likely to reduce undelivered or late requirements. Candidate insert locations for undelivered requirements are the locations of those current missions whose last requirement ALD is either "close" 
to the undelivered requirement ALD or whose payload contains some portion of the undelivered requirement. A mission's last ALD is "close" if it is within the user specified move distance used in the LR neighborhood (default is 3 days). Similarly, candidate insert locations for late requirements are the locations of those missions whose last ALD is within the LR move distance or whose payload contains some portion of the late requirement.

As an example, consider the mission number assignments presented in Table 4.2. In this example there are five aircraft available, each with five possible missions.

\begin{tabular}{|l|l|l|}
\hline Tail Number & Aircraft Type & Available Mission Numbers \\
\hline 1 & C-5A & 1 through 5 \\
\hline 2 & C-5A & 6 through 10 \\
\hline 3 & WBC & 11 through 15 \\
\hline 4 & WBP & 16 through 20 \\
\hline 5 & C-17 & 21 through 25 \\
\hline
\end{tabular}

Table 4.2 Aircraft and Missions Available

An example of an APOE-APOD pair to mission assignment is given in Table 4.3. 


\begin{tabular}{|l|l|l|}
\hline Pair Number & APOE-APOD Pair & Assigned Missions \\
\hline 0 & {$[$ KDOV, LTAG] } & $1,11,7,16,24$ \\
\hline 1 & {$[$ KDOV , OKBK] } & $2,6,21,12,3$ \\
\hline 2 & {$[$ KDMA, ETAR] } & $13,8,17,23$ \\
\hline 3 & {$[$ KBLV, RJBB] } & 22,18, \\
\hline
\end{tabular}

Table 4.3 APOE-APOD Pairs and Assigned Missions

This arrangement can be represented as follows:

$(0,(1,11,7,16,24), 1,(2,6,21,12,3), 2,(13,8,17,23), 3,(22,18))$,

where APOE-APOD pair numbers are followed by an array of the ordered mission assignments to the APOE-APOD pair. In this example, there is undelivered cargo for the pair KDOV to OKBK (pair 1). Additionally, there are late deliveries of requirements for the pair KDMA-ETAR (pair 2).

The NMI neighborhood first determines the missions available for insert. The unused missions are: $(4,5,9,10,14,15,19,20,25)$. From the set of unused missions, at most one mission number for each aircraft is added to the insert list. Thus, in this example, the set of possible insert missions are $(4,9,14,19,25)$. Unused missions are then sorted according to earliest time available under each aircraft's current assignment scheme.

The set of APOE-APOD pairs considered are restricted to those pairs with undelivered cargo and/or late cargo. Undelivered cargo is targeted first and pairs are sorted for insert based on the total quantity of undelivered cargo. Pairs with 
late cargo are considered next and ordered according to the product of the tonnage and amount of lateness.

Once the insert missions and pairs are identified, the set of insert locations are determined. Returning to the example, there are only 6 possible insert points for unused mission 19 into pair 1 (KDOV to OKBK). Using the candidate list structure described above, the possible insert points are identified and added to the candidate list. Unless all missions for the APOE-APOD pair under consideration possess a last ALD close to the late/undelivered requirement ALD or carry some portion of the requirement, the number of insert points will be less than the 6 possible insert points. Typically requirement ALDs are spread over time for realistic TPFDDs and the number of filtered insert points will be significantly less.

\subsubsection{Between Pair Swap Move Neighborhood (BPS)}

BPS moves swap missions between two different APOE-APOD pairs. Allowable swaps are those that swap two different types of aircraft or two different aircraft. Swapping missions assigned to the same aircraft would be a null move, i.e., illogical and wasteful of computational effort. In an effort to target poor mission assignments, a dynamic functional candidate list is created to sort the APOE-APOD pairs and the missions within those pairs for consideration. Swaps are then investigated based upon the sorted pairs and missions within the pairs.

Under the functional candidate list scheme, mission quality is assessed using the mission attributes of late requirements and unused aircraft capacity. 
The late requirement value is the product of the quantity late (tons) and the amount late (days). Unused aircraft capacity is the difference between the aircraft ACL and the current payload. A mission's quality value is the sum of these two measures. Missions are sorted in descending order within the APOE-APOD pair according to this measure. APOE-APOD pair quality is the sum of the assigned mission values and any undelivered cargo. These three measures are readily obtained and provide a sufficient means to evaluate current mission assignments. Late requirements and undelivered requirements provide a quick assessment of aircraft assignments. Unused aircraft capacity measures not only how well aircraft are loaded but also (indirectly) the current missions assignment's impact on MOG and UTE constraints - small loads mean more aircraft missions and hence a greater strain upon the airlift network.

Continuing with the example, for the current pair to mission assignment, $(0,(1,11,7,16,24), 1,(2,6,21,12,3), 2,(13,8,17,23), 3,(22,18))$, there are 8 possible swap moves between APOE-APOD pairs 2 and 3. Under the BPS move neighborhood however, only 6 are allowed-swaps $(22,23)$ and $(17,18)$ are not allowed since they are missions for the same aircraft. The order in which these swaps are considered is based upon the candidate list structure described above.

Subsequent iterations begin at the last BPS swap location to ensure that other regions of the solution space are investigated. This creates a rotating list of swaps that moves through the APOE-APOD pairs sequentially, starting over whenever all swaps for the last two APOE-APOD pairs are exhausted. 


\subsubsection{Within Pair Insert Move Neighborhood (WPI)}

WPI moves seek improvements in mission assignments within APOEAPOD pairs. Thus WPI moves are intensifying moves. Under the WPI neighborhood, a mission assigned to a given APOE-APOD pair can be inserted in a different position within the current set of missions assigned to the pair. Candidate APOE-APOD pairs for WPI moves are sorted by contribution to the objective function. In this way, APOE-APOD pairs that contribute most to the objective are investigated first. The mission assignment/scheduler heuristic attempts to assign missions to requirements based upon the requirement RDD, mission ordering, and aircraft compatibility with the requirement. Changing the order of missions within an APOE-APOD pair can lead to better load efficiencies and improvements in temporally based objective values (working MOG, fuel MOG, cargo lateness, and UTE rate).

As an example, consider the following mission assignment for an APOEAPOD pair:

$(252,140,147,154,161,168,0,163,170,289,296,303,310,387)$.

The current APOE arrival times for the missions are summarized in Table 4.4. 


\begin{tabular}{|c|c|}
\hline Mission Number & APOE Arrival Time (days) \\
\hline 252 & 0.17708 \\
\hline 0 & 0.18167 \\
\hline 140 & 0.22324 \\
\hline 147 & 0.22324 \\
\hline 154 & 0.22324 \\
\hline 161 & 0.22324 \\
\hline 168 & 0.22324 \\
\hline 387 & 11.09375 \\
\hline 163 & 11.12500 \\
\hline 170 & 11.12500 \\
\hline 289 & 11.17708 \\
\hline 296 & 11.17708 \\
\hline 303 & 11.17708 \\
\hline 310 & 11.17780 \\
\hline & \\
\hline
\end{tabular}

Table 4.4 APOE Arrival Times

Missions are sorted in the table by arrival time at the APOE. Observe the position of Mission 0 in the mission assignment array and in Table 4.4. The temporal ordering of missions is not (necessarily) the same as the mission assignment ordering. Under the mission assignment/scheduler heuristic, aircraft missions are assigned based upon several factors that may preclude missions from 
being assigned strictly according to mission assignment order. These factors are: aircraft availability time (due to prior missions), aircraft compatibility with the current controlling requirement, and aircraft compatibility with other requirements available at the APOE on the current controlling requirement's ALD.

Because of this phenomenon, it is difficult to determine where good insert points lie. Ideally all possible inserts are considered, however, for typical instances of the SAP, the number of inserts can be prohibitively large. As a compromise, the insert points for each mission within an APOE-APOD pair are restricted to be within an insert move distance that is based upon instance size.

The number of missions per pair in the initial solution is used as a rough measure of instance size. Two measures are used to determine the insert move distance - the average number of missions per pair (AM/P) and the median number of missions per pair $(\mathrm{MM} / \mathrm{P})$. These measures provide an estimate of the typical number of missions per APOE-APOD pair. The insert move distance is set to the maximum of the default move distance (set to 5 in this implementation), $\mathrm{AM} / \mathrm{P}$ and $\mathrm{MM} / \mathrm{P}$. In this way the insert move distance is tied to instance size and a reasonable portion of the possible inserts are investigated. WMI inserts are all possible inserts within insert move distance to the left and right of the insert mission.

Returning to the example, assume that the insert move distance is 5 . The possible WPI insert points for Mission 0 are shown in Figure 4.4 below: 


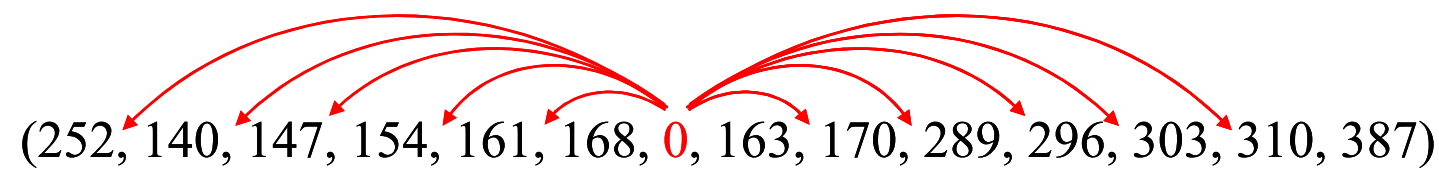

Figure 4.4 WPI Insert Points for Mission 0

\subsubsection{Between Pair Insert Move Neighborhood (BPI)}

The BPI move neighborhood removes an assigned mission from one APOE-APOD pair and inserts it into a different APOE-APOD pair. BPI moves change the cardinality of the current mission assignment structure and therefore is a diversifying move. Because BPI reduces the number of missions assigned to an APOE-APOD pair, it likely results in a large undelivered penalty. Thus, BPI is used sparingly as a temporary infeasible diversifier that may also add heretofore unavailable trajectories to the search. Its use is curtailed to conditions where diversification is appropriate. Diversification conditions are described in the Move Manager section below (Section 4.10).

The dynamic functional candidate list described for BPS moves in Section 4.6.3 is used to determine the ordering of APOE-APOD pairs and their mission assignments. However, for the BPI neighborhood, ordering of candidate pairs and missions for consideration is in ascending order. This targets poor pair assignments and identifies inserts from other missions that may improve the objective. BPI moves that render an APOE-APOD pair devoid of missions are not allowed. 


\subsubsection{Within Mission Arc Swap Move Neighborhood (WMAS)}

WMAS moves target improvements in working MOG and fuel MOG at the mission level. WMAS candidates are those missions in the current solution that contribute to working MOG and/or fuel MOG violations. Recall each Arc has a start base and end base that are connected by a route segment. The route segment consists of the waypoints and bases the aircraft passes through when traveling from the start base to the end base. In the case of a direct flight, no intermediate bases are traversed and swapping the associated Arc would have no effect. Thus swapping an $\operatorname{Arc}$ in the current routing scheme can change the set of bases that the aircraft passes through and thus reduce congestion at intermediate bases.

For the special case of APOE-APOD Arcs, a new Arc can have a smaller maximum cargo than the incumbent Arc. In this case cargo must be removed to meet the new maximum cargo limit. For WMAS, late requirements are first considered for ejection then requirements are dropped based upon compatibility and RDD order until the maximum cargo limit is met. Ejected cargo is then added to the undelivered cargo list.

In the case where cargo is ejected as a result of a WMAS move, there is an opportunity to employ powerful compound moves called ejection chains (Glover and Laguna, 1997). A route change that forces cargo to be ejected causes a change in the assignment of cargo as well as the routing of the mission. Ejected cargo as part of a WMAS move immediately invokes the LR move neighborhood to determine the best mission to load some or all of the undelivered cargo. The 
value of the WMAS move then is the result of this compound move. This ejection chain feature is incorporated into SAP-TS and can be invoked throughout the search or as a result of conditions encountered during the search.

\subsubsection{Load Reallocation Move Neighborhood (LR)}

The LR move neighborhood strives to increase fill efficiencies and reduce the total number of missions required. LR moves target undelivered cargo and those missions with payloads below the aircraft's trivial load threshold. If there is undelivered cargo, LR moves attempt to load undelivered cargo on existing missions. The LR move neighborhood assigns cargo from aircraft with trivial loads to aircraft with excess cargo capacity (percent capacity remaining $>0$ ).

Spatial and temporal restrictions limit candidate missions for the LR move neighborhood. Candidate "onto" missions must service the same APOE-APOD pair as that of the undelivered requirement or the trivial load mission. Additionally, "onto" missions must fall within the LR move distance. The LR move distance is a temporal restriction. An "onto" mission is a candidate if the difference between the new requirement ALD and the "onto" mission's APOE arrive time is less than the LR move distance. A default move distance of 3 days was selected because typical mission cycle times are less than three days and delaying a mission more than this amount is usually more costly than just creating a new mission.

Undelivered cargo moves are considered first and then moves for missions with less than trivial loads. If all requirements of a trivial load mission are removed, the mission is deleted from the solution. 


\subsubsection{Recover to APOE Arc Insert Move Neighborhood (RAI)}

RAI moves are specialized moves designed to reduce requirement lateness by altering an aircraft's routing. Specifically, RAI moves delete a REC to HB Arc and insert a REC to APOE Arc based upon the APOE visited by the aircraft's next mission. In this way the time from REC base to HB and from HB to APOE is replaced with the (shorter) REC to APOE time. Figure 4.5 illustrates the RAI move.

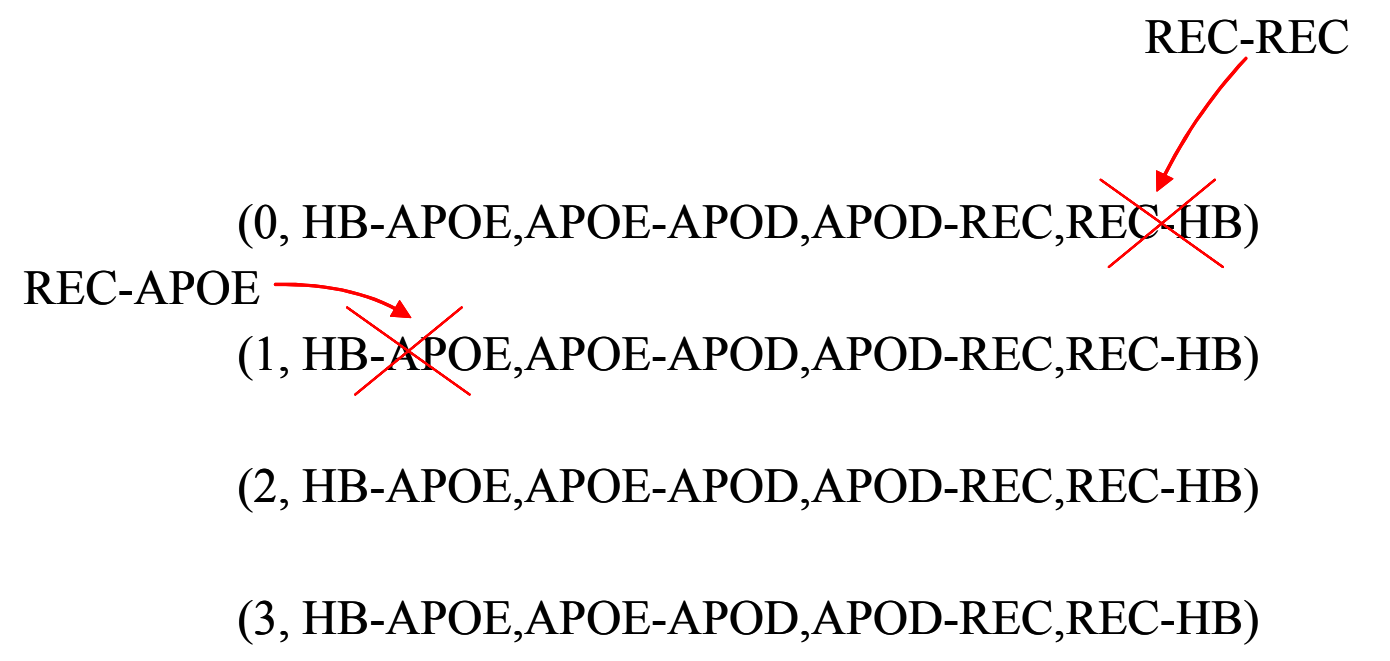

Figure 4.5 RAI Move

RAI moves are constrained by the return to base (RTB) time. The RTB constraint is an aircraft unit attribute. Aircraft assigned to the same unit share the same RTB time. In practice, a new mission cannot be planned if the time since the aircraft last visited its HB exceeds the RTB time. If the RTB is exceeded, the aircraft must return to its HB before a new mission can be planned. In SAP-TS, RTB time violations are determined as follows: the last aircraft HB departure 
time is determined. Then, for the mission in which a REC to HB arc is replaced with a REC to REC arc, the time available at the REC base (arrival time at the REC base plus en route time) is determined. If the difference between time available at the recovery base and the last HB departure time is less than the RTB time, the RAI move is permissible. As an example, suppose that an aircraft (a C5A) has the following set of missions assigned under the current solution:

$$
(0,2,53,170,309)(1,2,72,176,541)(2,2,43,162,179) \text {. }
$$

Further, suppose that we desire to invoke an RAI move that involves missions 0 and 1 . Let the RTB time for a C-5A be 3 days and the en route time 3.25 hours (0.14 days). Let the depart time from the HB for mission 0 be at time 0- the last time the aircraft was at its HB. Arc object 170 is the APOD to REC arc for mission 0. Suppose that arrival at the REC base is at 1.61 days. Thus the aircraft is available at the REC base at $1.61+0.14$ or 1.75 days. Since the difference between the time available at the REC base (1.75 days) and the time last at its HB (0.00 days) is less than the RTB time of 3 days, the RAI mission may be planned.

A candidate list of RAI missions is constructed as follows. Aircraft with missions containing late requirements are identified and sorted by their lateness contribution. Then, for each of these aircraft, missions are identified that may feasibly move directly to another APOE.

\subsubsection{Impose Time Delay Move Neighborhood (ITD)}

WMAS moves may not completely remove working MOG and fuel MOG violations. Violations that occur at endpoint bases are usually unaffected by 
WMAS moves since only intermediate bases are exchanged. Also, route segments frequently have some bases in common thus swapping an arc may not remove MOG violations for some intermediate bases.

ITD moves are invoked to temporally stagger aircraft through the network to alleviate congestion (working MOG and fuel MOG violations) and reduce the number of aircraft operating at any given time (UTE rate). Generally, working MOG violations accompany fuel MOG and UTE rate violations since working MOG violations indicate aircraft clustering. Thus, ITD moves that target working MOG violations often have the secondary effect of reducing fuel MOG and UTE rate violations as well. For this reason, ITD moves target in sequence working MOG, then fuel MOG and finally UTE rate violations. ITD move attributes consist of the mission number, the arc location at which the delay is imposed, and the amount of the delay (in days).

A specialized candidate list strategy is imposed to target missions that contribute to MOG violations (working and fuel) and UTE rate violations. For working and fuel MOG violations, bases are ordered by the respective total violation. Then, for each base, the missions contributing to the violation are identified and sorted by slack time. Slack time is defined as the difference between earliest RDD time for the requirements on the mission and mission arrival time at the APOD. The intent is to target missions that can be delayed without increasing lateness first. Sorted missions are then investigated in turn and the delay is computed. The delay for a mission violating either a working MOG or fuel MOG constraint is the maximum ground time (in days) divided by 
the base MOG. The maximum ground time is a user setting. The default setting of 5 hours exceeds the maximum ground time for all aircraft types (en route, onloading or offloading) in the current AMOS implementation. This delay has the effect of staggering aircraft through the constraining base sufficiently to reduce MOG violations without imposing too great a delay.

For UTE rate violations, the focus is on aircraft and not bases. Aircraft types are sorted for consideration using the aircraft type's UTE rate violation. Missions are then sorted by aircraft type and then, once again by slack (as defined above) within aircraft type. The amount of delay imposed is computed in the same manner as described above.

\subsection{TABU STRUCTURE}

Tabu restrictions are constraints imposed on allowable moves based upon move attributes. Tabu restrictions are used to prevent the reversal of recent moves and to allow the search to escape from local optima. The duration of tabu restrictions is the tabu tenure. Moves whose attributes are restricted under the current tabu list are considered tabu and are not allowed for the duration of tabu tenure iterations. There are two tabu lists implemented in SAP-TS to impose tabu restrictions. They mirror the dichotomy in the SAP-TS move neighborhoods - a tabu list for the mission assignment phase (assignmentTabuList) and a tabu list for the mission improvement phase (missionTabuList).

The tabu list for the mission assignment phase, assignmentTabuList, is invoked during the mission assignment phase of the search. The assignmentTabuList attributes consist of the APOE-APOD pair, mission number 
and the position of the mission in the current APOE-APOD pair mission assignments. For insert moves (NMI, BPI, and WMI), the position is the location from which the inserted mission came from. For swap moves (BPS), both missions are added to the tabu list along with their original position in the current APOE-APOD pair mission assignments. As an example, suppose that the current tabu tenure is 7 , the current iteration is 18 and the current APOE-APOD pair to mission assignment is:

$(0,(1,11,7,16,24), 1,(2,6,21,12,3), 2,(13,8,17,23), 3,(22,18))$.

If a BPS move is performed swapping mission 16 with 21 , any move that either moves mission 16 back to APOE-APOD pair 0 at position 3 OR moves mission 21 back to APOE-APOD pair 1 at position 2 before iteration 25 is considered tabu.

For an insert move, if mission 16 is inserted into APOE-APOD pair 2 at position 1 under a BPI move, a move that returns mission 16 to position 3 in APOE-APOD pair 0 before iteration 25 is considered tabu.

The tabu structure for the mission improvement phase, missionTabuList, is a list consisting of the move mission number and location within the mission array where the change occurred. Tabu active status duration (current iteration plus tabu tenure) is stored in an array of size 5 at the position where the change occurred. This array mirrors the mission array structure in a SAP-TS solution(mission node\#, arc\#, arc\#, arc\#, arc\#). Thus the first position corresponds to the mission node, the second to the first arc traversed in the route, the third to the second arc traversed in the route and so on until the last arc at the fifth and final 
position. The position in the array at which the tabu active status is stored is based upon the move neighborhood and where the change in the mission array occurs. An LR move changes the payload for two missions-from mission and to mission. Payload changes affect the mission node (position 0 in a mission array) therefore tabu active status for an LR move is stored in position 0. WMAS and RAI moves represent changes in the routing of a mission. Tabu active status for these moves is stored at the location corresponding to the $\operatorname{arc}(\mathrm{s})$ that was changed. ITD moves store tabu active status at the arc position at which the time delay was imposed. In this way, mission improvement phase move attributes that affect specific portions of the mission, payload or routing, are recorded to prevent reversion to recent solutions. Note that mission improvement phase tabu attributes are based upon the solution array and not the APOE-APOD pair to mission assignment used in the mission assignment phase above.

As an example, suppose that the following missions represent a portion of the current solution at iteration 10 (and that the tabu tenure is still 7):

$$
(0,2,53,170,309)(1,2,72,176,541)(2,2,43,162,179) \text {. }
$$

Suppose that the current move is a WMAS move for mission 1 that swaps arc 72 with arc 73 resulting in the new mission $(1,2,73,176,541)$. The tabu array corresponding to mission 1 would be set to $(0,0,17,0,0)$. Mission improvement moves that affect the arc at position 2 are tabu if the current iteration is not greater than 17. Suppose an LR move is applied to the current solution that removes some portion of the payload from mission 0 (from mission) and places it on mission 1 (to mission). Only the to mission (mission 2) tabu 
status is updated to preclude removal of cargo from a mission that just received additional cargo. This also permits consideration of the from mission payload in the next iteration if payload still remains. In this way trivial load missions can be quickly removed from the solution. Under this move, the tabu array for mission two would be set to $(17,0,0,0,0)$. Tabu active status for an ITD move is set at the arc location where the time delay is imposed.

RAI moves affect the tabu status of more than one mission. Continuing with the solution excerpt from above,

$$
(0,2,53,170,309)(1,2,72,176,541)(2,2,43,162,179) \ldots,
$$

suppose that an RAI move is imposed on missions 0 and 1 such that the last arc of mission 1 becomes a REC to REC arc and the first arc of mission 1 becomes a REC to APOE arc. For this move the tabu arrays of mission 0 and 1 become $(0$, $0,0,0,17)$ and $(0,17,0,0,0)$ respectively.

A tabu active move may be accepted if it meets some aspiration criterion. In SAP-TS, the aspiration criterion is met if the tabu move value is better than the global current best solution. In this case, the tabu status is overridden and the move is allowed.

\subsection{SAP TABU SEARCH ALGORITHM}

SAP-TS proceeds in turn through the following phases:

- Preprocessing

○ Read input files

○ Build objects/Initialize data

○ Construct arcs and aircraft nodes 
- Construct Initial Solution

- Evaluate Initial Solution

- Conduct Tabu Search

- Initialize Tabu Search Objects

○ Start search

○ Select Move Neighborhood Phase

○ Select Move Neighborhood

- Evaluate Moves

- Select Best Non-Tabu Move

- Perform selected move

○ Update Solution/Solution Value

- Elite List Iterations (Optional)

- Postprocessing

- Save solution and solution objects to file

○ Output solution

- Mission Summary Output

- Detailed Itinerary Output

Key tabu search parameters and default settings are summarized in Table 4.5 . 


\begin{tabular}{|c|c|c|}
\hline Parameter & Default Setting & Description \\
\hline NoChangeMovesTolerance & $\begin{array}{llll} & 0 & 0\end{array}$ & $\begin{array}{l}\text { \# no change value moves allowed } \\
\text { before diversifying }\end{array}$ \\
\hline UnimprovingMovesTolerance & 3 & $\begin{array}{l}\text { \# unimproving moves allowed before } \\
\text { diversifying }\end{array}$ \\
\hline SuperDiversifyTolerance & 10 & $\begin{array}{l}\text { \# small change moves allowed } \\
\text { before superdiversifying }\end{array}$ \\
\hline SolutionHistoryLength & 20 & $\begin{array}{l}\text { \# previous objectives to consider for } \\
\text { superdiverisfication }\end{array}$ \\
\hline ObjectiveTolerance & 0.01 & $\begin{array}{l}\text { tolerance for objective in } \\
\text { superdiversification count }\end{array}$ \\
\hline ChooseFirstImprovingMove & TRUE & $\begin{array}{l}\text { boolean for selecting the first } \\
\text { improving move }\end{array}$ \\
\hline MaxCompTime & 40 & $\begin{array}{l}\text { maximum search time (hours) alotted } \\
\text { for the search }\end{array}$ \\
\hline NumAircraftTrips & 12 & \# aircraft trips per aircraft \\
\hline NumRoutesToConsider & 2 & $\begin{array}{l}\text { maximum number of routes to create } \\
\text { for each arc }\end{array}$ \\
\hline MsnAssignmentPhase & TRUE & $\begin{array}{l}\text { boolean that sets mission assignment } \\
\text { phase status }\end{array}$ \\
\hline MsnImprovementPhase & FALSE & $\begin{array}{l}\text { boolean that sets mission } \\
\text { improvement phase status }\end{array}$ \\
\hline MsnAssignmentNBHSizeLimit & 100 & $\begin{array}{l}\text { mission Assignment Phase } \\
\text { maximum NBH size }\end{array}$ \\
\hline msnImprovementNBHSizeLimit & 800 & $\begin{array}{l}\text { mission Improvement Phase } \\
\text { maximum NBH size }\end{array}$ \\
\hline WorkingMOGTolerance & 0 & $\begin{array}{l}\text { threshold below which working } \\
\text { MOG feasible }\end{array}$ \\
\hline FuelMOGTolerance & 0 & $\begin{array}{l}\text { threshold below which fuel MOG } \\
\text { feasible }\end{array}$ \\
\hline UteRateTolerance & 0 & $\begin{array}{l}\text { threshold below which UTE rate } \\
\text { feasible }\end{array}$ \\
\hline NumberOfIterations & 400 & \# tabu search iterations \\
\hline Tenure & 7 & duration (iterations) of tabu attribute \\
\hline EliteListSize & 3 & number of elite solutions to retain \\
\hline ObjFnWeights & $\{1,1,1,1,1,1,1,1,1,1\}$ & $\begin{array}{l}\text { weights for changing priority of } \\
\text { objective values }\end{array}$ \\
\hline loadReallocationMoveDistance & 3 & $\begin{array}{l}\text { temporal distance (days) for LR } \\
\text { moves }\end{array}$ \\
\hline InsertDistance & 5 & $\begin{array}{l}\text { maximum insert distance (left \& } \\
\text { right) for WPI moves }\end{array}$ \\
\hline
\end{tabular}

Table 4.5 Tabu Search Parameters 
Tabu search parameters control the course of the search through a series of counters, tabu search listeners and thresholds. Details on how these parameters are employed are described in the Move Manager section (Section 4.10).

There are several settings related to calculations in an instance of the SAP. These parameters affect calculations concerning timings, loading, and fuel calculations. Additionally, the search space size is controlled by airlift network size (numRoutesToConsider) and the allocation of aircraft missions (numAircraftTrips). These problem instance parameters are summarized in Table 4.6 below.

\begin{tabular}{|c|c|c|}
\hline Parameter & Default Setting & Description \\
\hline payLoadThreshold & 0.05 & $\begin{array}{c}\text { percentage below which } \\
\text { payload calculations will stop }\end{array}$ \\
\hline Precision & 0.001 & $\begin{array}{c}\text { precision level used in } \\
\text { calculations }\end{array}$ \\
\hline appLandTime & 15 & $\begin{array}{c}\text { standard approach \& land time } \\
\text { (minutes) }\end{array}$ \\
\hline PaxWeight & 400 & $\begin{array}{c}\text { standard passenger weight } \\
\text { (lbs) }\end{array}$ \\
\hline ZeroTemp & 18.3333 & $\begin{array}{c}\text { temperature (0C) at zero } \\
\text { alttitude }\end{array}$ \\
\hline mogInterval & 0.25 & $\begin{array}{c}\text { interval (hours) for working } \\
\text { MOG calculations }\end{array}$ \\
\hline Horizon & 40 & planning horizon (days) \\
\hline poundsPerGallonOfFuel & 6.8 & $\begin{array}{c}\text { conversion factor for fuel } \\
\text { calculations }\end{array}$ \\
\hline maxGroundTime & 5 & $\begin{array}{c}\text { maximum ground time (hours) } \\
\text { across aircraft types }\end{array}$ \\
\hline numAircraftTrips & 12 & \# aircraft trips per aircraft \\
\hline numRoutesToConsider & 2 & $\begin{array}{c}\text { maximum number of routes to } \\
\text { create for each arc }\end{array}$ \\
\hline
\end{tabular}

Table 4.6 Problem Instance Parameters 
The next several sections describe details of major components of the SAP-TS algorithm.

\subsection{MISSION ASSIGNMENT/SCHEDULER HEURISTIC}

The mission assignment/scheduler heuristic is similar to the method used to construct the initial solution. The major difference is that the mission assignment/scheduler heuristic obtains a solution using a given ordered assignment of aircraft missions to the APOE-APOD pairs. Requirements are sorted by earliest ALD and then by RDD. The heuristic attempts to sequentially load the next available requirement on the next available aircraft assigned to the APOE-APOD pair. If the current aircraft is not compatible with the current requirement or not completely filled by the current requirement, other requirements available at the same APOE-APOD pair and on the same day are considered for loading. In this way, a solution is constructed based upon the ordered assignment of available missions at APOE-APOD pairs and prioritized requirements. Pseudocode for the mission assignment/scheduler heuristic is presented in Figure 4.6.

Two additional measures drive the mission assignment/scheduler heuristic to obtain solutions that are closer to feasibility than the initial solution. The first is associated with how arcs are selected during mission construction and the second is associated with mission timings along the arcs. 


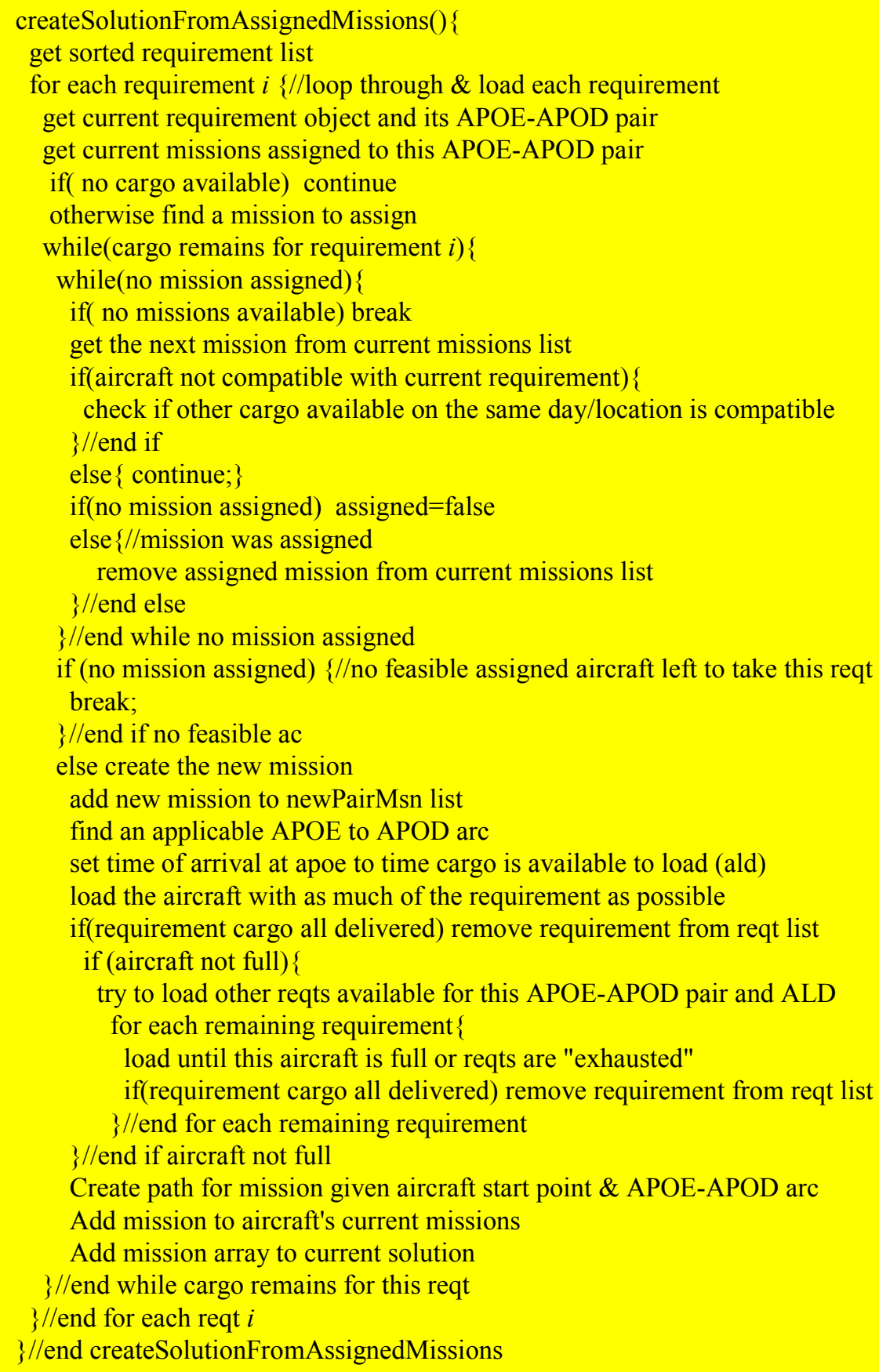

\section{Figure 4.6 Mission Assignment/Scheduler Heuristic Pseudo Code}


Arcs are selected using a rotating list for each set of route segments connecting two endpoints. For example, suppose that the current mission for a C5A (aircraft type MIL) requires a route from KDOV to OKBK- the APOE to APOD arc. The set of arcs connecting KDOV to OKBK under the current route plan are shown in Table 4.7.

\begin{tabular}{|c|c|c|c|c|}
\hline Arc\# & Seg\# & AcTypes & $\begin{array}{c}\text { C-5A } \\
\text { Max Cargo (tons) }\end{array}$ & \# En route Bases \\
\hline 52 & 747 & CIV & N/A & 2 \\
\hline 53 & 748 & CIV & N/A & 2 \\
\hline 54 & 734 & MIL & 83.98 & 1 \\
\hline 55 & 735 & MIL & 84.40 & 1 \\
\hline 56 & 736 & MIL & 77.31 & 1 \\
\hline 57 & 737 & MIL & 79.42 & 1 \\
\hline 58 & 738 & MIL & 88.07 & 1 \\
\hline 59 & 739 & MIL & 87.06 & 1 \\
\hline 60 & 740 & MIL & 85.31 & 2 \\
\hline 61 & 741 & MIL & 85.72 & 2 \\
\hline 62 & 742 & MIL & 78.82 & 2 \\
\hline 63 & 743 & MIL & 80.93 & 2 \\
\hline 64 & 744 & MIL & 88.59 & 2 \\
\hline 65 & 745 & MIL & 88.07 & 3 \\
\hline 66 & 746 & MIL & 109.94 & \\
\hline
\end{tabular}

Table 4.7 Arcs Connecting KDOV to OKBK

Since the aircraft is a military aircraft, only arcs 54 to 66 apply. As this is an APOE-APOD route, we desire the arc with the largest maximum cargo so that payload is not constrained by the route. The maximum cargo range is [77.31, 109.94] and the average is 85.97 tons. Note that the arc with the greatest maximum cargo (arc 66) also uses the most en route bases - three in all. Thus the increase in possible payload comes at the cost of additional strain on the airlift 
network. Thus if APOE to APOD arcs were selected solely on the basis of arc maximum cargo, bottlenecks would develop reducing throughput. For this reason arcs are selected from the possible set on a rotating basis. If the current mission is assigned arc 66 the next mission (with a KDOV to OKBK arc) will be assigned arc 54 and so on.

The second feature employed in the mission assignment/scheduler heuristic is a mechanism to stagger aircraft flow through the airlift network. As arcs are selected, a running tally is kept of the depart times from the arc's starting base. As an arc is employed in subsequent missions, a check is made to see if "too many" aircraft are starting down the arc at the "same time." "Too many" aircraft is defined as more aircraft moving down the arc than the minimum wide body aircraft working MOG (WBWMOG) for all the bases in the arc. WBWMOG is selected because wide body aircraft slots at airfields are generally more limited than narrow body slots. Additionally, narrow body aircraft can often use narrow and wide body aircraft slots interchangeably. Aircraft are considered to be moving down the arc at the "same time" if they depart from the arc start base within the user defined maximum ground time (default is 5 hours) of each other. Aircraft missions meeting this temporal criterion are counted and the total is compared to the WBWMOG. If the WBWMOG is exceeded, the current mission's departure time from the arc start base is delayed by the ratio maximum ground time/WBWMOG. 


\subsection{MOVE MANAGER}

The move manager is the heart of SAP-TS and determines the appropriate phase, search context and move neighborhoods to apply to the current solution. Move neighborhoods are selected dynamically based upon current solution objective values and the search history.

SAP-TS alternates between neighborhood phasesmissionAssignmentPhase (MAP) and the missionImprovementPhase (MIP). Performing moves in the MAP are more expensive than MIP moves. For each MAP move considered, the mission assignment/scheduler heuristic is called. As problem size increases so does the time required to execute a call to the mission assignment/scheduler heuristic. MIP moves employ change calculations updating only the affected missions and are therefore much faster to evaluate. This is why the MAP maximum neighborhood size is set to a smaller value than the MIP maximum neighborhood size. Several mechanisms are employed to ensure that the neighborhood phases alternate periodically.

Figure 4.7 illustrates how the search phase and search context is determined. The search phase will remain the same if a new best or improving move is made. If either the superdiversification, unimproving move or no change move thresholds are exceeded, the search phase is changed. Once the search phase is determined, the context of search (superdiversify, intensify or diversify) is determined. 


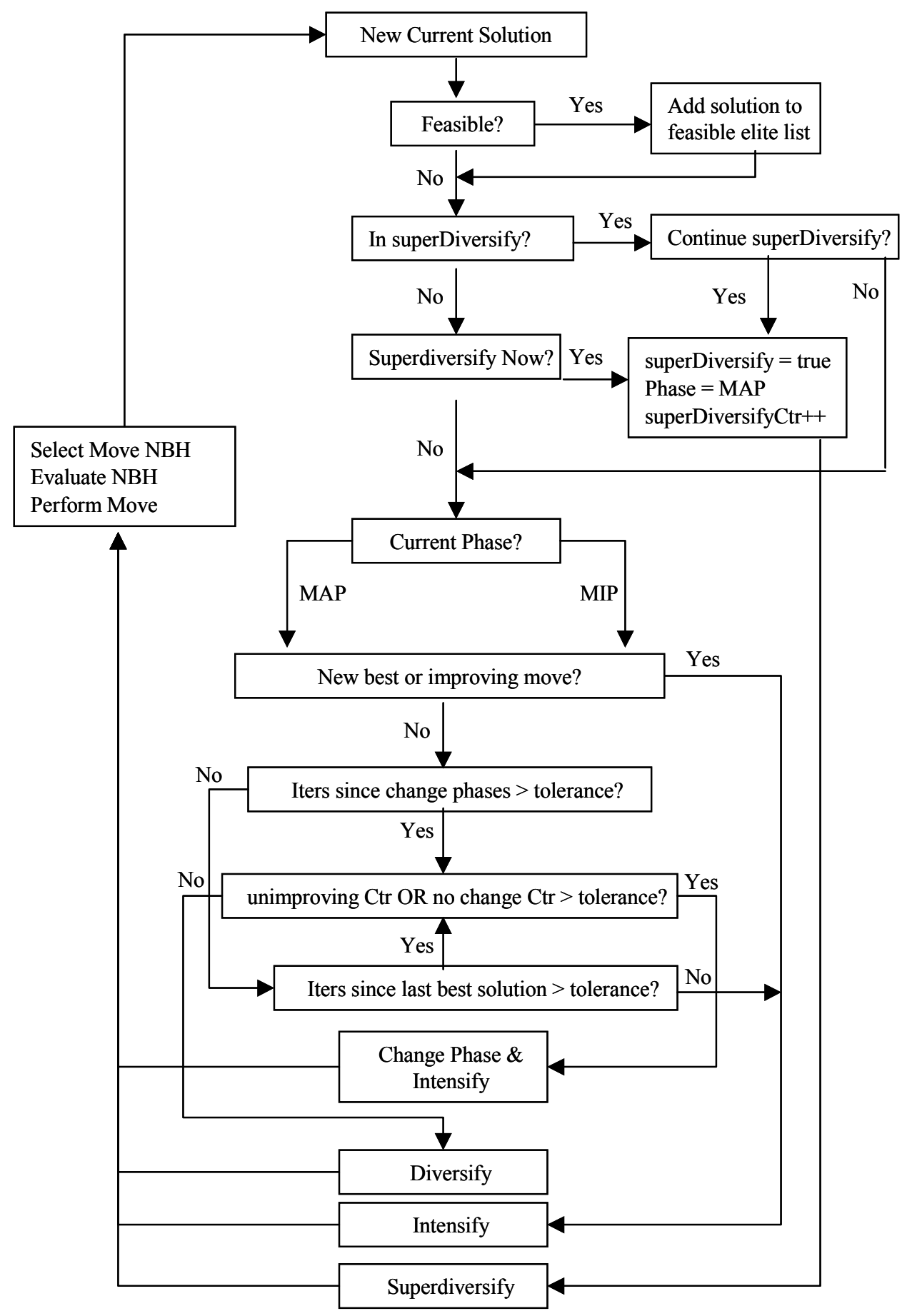

Figure 4.7 Determination of Neighborhood Phase and Search Context 
Superdiversification conditions are met if, within the last solutionHistoryLength iterations, the number of objective function values that are within the objectiveTolerance of the current objective exceeds the superDiversifyTolerance. If superdiversification is called for, the phase is set to MAP and the neighborhood to BPI. Once superdiversification conditions are met, a total of numberSuperDiversifyMoves superdiversification iterations are executed. Default settings for each of these parameters are listed in Table 4.5.

If superdiversification criteria are not met, additional checks are performed to determine if the search context should be intensify or diversify. As shown in Figure 4.7, new best or improving moves invoke intensification as the search context. Diversification is called for if either the threshold for the number of iterations since phases were changed or the threshold for the number of iterations since the best solution is exceeded. If both the unimproving move and no change move thresholds are not exceeded, diversification becomes the search context. Otherwise the phase is changed and the search context becomes intensification.

Once the search phase and context are specified, the appropriate move neighborhood is selected. A given neighborhood phase restricts the possible move neighborhoods to the four neighborhoods associated with the phase. The search context also impacts upon the neighborhood selected. If the context is superdiversify, BPI becomes the active neighborhood. Intensification implies that the current neighborhood is finding good solutions and therefore the current neighborhood is maintained. In the special case where the phase changed and 
intensify is the context, the neighborhood is selected based upon the current objective. Neighborhood selections under this situation are summarized in Table 4.8 .

\begin{tabular}{|l|l|l|}
\hline Phase & Condition & Neighborhood \\
\hline \multirow{3}{*}{ Mission Assignment } & Undelivered/Late Cargo & NMI or BPS \\
\cline { 2 - 3 } & Infeasible & BPS or WMI \\
\hline \multirow{2}{*}{ Mission Improvement } & Undelivered/Late Cargo & LR or RAI \\
\cline { 2 - 3 } & Infeasible & WMAS or ITD \\
\hline
\end{tabular}

Table 4.8 Neighborhood Selection After Phase Change

Diversification implies that the current neighborhood should be changed. The new neighborhood is determined based upon the phase, the previous neighborhood and the current objective. For the MAP, if the current objective has undelivered or late cargo the selected neighborhood alternates between NMI \& BPS. Otherwise, the selected neighborhood alternates between BPS and WMI. For the MIP, the selected neighborhood alternates between LR and RAI if there is undelivered or late cargo. Otherwise, the selected neighborhood alternates between WMAS and ITD.

\subsection{AN ITERATION IN THE SAP-TS ALGORITHM}

At the start of the search, the phase is set to MAP, search context to intensify and the neighborhood to BPS. An iteration in SAP-TS begins with a new current solution. As described in the previous section, search phase, context 
and neighborhood are chosen based upon a series of criteria. Once the neighborhood is identified, the set of possible moves given the neighborhood and the current solution are evaluated. The best non-tabu move is selected. The tabu active status of tabu moves that meet the aspiration criterion (move value is a new best solution value) are overridden.

The selected move is performed and the solution is updated to reflect the change. If the move is feasible, it is inserted into the feasible elite list. If the move is an unimproving move, the tabu tenure is incremented by one and the unimproving move counter is incremented. If the move yields no change in the objective function value, the no change in value counter is incremented. If the move is a new best or improving move, the unimproving and no change in value counters are reset to zero. A new best solution causes the tabu tenure to be reset to the default tenure. Additionally, the new best solution is added to the elite list.

The move manager is then invoked to determine the appropriate phase, context and neighborhood to employ based upon the new solution and the search history and the process repeats until either the maximum number of iterations have been performed or the maximum search time has elapsed.

\subsection{SUMMARY}

This chapter described the SAP-TS algorithm. SAP-TS partitions the search into two alternating phases: mission improvement phase and the mission assignment phase. Within each phase a set of specialized neighborhoods is employed. For the mission assignment phase, the NMI, BPS, WPI, and BPI move neighborhoods seek to improve the assignment of missions to APOE-APOD 
pairs. Moves are sent to the mission assignment/scheduler heuristic to obtain a new assignment. The mission improvement phase neighborhoods, LR, WMAS, ITD and RAI, are specialized neighborhoods that attempt to improve missions under the current mission assignment. The SAP-TS move manager uses a series of counters and search history to dynamically determine the appropriate phase, context and neighborhood at each iteration.

The next chapter describes the SAP problem instances employed to validate the efficacy of the SAP-TS algorithm. 


\section{Chapter 5: An Application of the SAP Tabu Search Algorithm}

This chapter provides an example application of the SAP Tabu Search algorithm (SAP-TS) to the Strategic Airlift Problem. Results are reported for application of SAP-TS to five SAP scenarios. SAP-TS results are then compared to AMOS results.

\subsection{SAP PROBLEM INSTANCES}

SAP-TS was applied to a set of five different scenarios of the Strategic Airlift Problem. These scenarios represent operationally realistic problem instances of the SAP and provide a sound basis to investigate the efficacy of SAPTS. A few of the scenarios were derived from AMC studies loosely based upon the deployment of an Army Stryker Brigade.

Each scenario was adjusted to emphasize different aspects typical of SAP instances. In particular, feasibility (working MOG, fuel MOG and UTE rate constraints) and problem size (number of requirements, number of unique APOEAPOD pairs and number of aircraft) elements were varied to provide a robust assessment of SAP-TS.

To discern between different scenarios, we employ the following elements to capture key problem characteristics: the number of aircraft, number of unique APOE-APOD pairs, total cargo (tons), total number of personnel and the feasibility constraints emphasized. Scenarios and their key features are summarized in Table 5.1 . 


\begin{tabular}{|l|c|c|c|c|c|}
\hline \multirow{2}{*}{ Scenario } & \multicolumn{4}{|c|}{ Problem Parameters } & \\
\cline { 2 - 6 } & $\begin{array}{c}\text { Number of } \\
\text { Aircraft }\end{array}$ & $\begin{array}{c}\text { Number of Unique } \\
\text { APOE-APOD } \\
\text { Pairs }\end{array}$ & $\begin{array}{c}\text { Total Cargo } \\
\text { (tons) }\end{array}$ & $\begin{array}{c}\text { Total } \\
\text { PAX }\end{array}$ & $\begin{array}{c}\text { Scenario } \\
\text { Emphasis }\end{array}$ \\
\hline Scenario 1 & 297 & 56 & 7647 & 14769 & (UTE/LATE) \\
\hline Scenario 2 & 124 & 45 & 15482 & 10576 & (LATE/MOG/FUEL) \\
\hline Scenario 3 & 297 & 95 & 13777 & 16683 & (MOG) \\
\hline Scenario 4 & 165 & 12 & 7647 & 14769 & (LATE) \\
\hline Scenario 5 & 70 & 12 & 3786 & 9769 & (Typical) \\
\hline
\end{tabular}

Table 5.1 Scenario Characteristics

Several measures were used to compare SAP-TS with AMOS. First, the SAP-TS objective function was used to provide a wholistic measure of SAP-TS results against AMOS results. Second, the makespan or closure time for the respective solutions was computed to measure the time required for all requirements to reach the respective APODs under the two schemes. Finally, a comparison of the daily cumulative tonnage closed was tabulated to determine how well required delivery times were met throughout the deployment.

\subsection{AN EXAmple Strategic Airlift Problem}

The smallest problem instance, Scenario 5, was selected to illustrate SAPTS. Scenario 5 details and the results associated with SAP-TS and AMOS are now presented. 


\subsubsection{Scenario 5 Details}

This particular deployment entails movement of equipment and personnel from five different APOEs in CONUS to four APODs in three different theatersSouthwest Asia, Europe and the Pacific.

The aircraft, home base and time available for each unit involved in the deployment are shown in Table 5.2. Multiple entries for a particular unit indicate that additional aircraft become available at later times in the deployment horizon. A total of 70 aircraft are available during the course of the deployment.

\begin{tabular}{|c|c|c|c|c|c|}
\hline Unit Name & $\begin{array}{c}\text { Type } \\
\text { Aircraft }\end{array}$ & $\begin{array}{l}\text { Home } \\
\text { Base }\end{array}$ & $\begin{array}{l}\text { RTB Time } \\
\text { (days) }\end{array}$ & $\begin{array}{c}\text { Day } \\
\text { Available }\end{array}$ & Qty \\
\hline \multirow[b]{2}{*}{ KBOS WBP Unit } & \multirow[b]{2}{*}{ WBP } & \multirow[b]{2}{*}{ KBOS } & \multirow[b]{2}{*}{10} & 0 & 5 \\
\hline & & & & 20 & 5 \\
\hline \multirow[b]{2}{*}{ KBOS NBP Unit } & \multirow[b]{2}{*}{ NBP } & \multirow[b]{2}{*}{ KBOS } & \multirow[b]{2}{*}{10} & 0 & 5 \\
\hline & & & & 20 & 5 \\
\hline \multirow[b]{3}{*}{ KBOS WBC Unit } & \multirow[b]{3}{*}{ WBC } & \multirow[b]{3}{*}{ KBOS } & \multirow[b]{3}{*}{10} & 0 & 5 \\
\hline & & & & 6 & 5 \\
\hline & & & & 20 & 5 \\
\hline \multirow[b]{3}{*}{ KDOV C-5A Unit } & \multirow[b]{3}{*}{ C-5A } & \multirow[b]{3}{*}{ KDOV } & \multirow[b]{3}{*}{10} & 0 & 10 \\
\hline & & & & 8 & 5 \\
\hline & & & & 20 & 5 \\
\hline ETAR C-17 Unit & $\mathrm{C}-17$ & ETAR & 10 & 0 & 5 \\
\hline KCHS C-17 Unit & $\mathrm{C}-17$ & KCHS & 10 & 0 & 5 \\
\hline PHIK C-17 Unit & $\mathrm{C}-17$ & PHIK & 10 & 0 & 5 \\
\hline
\end{tabular}

Table 5.2 Example Problem Available Aircraft

Requirements for this problem are shown in Table 5.3. 


\begin{tabular}{|c|c|c|c|c|c|c|c|c|c|}
\hline $\begin{array}{l}\text { REQT } \\
\text { ID }\end{array}$ & COMMODITY & ALD & RDD & OUT & OVER & BULK & PAX & APOE & APOD \\
\hline 1 & CSS Other & 0 & 1 & 0 & 33.5 & 0 & 300 & KBLV & LTAG \\
\hline 2 & $\mathrm{AF} \overline{A c f t}$ & 0 & 1 & 33.2 & 0 & 23.5 & 0 & KBLV & LTAG \\
\hline 3 & AF Supt & 0 & 2 & 0 & 18.1 & 0 & 0 & KDOV & LTAG \\
\hline 4 & Type 3 & 1 & 3 & 0 & 0 & 155.4 & 604 & KDMA & OKBK \\
\hline 5 & Type 4 & 0 & 3 & 13 & 6 & 180 & 233 & KDOV & OKBK \\
\hline 6 & Army_Prepo & 0 & 3 & 0 & 30.9 & 0 & 5 & KDOV & OKBK \\
\hline 7 & Airborne & 0 & 3 & 0 & 0 & 200.6 & 0 & KDOV & OKBK \\
\hline 8 & Armor & 1 & 3 & 0 & 6.6 & 0 & 1700 & KDSM & LTAG \\
\hline 9 & Mech & 1 & 3 & 37.2 & 0 & 0 & 0 & KDYS & LTAG \\
\hline 10 & AF Supt & 1 & 3 & 0.5 & 11.2 & 45.5 & 28 & KBLV & LTAG \\
\hline 11 & CSS Other & 4 & 7 & 0 & 0 & 10.2 & 341 & KDMA & LTAG \\
\hline 12 & CSS_Other & 4 & 7 & 16.5 & 98.6 & 0 & 3 & KBLV & LTAG \\
\hline 13 & AF $\overline{A c f t}$ & 7 & 9 & 0 & 0 & 155.3 & 643 & KBLV & LTAG \\
\hline 14 & AF Supt & 7 & 9 & 22.3 & 0 & 0 & 0 & KDMA & LTAG \\
\hline 15 & Armor & 5 & 9 & 0 & 0 & 34.5 & 0 & KDOV & LTAG \\
\hline 16 & Mech & 5 & 9 & 0 & 88.8 & 0 & 442 & KBLV & LTAG \\
\hline 17 & Airmobile & 5 & 9 & 5.6 & 6.7 & 0 & 41 & KBLV & LTAG \\
\hline 18 & Cbac & 7 & 9 & 0 & 75.9 & 0 & 0 & KDMA & OKBK \\
\hline 19 & Infantry & 7 & 9 & 0 & 0 & 20.3 & 2 & KBLV & LTAG \\
\hline 20 & Armor Cav & 9 & 10 & 50.6 & 0 & 0 & 322 & KDOV & $\overline{\text { LTAG }}$ \\
\hline 21 & Type_13 & 7 & 11 & 0 & 0 & 90.4 & 4 & KDOV & LTAG \\
\hline 22 & NEW 1 & 7 & 12 & 0 & 90.4 & 0 & 0 & KBLV & LTAG \\
\hline 23 & CSS $\overline{M e d}$ & 7 & 12 & 0 & 0 & 0 & 398 & KDMA & ETAR \\
\hline 24 & CSS_Signal & 9 & 12 & 32 & 0 & 0 & 0 & KDMA & ETAR \\
\hline 25 & CSS Supt maint & 9 & 12 & 0 & 10.5 & 8.9 & 0 & KDOV & ETAR \\
\hline 26 & CSS Trans & 1 & 13 & 0 & 14.5 & 0 & 120 & KDMA & ETAR \\
\hline 27 & CSS Other & 9 & 16 & 90.3 & 0 & 1.2 & 0 & KDOV & ETAR \\
\hline 28 & $\mathrm{AF} \overline{\mathrm{Acft}}$ & 11 & 16 & 0 & 0 & 287.8 & 0 & KDOV & OKBK \\
\hline 29 & AF Supt & 11 & 16 & 0 & 6.7 & 0 & 7 & KDMA & LTAG \\
\hline 30 & Type 3 & 11 & 16 & 0 & 0 & 155.4 & 604 & KDOV & LTAG \\
\hline 31 & Type 4 & 11 & 16 & 1.2 & 33.6 & 80 & 233 & KBLV & LTAG \\
\hline 32 & Army Prepo & 14 & 21 & 0 & 33.6 & 122.3 & 0 & KBLV & LTAG \\
\hline 33 & Airborne & 9 & 22 & 0 & 0 & 0 & 12 & KDMA & OKBK \\
\hline 34 & Armor & 11 & 23 & 98.8 & 0 & 7.9 & 0 & KDOV & OKBK \\
\hline 35 & Mech & 22 & 32 & 0 & 14.2 & 0 & 133 & KDMA & OKBK \\
\hline 36 & AF Acft & 4 & 9 & 50.6 & 0 & 0 & 322 & KDOV & RJBB \\
\hline 37 & AF Supt & 12 & 21 & 0 & 0 & 90.4 & 4 & KDOV & RJBB \\
\hline 38 & Type 3 3 & 24 & 27 & 0 & 0 & 155.4 & 604 & KDMA & RJBB \\
\hline 39 & Type 4 & 4 & 9 & 23.3 & 0 & 180 & 233 & KDMA & RJBB \\
\hline 40 & Army Prepo & 15 & 21 & 6.5 & 6.7 & 5.6 & 123 & KBLV & RJBB \\
\hline 41 & Airborne & 24 & 25 & 64 & 0 & 0 & 89 & KDOV & RJBB \\
\hline 42 & Armor & 11 & 28 & 0 & 110.4 & 0 & 0 & KBLV & RJBB \\
\hline
\end{tabular}

Table 5.3 Example Problem Requirements 


\begin{tabular}{|l|l|l|l|l|l|l|l|l|l|}
\hline $\begin{array}{c}\text { REQT } \\
\text { ID }\end{array}$ & COMMODITY & ALD & RDD & OUT & OVER & BULK & PAX & APOE & APOD \\
\hline 43 & Mech & 14 & 29 & 0 & 0 & 0 & 678 & KDOV & RJBB \\
\hline 44 & Airmobile & 11 & 23 & 32 & 0 & 0 & 0 & KDMA & RJBB \\
\hline 45 & Cbac & 20 & 23 & 0 & 10.5 & 8.9 & 0 & KBLV & RJBB \\
\hline 46 & Infantry & 15 & 23 & 0 & 44.5 & 0 & 120 & KDMA & RJBB \\
\hline 47 & Armor_Cav & 16 & 23 & 90.3 & 0 & 6.2 & 0 & KDOV & RJBB \\
\hline 48 & Type_13 & 24 & 28 & 0 & 0 & 0 & 309 & KDOV & RJBB \\
\hline 49 & Navy_Marines & 21 & 23 & 18 & 0 & 0 & 0 & KBLV & RJBB \\
\hline
\end{tabular}

Table 5.3 (continued) Example Problem Requirements

Figure 5.1 graphically depicts the example problem requirements and aircraft available over time.

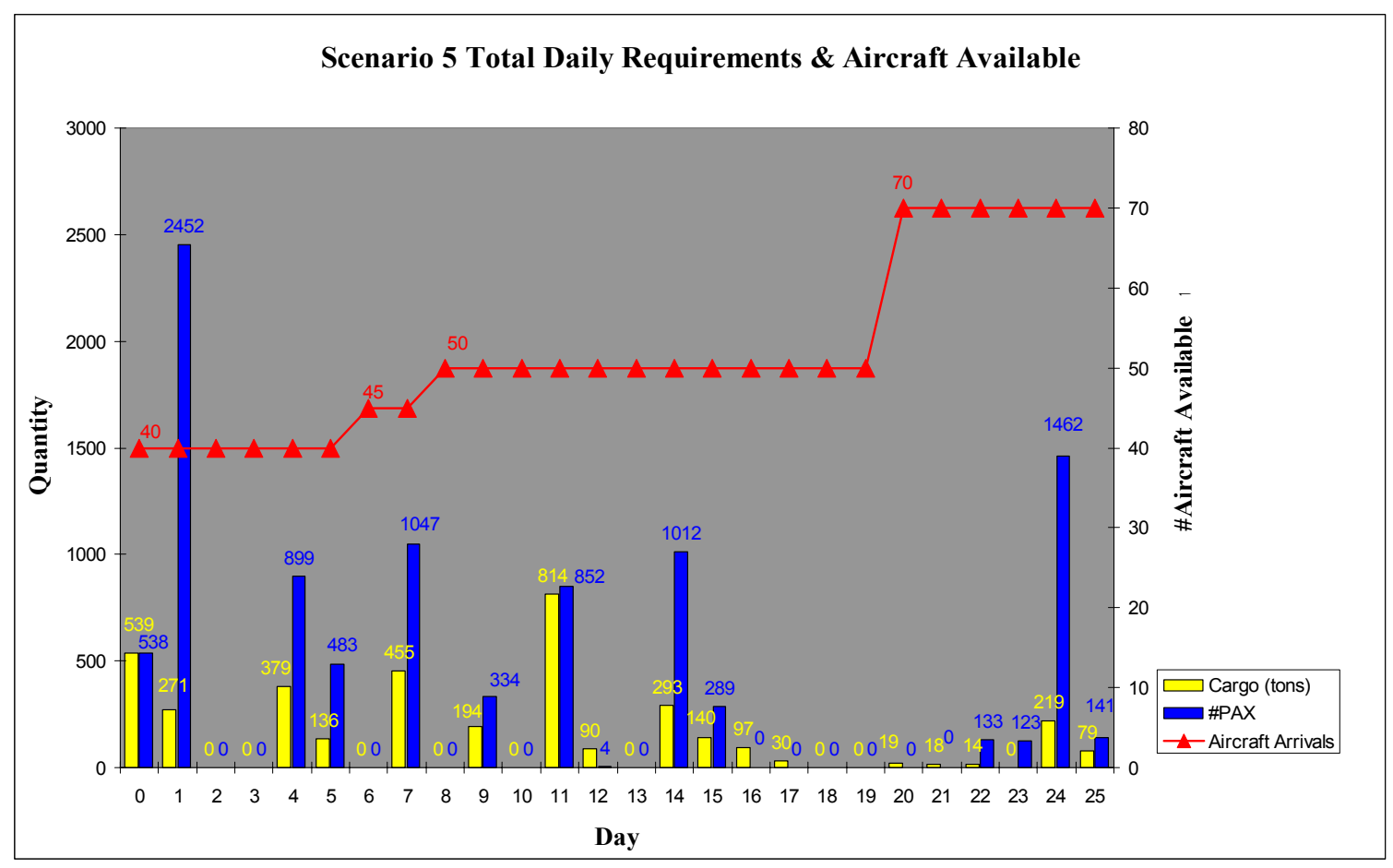

Figure 5.1 Example Problem Requirements and Aircraft Available 
The deployment horizon begins with the first requirement ALD at day zero and ends with the last RDD at day 32. Forty-nine requirements composed of 20 different commodities yield a total requirement of 3786 tons of cargo and 9769 PAX. The airlift network is summarized in Table 5.4.

\begin{tabular}{|c|c|}
\hline Type Arc & Number of Route Segments \\
\hline HB-APOE & 8 \\
\hline APOE-APOD & 40 \\
\hline APOD-REC & 5 \\
\hline REC-HB & 107 \\
\hline REC-APOE & 64 \\
\hline
\end{tabular}

Table 5.4 Example Problem Route Segments

Based upon the number of home bases, APOEs, APODs, recovery bases, aircraft types and route segments, a total of 1,228 arcs were generated to define the airlift network for Scenario 5.

\subsubsection{SAP-TS Results for Example Problem}

Tabu search settings for Scenario 5 are summarized in Table 5.5. These settings are the same as the default settings listed in Chapter 4 and are provided here for convenience.

Table 5.6 presents the initial solution objective function values. The initial solution is infeasible due to a working MOG violation of 451 and a UTE rate violation of 1 . The makespan for the initial solution is 27.95 days. 


\begin{tabular}{|c|c|c|}
\hline Parameter & Default Setting & Description \\
\hline NoChangeMovesTolerance & 3 & $\begin{array}{l}\text { \# no change value moves allowed } \\
\text { before diversifying }\end{array}$ \\
\hline UnimprovingMovesTolerance & 3 & $\begin{array}{l}\text { \# unimproving moves allowed before } \\
\text { diversifying }\end{array}$ \\
\hline SuperDiversifyTolerance & 10 & $\begin{array}{l}\text { \# small change moves allowed } \\
\text { before superdiversifying }\end{array}$ \\
\hline SolutionHistoryLength & 20 & $\begin{array}{l}\text { \# previous objectives to consider for } \\
\text { superdiverisfication }\end{array}$ \\
\hline ObjectiveTolerance & 0.01 & $\begin{array}{l}\text { tolerance for objective in } \\
\text { superdiversification count }\end{array}$ \\
\hline ChooseFirstImprovingMove & TRUE & $\begin{array}{l}\text { boolean for selecting the first } \\
\text { improving move }\end{array}$ \\
\hline MaxCompTime & 40 & $\begin{array}{l}\text { maximum search time (hours) alotted } \\
\text { for the search }\end{array}$ \\
\hline NumAircraftTrips & 12 & \# aircraft trips per aircraft \\
\hline NumRoutesToConsider & 50 & $\begin{array}{l}\text { maximum number of routes to create } \\
\text { for each arc }\end{array}$ \\
\hline MsnAssignmentPhase & TRUE & $\begin{array}{l}\text { boolean that sets mission assignment } \\
\text { phase status }\end{array}$ \\
\hline MsnImprovementPhase & FALSE & $\begin{array}{l}\text { boolean that sets mission } \\
\text { improvement phase status }\end{array}$ \\
\hline MsnAssignmentNBHSizeLimit & 100 & $\begin{array}{l}\text { mission Assignment Phase } \\
\text { maximum NBH size }\end{array}$ \\
\hline msnImprovementNBHSizeLimit & 800 & $\begin{array}{l}\text { mission Improvement Phase } \\
\text { maximum NBH size }\end{array}$ \\
\hline WorkingMOGTolerance & 0 & $\begin{array}{l}\text { threshold below which working } \\
\text { MOG feasible }\end{array}$ \\
\hline FuelMOGTolerance & 0 & $\begin{array}{l}\text { threshold below which fuel MOG } \\
\text { feasible }\end{array}$ \\
\hline UteRateTolerance & 0 & $\begin{array}{l}\text { threshold below which UTE rate } \\
\text { feasible }\end{array}$ \\
\hline NumberOfIterations & 800 & \# tabu search iterations \\
\hline Tenure & 7 & duration (iterations) of tabu attribute \\
\hline EliteListSize & 3 & number of elite solutions to retain \\
\hline ObjFnWeights & $\{1,1,1,1,1,1,1,1,1,1\}$ & $\begin{array}{l}\text { weights for changing priority of } \\
\text { objective values }\end{array}$ \\
\hline loadReallocationMoveDistance & 3 & $\begin{array}{l}\text { temporal distance (days) for LR } \\
\text { moves }\end{array}$ \\
\hline InsertDistance & 5 & $\begin{array}{l}\text { maximum insert distance (left \& } \\
\text { right) for WPI moves }\end{array}$ \\
\hline
\end{tabular}

Table 5.5 Example Problem Tabu Search Settings 


\begin{tabular}{|l|r|}
\hline \multicolumn{1}{|c|}{ Criterion } & Value \\
\hline Undelivered cargo penalty & 0.00 \\
\hline Late Delivery penalty & 9.72 \\
\hline workingMOGViolation & 451.00 \\
\hline Fuel MOG violation & 0.00 \\
\hline Total Number of missions & 159.00 \\
\hline Total Number of trivial loads & 17.00 \\
\hline Trivial load penalty & 64.21 \\
\hline Total Number of Aerial refueling missions & 0.00 \\
\hline UTE Rate violation & 1.00 \\
\hline Total Objective value & 701.93 \\
\hline
\end{tabular}

Table 5.6 Example Problem Initial Solution Objective

A total of 800 tabu search iterations were performed. The best solution mission summary is presented in Appendix A. The best solution objective function values, shown in Table 5.7, were obtained at iteration 384 in 3 hours and 33 minutes.

The best solution, with a makespan of 26.3 days, achieves a $74 \%$ reduction in the initial solution objective value and is feasible with respect to UTE rate and fuel MOG constraints. It is near feasible with a small working MOG violation of 16 . 


\begin{tabular}{|l|r|}
\hline \multicolumn{1}{|c|}{ Criterion } & Value \\
\hline Undelivered cargo penalty & 0.00 \\
\hline Late Delivery penalty & 16.24 \\
\hline workingMOGViolation & 16.00 \\
\hline Fuel MOG violation & 0.00 \\
\hline Total Number of missions & 138.00 \\
\hline Total Number of trivial loads & 3.00 \\
\hline Trivial load penalty & 9.50 \\
\hline Total Number of Aerial refueling missions & 0.00 \\
\hline UTE Rate violation & 0.00 \\
\hline Total Objective value & 182.74 \\
\hline
\end{tabular}

Table 5.7 Example Problem Best Solution Objective

\subsubsection{AMOS Results for Example Problem}

For comparison the AMOS results were evaluated using the SAP-TS objective function. AMOS and SAP-TS results are given in Table 5.8.

The SAP-TS best solution achieves an overall $83 \%$ reduction in the AMOS objective value greatly reducing late cargo (a critical issue for combatant commanders) and achieving better results for feasibility constraints. The AMOS makespan of 30.85 days is 4.55 days greater than the SAP-TS best solution. Figure 5.2 depicts the daily cumulative required total tonnage and the associated totals obtained with AMOS and SAP-TS. 


\begin{tabular}{|l|r|r|}
\hline \multicolumn{1}{|c|}{ Criterion } & AMOS Value & SAP-TS Value \\
\hline Undelivered cargo penalty & 0.00 & 0.00 \\
\hline Late Delivery penalty & 762.97 & 16.24 \\
\hline workingMOGViolation & 58.00 & 16.00 \\
\hline Fuel MOG violation & 0.00 & 0.00 \\
\hline Total Number of missions & 116.00 & 138.00 \\
\hline Total Number of trivial loads & 19.00 & 3.00 \\
\hline Trivial load penalty & 136.92 & 9.50 \\
\hline Total Number of Aerial refueling missions & 0.00 & 0.00 \\
\hline UTE Rate violation & 0.00 & 0.00 \\
\hline Total Objective value & 1092.89 & 182.74 \\
\hline
\end{tabular}

Table 5.8 Example Problem AMOS \& SAP-TS Objective 


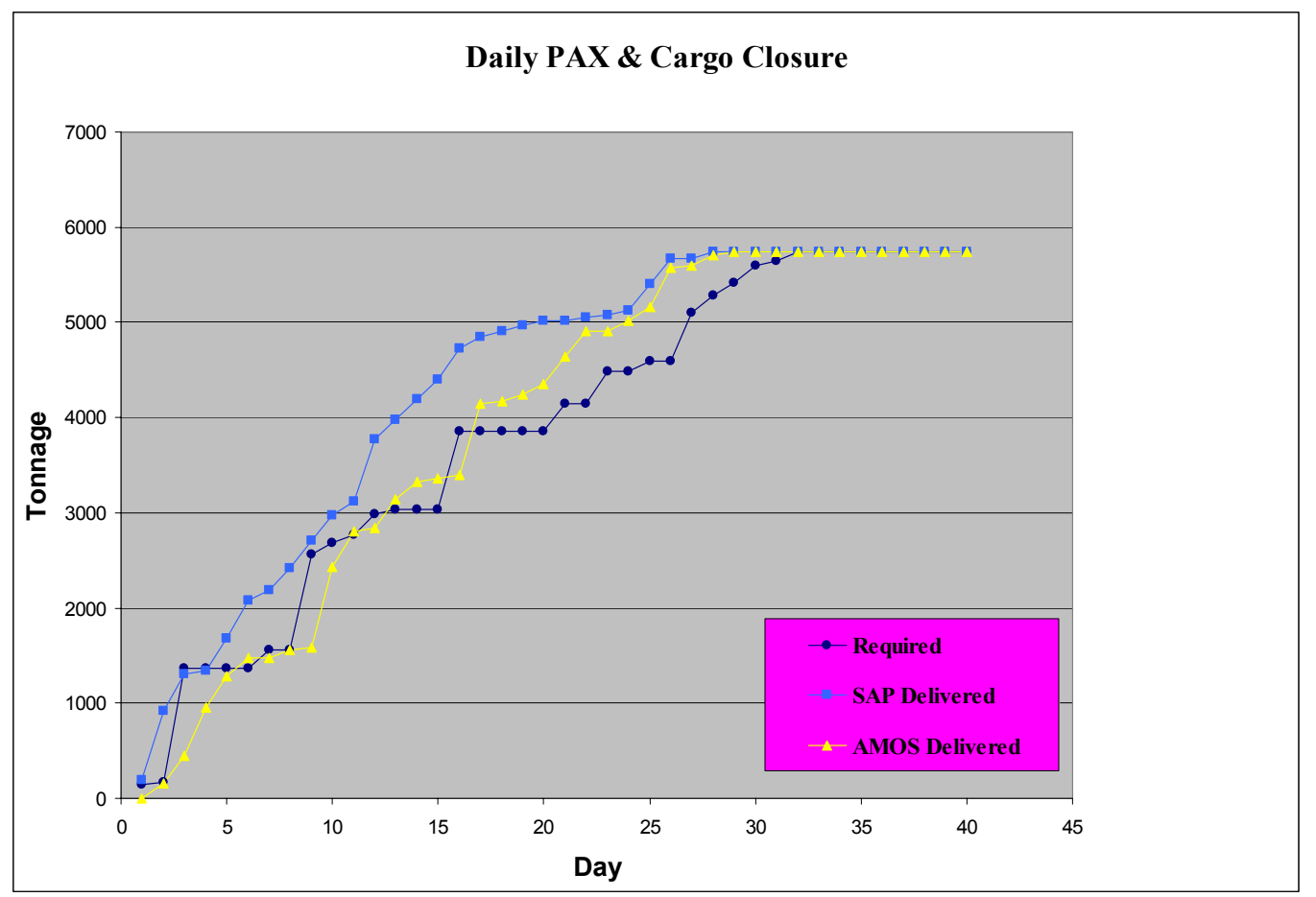

Figure 5.2 Example Problem PAX \& Cargo Closure

SAP-TS dominates AMOS throughout the time span of the deployment and incurs a single instance of delivery time shortfall.

\subsection{OTHER SAP PROBLEM INSTANCES}

Scenarios 1 through 4 were run using the SAP-TS settings indicated in Table 5.5 with the following exceptions. Each scenario was run for a total of 15 hours. The standard tabu search phase was capped at 12 hours. After the standard tabu search phase, a series of elite iterations were performed starting with the best solution found during the 12 hour phase. Elite iteration neighborhoods were restricted to the mission improvement phase neighborhoods-LR, WMAS, ITD, and RAI to intensify the search around the 
elite solution. The number of elite iterations performed is the smaller of 800 iterations and the number of iterations performed in 3 hours.

AMOS and SAP-TS results for the Scenarios 1 through 4 are summarized in Table 5.9.

\begin{tabular}{|c|c|c|c|c|c|c|c|c|}
\hline \multirow[t]{2}{*}{ Criterion } & \multicolumn{2}{|c|}{ Scenario 1} & \multicolumn{2}{|c|}{ Scenario 2} & \multicolumn{2}{|c|}{ Scenario 3} & \multicolumn{2}{|c|}{ Scenario 4} \\
\hline & AMOS & TS & AMOS & TS & AMOS & TS & AMOS & TS \\
\hline $\begin{array}{r}\text { Undelivered } \\
\text { cargo }\end{array}$ & 144.40 & 0.00 & 0.80 & 0.00 & 4.95 & 0.00 & 0.00 & 0.00 \\
\hline Late Cargo & 7005.83 & 69.61 & 9992.05 & 8978.00 & 20974.74 & 749.55 & 1595.08 & 135.20 \\
\hline $\begin{array}{r}\text { Working } \\
\text { MOG }\end{array}$ & 163.00 & 114.00 & 1038.00 & 1033.00 & 578.00 & 314.00 & 51.00 & 41.00 \\
\hline Fuel MOG & 0.00 & 0.00 & 0.00 & 0.00 & 0.00 & 0.00 & 0.00 & 0.00 \\
\hline \# Missions & 240.00 & 248.00 & 425.00 & 389.00 & 577.00 & 403.00 & 116.00 & 228.00 \\
\hline $\begin{array}{r}\text { \# Trivial } \\
\text { Loads }\end{array}$ & 56.00 & 12.00 & 88.00 & 34.00 & 239.00 & 22.00 & 19.00 & 2.00 \\
\hline $\begin{array}{r}\text { Trivial Load } \\
\text { penalty }\end{array}$ & 507.89 & 27.00 & 805.28 & 348.32 & 1837.79 & 71.70 & 136.92 & 1.51 \\
\hline $\begin{array}{r}\# \text { Aerial } \\
\text { Refueling } \\
\text { missions }\end{array}$ & 0.00 & 0.00 & 0.00 & 0.00 & 0.00 & 0.00 & 0.00 & 0.00 \\
\hline $\begin{array}{r}\text { UTE Rate } \\
\text { violation }\end{array}$ & 4237.00 & 141.00 & 30096.00 & 5069.00 & 12531.00 & 2718.00 & 1623.00 & 11.00 \\
\hline $\begin{array}{r}\text { Total } \\
\text { Objective } \\
\text { value }\end{array}$ & 12354.12 & 611.61 & 42445.13 & 15851.32 & 36742.48 & 4278.25 & 3541.00 & 418.71 \\
\hline Makespan & 31.52 & 26.99 & 18.40 & 18.82 & 40.47 & 32.35 & 29.02 & 28.62 \\
\hline
\end{tabular}

Table 5.9 AMOS and SAP-TS Results for Scenarios 1 through 4

\subsubsection{Scenario 1}

Scenario 1 consists of 59 requirements totaling 7,647 tons of cargo and 14,769 PAX that become available for delivery over 25 days. A total of 166 aircraft are available at the start building to a final total of 297 aircraft available at day 20. Requirement and aircraft arrival details are shown in Figure 5.3. 


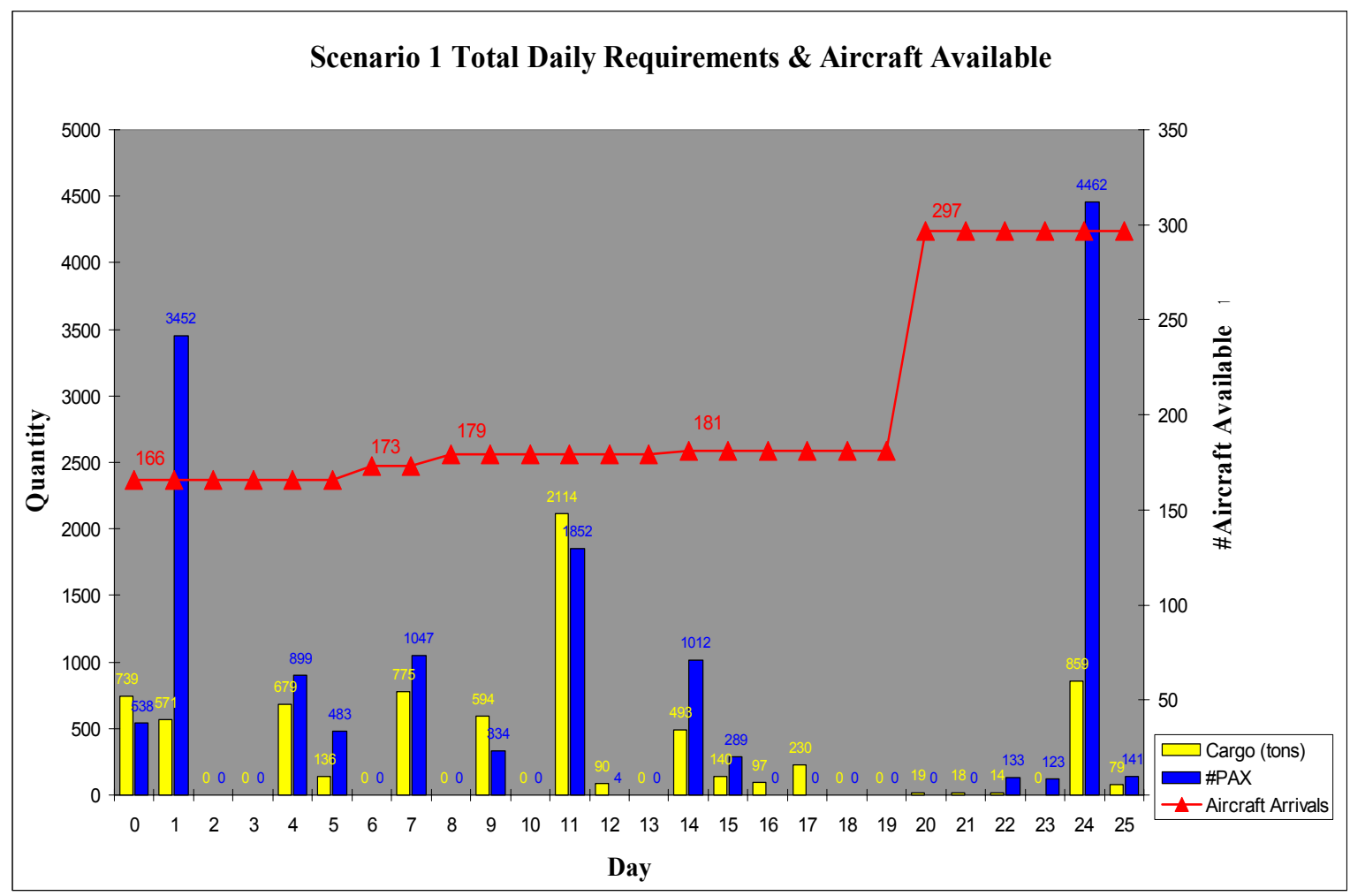

Figure 5.3 Scenario 1 Total Daily Requirements and Aircraft Available

The Scenario 1 best solution was found at iteration 509 after 12 hours and 43 minutes. The Scenario 1 SAP-TS best solution achieves an overall 95\% reduction in the AMOS objective value, a reduction of $99 \%$ in late cargo penalty, and no undelivered cargo. SAP-TS delivers (closes) all requirements 4.53 days earlier than AMOS. Figure 5.4 provides a comparison of the closure rates for AMOS and SAP-TS for Scenario 1. 


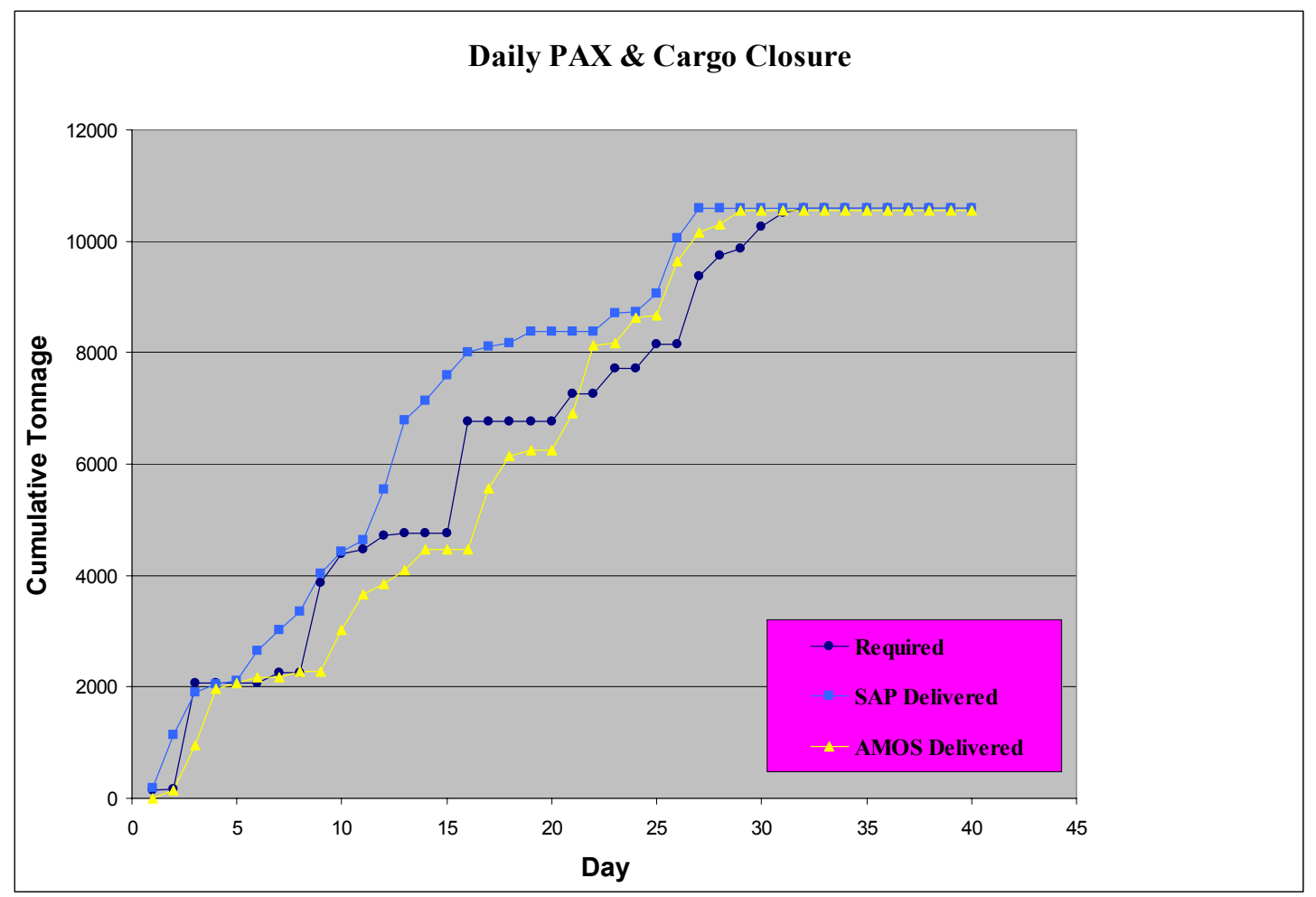

Figure 5.4 Scenario 1 PAX \& Cargo Closure

\subsubsection{Scenario 2}

Scenario 2 consists of 132 requirements totaling 15,482 tons of cargo and 10,576 PAX that become available for delivery over 10 days. Aircraft available start at 60 aircraft on day 0 and build to a final total of 124 aircraft by day 8 . Scenario 2 is the most challenging scenario in that requirements become available in less than half the time (10 days vice 25$)$ of the other scenarios and the available aircraft are not commensurate with the total requirement. Requirement and aircraft arrival details for Scenario 2 are shown in Figure 5.5. 


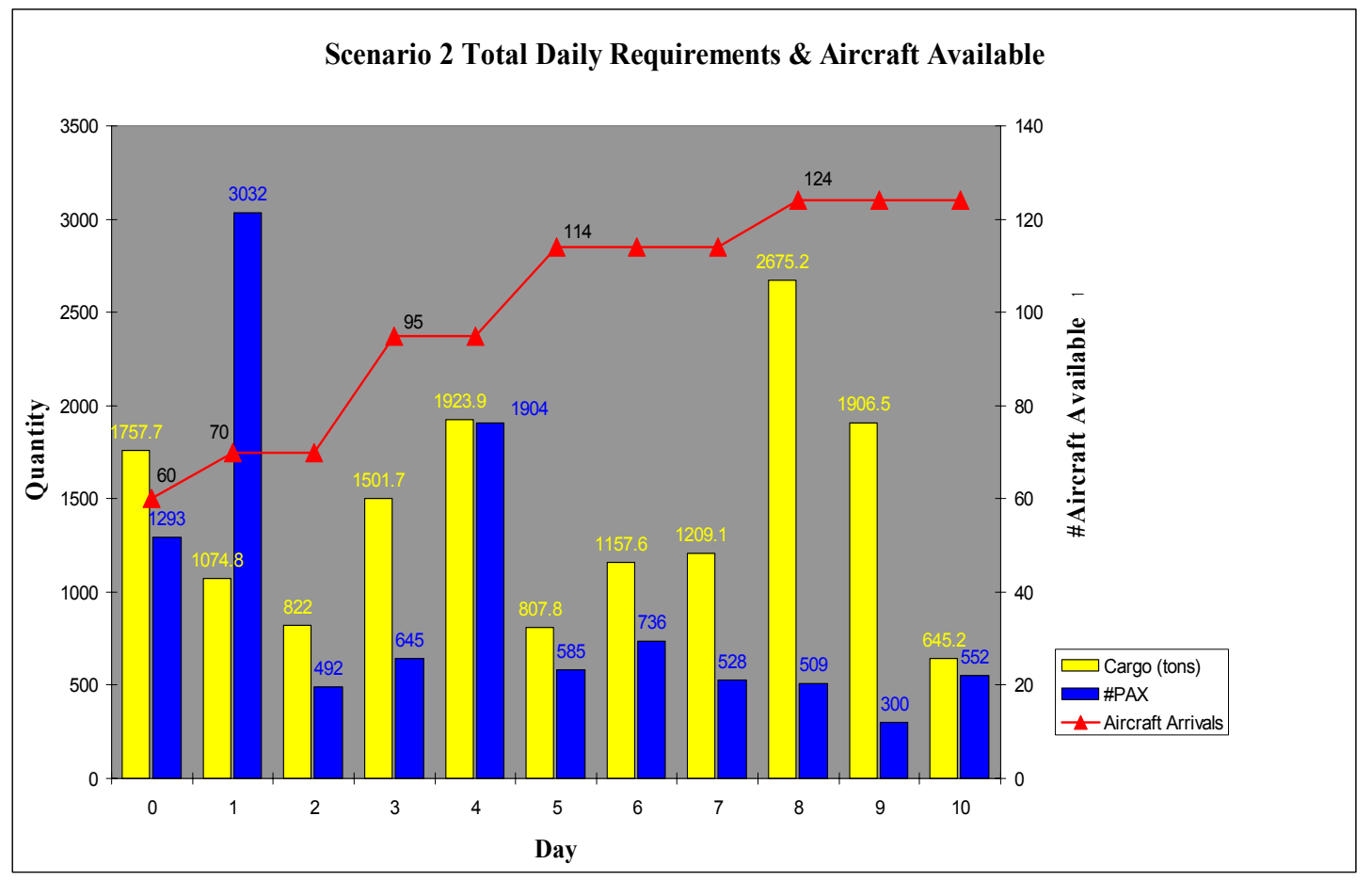

Figure 5.5 Scenario 2 Total Daily Requirements and Aircraft Available

The Scenario 2 best solution was found at iteration 134 after 9 hours and 31 minutes. The Scenario 2 SAP-TS best solution achieves an overall $63 \%$ reduction in the AMOS objective value and a reduction of $10 \%$ in late cargo penalty. The SAP-TS solution closed 10 hours later than the AMOS solution. Figure 5.6 provides a comparison of the closure rates for AMOS and SAP-TS for Scenario 2. Further analysis of Scenario 2 is provided in Section 5.4. 


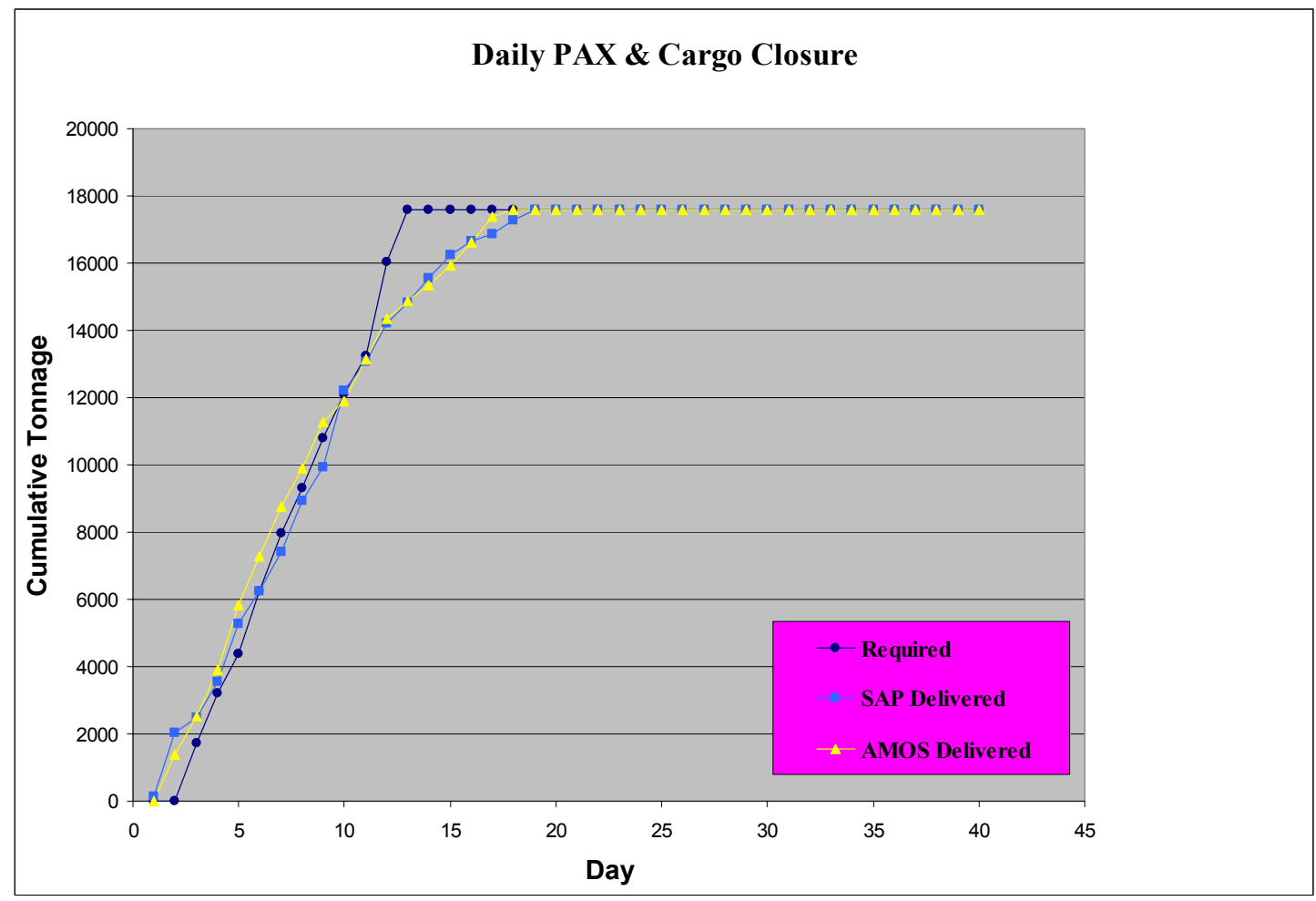

Figure 5.6 Scenario 2 PAX \& Cargo Closure

\subsubsection{Scenario 3}

Scenario 3 consists of 96 requirements totaling 13,777 tons of cargo and 16,683 PAX that become available for delivery over 25 days. Aircraft available start at 166 aircraft on day 0 and build to a final total of 297 aircraft by day 20 . Requirement and aircraft arrival details for Scenario 3 are shown in Figure 5.7. 


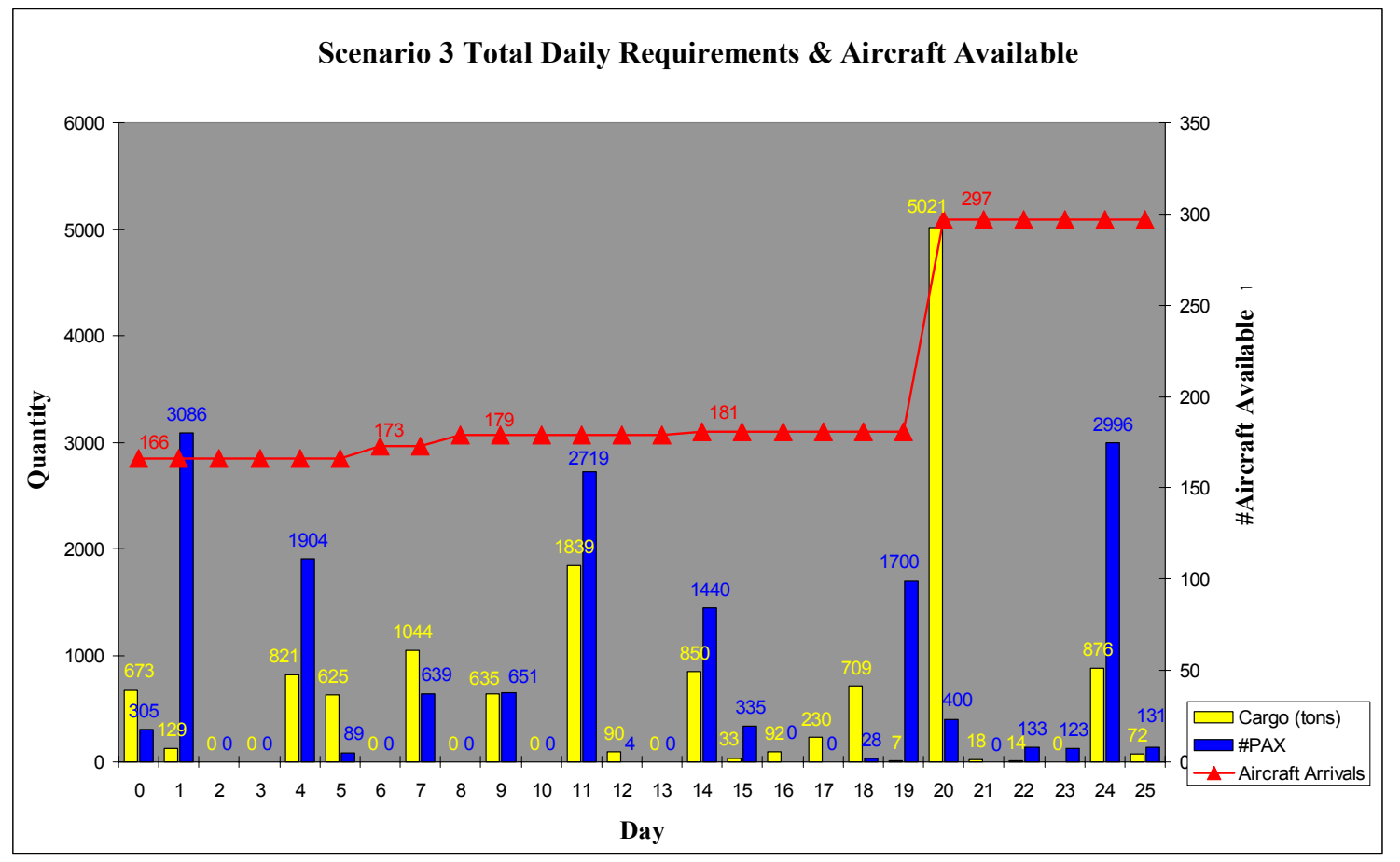

Figure 5.7 Scenario 3 Total Daily Requirements and Aircraft Available

The Scenario 3 best solution was found at iteration 908 after 15 hours. The Scenario 3 SAP-TS best solution achieves an overall $88 \%$ reduction in the AMOS objective value and a reduction of $96 \%$ in late cargo penalty with no undelivered cargo. The SAP-TS solution closed 9.6 hours earlier than the AMOS solution. Figure 5.8 provides a comparison of the closure rates for AMOS and SAP-TS for Scenario 3. 


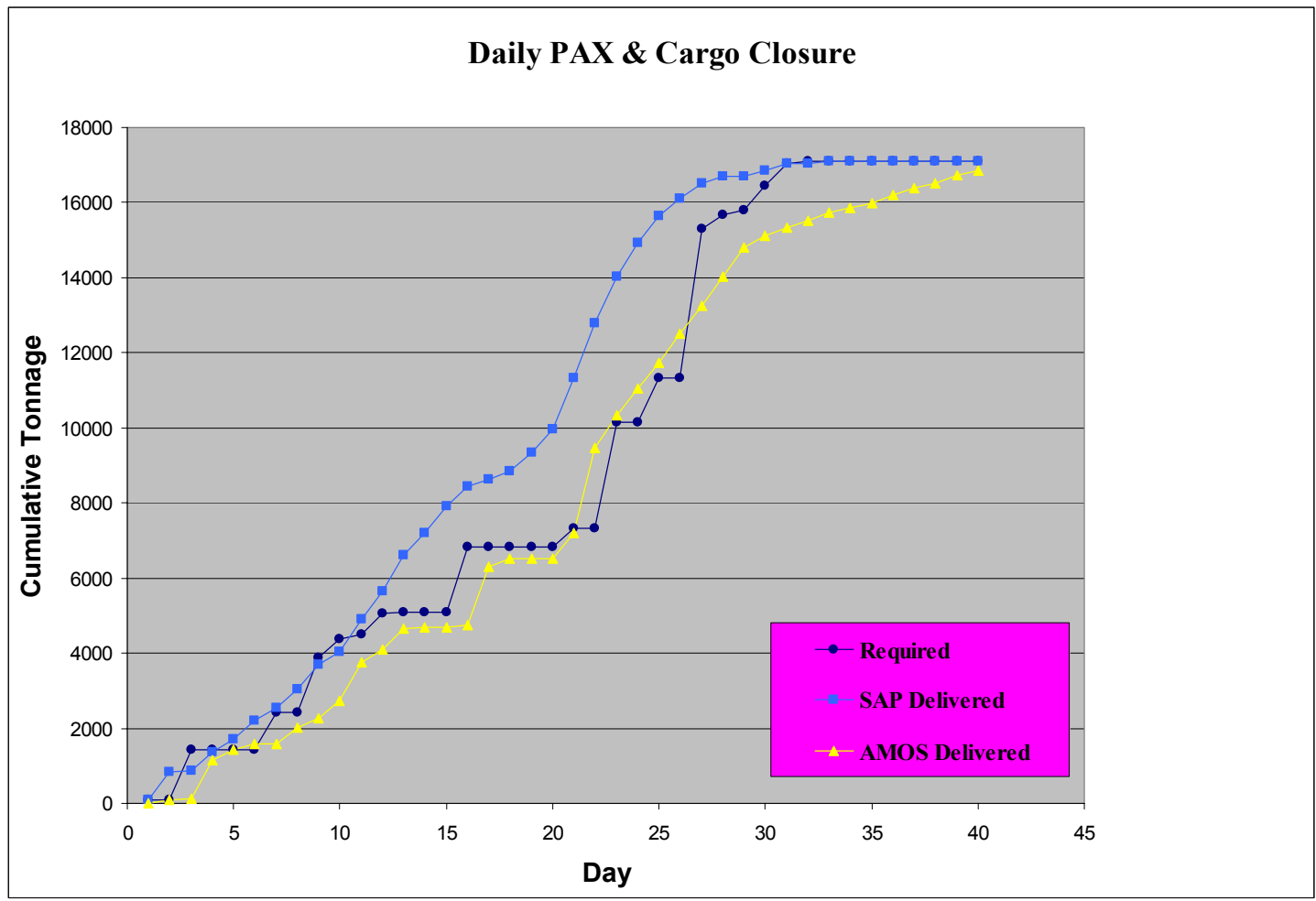

Figure 5.8 Scenario 3 PAX \& Cargo Closure

\subsubsection{Scenario 4}

Scenario 4 consists of 59 requirements totaling 7,647 tons of cargo and 14,769 PAX that become available for delivery over 25 days. Aircraft available start at 91 aircraft on day 0 and build to a final total of 165 aircraft by day 20 . Requirement and aircraft arrival details for Scenario 4 are shown in Figure 5.9. 


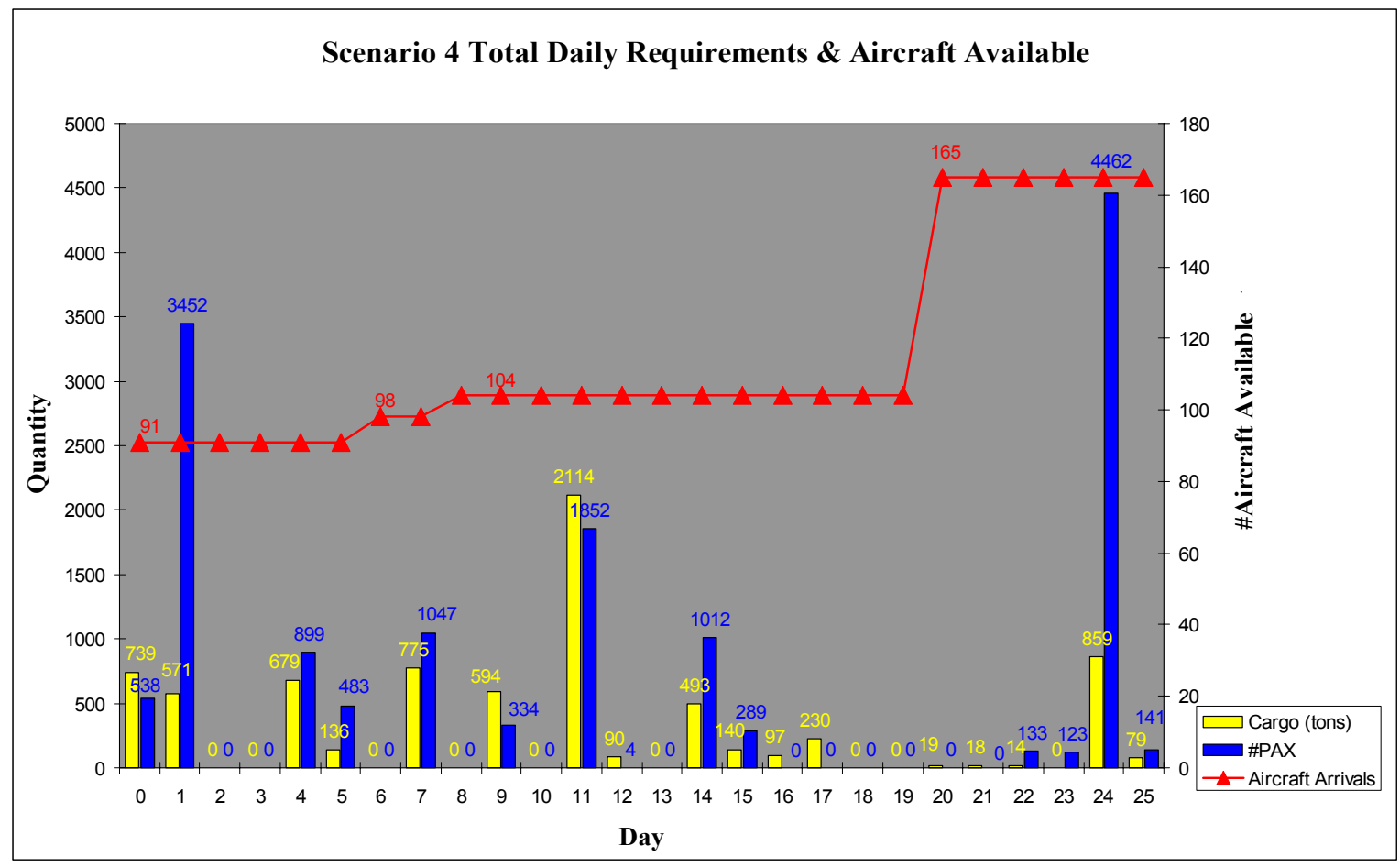

Figure 5.9 Scenario 4 Total Daily Requirements and Aircraft Available

The Scenario 4 best solution was found at iteration 270 after 4 hours and 38 minutes. The Scenario 4 SAP-TS best solution achieves an overall $88 \%$ reduction in the AMOS objective value and a reduction of $92 \%$ in late cargo penalty with no undelivered cargo. The SAP-TS solution closed 8.4 days earlier than the AMOS solution. Figure 5.10 provides a comparison of the closure rates for AMOS and SAP-TS for Scenario 4. 


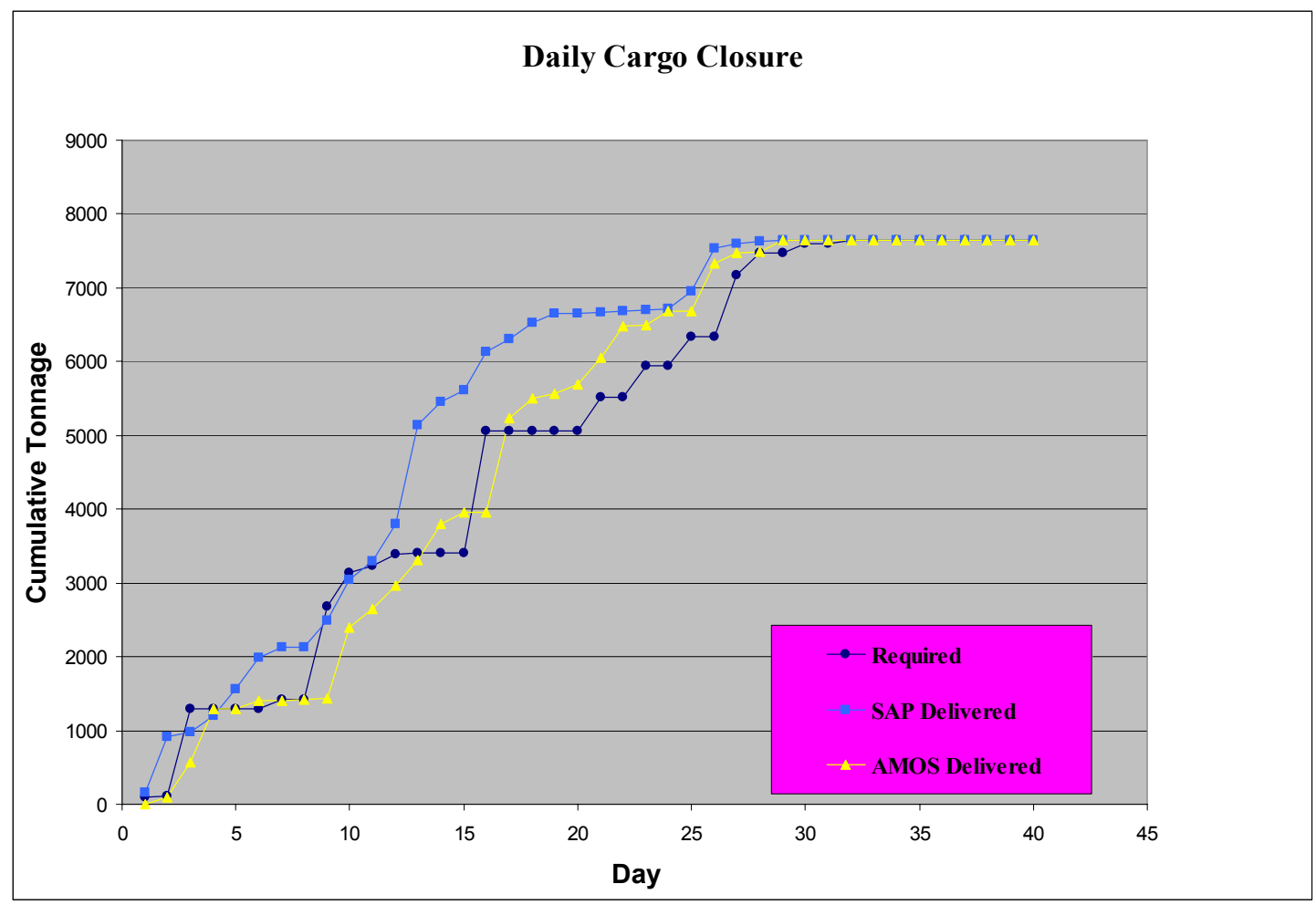

Figure 5.10 Scenario 4 PAX \& Cargo Closure

\subsection{EXTENDED ANALYSIS OF SCENARIO 2}

Scenario 2 represented the most challenging deployment amongst the 5 different scenarios. The requirement time windows spanned a minimum ALD of day 0 to a max RDD of 10 - a 10 day horizon for the deployment. Along with this compressed horizon, the number of aircraft allocated is insufficient to meet the requirement demand within the given time windows. As a result, both AMOS and SAP-TS solutions incurred a substantial late delivery penalty. A snapshot of the SAP-TS solution deliveries juxtaposed with requirement time windows is 
shown in Figure 5.11. Significant delays in requirement delivery begin to occur at about day 8 however the origin of these delays starts at around day 4 .

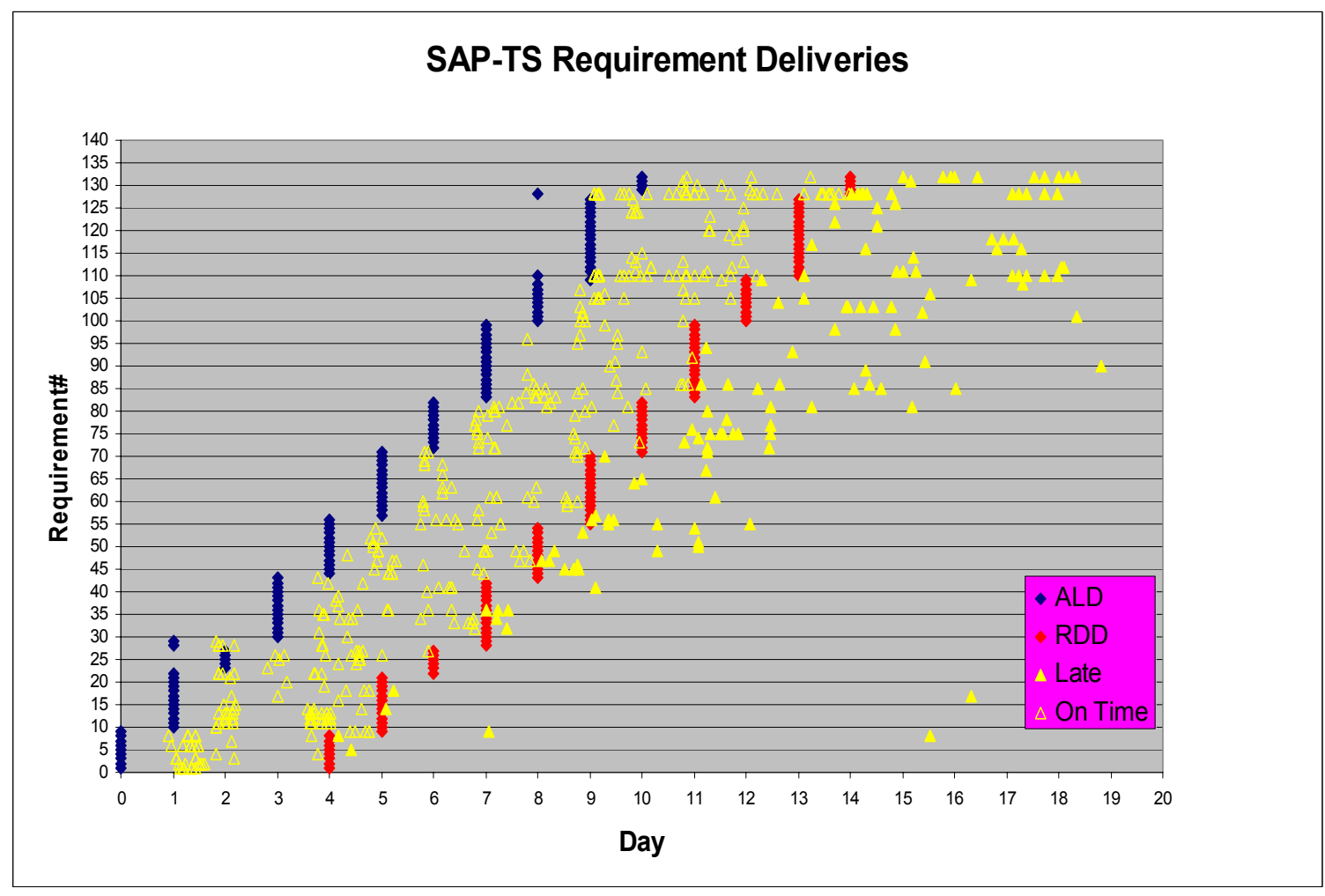

Figure 5.11 SAP-TS Scenario 2 Solution with Time windows

The following is an extended analysis of the SAP-TS best solution for Scenario2. This analysis is performed to illustrate how SAP-TS results can be used to gain additional insight into a particular SAP instance. What additional resources are needed to better meet deployment demands? Which bases are restricting the flow of aircraft through the network? These are some of the questions analysts are interested in when developing a strategic airlift plan. For the purposes of this discussion, we confine our study to determining what changes 
in available aircraft and working MOG at critical bases will improve closure times of the requirements.

The number of aircraft required to move all requirements within their respective time windows is a function of the requirements themselves (commodity type; amount of outsize, oversize, bulk and PAX), aircraft capabilities (ACL, cargo compatibility, UTE rates), and the strategic airlift network (critical legs, MOG, number of routes). We use the $\mathrm{C} 17$ as the base aircraft to determine approximately how many $\mathrm{C} 17$ missions are required to deliver all requirements. The planning payloads for the $\mathrm{C} 17$ are described in Table 3 of Air Mobility Planning Factors (Air Force Pamphlet 10-1403, 1998). These are: 45 tons of cargo with 90 passengers and 102 passengers if there is no cargo on board. These payloads represent historical averages from Desert Shield/Desert Storm. Since all Scenario 2 APOE-APOD pairs are from CONUS to Southwest Asia, these payloads will be adequate for obtaining a rough estimate of the number of $\mathrm{C} 17$ missions required.

The daily cargo and PAX requirements for Scenario 2 are shown in Figure 5.5. Using the $\mathrm{C} 17$ planning factors outlined above, the calculation for the number of $\mathrm{C} 17$ missions required to deliver all cargo and PAX is defined by the following function:

$$
f(\text { cargo, } p a x)=\lceil\operatorname{cargo} / 45\rceil+ \begin{cases}\lceil(\operatorname{pax}-\lceil\operatorname{cargo} / 45\rceil 90) / 102 & p a x-\lceil\operatorname{cargo} / 45\rceil 90>0 \\ 0, & p a x-\lceil\operatorname{cargo} / 45\rceil 90 \leq 0\end{cases}
$$

where cargo is the amount of cargo in tons and pax is the number of personnel. 
As an example, we calculate the number of $\mathrm{C} 17$ missions required to move the total requirements available on day 0 (ignoring the APOE-APOD pairs): 1,757.7 tons of cargo and 1,293 PAX. First calculate the number of cargo missions: $1757.7 / 45=39.06$ cargo missions or 40 missions total. These cargo missions may carry $40 * 90$ or 3600 total PAX. Since this exceeds the 1,293 PAX on hand, the PAX are delivered using the cargo missions and no additional PAX only aircraft are necessary. C17 mission requirements for the remaining days (again, ignoring APOE-APOD pairs) are shown in Table 5.10.

\begin{tabular}{|c|c|c|c|}
\hline DAY & CARGO (tons) & \#PAX & \# C17 Missions \\
\hline 0 & 1757.7 & 1293 & 40 \\
\hline 1 & 1074.8 & 3032 & 33 \\
\hline 2 & 822.0 & 492 & 19 \\
\hline 3 & 1501.7 & 645 & 34 \\
\hline 4 & 1923.9 & 1904 & 43 \\
\hline 5 & 807.8 & 585 & 18 \\
\hline 6 & 1157.6 & 736 & 26 \\
\hline 7 & 1209.1 & 528 & 27 \\
\hline 8 & 2675.2 & 509 & 60 \\
\hline 9 & 1906.5 & 300 & 43 \\
\hline 10 & 645.2 & 552 & 15 \\
\hline TOTALS & $\mathbf{1 5 4 8 2}$ & $\mathbf{1 0 5 7 6}$ & $\mathbf{3 5 8}$ \\
\hline
\end{tabular}

Table 5.10 Number of Required C17 Missions by Day for Scenario 2 


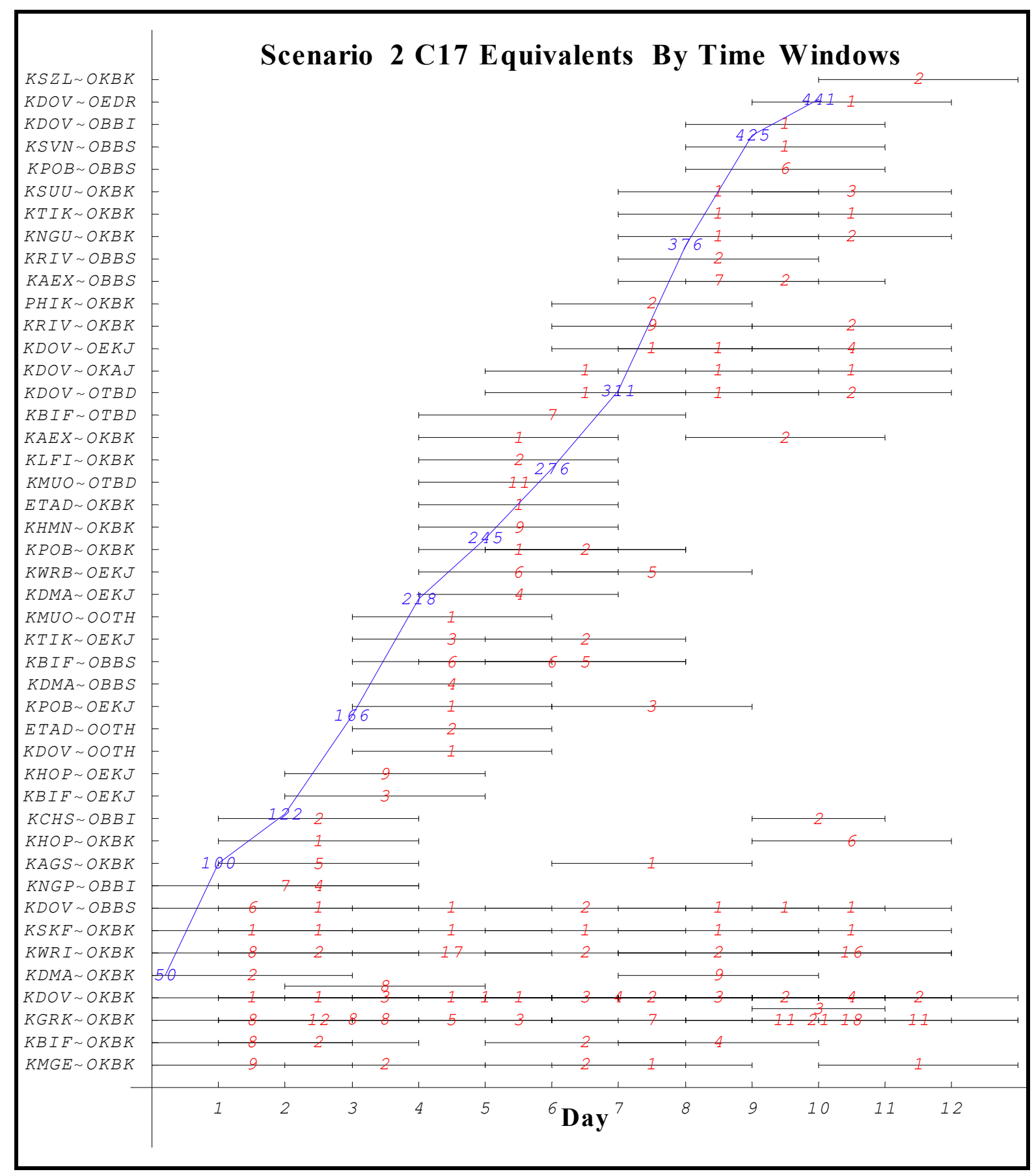

Figure 5.12 C17 Missions by Time Window and APOE-APOD Pair 
The total number of $\mathrm{C} 17$ missions required is 358 . Determining the number of required $\mathrm{C} 17$ missions based solely upon daily cargo available is admittedly a gross simplification. The results in Table 5.10 do not take into account the impact of different APOE-APOD pairs (nor loading issues due to commodity type and base constraints which we ignore here). Figure 5.12 depicts the impact of multiple APOE-APOD pairs on the number of missions required. Requirements with the same time window and APOE-APOD pair are grouped together. The red number on the time window represents the number of $\mathrm{C} 17$ missions needed to deliver the requirements for the given time window and APOE-APOD pair. The blue line (and numbers) indicates the cumulative number of $\mathrm{C} 17$ missions required over time.

The total number of $\mathrm{C} 17$ missions required has risen from 358 to 441 when the different APOE-APOD pairs are included. Scenario 2 aircraft available by type are shown in Table 5.11. The actual number of missions performed by SAP-TS and AMOS by type are shown in Figure 5.13 and Figure 5.14 respectively. 


\begin{tabular}{|c|c|c|}
\hline Day & Quantity & Type \\
\hline 0 & 5 & WBC \\
\hline 0 & 10 & WBP \\
\hline 0 & 10 & C-5A \\
\hline 0 & 10 & C-17 \\
\hline 0 & 10 & C-5A \\
\hline 0 & 15 & WBP \\
\hline 1 & 10 & WBC \\
\hline 3 & 10 & C-17 \\
\hline 3 & 15 & WBC \\
\hline 5 & 9 & C-5A \\
\hline 5 & 10 & C-17 \\
\hline 8 & 10 & C-5A \\
\hline
\end{tabular}

Table 5.11 Scenario 2 Aircraft Arrivals by Type

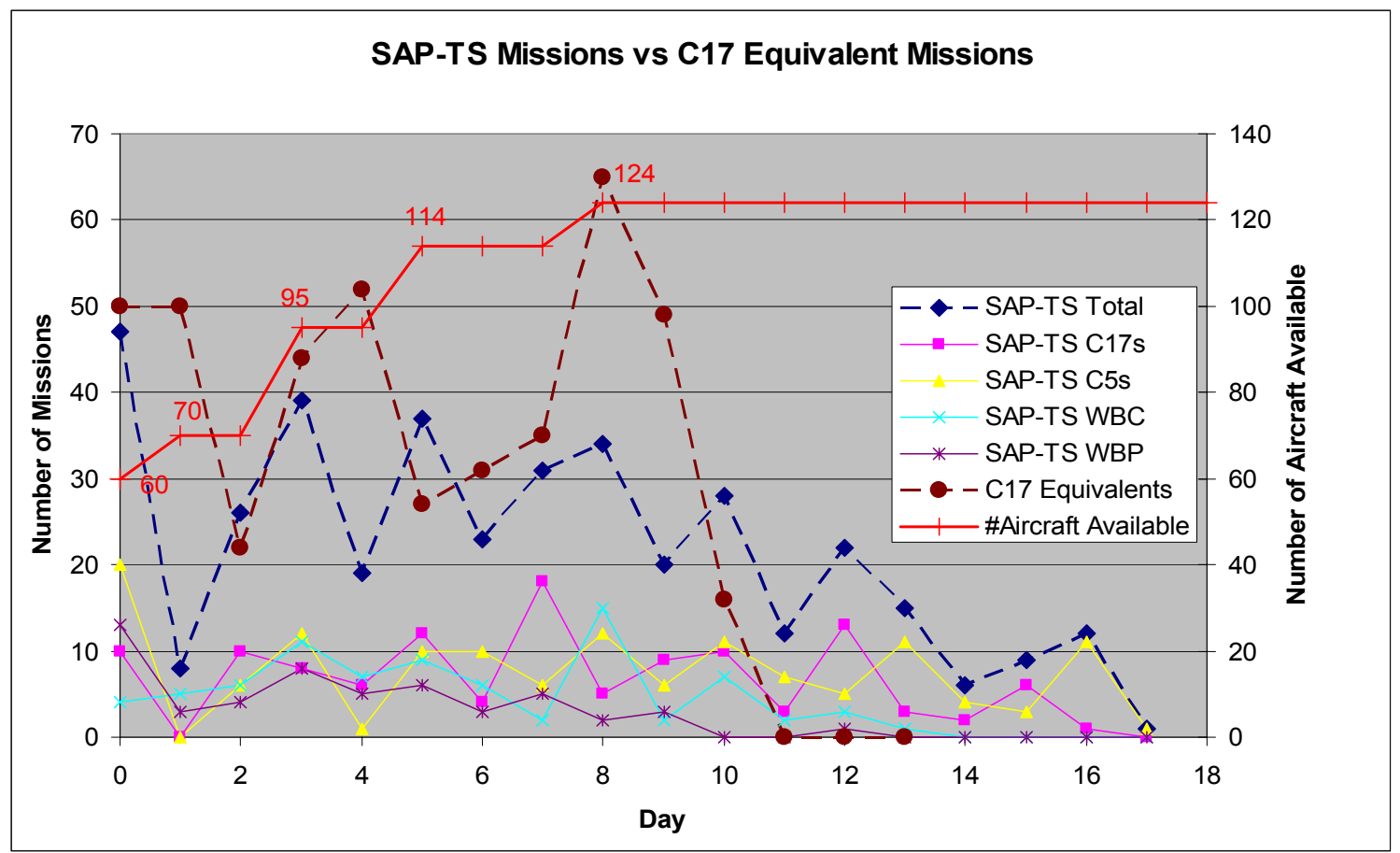

Figure 5.13 SAP-TS Missions vs C17 Equivalent Missions 


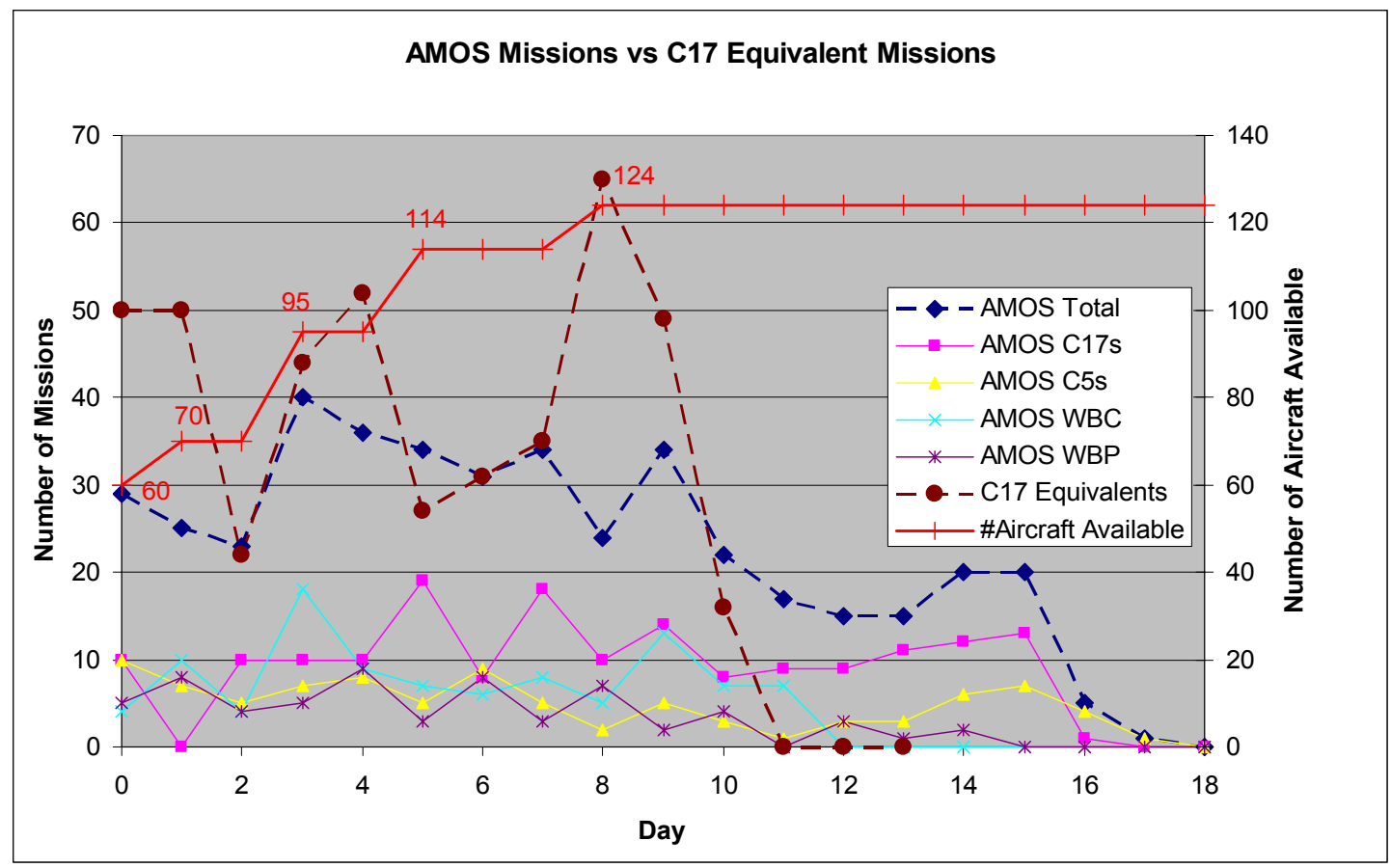

Figure 5.14 AMOS Missions vs C17 Equivalent Missions

A clear shortfall or "mission gap," measured as the difference between the C17 mission equivalents and the total missions performed in the respective solutions, is apparent in both solutions. The "mission gap" is most prominent between days 8 and 10 achieving a maximum shortfall of about $30 \mathrm{C} 17$ missions on day 8 for SAP-TS and $40 \mathrm{C} 17$ missions for AMOS on day 8 . Since the requirements during the period from day 8 to day 10 are cargo intensive (5227 tons of cargo, 1361 PAX, see Figure 5.5), an increase in the available number of $\mathrm{C} 5 \mathrm{~s}$ and/or $\mathrm{C} 17 \mathrm{~s}$ prior to or during this window would improve closure times of the requirements. 
A determination of the quantity and mix of additional aircraft must include the impact of airlift network constraints on aircraft throughput. Specifically, we investigate those bases with working MOG violations in the SAP-TS solution. These represent the bottlenecks or locations where aircraft flow is constrained due to working MOG limitations.

For the SAP-TS solution, 10 bases have working MOG violations totaling 1033. These bases along with the respective total working MOG violation and number of aircraft visits are summarized in Table 5.12.

\begin{tabular}{|c|c|c|c|c|}
\hline $\begin{array}{l}\text { Base } \\
\text { Name } \\
\end{array}$ & $\begin{array}{c}\text { Working MOG } \\
\text { (Wide/Logic/Narrow) }\end{array}$ & $\begin{array}{c}\text { MOG } \\
\text { Violation } \\
\end{array}$ & \#Visits & Base Role(s) \\
\hline ETAD & 1/OR/1 & 46 & 42 & APOE/ENROUTE \\
\hline OKBK & $5 / \mathrm{OR} / 5$ & 283 & 254 & APOD \\
\hline OBBS & $2 / \mathrm{OR} / 2$ & 42 & 52 & APOD \\
\hline OBBI & $2 / \mathrm{OR} / 2$ & 14 & 15 & APOD \\
\hline OEKJ & $2 / \mathrm{OR} / 2$ & 49 & 40 & APOD \\
\hline OTBD & $2 / \mathrm{OR} / 2$ & 14 & 20 & APOD \\
\hline LEMO & $3 / \mathrm{OR} / 4$ & 492 & 136 & REC/ENROUTE \\
\hline LICZ & $2 / \mathrm{OR} / 4$ & 7 & 57 & REC/ENROUTE \\
\hline EGVA & $1 / \mathrm{OR} / 1$ & 30 & 40 & ENROUTE \\
\hline HECA & $1 / \mathrm{OR} / 2$ & 56 & 34 & ENROUTE \\
\hline
\end{tabular}

Table 5.12 Scenario 2 Working MOG Violation Bases (SAP-TS) 
Half of the MOG violation bases for this solution are APOD bases, contributing about $39 \%$ of the total working MOG violation. APOD bases typically do not have large wide or narrow working MOG. Located in the theater of operations, APOD facilities are often limited when compared to CONUS bases. Of course a large number of APOD visits for requirement delivery are expected and thus these bases are likely to be the sources of bottlenecks in the airlift system. If the requirement time windows are tight as in this scenario, aircraft begin to compete for working MOG space.

LEMO accounts for $48 \%$ of the MOG violation - the largest single contributor among the bases. The 126 aircraft visits to LEMO are bested only by the 256 visits to OKBK. The high aircraft visit frequency for LEMO is because this base serves as the sole recovery base for $\mathrm{C}-5 \mathrm{~s}$ in the theater as well as an en route base for 23 of the 49 arcs servicing CONUS to theater and back again.

LICZ is one of the two recovery bases for $\mathrm{C} 17 \mathrm{~s}$ - the other is ETAR. LICZ has a small working MOG violation of 7 and ETAR has no working MOG violation in the SAP-TS solution for Scenario 2. 0 depicts the total number of aircraft that visit OKBK, LEMO and LICZ over time. Major spikes in the number of aircraft occur during days 1 to 3 and days 9 to 11 . Referring to Figure 5.13, we see that these time periods coincide with the "mission gaps." Increasing the number of $\mathrm{C} 5 \mathrm{~s}$ to bridge the mission gap during these periods would only increase working MOG violations at OKBK and LEMO. An increase in available C17s during these intervals would be better from a working MOG standpoint since LICZ and 
ETAR (recovery bases for $\mathrm{C} 17 \mathrm{~s}$ ) are less constrained and the $\mathrm{C} 17 \mathrm{~s}$ do not recover at LEMO.

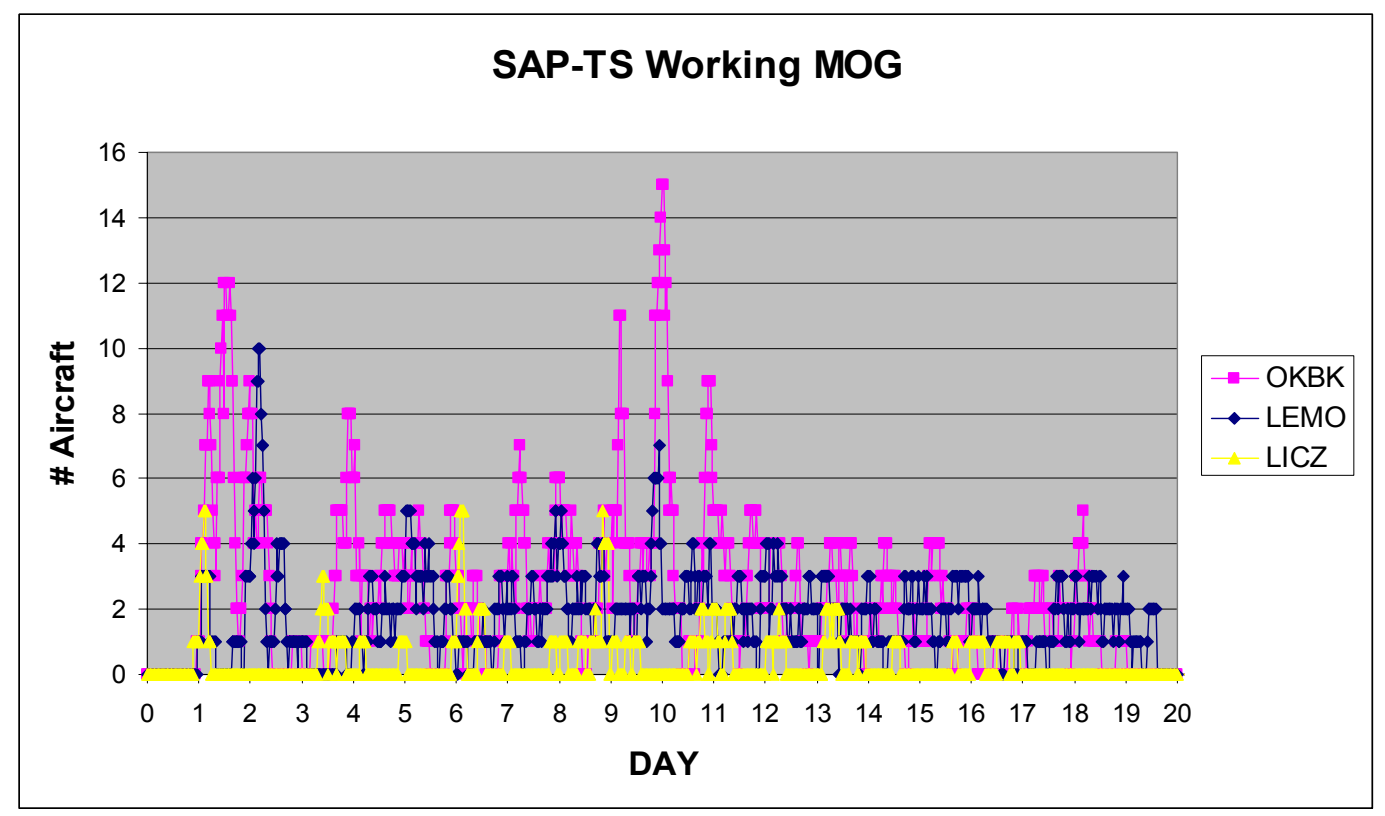

Figure 5.15 SAP-TS Working MOG for OKBK, LEMO and LICZ

Based upon this simple analysis of the SAP-TS solution, several broad conclusions can be made about the current strategic airlift plan for Scenario 2. First, additional aircraft are required to successfully meet requirement RDDs. Because the late requirements are predominantly cargo, the type of aircraft should be $\mathrm{C} 5 \mathrm{~s}$ or $\mathrm{C} 17 \mathrm{~s}$. Ideally, the additional aircraft should be made available no later than day 4 the time when late cargo begins to manifest itself. $\mathrm{C} 17 \mathrm{~s}$ are a better choice than $\mathrm{C} 5 \mathrm{~s}$ based upon the characteristics of the airlift system. Current C5 missions are straining base capacity at several key bases (LEMO, OKBK). Additional C5 missions will increase congestion in the network. Secondly, 
changes to the strategic airlift network to improve throughput are necessary to meet requirement delivery windows for this deployment. A quick fix would be to identify an alternate recovery base for C5s. ETAR, one of the C17 recovery bases, has a working MOG that can support 20 wide aircraft (C5s) and 20 narrow body aircraft $(\mathrm{C} 17 \mathrm{~s}$,$) simultaneously. If deemed suitable, ETAR would be an$ ideal alternate recovery base for C5s and would also help divert some missions away from LEMO. A longer term fix would be to identify bases in the airlift network that are likely to be bottlenecks, such as LEMO and the APOD bases and to make long term investments in facility improvements to increase the MOG capacity.

\subsection{CONCLUSION}

This chapter discussed the application of SAP-TS to 5 separate scenarios. SAP-TS results dominate AMOS results in virtually all aspects. SAP-TS offers high quality solutions to the SAP in a reasonable amount of computation time. SAP-TS results provide a level of detail that facilitates analysis of SAP instances as well as overall performance of the strategic airlift network. Insights into network bottlenecks, airlift shortfalls, and potential improvements in the airlift network infrastructure may be obtained by analyzing SAP-TS results.

Chapter 6 contains concluding remarks describing key contributions of this research and extensions of SAP-TS for future research. 


\section{Chapter 6: Conclusion}

This chapter outlines the major contributions of this dissertation and outlines improvements to the SAP-TS and extensions to the Strategic Airlift Problem.

\subsection{MAJOR CONTRIBUTIONS}

This research has made several contributions. These contributions are:

- the development of a flexible representation of the SAP for a TS approach

- the creation of an effective solution methodology for the SAP

- a proof of concept of a TS approach to the SAP

The SAP-TS solution representation captures key elements of the strategic airlift network using super arcs to represent routing of aircraft. This representation maintains sufficient fidelity of the SAP to create detailed schedules while avoiding the more cumbersome nodal approach. The SAP-TS solution representation also lends itself to the TS methodology. Key aspects of the SAP mission structure are captured and this structure is exploited in the development of SAP-TS neighborhoods.

The SAP-TS algorithm provides excellent solutions to the SAP. A series of neighborhoods specifically tailored to the SAP are dynamically selected during the conduct of the search based upon the phase of the search (mission assignment phase or mission improvement phase) and the search context (superdiversify, 
diversify or intensify). A flexible objective function was developed that can be easily adapted to target key aspects of the SAP. Weighting of feasibility (working MOG, fuel MOG, or UTE rate) or operational (undelivered cargo, late cargo) objective components enables aspects of SAP to be emphasized during the course of the search.

The efficacy of the SAP-TS algorithm was demonstrated using a series of operationally realistic scenarios. The SAP-TS results for these scenarios represented a significant improvement over AMOS results in a reasonable amount of time. These results also illustrate how the SAP-TS can be used to bridge the gap between lower quality (yet detailed) solutions obtained using simulation and the less detailed (yet higher quality) solutions obtained using large scale optimization such as integer or linear programming.

\subsection{FURTHER ENHANCEMENTS TO THE SAP-TS ALGORITHM}

Although SAP-TS obtains excellent results for the SAP, further research into several aspects of SAP-TS would lead to improved efficiency and effectiveness of the algorithm. Specifically, enhancements in the initial solution construction, mission assignment heuristic and partitioning of the mission assignment space would improve algorithm performance.

The SAP-TS initial solution construction heuristic ignores both MOG infeasibilities and UTE rate violations. The SAP-TS then strives to achieve feasibility during the conduct of the search. Starting the search with a near feasible solution may improve overall SAP-TS results. The additional time 
required to generate a near feasible initial solution may be well spent in achieving an improved starting solution for the search.

Several enhancements to the mission assignment heuristic warrant further study. Currently aircraft are assigned to loads based upon temporal constraints (aircraft availability) and load feasibility. Incorporating more sophisticated aircraft selection rules may improve aircraft mission loads. For example, it may be beneficial to incorporate metrics or rules that measure the "goodness" of a particular type of aircraft to load assignment based upon aircraft cargo preferences, percent fill of the aircraft and requirement priority. These and other rules could be adjusted to favor the assignment of particular aircraft types under different circumstances such as favoring the use of C17s over the older C5 fleet.

Another enhancement to the mission assignment heuristic centers around improving feasibility as the solution is constructed. Currently, as missions are created, arcs are selected out of set of possible arcs on a rotating basis. An improvement to this strategy would be to maintain a running tally of the fuel and working MOG status as missions are built. Arcs would then be selected based upon the MOG status along the arc for the time period of the current mission. In this way, the flow through the network would be dynamically adjusted as the solution is constructed yielding near feasible solutions.

A third enhancement to the mission assignment heuristic concerns the use of portions of the previous solution instead of a complete reconstruction of the solution. For example, for a BPS move, identify and update only the missions affected by swapping the two missions. The difficulty in this improvement lies in 
determining which missions are affected. Network constraints (MOG), temporal constraints (aircraft and requirement availability) and spatial constraints (route constraints) can cause missions other than the ones associated with the BPS move to be affected.

The final and perhaps most promising enhancement entails the use of a Group Theoretic TS (GTTS) approach to partition the search space during the mission assignment phase. As discussed in Section 2.3.4.4, GTTS has been shown by Wiley (2001) and Crino (2001) to be particularly effective in efficiently searching the solution space of portioning and ordering problems. During the mission assignment phase of SAP-TS, we could seek a good partitioning of the set of possible missions among the set of APOE-APOD pairs and a good ordering of the missions within APOE-APOD pairs. In this way, GTTS could be used to efficiently search the solution space during the mission assignment phase. The structure of neighborhoods used in the mission improvement phase neighborhoods does not readily lend itself to the use of GTTS.

\subsection{EXTENSIONS TO OTHER ASPECTS OF THE SAP}

Several extensions to the basic SAP investigated in this research would add to realism and encompass other aspects important to AMC analysts. Chief among these are the inclusion of transloading, theater constraints and more realistic modeling of the airlift network.

SAP-TS requirements have a known destination a priori. For the SAP, the destination is the APOD. However, during strategic airlift operations, transloading is frequently employed to stage requirements at or near the APODs 
so that requirements can be moved by other aircraft to their final destination in theater (which may or may not be the APOD). Typically this entails modeling the intra-theater airlift. Strategic assets deliver cargo to APODs and then intra-theater aircraft (usually smaller aircraft such as C130s and C141s) move requirements to their final destinations (usually to more austere bases) in theater. Modeling intratheater airlift is beyond the scope of this research. However, even in a strictly strategic sense, efficiencies may be gained by using some aircraft for the major legs from CONUS to "near" theater bases and other aircraft to shuttle from transload points to the APODs.

Operational plans often allocate aircraft to support a particular theater. An air unit's mission may support one or more theaters during the course of a deployment. Incorporating theater constraints imposes a partitioning of the aircraft which may be artificial. However, operational plans may require such a partitioning and thus research into the impact of this restriction would be beneficial.

Incorporating improvements that enhance realism in modeling the airlift network would provide additional insight into the SAP. In particular, adding the impact of weather, winds and seasonal temperatures can have a large impact on fuel considerations, timings along the route and aircraft loads.

\subsection{SUMMARY}

This research presented an effective solution methodology, the SAP-TS, which solves the SAP and obtains excellent solutions. The SAP-TS incorporates an efficient representation of the SAP that captures sufficient mission details for 
the construction of a detailed schedule yet remains flexible enough for application of the TS methodology. 


\section{Appendix A}

\section{SAP-TS Best Solution Scenario Mission Summaries}

\section{A.1 SCENARIO 1 SAP-TS MISSION SUMMARY}

\begin{tabular}{|c|c|c|c|c|c|c|c|c|c|}
\hline & & & & & \multicolumn{5}{|c|}{-------DELIVERED QUANTITIES--------' } \\
\hline Tail\# & Msn\# & REQT\# & APOE $\sim$ APOD & ARR APOD & OUT & OVER & BULK & PAX & TOTAL \\
\hline 0 & 1 & 47 & KNZY RJTY & 25.3723 & 0 & 0 & 0 & 309 & 61.8 \\
\hline 1 & 12 & 4 & KDNL OJ1X & 1.43128 & 0 & 0 & 0 & 233 & 46.6 \\
\hline 2 & 24 & 0 & KBLV LTAG & 0.86795 & 0 & 0 & 0 & 300 & 60 \\
\hline 2 & 25 & 54 & KNYL RJFU & 24.65088 & 0 & 0 & 0 & 286 & 57.2 \\
\hline 3 & 36 & 7 & KDSM LTAG & 2.17085 & 0 & 0 & 0 & 335 & 67 \\
\hline 3 & 37 & 54 & KNYL RJFU & 25.07701 & 0 & 0 & 0 & 252 & 50.4 \\
\hline 4 & 48 & 7 & KDSM LTAG & 1.87217 & 0 & 0 & 0 & 335 & 67 \\
\hline 4 & 49 & 54 & KNYL RJFU & 24.73459 & 0 & 0 & 0 & 335 & 67 \\
\hline 5 & 60 & 7 & KDSM LTAG & 2.47444 & 0 & 0 & 0 & 335 & 67 \\
\hline 5 & 61 & 54 & KNYL RJFU & 24.94374 & 0 & 0 & 0 & 335 & 67 \\
\hline 6 & 72 & 7 & KDSM LTAG & 3.95039 & 0 & 0 & 0 & 335 & 67 \\
\hline 6 & 73 & 54 & KNYL RJFU & 25.67685 & 0 & 0 & 0 & 22 & 4.4 \\
\hline 7 & 84 & 7 & KDSM LTAG & 2.31668 & 0 & 0 & 0 & 335 & 67 \\
\hline 8 & 96 & 7 & KDSM LTAG & 2.05439 & 0 & 0 & 0 & 25 & 5 \\
\hline 8 & 97 & 37 & KNLC $\sim \mathrm{RJCC}$ & 25.84286 & 0 & 0 & 0 & 335 & 67 \\
\hline 9 & 108 & 3 & KDMA $\sim \mathrm{OKBK}$ & 2.36032 & 0 & 0 & 0 & 335 & 67 \\
\hline 9 & 109 & 37 & KNLC $\sim \mathrm{RJCC}$ & 25.00862 & 0 & 0 & 0 & 335 & 67 \\
\hline 10 & 120 & 3 & KDMA OKBK & 1.92282 & 0 & 0 & 0 & 335 & 67 \\
\hline 10 & 121 & 37 & KNLC $\sim \mathrm{RJCC}$ & 25.85186 & 0 & 0 & 0 & 335 & 67 \\
\hline 21 & 252 & 3 & KDMA OKBK & 3.08948 & 0 & 0 & 0 & 335 & 67 \\
\hline 21 & 253 & 37 & KNLC $\sim \mathrm{RJCC}$ & 25.90129 & 0 & 0 & 0 & 260 & 52 \\
\hline 22 & 264 & 3 & KDMA OKBK & 2.06787 & 0 & 0 & 0 & 335 & 67 \\
\hline 22 & 265 & 37 & KNLC $\sim$ RJCC & 24.70233 & 0 & 0 & 0 & 325 & 65 \\
\hline 23 & 276 & 3 & KDMA OKBK & 1.92281 & 0 & 0 & 0 & 264 & 52.8 \\
\hline 24 & 289 & 55 & KNZC $\sim$ RJNN & 26.99249 & 0 & 0 & 0 & 18 & 3.6 \\
\hline 25 & 300 & 9 & ETAR LTAG & 1.5091 & 0 & 0 & 0 & 28 & 5.6 \\
\hline 25 & 301 & 58 & KDOV RJTY & 26.0375 & 0 & 0 & 0 & 123 & 24.6 \\
\hline 27 & 324 & 10 & KFSI LTAG & 6.65251 & 0 & 0 & 0 & 335 & 67 \\
\hline 28 & 336 & 10 & KFSI LTAG & 6.52556 & 0 & 0 & 0 & 6 & 1.2 \\
\hline 29 & 348 & 38 & KNTD RJCJ & 5.53394 & 0 & 0 & 0 & 233 & 46.6 \\
\hline 30 & 360 & 35 & KNKT $\sim$ RJAA & 5.08209 & 0 & 0 & 0 & 260 & 52 \\
\hline 41 & 492 & 35 & KNKT $\sim$ RJAA & 4.87706 & 0 & 0 & 0 & 62 & 12.4 \\
\hline 41 & 495 & 40 & KNUQ RKTC & 25.27149 & 0 & 0 & 0 & 89 & 17.8 \\
\hline 42 & 504 & 16 & KBLV LTBF & 5.66889 & 0 & 0 & 0 & 41 & 8.2 \\
\hline 43 & 516 & 15 & KGTB LTBA & 8.22802 & 0 & 0 & 0 & 195 & 39 \\
\hline 44 & 528 & 15 & KGTB LTBA & 8.23754 & 0 & 0 & 0 & 195 & 39 \\
\hline 45 & 540 & 15 & KGTB LTBA & 5.89444 & 0 & 0 & 0 & 52 & 10.4 \\
\hline 46 & 552 & 12 & KFWA LTAJ & 7.84795 & 0 & 0 & 0 & 195 & 39 \\
\hline 47 & 564 & 12 & KFWA LTAJ & 8.11845 & 0 & 0 & 0 & 195 & 39 \\
\hline 48 & 576 & 12 & KFWA LTAJ & 7.8353 & 0 & 0 & 0 & 195 & 39 \\
\hline 49 & 588 & 12 & KFWA LTAJ & 9.43053 & 0 & 0 & 0 & 58 & 11.6 \\
\hline 50 & 600 & 22 & KNGZ ETAR & 8.06274 & 0 & 0 & 0 & 195 & 39 \\
\hline 51 & 612 & 22 & KNGZ ETAR & 7.62524 & 0 & 0 & 0 & 195 & 39 \\
\hline 52 & 624 & 22 & KNGZ ETAR & 8.06247 & 0 & 0 & 0 & 8 & 1.6 \\
\hline
\end{tabular}




\begin{tabular}{|c|c|c|c|c|c|c|c|c|c|}
\hline \multirow[b]{2}{*}{ Tail\# } & \multirow[b]{2}{*}{ Msn\# } & \multirow[b]{2}{*}{ REQT\# } & \multirow[b]{2}{*}{ APOE $\sim$ APOD } & \multirow[b]{2}{*}{ ARR APOD } & \multicolumn{5}{|c|}{------DELIVERED QUANTITIES------- } \\
\hline & & & & & OUT & OVER & BULK & PAX & TOTAL \\
\hline 53 & 636 & 20 & KNGU LTCC & 8.10298 & 0 & 0 & 0 & 4 & 0.8 \\
\hline 54 & 648 & 18 & KNFW LTBU & 7.84703 & 0 & 0 & 0 & 2 & 0.4 \\
\hline 55 & 660 & 19 & KNGP LTBU & 9.90857 & 0 & 0 & 0 & 195 & 39 \\
\hline 57 & 684 & 32 & KSPS OKBK & 9.85513 & 0 & 0 & 0 & 12 & 2.4 \\
\hline 58 & 696 & 50 & KPDX RJNN & 11.60866 & 0 & 0 & 0 & 8 & 1.6 \\
\hline 59 & 708 & 30 & KSLC $\sim$ LTAN & 11.9553 & 0 & 0 & 0 & 195 & 39 \\
\hline 60 & 720 & 30 & KSLC $\sim$ LTAN & 12.34963 & 0 & 0 & 0 & 38 & 7.6 \\
\hline 61 & 732 & 29 & KSKF LTAG & 14.233 & 0 & 0 & 0 & 195 & 39 \\
\hline 62 & 744 & 29 & KSKF LTAG & 13.08586 & 0 & 0 & 0 & 195 & 39 \\
\hline 63 & 756 & 29 & KSKF LTAG & 14.67066 & 0 & 0 & 0 & 195 & 39 \\
\hline 64 & 768 & 29 & KSKF LTAG & 13.21942 & 0 & 0 & 0 & 195 & 39 \\
\hline 85 & 1020 & 29 & KSKF LTAG & 14.08717 & 0 & 0 & 0 & 195 & 39 \\
\hline 86 & 1032 & 29 & KSKF LTAG & 12.06503 & 0 & 0 & 0 & 195 & 39 \\
\hline 87 & 1044 & 29 & KSKF LTAG & 14.67066 & 0 & 0 & 0 & 195 & 39 \\
\hline 88 & 1056 & 29 & KSKF $\sim$ LAAG & 13.69337 & 0 & 0 & 0 & 195 & 39 \\
\hline 89 & 1068 & 29 & KSKF LTAG & 15.1078 & 0 & 0 & 0 & 44 & 8.8 \\
\hline 91 & 1092 & 36 & KNKX $\sim \mathrm{RJBB}$ & 12.74322 & 0 & 0 & 0 & 4 & 0.8 \\
\hline 92 & 1104 & 42 & KNXX RJNN & 15.2444 & 0 & 0 & 0 & 195 & 39 \\
\hline 93 & 1116 & 42 & KNXX RJNN & 14.69913 & 0 & 0 & 0 & 195 & 39 \\
\hline 94 & 1128 & 42 & KNXX RJNN & 14.73923 & 0 & 0 & 0 & 195 & 39 \\
\hline 95 & 1140 & 42 & KNXX RJNN & 15.36442 & 0 & 0 & 0 & 93 & 18.6 \\
\hline 96 & 1152 & 53 & KRCA RJBB & 15.08596 & 0 & 0 & 0 & 195 & 39 \\
\hline 97 & 1164 & 53 & KRCA RJBB & 14.96691 & 0 & 0 & 0 & 139 & 27.8 \\
\hline 98 & 1176 & 57 & KNZW RJSM & 15.72142 & 0 & 0 & 0 & 46 & 9.2 \\
\hline 99 & 1188 & 45 & KNZJ RJTA & 15.84363 & 0 & 0 & 0 & 120 & 24 \\
\hline 100 & 1200 & 39 & KNTU RJSM & 15.75544 & 0 & 0 & 0 & 123 & 24.6 \\
\hline 101 & 1212 & 34 & KSTJ OMAD & 22.859 & 0 & 0 & 0 & 133 & 26.6 \\
\hline 102 & 1224 & 51 & KPOB RJTY & 23.71979 & 0 & 0 & 0 & 123 & 24.6 \\
\hline 103 & 1236 & 49 & KPBG $\sim$ RJFU & 26.11948 & 0 & 0 & 0 & 195 & 39 \\
\hline 104 & 1248 & 49 & KPBG RJFU & 25.60014 & 0 & 0 & 0 & 195 & 39 \\
\hline 105 & 1260 & 49 & KPBG RJFU & 25.21156 & 0 & 0 & 0 & 195 & 39 \\
\hline 106 & 1272 & 49 & KPBG $\sim$ RJFU & 26.26698 & 0 & 0 & 0 & 195 & 39 \\
\hline 107 & 1284 & 49 & KPBG $\sim$ RJFU & 26.53825 & 0 & 0 & 0 & 195 & 39 \\
\hline 108 & 1296 & 49 & KPBG RJFU & 26.98131 & 0 & 0 & 0 & 195 & 39 \\
\hline 109 & 1308 & 49 & KPBG RJFU & 25.97322 & 0 & 0 & 0 & 60 & 12 \\
\hline 135 & 1620 & 6 & KDPG OJHF & 1.54012 & 0 & 0 & 87.4 & 0 & 87.4 \\
\hline 135 & 1621 & 30 & KSLC $\sim$ LTAN & 11.94681 & 0 & 0 & 87.4 & 0 & 87.4 \\
\hline 136 & 1632 & 6 & KDPG OJHF & 1.55101 & 0 & 0 & 87.4 & 0 & 87.4 \\
\hline 136 & 1633 & 30 & KSLC $\sim$ LTAN & 12.03769 & 0 & 0 & 87.4 & 0 & 87.4 \\
\hline 137 & 1644 & 6 & KDPG OJHF & 1.68563 & 0 & 0 & 87.4 & 0 & 87.4 \\
\hline 137 & 1645 & 29 & KSKF LTAG & 12.15363 & 0 & 0 & 87.4 & 0 & 87.4 \\
\hline 138 & 1656 & 6 & KDPG OJHF & 1.68797 & 0 & 0 & 38.4 & 0 & 38.4 \\
\hline 138 & 1657 & 29 & KSKF LTAG & 11.8504 & 0 & 0 & 87.4 & 0 & 87.4 \\
\hline 139 & 1668 & 4 & KDNL OJ1X & 1.34813 & 0 & 0 & 87.4 & 0 & 87.4 \\
\hline 139 & 1669 & 29 & KSKF LTAG & 11.85732 & 0 & 0 & 87.4 & 0 & 87.4 \\
\hline 140 & 1680 & 4 & KDNL OJ1X & 0.91101 & 0 & 0 & 87.4 & 0 & 87.4 \\
\hline 140 & 1681 & 29 & KSKF LTAG & 14.87635 & 0 & 0 & 87.4 & 0 & 87.4 \\
\hline 141 & 1692 & 4 & KDNL OJ1X & 1.06376 & 0 & 0 & 87.4 & 0 & 87.4 \\
\hline 141 & 1693 & 29 & KSKF $\sim$ LTAG & 12.15363 & 0 & 0 & 87.4 & 0 & 87.4 \\
\hline 142 & 1705 & 29 & KSKF LTAG & 14.14718 & 0 & 0 & 18.4 & 0 & 18.4 \\
\hline 143 & 1716 & 1 & KDLF $\sim$ LTAG & 1.33576 & 0 & 0 & 23.5 & 0 & 23.5 \\
\hline 143 & 1717 & 27 & KSGH OJHF & 11.93983 & 0 & 0 & 72.2 & 0 & 72.2 \\
\hline 144 & 1728 & 3 & KDMA OKBK & 2.11025 & 0 & 0 & 87.4 & 0 & 87.4 \\
\hline 144 & 1729 & 27 & KSGH OJHF & 11.75546 & 0 & 0 & 72.2 & 0 & 72.2 \\
\hline 145 & 1740 & 3 & KDMA OKBK & 2.10945 & 0 & 0 & 87.4 & 0 & 87.4 \\
\hline 145 & 1741 & 27 & KSGH OJHF & 12.53198 & 0 & 0 & 72.2 & 0 & 72.2 \\
\hline 146 & 1752 & 3 & KDMA OKBK & 2.25529 & 0 & 0 & 87.4 & 0 & 87.4 \\
\hline 146 & 1753 & 27 & KSGH OJHF & 13.39577 & 0 & 0 & 72.2 & 0 & 72.2 \\
\hline
\end{tabular}




\begin{tabular}{|c|c|c|c|c|c|c|c|c|c|}
\hline & & & & & \multicolumn{5}{|c|}{------DELIVERED QUANTITIES------- } \\
\hline Tail\# & Msn\# & REQT\# & APOE $\sim$ APOD & ARR APOD & OUT & OVER & BULK & PAX & TOTAL \\
\hline 147 & 1764 & 3 & KDMA OKBK & 2.98525 & 0 & 0 & 87.4 & 0 & 87.4 \\
\hline 147 & 1765 & 27 & KSGH OJHF & 12.19504 & 0 & 0 & 72.2 & 0 & 72.2 \\
\hline 148 & 1776 & 3 & KDMA $\sim \mathrm{OKBK}$ & 2.83862 & 0 & 0 & 87.4 & 0 & 87.4 \\
\hline 148 & 1777 & 27 & $\mathrm{KSGH} \sim \mathrm{OJHF}$ & 11.90129 & 0 & 0 & 72.2 & 0 & 72.2 \\
\hline 149 & 1788 & 3 & KDMA $\sim \mathrm{OKBK}$ & 1.96431 & 0 & 0 & 18.4 & 0 & 18.4 \\
\hline 149 & 1789 & 27 & KSGH OJHF & 12.82364 & 0 & 0 & 72.2 & 0 & 72.2 \\
\hline 150 & 1800 & 9 & ETAR LTAG & 1.55073 & 0 & 0 & 45.5 & 0 & 45.5 \\
\hline 150 & 1801 & 27 & KSGH OJHF & 13.24993 & 0 & 0 & 72.2 & 0 & 72.2 \\
\hline 151 & 1812 & 10 & KFSI LTAG & 6.54819 & 0 & 0 & 10.2 & 0 & 10.2 \\
\hline 151 & 1813 & 27 & KSGH OJHF & 12.19504 & 0 & 0 & 72.2 & 0 & 72.2 \\
\hline 152 & 1824 & 38 & KNTD RJCJ & 6.22758 & 0 & 0 & 87.4 & 0 & 87.4 \\
\hline 152 & 1825 & 27 & KSGH OJHF & 11.75546 & 0 & 0 & 72.2 & 0 & 72.2 \\
\hline 153 & 1836 & 38 & KNTD RJCJ & 6.16659 & 0 & 0 & 87.4 & 0 & 87.4 \\
\hline 153 & 1837 & 27 & KSGH OJHF & 12.53197 & 0 & 0 & 65.8 & 0 & 65.8 \\
\hline 154 & 1848 & 38 & KNTD RJCJ & 6.6839 & 0 & 0 & 65.53 & 0 & 65.53 \\
\hline 154 & 1849 & 36 & KNKX $\sim$ RJBB & 12.97403 & 0 & 0 & 72.2 & 0 & 72.2 \\
\hline 182 & 2184 & 38 & KNTD RJCJ & 6.35995 & 0 & 0 & 39.67 & 0 & 39.67 \\
\hline 182 & 2185 & 36 & KNKX RJBB & 12.90567 & 0 & 0 & 18.2 & 0 & 18.2 \\
\hline 183 & 2196 & 14 & KGSP $\sim$ LAZ & 5.75363 & 0 & 0 & 34.5 & 0 & 34.5 \\
\hline 183 & 2197 & 31 & KSPI LTAG & 15.05318 & 0 & 0 & 87.4 & 0 & 87.4 \\
\hline 184 & 2208 & 12 & KFWA LTAJ & 9.3673 & 0 & 0 & 72.2 & 0 & 72.2 \\
\hline 184 & 2209 & 31 & KSPI LTAG & 22.19201 & 0 & 0 & 87.4 & 0 & 87.4 \\
\hline 185 & 2220 & 12 & KFWA LTAJ & 9.63838 & 0 & 0 & 72.2 & 0 & 72.2 \\
\hline 185 & 2221 & 31 & KSPI LTAG & 22.06512 & 0 & 0 & 87.4 & 0 & 87.4 \\
\hline 186 & 2232 & 12 & KFWA LTAJ & 8.37063 & 0 & 0 & 72.2 & 0 & 72.2 \\
\hline 186 & 2233 & 31 & KSPI LTAG & 22.04623 & 0 & 0 & 60.1 & 0 & 60.1 \\
\hline 187 & 2244 & 12 & KFWA LTAJ & 8.79964 & 0 & 0 & 72.2 & 0 & 72.2 \\
\hline 187 & 2245 & 53 & KRCA RJBB & 15.19035 & 0 & 0 & 56.9 & 0 & 56.9 \\
\hline 188 & 2256 & 12 & KFWA LTAJ & 8.63813 & 0 & 0 & 72.2 & 0 & 72.2 \\
\hline 188 & 2257 & 57 & KNZW RJSM & 15.81964 & 0 & 0 & 56 & 0 & 56 \\
\hline 189 & 2268 & 12 & KFWA $\sim$ TAJ & 8.32588 & 0 & 0 & 72.2 & 0 & 72.2 \\
\hline 189 & 2269 & 39 & KNTU RJSM & 15.93689 & 0 & 0 & 5.6 & 0 & 5.6 \\
\hline 190 & 2280 & 12 & KFWA LTAJ & 8.07888 & 0 & 0 & 22.1 & 0 & 22.1 \\
\hline 190 & 2281 & 46 & KNZW RJTT & 17.14444 & 0 & 0 & 6.2 & 0 & 6.2 \\
\hline 191 & 2292 & 20 & KNGU LTCC & 8.03112 & 0 & 0 & 82.7 & 0 & 82.7 \\
\hline 191 & 2293 & 44 & KNZC $\sim$ RJSM & 20.8154 & 0 & 0 & 8.9 & 0 & 8.9 \\
\hline 192 & 2304 & 20 & KNGU LTCC & 7.72749 & 0 & 0 & 7.7 & 0 & 7.7 \\
\hline 192 & 2305 & 37 & KNLC $\sim \mathrm{RJCC}$ & 25.44674 & 0 & 0 & 87.4 & 0 & 87.4 \\
\hline 193 & 2316 & 18 & KNFW LTBU & 7.78595 & 0 & 0 & 20.3 & 0 & 20.3 \\
\hline 193 & 2317 & 37 & KNLC $\sim$ RJCC & 26.07103 & 0 & 0 & 87.4 & 0 & 87.4 \\
\hline 194 & 2328 & 24 & KBLV ETAR & 9.4791 & 0 & 0 & 8.9 & 0 & 8.9 \\
\hline 194 & 2329 & 37 & KNLC $\sim$ RJCC & 25.66004 & 0 & 0 & 87.4 & 0 & 87.4 \\
\hline 195 & 2341 & 37 & KNLC $\sim$ RJCC & 26.67198 & 0 & 0 & 65.53 & 0 & 65.53 \\
\hline 196 & 2352 & 33 & KSSC OMAA & 12.68375 & 0 & 0 & 7.9 & 0 & 7.9 \\
\hline 196 & 2353 & 37 & KNLC $\sim$ RJCC & 25.29804 & 0 & 0 & 78.66 & 0 & 78.66 \\
\hline 212 & 2544 & 5 & KDOV $\sim \mathrm{OJ} 2 \mathrm{X}$ & 0.98825 & 0 & 30.9 & 0 & 5 & 31.9 \\
\hline 212 & 2545 & 28 & KSKA LTAG & 13.78139 & 0 & 6.7 & 0 & 7 & 8.1 \\
\hline 213 & 2556 & 4 & KDNL OJ1X & 1.23157 & 13 & 6 & 17.8 & 0 & 36.8 \\
\hline 213 & 2557 & 31 & KSPI LTAG & 22.17242 & 0 & 33.6 & 0 & 0 & 33.6 \\
\hline 214 & 2568 & 2 & KDLH LTAG & 1.88993 & 0 & 18.1 & 0 & 0 & 18.1 \\
\hline 214 & 2569 & 40 & KNUQ RKTC & 26.18085 & 18 & 0 & 0 & 0 & 18 \\
\hline 215 & 2580 & 1 & KDLF LTAG & 1.84806 & 33.2 & 0 & 0 & 0 & 33.2 \\
\hline 215 & 2581 & 53 & KRCA RJBB & 14.84693 & 19.2 & 23.1 & 0 & 0 & 42.3 \\
\hline 216 & 2592 & 0 & KBLV LTAG & 1.71172 & 0 & 33.5 & 0 & 0 & 33.5 \\
\hline 216 & 2593 & 57 & KNZW RJSM & 16.57491 & 0 & 20.9 & 0 & 0 & 20.9 \\
\hline 217 & 2604 & 8 & KDYS LTAG & 4.01011 & 37.2 & 0 & 0 & 0 & 37.2 \\
\hline 217 & 2605 & 45 & KNZJ RJTA & 15.83203 & 0 & 44.5 & 0 & 0 & 44.5 \\
\hline 218 & 2616 & 7 & KDSM LTAG & 4.23208 & 0 & 6.6 & 0 & 0 & 6.6 \\
\hline
\end{tabular}




\begin{tabular}{|c|c|c|c|c|c|c|c|c|c|}
\hline & & & & & -------D & LIVEREI & QUANTI' & ES----.- & \\
\hline Tail\# & Msn\# & REQT\# & $\begin{array}{l}\text { APOE } \sim \text { APOD } \\
\end{array}$ & ARR APOD & OUT & OVER & BULK & PAX & TOTAL \\
\hline 218 & 2617 & 39 & KNTU RJSM & 16.56 & 6.5 & 6.7 & 0 & 0 & 13.2 \\
\hline 219 & 2628 & 25 & KWRI $\sim$ ETAR & 1.52107 & 0 & 0 & 0 & 106 & 21.2 \\
\hline 219 & 2629 & 46 & KNZW RJTT & 16.96273 & 58.1 & 0 & 0 & 0 & 58.1 \\
\hline 220 & 2641 & 46 & KNZW RJTT & 17.52428 & 32.2 & 0 & 0 & 0 & 32.2 \\
\hline 220 & 2640 & 40 & KNUQ RKTC & 26.18302 & 18 & 0 & 0 & 0 & 18 \\
\hline 221 & 2652 & 11 & KFSM $\sim$ LTAI & 5.06488 & 16.5 & 53.97 & 0 & 0 & 70.47 \\
\hline 221 & 2653 & 52 & KPSM $\sim$ RJAA & 18.24575 & 34.5 & 0 & 0 & 0 & 34.5 \\
\hline 227 & 2724 & 25 & KWRI ETAR & 8.83547 & 0 & 14.5 & 0 & 14 & 17.3 \\
\hline 227 & 2725 & 19 & KNGP $\sim$ LTBU & 11.19433 & 51.39 & 0 & 0 & 62 & 63.79 \\
\hline 233 & 2796 & 11 & KFSM $\sim$ LTAI & 5.03734 & 0 & 44.63 & 0 & 3 & 45.23 \\
\hline 233 & 2797 & 52 & KPSM RJAA & 18.55087 & 33.71 & 0 & 0 & 0 & 33.71 \\
\hline 234 & 2808 & 38 & KNTD RJCJ & 5.9667 & 23.3 & 0 & 0 & 0 & 23.3 \\
\hline 234 & 2809 & 52 & KPSM RJAA & 18.11437 & 34.5 & 0 & 0 & 0 & 34.5 \\
\hline 235 & 2820 & 35 & KNKT $\sim$ RJAA & 5.53619 & 35.53 & 0 & 0 & 0 & 35.53 \\
\hline 235 & 2821 & 52 & KPSM $\sim$ RJAA & 17.9582 & 34.5 & 0 & 0 & 0 & 34.5 \\
\hline 236 & 2832 & 35 & KNKT RJAA & 5.18676 & 41.3 & 0 & 0 & 0 & 41.3 \\
\hline 236 & 2833 & 52 & KPSM $\sim$ RJAA & 18.52429 & 34.5 & 0 & 0 & 0 & 34.5 \\
\hline 237 & 2844 & 35 & KNKT $\sim$ RJAA & 5.1764 & 41.3 & 0 & 0 & 0 & 41.3 \\
\hline 237 & 2845 & 52 & KPSM $\sim$ RJAA & 18.24575 & 34.5 & 0 & 0 & 0 & 34.5 \\
\hline 238 & 2856 & 35 & KNKT RJAA & 5.50964 & 41.3 & 0 & 0 & 0 & 41.3 \\
\hline 238 & 2857 & 40 & KNUQ RKTC & 25.8384 & 18 & 0 & 0 & 0 & 18 \\
\hline 239 & 2868 & 35 & KNKT $\sim$ RJAA & 5.304 & 41.3 & 0 & 0 & 0 & 41.3 \\
\hline 239 & 2869 & 44 & KNZC $\sim$ RJSM & 20.93269 & 0 & 10.5 & 0 & 0 & 10.5 \\
\hline 240 & 2880 & 35 & KNKT $\sim$ RJAA & 5.53619 & 35.53 & 0 & 0 & 0 & 35.53 \\
\hline 240 & 2881 & 48 & KOAK RODN & 22.28878 & 18 & 0 & 0 & 0 & 18 \\
\hline 241 & 2892 & 35 & KNKT RJAA & 5.22315 & 14.33 & 0 & 0 & 0 & 14.33 \\
\hline 241 & 2893 & 34 & KSTJ $\sim$ OMAD & 23.22109 & 0 & 14.2 & 0 & 0 & 14.2 \\
\hline 242 & 2904 & 16 & KBLV LTBF & 6.02388 & 5.6 & 6.7 & 0 & 0 & 12.3 \\
\hline 242 & 2905 & 40 & KNUQ $\sim$ RKTC & 25.83634 & 18 & 0 & 0 & 0 & 18 \\
\hline 250 & 3000 & 15 & KGTB LTBA & 7.99828 & 0 & 62 & 0 & 0 & 62 \\
\hline 250 & 3001 & 40 & KNUQ RKTC & 26.07979 & 23.7 & 0 & 0 & 0 & 23.7 \\
\hline 251 & 3012 & 15 & KGTB LTBA & 7.9592 & 0 & 26.8 & 0 & 0 & 26.8 \\
\hline 251 & 3013 & 40 & KNUQ RKTC & 24.79895 & 23.9 & 0 & 0 & 0 & 23.9 \\
\hline 252 & 3024 & 21 & KNGU LTAN & 8.26226 & 0 & 47.9 & 0 & 0 & 47.9 \\
\hline 252 & 3025 & 40 & KNUQ RKTC & 25.70268 & 23.7 & 0 & 0 & 0 & 23.7 \\
\hline 253 & 3036 & 21 & KNGU LTAN & 8.37494 & 0 & 47.9 & 0 & 0 & 47.9 \\
\hline 253 & 3037 & 40 & KNUQ RKTC & 26.05527 & 23.7 & 0 & 0 & 0 & 23.7 \\
\hline 254 & 3048 & 21 & KNGU LTAN & 8.40497 & 0 & 14.6 & 0 & 0 & 14.6 \\
\hline 254 & 3049 & 40 & KNUQ RKTC & 25.71999 & 23.7 & 0 & 0 & 0 & 23.7 \\
\hline 255 & 3060 & 17 & KBLV OKBK & 7.98359 & 0 & 31.4 & 0 & 0 & 31.4 \\
\hline 255 & 3061 & 40 & KNUQ $\sim$ RKTC & 25.27782 & 23.7 & 0 & 0 & 0 & 23.7 \\
\hline 256 & 3072 & 17 & KBLV OKBK & 7.98391 & 0 & 31.4 & 0 & 0 & 31.4 \\
\hline 256 & 3073 & 40 & KNUQ RKTC & 26.12376 & 23.7 & 0 & 0 & 0 & 23.7 \\
\hline 257 & 3084 & 17 & KBLV OKBK & 7.98274 & 0 & 13.1 & 0 & 0 & 13.1 \\
\hline 257 & 3085 & 40 & KNUQ RKTC & 25.56334 & 23.7 & 0 & 0 & 0 & 23.7 \\
\hline 258 & 3096 & 13 & ETAR LTAG & 7.24411 & 22.3 & 0 & 0 & 0 & 22.3 \\
\hline 258 & 3097 & 40 & KNUQ RKTC & 25.78812 & 23.7 & 0 & 0 & 0 & 23.7 \\
\hline 259 & 3108 & 23 & KNHK $\sim$ ETAR & 9.55477 & 32 & 0 & 0 & 0 & 32 \\
\hline 259 & 3109 & 52 & KPSM RJAA & 18.33849 & 23.99 & 0 & 0 & 0 & 23.99 \\
\hline 260 & 3121 & 40 & KNUQ RKTC & 26.19576 & 23.7 & 0 & 0 & 0 & 23.7 \\
\hline 261 & 3132 & 19 & KNGP $\sim$ LTBU & 11.07868 & 50.5 & 0 & 0 & 17 & 53.9 \\
\hline 261 & 3133 & 40 & KNUQ RKTC & 25.99033 & 23.7 & 0 & 0 & 0 & 23.7 \\
\hline 262 & 3144 & 19 & KNGP LTBU & 10.13311 & 50.5 & 0 & 0 & 17 & 53.9 \\
\hline 262 & 3145 & 53 & KRCA RJBB & 14.83351 & 26.2 & 11.5 & 0 & 0 & 37.7 \\
\hline 263 & 3156 & 19 & KNGP LTBU & 11.04319 & 50.5 & 0 & 0 & 17 & 53.9 \\
\hline 263 & 3157 & 40 & KNUQ RKTC & 25.60885 & 23.7 & 0 & 0 & 0 & 23.7 \\
\hline 264 & 3168 & 19 & KNGP $\sim$ LTBU & 9.91228 & 50.5 & 0 & 0 & 14 & 53.3 \\
\hline 264 & 3169 & 40 & KNUQ $\sim$ RKTC & 25.12201 & 23.7 & 0 & 0 & 0 & 23.7 \\
\hline
\end{tabular}




\begin{tabular}{|c|c|c|c|c|c|c|c|c|c|}
\hline & & & & & \multicolumn{5}{|c|}{--------DELIVERED QUANTITIES-------- } \\
\hline Tail\# & Msn\# & REQT\# & APOE $\sim$ APOD & ARR APOD & OUT & OVER & BULK & PAX & TOTAL \\
\hline 265 & 3181 & 9 & ETAR LTAG & 1.53577 & 0.5 & 11.2 & 0 & 0 & 11.7 \\
\hline 265 & 3180 & 19 & KNGP LTBU & 10.75323 & 50.5 & 0 & 0 & 0 & 50.5 \\
\hline 266 & 3192 & 19 & KNGP LTBU & 10.13121 & 50.5 & 0 & 0 & 0 & 50.5 \\
\hline 266 & 3193 & 40 & KNUQ RKTC & 24.9749 & 23.7 & 0 & 0 & 0 & 23.7 \\
\hline 267 & 3204 & 19 & KNGP $\sim$ LTBU & 10.787 & 50.5 & 0 & 0 & 0 & 50.5 \\
\hline 267 & 3205 & 37 & KNLC $\sim$ RJCC & 25.19839 & 0 & 0 & 41.4 & 0 & 41.4 \\
\hline 268 & 3216 & 19 & KNGP $\sim$ LTBU & 11.00809 & 45.71 & 0 & 0 & 0 & 45.71 \\
\hline 268 & 3217 & 37 & KNLC $\sim$ RJCC & 24.85889 & 0 & 0 & 7.62 & 14 & 10.42 \\
\hline 269 & 3229 & 56 & KNZJ RJOI & 26.85406 & 0 & 24.5 & 20 & 0 & 44.5 \\
\hline 270 & 3240 & 24 & KBLV ETAR & 9.61163 & 0 & 10.5 & 0 & 0 & 10.5 \\
\hline 270 & 3241 & 58 & KDOV RJTY & 26.31932 & 34 & 0 & 0 & 0 & 34 \\
\hline 271 & 3252 & 26 & KWRI ETAR & 9.5546 & 26.2 & 0 & 1.2 & 0 & 27.4 \\
\hline 272 & 3264 & 26 & KWRI ETAR & 9.43654 & 26.2 & 0 & 0 & 0 & 26.2 \\
\hline 273 & 3276 & 26 & KWRI ETAR & 9.43657 & 26.2 & 0 & 0 & 0 & 26.2 \\
\hline 274 & 3288 & 26 & KWRI ETAR & 9.55455 & 11.7 & 0 & 0 & 0 & 11.7 \\
\hline 275 & 3300 & 43 & KNYL RJOI & 12.13939 & 16.8 & 0 & 0 & 0 & 16.8 \\
\hline 276 & 3312 & 43 & KNYL $\sim$ RJOI & 11.93732 & 15.2 & 0 & 0 & 0 & 15.2 \\
\hline 277 & 3324 & 41 & KNUW RKTD & 12.0323 & 0 & 59.6 & 0 & 0 & 59.6 \\
\hline 278 & 3336 & 41 & KNUW RKTD & 12.73392 & 0 & 59.6 & 0 & 0 & 59.6 \\
\hline 279 & 3348 & 41 & KNUW $\sim$ RKTD & 11.88505 & 0 & 59.6 & 0 & 0 & 59.6 \\
\hline 280 & 3360 & 41 & KNUW RKTD & 12.55906 & 0 & 47.93 & 0 & 0 & 47.93 \\
\hline 281 & 3372 & 41 & KNUW RKTD & 12.76434 & 0 & 47.93 & 0 & 0 & 47.93 \\
\hline 282 & 3384 & 41 & KNUW RKTD & 11.86462 & 0 & 35.75 & 0 & 0 & 35.75 \\
\hline 283 & 3396 & 33 & $\mathrm{KSSC} \sim \mathrm{OMAA}$ & 12.15142 & 51.4 & 0 & 0 & 0 & 51.4 \\
\hline 284 & 3408 & 33 & $\mathrm{KSSC} \sim \mathrm{OMAA}$ & 12.28297 & 51.4 & 0 & 0 & 0 & 51.4 \\
\hline 285 & 3420 & 33 & KSSC $\sim \mathrm{OMAA}$ & 13.01233 & 51.4 & 0 & 0 & 0 & 51.4 \\
\hline 286 & 3432 & 33 & $\mathrm{KSSC} \sim \mathrm{OMAA}$ & 13.01276 & 44.6 & 0 & 0 & 0 & 44.6 \\
\hline 287 & 3444 & 30 & KSLC $\sim$ LTAN & 12.81735 & 1.2 & 33.6 & 28.02 & 0 & 62.82 \\
\hline 288 & 3456 & 30 & KSLC $\sim$ LTAN & 12.99006 & 0 & 0 & 41.4 & 0 & 41.4 \\
\hline 289 & 3468 & 30 & KSLC $\sim$ LTAN & 12.99246 & 0 & 0 & 35.78 & 0 & 35.78 \\
\hline
\end{tabular}

Table A.1 Scenario 1 Mission Summary

\section{A.2 SCENARIO 2 SAP-TS MISSION SUMMARY}

\begin{tabular}{|c|c|c|c|c|c|c|c|c|c|}
\hline \multirow[b]{2}{*}{ Tail\# } & \multirow[b]{2}{*}{ Msn\# } & \multirow[b]{2}{*}{ REQT\# } & \multirow[b]{2}{*}{ APOE APOD } & \multirow[b]{2}{*}{ ARR APOD } & \multicolumn{5}{|c|}{-------DELIVERED QUANTITIES-------- } \\
\hline & & & & & OUT & OVER & BULK & PAX & TOTAL \\
\hline 0 & 0 & 2 & KGRK OKBK & 1.07281 & 0 & 0 & 0 & 335 & 67 \\
\hline 0 & 2 & 54 & KBIF $\sim \mathrm{OBBS}$ & 5.74413 & 0 & 0 & 0 & 70 & 14 \\
\hline 0 & 2 & 33 & KBIF OBBS & 5.74413 & 0 & 0 & 0 & 70 & 14 \\
\hline 1 & 12 & 2 & KGRK $\sim \mathrm{OKBK}$ & 1.06135 & 0 & 0 & 0 & 335 & 67 \\
\hline 1 & 13 & 41 & KMUO OOTH & 3.97048 & 0 & 0 & 0 & 28 & 5.6 \\
\hline 2 & 24 & 2 & KGRK $\sim \mathrm{OKBK}$ & 1.0561 & 0 & 0 & 0 & 81 & 16.2 \\
\hline 2 & 25 & 34 & $\mathrm{KGRK} \sim \mathrm{OKBK}$ & 3.87702 & 0 & 0 & 0 & 335 & 67 \\
\hline 2 & 27 & 60 & KBIF $\sim \mathrm{OBBS}$ & 8.55218 & 0 & 0 & 0 & 124 & 24.8 \\
\hline 3 & 36 & 1 & KBIF $\sim \mathrm{OKBK}$ & 1.10818 & 0 & 0 & 0 & 229 & 45.8 \\
\hline 3 & 39 & 110 & KGRK $\sim \mathrm{OKBK}$ & 9.87685 & 0 & 0 & 0 & 146 & 29.2 \\
\hline 4 & 48 & 22 & KMGE OKBK & 2.82176 & 0 & 0 & 0 & 176 & 35.2 \\
\hline 5 & 60 & 3 & $\mathrm{KDOV} \sim \mathrm{OKBK}$ & 3.7705 & 0 & 0 & 0 & 63 & 12.6 \\
\hline 5 & 60 & 42 & KDOV OKBK & 3.7705 & 0 & 0 & 0 & 45 & 9 \\
\hline 5 & 61 & 31 & KPOB OEKJ & 6.79366 & 0 & 0 & 0 & 28 & 5.6 \\
\hline 5 & 61 & 76 & KPOB OEKJ & 6.79366 & 0 & 0 & 0 & 54 & 10.8 \\
\hline 5 & 62 & 56 & KTIK OEKJ & 9.11462 & 0 & 0 & 0 & 108 & 21.6 \\
\hline 5 & 62 & 40 & KTIK $\sim \mathrm{OEKJ}$ & 9.11462 & 0 & 0 & 0 & 50 & 10 \\
\hline 6 & 74 & 71 & KGRK $\sim \mathrm{OKBK}$ & 6.87178 & 0 & 0 & 0 & 276 & 55.2 \\
\hline
\end{tabular}




\begin{tabular}{|c|c|c|c|c|c|c|c|c|c|}
\hline \multirow[b]{2}{*}{ Tail\# } & \multirow[b]{2}{*}{ Msn\# } & \multirow[b]{2}{*}{ REQT\# } & \multirow[b]{2}{*}{ APOE $\sim$ APOD } & \multirow[b]{2}{*}{ ARR APOD } & \multicolumn{5}{|c|}{-------DELIVERED QUANTITIES-------- } \\
\hline & & & & & OUT & OVER & BULK & PAX & TOTAL \\
\hline 6 & 74 & 79 & KGRK $\sim \mathrm{OKBK}$ & 6.87178 & 0 & 0 & 0 & 16 & 3.2 \\
\hline 7 & 86 & 131 & KGRK OKBK & 10.87178 & 0 & 0 & 0 & 335 & 67 \\
\hline 7 & 87 & 131 & $\mathrm{KGRK} \sim \mathrm{OKBK}$ & 13.22322 & 0 & 0 & 0 & 196 & 39.2 \\
\hline 8 & 97 & 29 & KDOV OOTH & 4.33611 & 0 & 0 & 0 & 25 & 5 \\
\hline 8 & 98 & 128 & KMGE OKBK & 10.82171 & 0 & 0 & 0 & 15 & 3 \\
\hline 8 & 98 & 72 & KMGE OKBK & 10.82171 & 0 & 0 & 0 & 33 & 6.6 \\
\hline 9 & 108 & 10 & KGRK $\sim \mathrm{OKBK}$ & 1.99356 & 0 & 0 & 0 & 335 & 67 \\
\hline 9 & 108 & 11 & KGRK $\sim \mathrm{OKBK}$ & 1.99356 & 0 & 0 & 0 & 18 & 3.6 \\
\hline 9 & 108 & 12 & KGRK $\sim \mathrm{OKBK}$ & 1.99356 & 0 & 0 & 0 & 6 & 1.2 \\
\hline 10 & 121 & 24 & KBIF $\sim \mathrm{OEKJ}$ & 4.6022 & 0 & 0 & 46.3 & 0 & 46.3 \\
\hline 10 & 122 & 48 & KMUO OTBD & 7.03701 & 0 & 0 & 54.2 & 0 & 54.2 \\
\hline 10 & 123 & 86 & KRIV OBBS & 9.50734 & 0 & 0 & 81.1 & 0 & 81.1 \\
\hline 10 & 124 & 112 & KDOV OKBK & 11.95085 & 0 & 0 & 4.7 & 0 & 4.7 \\
\hline 10 & 124 & 120 & KDOV $\sim \mathrm{OKBK}$ & 11.95085 & 0 & 0 & 21.7 & 0 & 21.7 \\
\hline 10 & 124 & 124 & KDOV OKBK & 11.95085 & 0 & 0 & 23.8 & 0 & 23.8 \\
\hline 11 & 132 & 6 & KSKF $\sim \mathrm{OKBK}$ & 2.11152 & 0 & 0 & 44.6 & 0 & 44.6 \\
\hline 11 & 133 & 24 & KBIF $\sim \mathrm{OEKJ}$ & 4.55727 & 0 & 0 & 87.4 & 0 & 87.4 \\
\hline 11 & 134 & 48 & KMUO OTBD & 6.98475 & 0 & 0 & 72.2 & 0 & 72.2 \\
\hline 11 & 136 & 112 & KDOV OKBK & 9.8755 & 0 & 0 & 87.4 & 0 & 87.4 \\
\hline 12 & 145 & 23 & KDOV OKBK & 4.51399 & 0 & 0 & 70.4 & 0 & 70.4 \\
\hline 12 & 145 & 26 & KDOV OKBK & 4.51399 & 0 & 0 & 25.45 & 0 & 25.45 \\
\hline 12 & 146 & 48 & KMUO OTBD & 6.96796 & 0 & 0 & 72.2 & 0 & 72.2 \\
\hline 12 & 148 & 126 & KDOV OBBS & 9.83534 & 0 & 0 & 18.9 & 0 & 18.9 \\
\hline 13 & 156 & 10 & KGRK $\sim \mathrm{OKBK}$ & 2.15586 & 0 & 0 & 59.7 & 0 & 59.7 \\
\hline 13 & 156 & 11 & KGRK $\sim \mathrm{OKBK}$ & 2.15586 & 0 & 0 & 8.5 & 0 & 8.5 \\
\hline 13 & 156 & 12 & KGRK $\sim \mathrm{OKBK}$ & 2.15586 & 0 & 0 & 5.6 & 0 & 5.6 \\
\hline 13 & 157 & 26 & KDOV OKBK & 4.57607 & 0 & 0 & 87.4 & 0 & 87.4 \\
\hline 13 & 159 & 35 & KWRI OKBK & 7.00967 & 0 & 0 & 65.41 & 0 & 65.41 \\
\hline 14 & 171 & 96 & KDOV OKBK & 8.81202 & 0 & 0 & 9.75 & 0 & 9.75 \\
\hline 14 & 171 & 99 & KDOV OKBK & 8.81202 & 0 & 0 & 4.5 & 0 & 4.5 \\
\hline 14 & 171 & 106 & KDOV OKBK & 8.81202 & 0 & 0 & 2.2 & 0 & 2.2 \\
\hline 14 & 169 & 110 & KGRK $\sim \mathrm{OKBK}$ & 11.26696 & 0 & 0 & 2.9 & 0 & 2.9 \\
\hline 14 & 169 & 71 & $\mathrm{KGRK} \sim \mathrm{OKBK}$ & 11.26696 & 0 & 0 & 9.3 & 0 & 9.3 \\
\hline 14 & 169 & 79 & KGRK $\sim \mathrm{OKBK}$ & 11.26696 & 0 & 0 & 3.6 & 0 & 3.6 \\
\hline 14 & 172 & 125 & KDOV OKAJ & 13.7021 & 0 & 0 & 18.5 & 0 & 18.5 \\
\hline 14 & 172 & 97 & KDOV $\sim \mathrm{OKAJ}$ & 13.7021 & 0 & 0 & 1.5 & 0 & 1.5 \\
\hline 15 & 180 & 14 & KBIF $\sim \mathrm{OKBK}$ & 2.2079 & 0 & 0 & 22.5 & 0 & 22.5 \\
\hline 15 & 181 & 26 & KDOV OKBK & 4.6448 & 0 & 0 & 87.4 & 0 & 87.4 \\
\hline 15 & 182 & 52 & KAEX OKBK & 7.1011 & 0 & 0 & 14.9 & 0 & 14.9 \\
\hline 15 & 183 & 83 & KDOV OKBK & 9.53238 & 0 & 0 & 59.9 & 0 & 59.9 \\
\hline 15 & 183 & 94 & KDOV OKBK & 9.53238 & 0 & 0 & 22.7 & 0 & 22.7 \\
\hline 15 & 183 & 96 & KDOV OKBK & 9.53238 & 0 & 0 & 13.25 & 0 & 13.25 \\
\hline 15 & 184 & 119 & KDOV OEKJ & 11.96293 & 0 & 0 & 23 & 0 & 23 \\
\hline 16 & 192 & 13 & KAGS OKBK & 2.17256 & 0 & 0 & 55 & 0 & 55 \\
\hline 16 & 193 & 41 & KMUO OOTH & 4.63236 & 0 & 0 & 11.1 & 0 & 11.1 \\
\hline 17 & 205 & 34 & KGRK $\sim \mathrm{OKBK}$ & 3.91328 & 0 & 0 & 47.7 & 0 & 47.7 \\
\hline 17 & 204 & 35 & KWRI OKBK & 6.34269 & 0 & 0 & 87.4 & 0 & 87.4 \\
\hline 17 & 206 & 44 & KWRB OEKJ & 8.79009 & 0 & 0 & 60.2 & 0 & 60.2 \\
\hline 17 & 207 & 93 & KDOV OTBD & 11.23951 & 0 & 0 & 3.6 & 0 & 3.6 \\
\hline 17 & 207 & 66 & KDOV OTBD & 11.23951 & 0 & 0 & 3.6 & 0 & 3.6 \\
\hline 17 & 208 & 121 & KDOV OEDR & 13.69153 & 0 & 0 & 4.4 & 0 & 4.4 \\
\hline 18 & 220 & 116 & KTIK OKBK & 13.24948 & 0 & 0 & 35.9 & 0 & 35.9 \\
\hline 19 & 228 & 20 & KDOV OBBS & 2.10262 & 0 & 0 & 18.9 & 0 & 18.9 \\
\hline 19 & 229 & 35 & KWRI OKBK & 4.54281 & 0 & 0 & 87.4 & 0 & 87.4 \\
\hline 19 & 230 & 43 & KDMA $\sim \mathrm{OEKJ}$ & 6.96994 & 0 & 0 & 37.38 & 0 & 37.38 \\
\hline 19 & 232 & 117 & KSUU OKBK & 11.81884 & 0 & 0 & 25.9 & 0 & 25.9 \\
\hline 20 & 240 & 10 & KGRK $\sim \mathrm{OKBK}$ & 2.05202 & 0 & 0 & 0 & 335 & 67 \\
\hline 20 & 240 & 12 & KGRK $\sim \mathrm{OKBK}$ & 2.05202 & 0 & 0 & 0 & 24 & 4.8 \\
\hline
\end{tabular}




\begin{tabular}{|c|c|c|c|c|c|c|c|c|c|}
\hline & & & & & \multicolumn{5}{|c|}{-------DELIVERED QUANTITIES--------- } \\
\hline Tail\# & Msn\# & REQT\# & APOE $\sim$ APOD & ARR APOD & OUT & OVER & BULK & PAX & TOTAL \\
\hline 20 & 242 & 77 & KAGS OKBK & 6.8162 & 0 & 0 & 0 & 30 & 6 \\
\hline 21 & 252 & 10 & KGRK OKBK & 1.88847 & 0 & 0 & 0 & 322 & 64.4 \\
\hline 21 & 252 & 12 & KGRK $\sim \mathrm{OKBK}$ & 1.88847 & 0 & 0 & 0 & 9 & 1.8 \\
\hline 21 & 253 & 46 & KHMN $\sim \mathrm{OKBK}$ & 4.91441 & 0 & 0 & 0 & 312 & 62.4 \\
\hline 22 & 264 & 21 & KGRK OKBK & 1.87178 & 0 & 0 & 0 & 335 & 67 \\
\hline 22 & 265 & 48 & KMUO OTBD & 4.93636 & 0 & 0 & 0 & 335 & 67 \\
\hline 22 & 266 & 83 & KDOV OKBK & 7.77573 & 0 & 0 & 0 & 211 & 42.2 \\
\hline 23 & 276 & 21 & KGRK $\sim \mathrm{OKBK}$ & 1.94263 & 0 & 0 & 0 & 335 & 67 \\
\hline 23 & 277 & 48 & KMUO OTBD & 4.93642 & 0 & 0 & 0 & 96 & 19.2 \\
\hline 23 & 278 & 75 & KDOV OEKJ & 6.83167 & 0 & 0 & 0 & 4 & 0.8 \\
\hline 24 & 288 & 21 & KGRK OKBK & 1.87689 & 0 & 0 & 0 & 49 & 9.8 \\
\hline 24 & 288 & 27 & KGRK $\sim \mathrm{OKBK}$ & 1.87689 & 0 & 0 & 0 & 286 & 57.2 \\
\hline 24 & 289 & 53 & KGRK $\sim \mathrm{OKBK}$ & 4.877 & 0 & 0 & 0 & 273 & 54.6 \\
\hline 24 & 290 & 81 & PHIK OKBK & 7.63356 & 0 & 0 & 0 & 16 & 3.2 \\
\hline 25 & 300 & 27 & KGRK $\sim \mathrm{OKBK}$ & 1.87702 & 0 & 0 & 0 & 335 & 67 \\
\hline 26 & 312 & 27 & KGRK $\sim \mathrm{OKBK}$ & 3.88846 & 0 & 0 & 0 & 119 & 23.8 \\
\hline 26 & 312 & 34 & KGRK $\sim \mathrm{OKBK}$ & 3.88846 & 0 & 0 & 0 & 56 & 11.2 \\
\hline 26 & 313 & 55 & KBIF OOTBD & 6.04799 & 0 & 0 & 0 & 75 & 15 \\
\hline 27 & 324 & 14 & KBIF $\sim \mathrm{OKBK}$ & 1.97641 & 0 & 0 & 0 & 170 & 34 \\
\hline 27 & 325 & 45 & KPOB OKBK & 5.80078 & 0 & 0 & 0 & 39 & 7.8 \\
\hline 27 & 325 & 58 & KРOB OKBK & 5.80078 & 0 & 0 & 0 & 18 & 3.6 \\
\hline 27 & 325 & 59 & KPOB OKBK & 5.80078 & 0 & 0 & 0 & 30 & 6 \\
\hline 28 & 338 & 85 & KDMA OKBK & 7.9177 & 0 & 0 & 0 & 220 & 44 \\
\hline 29 & 348 & 9 & KDOV OKBK & 1.82887 & 0 & 0 & 0 & 17 & 3.4 \\
\hline 29 & 348 & 28 & KDOV OKBK & 1.82887 & 0 & 0 & 0 & 288 & 57.6 \\
\hline 29 & 349 & 44 & KWRB OEKJ & 6.82904 & 0 & 0 & 0 & 209 & 41.8 \\
\hline 29 & 349 & 74 & KWRB OEKJ & 6.82904 & 0 & 0 & 0 & 27 & 5.4 \\
\hline 29 & 350 & 70 & $\mathrm{KDOV} \sim \mathrm{OKBK}$ & 8.7064 & 0 & 0 & 0 & 70 & 14 \\
\hline 29 & 350 & 73 & KDOV OKBK & 8.7064 & 0 & 0 & 0 & 59 & 11.8 \\
\hline 29 & 350 & 78 & KDOV OKBK & 8.7064 & 0 & 0 & 0 & 7 & 1.4 \\
\hline 30 & 361 & 51 & KLFI OKBK & 4.79773 & 0 & 0 & 0 & 148 & 29.6 \\
\hline 30 & 362 & 102 & KPOB $\sim \mathrm{OBBS}$ & 8.80658 & 0 & 0 & 0 & 27 & 5.4 \\
\hline 31 & 373 & 47 & ETAD OKBK & 4.3351 & 0 & 0 & 0 & 2 & 0.4 \\
\hline 31 & 374 & 101 & KAEX OKBK & 8.85741 & 0 & 0 & 0 & 33 & 6.6 \\
\hline 31 & 374 & 52 & KAEX OKBK & 8.85741 & 0 & 0 & 0 & 8 & 1.6 \\
\hline 32 & 384 & 25 & KHOP OEKJ & 2.96754 & 0 & 0 & 0 & 144 & 28.8 \\
\hline 32 & 385 & 49 & KDOV OKBK & 4.83965 & 0 & 0 & 0 & 26 & 5.2 \\
\hline 32 & 385 & 50 & KDOV $\sim \mathrm{OKBK}$ & 4.83965 & 0 & 0 & 0 & 64 & 12.8 \\
\hline 32 & 386 & 100 & KAEX OBBS & 8.87555 & 0 & 0 & 0 & 33 & 6.6 \\
\hline 32 & 386 & 84 & KAEX $\sim \mathrm{OBBS}$ & 8.87555 & 0 & 0 & 0 & 57 & 11.4 \\
\hline 33 & 396 & 24 & KBIF $\sim \mathrm{OEKJ}$ & 3.04517 & 0 & 0 & 0 & 143 & 28.6 \\
\hline 33 & 397 & 43 & KDMA OEKJ & 5.22174 & 0 & 0 & 0 & 160 & 32 \\
\hline 33 & 398 & 112 & KDOV OKBK & 10.78298 & 0 & 0 & 0 & 88 & 17.6 \\
\hline 33 & 398 & 130 & KDOV OKBK & 10.78298 & 0 & 0 & 0 & 4 & 0.8 \\
\hline 33 & 398 & 99 & KDOV OKBK & 10.78298 & 0 & 0 & 0 & 35 & 7 \\
\hline 33 & 398 & 106 & KDOV OKBK & 10.78298 & 0 & 0 & 0 & 21 & 4.2 \\
\hline 34 & 408 & 57 & KMGE $\sim \mathrm{OKBK}$ & 5.82185 & 0 & 0 & 0 & 98 & 19.6 \\
\hline 34 & 409 & 62 & KBIF OKBK & 7.96511 & 0 & 0 & 0 & 127 & 25.4 \\
\hline 34 & 409 & 82 & KBIF $\sim \mathrm{OKBK}$ & 7.96511 & 0 & 0 & 0 & 40 & 8 \\
\hline 35 & 420 & 1 & KBIF OKBK & 1.24288 & 0 & 0 & 56.2 & 0 & 56.2 \\
\hline 35 & 422 & 35 & KWRI OKBK & 3.81144 & 0 & 0 & 87.4 & 0 & 87.4 \\
\hline 35 & 423 & 82 & KBIF $\sim \mathrm{OKBK}$ & 7.94815 & 0 & 0 & 58.8 & 0 & 58.8 \\
\hline 35 & 421 & 108 & KCHS OBBI & 12.28922 & 0 & 0 & 22.8 & 0 & 22.8 \\
\hline 35 & 425 & 115 & KNGU OKBK & 14.29997 & 0 & 0 & 16.9 & 0 & 16.9 \\
\hline 35 & 425 & 88 & KNGU OKBK & 14.29997 & 0 & 0 & 11.4 & 0 & 11.4 \\
\hline 36 & 432 & 5 & KWRI OKBK & 0.96239 & 0 & 0 & 87.4 & 0 & 87.4 \\
\hline 36 & 437 & 16 & KCHS OBBI & 3.02069 & 0 & 0 & 9.9 & 0 & 9.9 \\
\hline 36 & 433 & 17 & KNGP OBBI & 5.2416 & 0 & 0 & 57 & 0 & 57 \\
\hline
\end{tabular}




\begin{tabular}{|c|c|c|c|c|c|c|c|c|c|}
\hline & & & & & \multicolumn{5}{|c|}{-------DELIVERED QUANTITIES-------- } \\
\hline Tail\# & Msn\# & REQT\# & APOE APOD & ARR APOD & OUT & OVER & BULK & PAX & TOTAL \\
\hline 36 & 434 & 35 & KWRI OKBK & 7.22651 & 0 & 0 & 87.4 & 0 & 87.4 \\
\hline 36 & 436 & 89 & KTIK OKBK & 9.37787 & 0 & 0 & 23.2 & 0 & 23.2 \\
\hline 37 & 445 & 19 & $\mathrm{KSKF} \sim \mathrm{OKBK}$ & 3.17781 & 0 & 0 & 22.3 & 0 & 22.3 \\
\hline 37 & 446 & 35 & KWRI OKBK & 5.13921 & 0 & 0 & 87.4 & 0 & 87.4 \\
\hline 37 & 448 & 90 & KSUU OKBK & 9.48716 & 0 & 0 & 17.4 & 0 & 17.4 \\
\hline 37 & 449 & 118 & $\mathrm{KSKF} \sim \mathrm{OKBK}$ & 11.686 & 0 & 0 & 28.8 & 0 & 28.8 \\
\hline 38 & 456 & 3 & KDOV OKBK & 1.82868 & 0 & 0 & 4.8 & 0 & 4.8 \\
\hline 38 & 456 & 9 & KDOV OKBK & 1.82868 & 0 & 0 & 3.4 & 0 & 3.4 \\
\hline 38 & 457 & 25 & KHOP $\sim \mathrm{OEKJ}$ & 3.91994 & 0 & 0 & 87.4 & 0 & 87.4 \\
\hline 38 & 458 & 35 & KWRI OKBK & 5.89223 & 0 & 0 & 87.4 & 0 & 87.4 \\
\hline 38 & 459 & 59 & KРOB $\sim \mathrm{OKBK}$ & 7.92781 & 0 & 0 & 1.4 & 0 & 1.4 \\
\hline 38 & 461 & 131 & $\mathrm{KGRK} \sim \mathrm{OKBK}$ & 12.11096 & 0 & 0 & 32.8 & 0 & 32.8 \\
\hline 39 & 468 & 7 & KDOV OBBS & 0.92115 & 0 & 0 & 43.8 & 0 & 43.8 \\
\hline 39 & 469 & 25 & KHOP $\sim \mathrm{OEKJ}$ & 3.14519 & 0 & 0 & 87.4 & 0 & 87.4 \\
\hline 39 & 470 & 35 & KWRI OKBK & 5.12172 & 0 & 0 & 87.4 & 0 & 87.4 \\
\hline 39 & 471 & 87 & KWRI OKBK & 7.80635 & 0 & 0 & 69.7 & 0 & 69.7 \\
\hline 39 & 471 & 95 & KWRI OKBK & 7.80635 & 0 & 0 & 11.1 & 0 & 11.1 \\
\hline 39 & 472 & 92 & $\mathrm{KSKF} \sim \mathrm{OKBK}$ & 10.00299 & 0 & 0 & 25.4 & 0 & 25.4 \\
\hline 39 & 472 & 64 & $\mathrm{KSKF} \sim \mathrm{OKBK}$ & 10.00299 & 0 & 0 & 22.3 & 0 & 22.3 \\
\hline 39 & 473 & 128 & $\mathrm{KMGE} \sim \mathrm{OKBK}$ & 12.06817 & 0 & 0 & 21 & 0 & 21 \\
\hline 40 & 481 & 104 & KGRK OKBK & 11.01687 & 0 & 0 & 27.6 & 0 & 27.6 \\
\hline 40 & 481 & 127 & KGRK $\sim \mathrm{OKBK}$ & 11.01687 & 0 & 0 & 16.9 & 0 & 16.9 \\
\hline 40 & 481 & 53 & $\mathrm{KGRK} \sim \mathrm{OKBK}$ & 11.01687 & 0 & 0 & 12 & 0 & 12 \\
\hline 41 & 493 & 33 & KBIF $\sim \mathrm{OBBS}$ & 4.18807 & 0 & 0 & 79.2 & 0 & 79.2 \\
\hline 41 & 492 & 61 & KDOV OKBK & 6.16684 & 0 & 0 & 36.6 & 0 & 36.6 \\
\hline 41 & 492 & 65 & KDOV OKBK & 6.16684 & 0 & 0 & 2.7 & 0 & 2.7 \\
\hline 41 & 492 & 67 & KDOV OKBK & 6.16684 & 0 & 0 & 50.58 & 0 & 50.58 \\
\hline 42 & 504 & 67 & $\mathrm{KDOV} \sim \mathrm{OKBK}$ & 5.81716 & 0 & 0 & 24.42 & 0 & 24.42 \\
\hline 42 & 504 & 70 & KDOV OKBK & 5.81716 & 0 & 0 & 58.28 & 0 & 58.28 \\
\hline 42 & 505 & 84 & KAEX $\sim \mathrm{OBBS}$ & 7.97584 & 0 & 0 & 3.3 & 0 & 3.3 \\
\hline 43 & 517 & 18 & KWRI OKBK & 3.90389 & 0 & 0 & 48.2 & 0 & 48.2 \\
\hline 43 & 516 & 39 & KDOV OBBS & 5.88775 & 0 & 0 & 17.9 & 0 & 17.9 \\
\hline 44 & 529 & 80 & KRIV OKBK & 8.1667 & 0 & 0 & 64.2 & 0 & 64.2 \\
\hline 45 & 540 & 68 & KDOV OKAJ & 5.81602 & 0 & 0 & 21.5 & 0 & 21.5 \\
\hline 45 & 541 & 114 & KRIV OKBK & 9.99202 & 0 & 0 & 74 & 0 & 74 \\
\hline 46 & 552 & 37 & $\mathrm{KSKF} \sim \mathrm{OKBK}$ & 4.13362 & 0 & 0 & 22.3 & 0 & 22.3 \\
\hline 46 & 553 & 113 & KWRI OKBK & 9.80635 & 0 & 0 & 11.11 & 0 & 11.11 \\
\hline 46 & 553 & 123 & KWRI OKBK & 9.80635 & 0 & 0 & 78.77 & 0 & 78.77 \\
\hline 47 & 565 & 123 & KWRI OKBK & 9.86977 & 0 & 0 & 82.7 & 0 & 82.7 \\
\hline 48 & 577 & 123 & KWRI OKBK & 9.92821 & 0 & 0 & 82.7 & 0 & 82.7 \\
\hline 49 & 588 & 57 & KMGE OKBK & 6.87478 & 0 & 0 & 19.5 & 0 & 19.5 \\
\hline 49 & 588 & 72 & KMGE $\sim \mathrm{OKBK}$ & 6.87478 & 0 & 0 & 5.6 & 0 & 5.6 \\
\hline 49 & 589 & 123 & KWRI OKBK & 9.88138 & 0 & 0 & 82.7 & 0 & 82.7 \\
\hline 50 & 601 & 123 & KWRI OKBK & 9.80636 & 0 & 0 & 82.7 & 0 & 82.7 \\
\hline 51 & 613 & 123 & KWRI OKBK & 9.87715 & 0 & 0 & 82.7 & 0 & 82.7 \\
\hline 52 & 624 & 44 & KWRB OEKJ & 4.86313 & 0 & 0 & 72.2 & 0 & 72.2 \\
\hline 52 & 625 & 123 & KWRI OKBK & 9.86977 & 0 & 0 & 82.7 & 0 & 82.7 \\
\hline 53 & 637 & 123 & KWRI OKBK & 9.86982 & 0 & 0 & 36.73 & 0 & 36.73 \\
\hline 55 & 660 & 2 & $\mathrm{KGRK} \sim \mathrm{OKBK}$ & 1.43354 & 34.5 & 2 & 0 & 0 & 36.5 \\
\hline 55 & 661 & 7 & KDOV OBBS & 3.66387 & 29 & 0 & 0 & 0 & 29 \\
\hline 55 & 663 & 48 & KMUO $\sim \mathrm{OTBD}$ & 6.60399 & 0 & 39.22 & 0 & 0 & 39.22 \\
\hline 55 & 664 & 80 & KRIV OKBK & 9.73223 & 0 & 62.4 & 0 & 0 & 62.4 \\
\hline 55 & 665 & 85 & KDMA $\sim \mathrm{OKBK}$ & 12.63489 & 0 & 55.91 & 0 & 0 & 55.91 \\
\hline 55 & 666 & 101 & KAEX $\sim \mathrm{OKBK}$ & 15.38405 & 31.5 & 36.5 & 0 & 0 & 68 \\
\hline 55 & 662 & 111 & KHOP OKBK & 18.06055 & 0 & 63.79 & 5.69 & 51 & 79.68 \\
\hline 56 & 672 & 2 & KGRK $\sim \mathrm{OKBK}$ & 1.4431 & 34.5 & 0 & 0 & 0 & 34.5 \\
\hline 56 & 673 & 7 & KDOV OBBS & 4.16457 & 29 & 0 & 0 & 0 & 29 \\
\hline 56 & 674 & 33 & KBIF $\sim \mathrm{OBBS}$ & 7.19957 & 0 & 51.2 & 0 & 0 & 51.2 \\
\hline
\end{tabular}




\begin{tabular}{|c|c|c|c|c|c|c|c|c|c|}
\hline & & & & & \multicolumn{5}{|c|}{-------DELIVERED QUANTITIES-------- } \\
\hline Tail\# & Msn\# & REQT\# & APOE APOD & ARR APOD & OUT & OVER & BULK & PAX & TOTAL \\
\hline 56 & 674 & 60 & $\mathrm{KBIF} \sim \mathrm{OBBS}$ & 7.19957 & 0 & 0 & 4.2 & 0 & 4.2 \\
\hline 56 & 675 & 48 & KMUO OTBD & 10.3012 & 0 & 64.6 & 0 & 0 & 64.6 \\
\hline 56 & 676 & 80 & KRIV OKBK & 13.26815 & 0.6 & 62.16 & 0 & 71 & 76.96 \\
\hline 56 & 678 & 84 & KAEX $\sim \mathrm{OBBS}$ & 16.03399 & 2.1 & 28.1 & 0 & 0 & 30.2 \\
\hline 56 & 677 & 89 & KTIK $\sim \mathrm{OKBK}$ & 18.81674 & 3 & 0 & 0 & 0 & 3 \\
\hline 57 & 684 & 2 & KGRK $\sim \mathrm{OKBK}$ & 2.16848 & 30.8 & 0 & 0 & 0 & 30.8 \\
\hline 57 & 684 & 21 & KGRK $\sim \mathrm{OKBK}$ & 2.16848 & 0 & 0 & 11.6 & 0 & 11.6 \\
\hline 57 & 684 & 27 & KGRK $\sim \mathrm{OKBK}$ & 2.16848 & 0 & 0 & 0.4 & 0 & 0.4 \\
\hline 57 & 686 & 54 & KBIF $\sim \mathrm{OBBS}$ & 6.4588 & 34.5 & 16.9 & 0 & 0 & 51.4 \\
\hline 57 & 687 & 54 & KBIF $\sim \mathrm{OBBS}$ & 9.34664 & 34.5 & 5.9 & 0 & 0 & 40.4 \\
\hline 57 & 688 & 80 & KRIV OKBK & 12.45978 & 0 & 62.4 & 0 & 71 & 76.6 \\
\hline 57 & 689 & 90 & KSUU OKBK & 15.44378 & 20 & 0 & 0 & 0 & 20 \\
\hline 57 & 690 & 100 & KAEX $\sim \mathrm{OBBS}$ & 18.35698 & 31.5 & 39.2 & 0 & 0 & 70.7 \\
\hline 58 & 696 & 1 & KBIF $\sim \mathrm{OKBK}$ & 1.51554 & 34.5 & 16.9 & 0 & 0 & 51.4 \\
\hline 58 & 697 & 4 & KDMA OKBK & 4.41366 & 0 & 47.6 & 0 & 12 & 50 \\
\hline 58 & 698 & 54 & KBIF $\sim \mathrm{OBBS}$ & 7.29428 & 34.5 & 16.9 & 0 & 0 & 51.4 \\
\hline 58 & 699 & 84 & KAEX $\sim \mathrm{OBBS}$ & 10.06809 & 34.5 & 16.9 & 0 & 0 & 51.4 \\
\hline 58 & 701 & 92 & $\mathrm{KSKF} \sim \mathrm{OKBK}$ & 12.88776 & 0 & 6 & 0 & 0 & 6 \\
\hline 58 & 702 & 130 & KDOV OKBK & 15.16028 & 0 & 0 & 60.4 & 0 & 60.4 \\
\hline 59 & 708 & 1 & $\mathrm{KBIF} \sim \mathrm{OKBK}$ & 1.50585 & 34.5 & 16.9 & 0 & 0 & 51.4 \\
\hline 59 & 709 & 8 & KNGP OBBI & 4.77514 & 29 & 13.9 & 0 & 0 & 42.9 \\
\hline 59 & 710 & 31 & KPOB OEKJ & 7.40807 & 0 & 18.9 & 0 & 0 & 18.9 \\
\hline 59 & 710 & 76 & KPOB OEKJ & 7.40807 & 0 & 0 & 10.2 & 0 & 10.2 \\
\hline 59 & 711 & 54 & KBIF $\sim \mathrm{OBBS}$ & 10.29079 & 34.5 & 0 & 0 & 0 & 34.5 \\
\hline 59 & 712 & 104 & KGRK $\sim \mathrm{OKBK}$ & 13.10362 & 34.5 & 16.9 & 0 & 0 & 51.4 \\
\hline 59 & 714 & 105 & KDOV OBBS & 15.52342 & 0.7 & 1.3 & 0 & 0 & 2 \\
\hline 59 & 714 & 7 & KDOV $\sim \mathrm{OBBS}$ & 15.52342 & 7 & 0 & 0 & 0 & 7 \\
\hline 59 & 713 & 131 & $\mathrm{KGRK} \sim \mathrm{OKBK}$ & 18.33173 & 34.5 & 16.9 & 0 & 0 & 51.4 \\
\hline 60 & 720 & 1 & KBIF $\sim \mathrm{OKBK}$ & 1.51554 & 34.5 & 16.9 & 0 & 0 & 51.4 \\
\hline 60 & 721 & 8 & KNGP OBBI & 7.05223 & 24 & 43.47 & 0 & 0 & 67.47 \\
\hline 60 & 723 & 54 & KBIF OBBS & 12.06589 & 34.5 & 0 & 0 & 0 & 34.5 \\
\hline 60 & 724 & 80 & KRIV OKBK & 15.17839 & 0 & 1.54 & 0 & 0 & 1.54 \\
\hline 60 & 726 & 107 & KDOV $\sim \mathrm{OBBI}$ & 17.31153 & 7.9 & 0 & 0 & 6 & 9.1 \\
\hline 61 & 732 & 1 & KBIF $\sim \mathrm{OKBK}$ & 1.50585 & 34.5 & 16.9 & 0 & 0 & 51.4 \\
\hline 61 & 733 & 17 & KNGP OBBI & 4.32214 & 29 & 13.9 & 0 & 0 & 42.9 \\
\hline 61 & 735 & 60 & KBIF $\sim \mathrm{OBBS}$ & 7.21982 & 34.5 & 16.9 & 0 & 0 & 51.4 \\
\hline 61 & 736 & 72 & KMGE OKBK & 9.94717 & 12 & 25.1 & 0 & 0 & 37.1 \\
\hline 61 & 738 & 103 & KSVN OBBS & 12.62031 & 12.6 & 12 & 0 & 17 & 28 \\
\hline 61 & 734 & 113 & KWRI OKBK & 15.20147 & 0 & 0 & 70.99 & 7 & 72.39 \\
\hline 61 & 737 & 131 & KGRK $\sim \mathrm{OKBK}$ & 18.00184 & 34.5 & 16.9 & 0 & 0 & 51.4 \\
\hline 62 & 744 & 1 & KBIF O OKBK & 1.56418 & 34.2 & 0 & 0 & 0 & 34.2 \\
\hline 62 & 745 & 8 & KNGP $\sim \mathrm{OBBI}$ & 4.38512 & 0 & 62.4 & 0 & 0 & 62.4 \\
\hline 62 & 747 & 54 & KBIF $\sim \mathrm{OBBS}$ & 7.27314 & 50.7 & 0 & 0 & 0 & 50.7 \\
\hline 62 & 746 & 63 & KWRI OKBK & 9.85935 & 0 & 0 & 53 & 0 & 53 \\
\hline 62 & 748 & 76 & KPOB OEKJ & 12.4702 & 37 & 11.2 & 0 & 0 & 48.2 \\
\hline 62 & 749 & 110 & KGRK $\sim \mathrm{OKBK}$ & 15.25424 & 0 & 63.79 & 0 & 0 & 63.79 \\
\hline 62 & 750 & 111 & KHOP $\sim \mathrm{OKBK}$ & 18.09182 & 0 & 6.96 & 0 & 0 & 6.96 \\
\hline 63 & 756 & 1 & KBIF $\sim \mathrm{OKBK}$ & 1.61266 & 34.5 & 4.1 & 0 & 0 & 38.6 \\
\hline 63 & 757 & 8 & KNGP $\sim \mathrm{OBBI}$ & 4.68762 & 0 & 62.4 & 0 & 0 & 62.4 \\
\hline 63 & 758 & 35 & KWRI OKBK & 7.42578 & 0 & 0 & 70.99 & 0 & 70.99 \\
\hline 63 & 761 & 131 & KGRK $\sim \mathrm{OKBK}$ & 15.7677 & 34.5 & 16.9 & 0 & 0 & 51.4 \\
\hline 64 & 768 & 0 & KMGE $\sim \mathrm{OKBK}$ & 1.36234 & 0 & 59.23 & 0 & 55 & 70.23 \\
\hline 64 & 770 & 30 & ETAD OOTH & 3.81297 & 0 & 0 & 51.7 & 3 & 52.3 \\
\hline 64 & 771 & 55 & KBIF $\sim \mathrm{OTBD}$ & 6.83022 & 0 & 40.35 & 13.2 & 0 & 53.55 \\
\hline 64 & 772 & 76 & KPOB OEKJ & 9.46868 & 34.5 & 16.9 & 0 & 0 & 51.4 \\
\hline 64 & 769 & 84 & KAEX $\sim \mathrm{OBBS}$ & 12.2226 & 34.5 & 16.9 & 0 & 0 & 51.4 \\
\hline 64 & 773 & 110 & KGRK $\sim \mathrm{OKBK}$ & 15.02108 & 0 & 12.61 & 0 & 0 & 12.61 \\
\hline 64 & 773 & 131 & KGRK $\sim \mathrm{OKBK}$ & 15.02108 & 0 & 14.89 & 0 & 0 & 14.89 \\
\hline
\end{tabular}




\begin{tabular}{|c|c|c|c|c|c|c|c|c|c|}
\hline & & & & & \multicolumn{5}{|c|}{--------DELIVERED QUANTITIES--------- } \\
\hline Tail\# & Msn\# & REQT\# & APOE $\sim$ APOD & ARR APOD & OUT & OVER & BULK & PAX & TOTAL \\
\hline 65 & 780 & 26 & KDOV OKBK & 5.90345 & 0 & 0 & 54.65 & 0 & 54.65 \\
\hline 65 & 780 & 70 & KDOV OKBK & 5.90345 & 0 & 0 & 9.02 & 0 & 9.02 \\
\hline 65 & 781 & 111 & KHOP OKBK & 10.17684 & 0 & 63.79 & 0 & 0 & 63.79 \\
\hline 66 & 792 & 71 & KGRK OKBK & 7.16862 & 0 & 59.23 & 0 & 0 & 59.23 \\
\hline 66 & 792 & 79 & KGRK $\sim \mathrm{OKBK}$ & 7.16862 & 2.97 & 13.11 & 0 & 0 & 16.07 \\
\hline 66 & 793 & 111 & KHOP $\sim \mathrm{OKBK}$ & 10.16716 & 0 & 59.23 & 0 & 0 & 59.23 \\
\hline 67 & 804 & 71 & KGRK $\sim \mathrm{OKBK}$ & 7.17819 & 0 & 56.86 & 0 & 0 & 56.86 \\
\hline 67 & 804 & 79 & KGRK $\sim \mathrm{OKBK}$ & 7.17819 & 0 & 2.22 & 0 & 0 & 2.22 \\
\hline 67 & 805 & 109 & KGRK $\sim \mathrm{OKBK}$ & 9.97418 & 29.4 & 38.3 & 0 & 0 & 67.7 \\
\hline 68 & 816 & 104 & KGRK $\sim \mathrm{OKBK}$ & 9.17823 & 34.5 & 16.9 & 0 & 71 & 65.6 \\
\hline 68 & 817 & 127 & KGRK OKBK & 12.11617 & 34.5 & 16.9 & 0 & 0 & 51.4 \\
\hline 69 & 829 & 99 & KDOV OKBK & 8.91305 & 0.3 & 45 & 0 & 0 & 45.3 \\
\hline 69 & 828 & 104 & KGRK $\sim \mathrm{OKBK}$ & 11.70392 & 34.5 & 16.9 & 0 & 71 & 65.6 \\
\hline 70 & 840 & 104 & KGRK $\sim \mathrm{OKBK}$ & 9.17823 & 34.5 & 16.9 & 0 & 71 & 65.6 \\
\hline 70 & 841 & 122 & KDOV OTBD & 11.31709 & 0 & 33 & 31.6 & 0 & 64.6 \\
\hline 71 & 852 & 104 & KGRK $\sim \mathrm{OKBK}$ & 10.84825 & 34.5 & 16.9 & 0 & 46 & 60.6 \\
\hline 71 & 852 & 109 & KGRK OKBK & 10.84825 & 0 & 0 & 0 & 15 & 3 \\
\hline 71 & 852 & 127 & KGRK $\sim \mathrm{OKBK}$ & 10.84825 & 0 & 0 & 0 & 10 & 2 \\
\hline 72 & 864 & 104 & KGRK OKBK & 9.16847 & 34.5 & 16.9 & 0 & 0 & 51.4 \\
\hline 72 & 864 & 127 & KGRK $\sim \mathrm{OKBK}$ & 9.16847 & 0 & 0 & 0 & 5 & 1 \\
\hline 72 & 865 & 119 & KDOV OEKJ & 11.30118 & 0 & 3.21 & 63.21 & 0 & 66.42 \\
\hline 73 & 876 & 104 & KGRK $\sim \mathrm{OKBK}$ & 9.16846 & 34.5 & 16.9 & 0 & 0 & 51.4 \\
\hline 73 & 877 & 119 & KDOV OEKJ & 11.28692 & 0 & 63.79 & 5.69 & 0 & 69.48 \\
\hline 74 & 888 & 0 & KMGE $\sim \mathrm{OKBK}$ & 1.21665 & 26.2 & 11.5 & 0 & 28 & 43.3 \\
\hline 74 & 889 & 12 & KGRK $\sim \mathrm{OKBK}$ & 3.70666 & 0 & 9.56 & 0 & 0 & 9.56 \\
\hline 74 & 889 & 21 & KGRK $\sim \mathrm{OKBK}$ & 3.70666 & 26.2 & 11.5 & 0 & 0 & 37.7 \\
\hline 74 & 891 & 55 & KBIF $\sim \mathrm{OTBD}$ & 6.41079 & 26.2 & 11.5 & 0 & 0 & 37.7 \\
\hline 74 & 890 & 71 & $\mathrm{KGRK} \sim \mathrm{OKBK}$ & 8.91879 & 8 & 51.41 & 0 & 0 & 59.41 \\
\hline 74 & 890 & 79 & KGRK OKBK & 8.91879 & 12.43 & 9.77 & 0 & 0 & 22.21 \\
\hline 74 & 892 & 77 & KAGS $\sim \mathrm{OKBK}$ & 11.63572 & 0 & 14.1 & 2 & 0 & 16.1 \\
\hline 74 & 893 & 127 & KGRK $\sim \mathrm{OKBK}$ & 14.2202 & 0 & 42.19 & 0 & 0 & 42.19 \\
\hline 74 & 894 & 117 & KSUU OKBK & 16.93428 & 12.6 & 0 & 0 & 0 & 12.6 \\
\hline 75 & 900 & 0 & $\mathrm{KMGE} \sim \mathrm{OKBK}$ & 1.13654 & 11.8 & 30.89 & 0 & 27 & 48.09 \\
\hline 75 & 901 & 10 & KGRK $\sim \mathrm{OKBK}$ & 3.65977 & 16.6 & 0 & 0 & 0 & 16.6 \\
\hline 75 & 901 & 11 & KGRK $\sim \mathrm{OKBK}$ & 3.65977 & 0 & 16.84 & 0 & 0 & 16.84 \\
\hline 75 & 901 & 12 & KGRK $\sim \mathrm{OKBK}$ & 3.65977 & 0.6 & 15.84 & 0 & 0 & 16.44 \\
\hline 75 & 903 & 55 & KBIF $\sim \mathrm{OTBD}$ & 6.25052 & 26.2 & 11.5 & 0 & 0 & 37.7 \\
\hline 75 & 902 & 69 & KDOV OBBS & 8.77158 & 0 & 0 & 39.2 & 10 & 41.2 \\
\hline 75 & 904 & 74 & KWRB OEKJ & 11.3026 & 26.2 & 11.5 & 0 & 0 & 37.7 \\
\hline 75 & 905 & 127 & KGRK $\sim \mathrm{OKBK}$ & 13.96494 & 0 & 47.9 & 0 & 0 & 47.9 \\
\hline 75 & 906 & 117 & KSUU OKBK & 16.7124 & 26.2 & 0 & 0 & 0 & 26.2 \\
\hline 76 & 912 & 0 & KMGE OKBK & 1.36256 & 0 & 47.9 & 0 & 28 & 53.5 \\
\hline 76 & 913 & 10 & KGRK OKBK & 3.94705 & 26.2 & 11.5 & 0 & 0 & 37.7 \\
\hline 76 & 913 & 11 & KGRK $\sim \mathrm{OKBK}$ & 3.94705 & 6.17 & 4.85 & 0 & 0 & 11.03 \\
\hline 76 & 914 & 32 & KDMA $\sim \mathrm{OBBS}$ & 6.75077 & 0 & 47.9 & 0 & 0 & 47.9 \\
\hline 76 & 915 & 55 & KBIF $\sim \mathrm{OTBD}$ & 9.44843 & 26.2 & 11.5 & 0 & 0 & 37.7 \\
\hline 76 & 916 & 74 & KWRB OEKJ & 11.8437 & 26.2 & 4.7 & 0 & 0 & 30.9 \\
\hline 76 & 917 & 127 & KGRK OKBK & 14.2512 & 26.2 & 11.5 & 0 & 0 & 37.7 \\
\hline 76 & 918 & 117 & KSUU OKBK & 17.13996 & 26.2 & 0 & 0 & 0 & 26.2 \\
\hline 77 & 924 & 0 & KMGE OKBK & 1.42089 & 0 & 47.9 & 0 & 28 & 53.5 \\
\hline 77 & 925 & 10 & KGRK $\sim \mathrm{OKBK}$ & 4.0243 & 26.2 & 0 & 0 & 0 & 26.2 \\
\hline 77 & 925 & 11 & KGRK $\sim \mathrm{OKBK}$ & 4.0243 & 0 & 52.07 & 0 & 0 & 52.07 \\
\hline 77 & 926 & 32 & KDMA OBBS & 6.74784 & 0 & 42.19 & 0 & 0 & 42.19 \\
\hline 77 & 927 & 55 & KBIF $\sim \mathrm{OTBD}$ & 9.35104 & 26.2 & 11.5 & 0 & 0 & 37.7 \\
\hline 77 & 928 & 74 & KWRB OEKJ & 11.74628 & 26.2 & 0 & 0 & 0 & 26.2 \\
\hline 77 & 929 & 127 & KGRK $\sim \mathrm{OKBK}$ & 14.32844 & 0 & 39.61 & 0 & 0 & 39.61 \\
\hline 77 & 930 & 115 & KNGU OKBK & 16.81233 & 26.2 & 0 & 0 & 0 & 26.2 \\
\hline 78 & 936 & 0 & KMGE OKBK & 1.36252 & 0 & 47.9 & 0 & 0 & 47.9 \\
\hline
\end{tabular}




\begin{tabular}{|c|c|c|c|c|c|c|c|c|c|}
\hline & & & & & \multicolumn{5}{|c|}{--------DELIVERED QUANTITIES--------- } \\
\hline Tail\# & Msn\# & REQT\# & APOE $\sim$ APOD & ARR APOD & OUT & OVER & BULK & PAX & TOTAL \\
\hline 78 & 937 & 21 & $\mathrm{KGRK} \sim \mathrm{OKBK}$ & 3.8525 & 0 & 4.47 & 0 & 0 & 4.47 \\
\hline 78 & 937 & 27 & KGRK OKBK & 3.8525 & 0 & 39.1 & 0 & 0 & 39.1 \\
\hline 78 & 938 & 32 & KDMA $\sim \mathrm{OBBS}$ & 6.66061 & 0 & 33.51 & 0 & 0 & 33.51 \\
\hline 78 & 939 & 55 & KBIF $\sim \mathrm{OTBD}$ & 9.35828 & 14.7 & 30.65 & 0 & 0 & 45.35 \\
\hline 78 & 940 & 74 & KWRB OEKJ & 12.47805 & 23.4 & 0 & 0 & 0 & 23.4 \\
\hline 78 & 941 & 110 & KGRK $\sim \mathrm{OKBK}$ & 14.8856 & 17.7 & 28.3 & 0 & 0 & 46 \\
\hline 78 & 942 & 115 & KNGU OKBK & 17.28946 & 17.8 & 0 & 0 & 0 & 17.8 \\
\hline 79 & 948 & 0 & KMGE OKBK & 1.13654 & 0 & 42.19 & 0 & 24 & 46.99 \\
\hline 79 & 949 & 10 & KGRK OKBK & 3.62656 & 26.2 & 11.5 & 0 & 0 & 37.7 \\
\hline 79 & 949 & 11 & KGRK $\sim \mathrm{OKBK}$ & 3.62656 & 6.53 & 35.86 & 0 & 0 & 42.39 \\
\hline 79 & 950 & 32 & KDMA OBBS & 6.40148 & 0 & 47.9 & 0 & 5 & 48.9 \\
\hline 79 & 951 & 55 & KBIF $\sim \mathrm{OTBD}$ & 9.03797 & 26.2 & 11.5 & 0 & 0 & 37.7 \\
\hline 79 & 952 & 74 & KWRB OEKJ & 11.54571 & 26.2 & 0 & 0 & 0 & 26.2 \\
\hline 79 & 953 & 127 & KGRK $\sim \mathrm{OKBK}$ & 14.14676 & 0 & 47.9 & 0 & 0 & 47.9 \\
\hline 80 & 960 & 0 & KMGE OKBK & 1.19482 & 0 & 36.29 & 0 & 0 & 36.29 \\
\hline 80 & 961 & 21 & KGRK $\sim \mathrm{OKBK}$ & 3.71805 & 5.3 & 38.03 & 0 & 0 & 43.33 \\
\hline 80 & 962 & 40 & KTIK OEKJ & 6.34386 & 0 & 0 & 1.06 & 0 & 1.06 \\
\hline 80 & 963 & 45 & KPOB OKBK & 8.77545 & 0 & 33 & 0 & 0 & 33 \\
\hline 80 & 963 & 59 & KРOB OKBK & 8.77545 & 0 & 13.29 & 0 & 0 & 13.29 \\
\hline 80 & 964 & 74 & KWRB OEKJ & 11.50571 & 26.2 & 11.5 & 0 & 0 & 37.7 \\
\hline 80 & 965 & 127 & KGRK OKBK & 14.02656 & 26.2 & 11.5 & 0 & 0 & 37.7 \\
\hline 80 & 966 & 108 & KCHS OBBI & 16.31437 & 10 & 5.5 & 0 & 0 & 15.5 \\
\hline 80 & 966 & 16 & KCHS $\sim \mathrm{OBBI}$ & 16.31437 & 0 & 0 & 0 & 16 & 3.2 \\
\hline 81 & 972 & 5 & KWRI OKBK & 1.32761 & 49.6 & 0 & 0 & 0 & 49.6 \\
\hline 81 & 973 & 10 & KGRK $\sim \mathrm{OKBK}$ & 3.85085 & 26.2 & 1.7 & 0 & 0 & 27.9 \\
\hline 81 & 973 & 11 & KGRK $\sim \mathrm{OKBK}$ & 3.85085 & 0 & 20.37 & 0 & 0 & 20.37 \\
\hline 81 & 974 & 40 & KTIK OEKJ & 6.30596 & 0 & 37.7 & 10.44 & 0 & 48.14 \\
\hline 81 & 975 & 44 & KWRB OEKJ & 8.68091 & 0 & 28.86 & 0 & 0 & 28.86 \\
\hline 81 & 975 & 74 & KWRB OEKJ & 8.68091 & 0 & 0 & 1.7 & 0 & 1.7 \\
\hline 81 & 976 & 73 & KDOV OKBK & 11.07471 & 0 & 21.81 & 0 & 0 & 21.81 \\
\hline 81 & 976 & 49 & KDOV OKBK & 11.07471 & 0 & 0 & 0.3 & 0 & 0.3 \\
\hline 81 & 976 & 50 & KDOV OKBK & 11.07471 & 0 & 5.4 & 4.4 & 0 & 9.8 \\
\hline 81 & 977 & 127 & KGRK $\sim \mathrm{OKBK}$ & 13.59791 & 26.2 & 11.5 & 0 & 0 & 37.7 \\
\hline 81 & 978 & 131 & KGRK $\sim \mathrm{OKBK}$ & 16.00772 & 26.2 & 11.5 & 0 & 0 & 37.7 \\
\hline 82 & 984 & 5 & KWRI OKBK & 1.18924 & 26.2 & 0 & 9 & 0 & 35.2 \\
\hline 82 & 985 & 13 & KAGS OKBK & 3.69461 & 26.2 & 11.5 & 0 & 0 & 37.7 \\
\hline 82 & 986 & 40 & KTIK OEKJ & 6.34941 & 0 & 41.9 & 0 & 0 & 41.9 \\
\hline 82 & 987 & 44 & KWRB $\sim \mathrm{OEKJ}$ & 8.6912 & 0 & 41.9 & 0 & 0 & 41.9 \\
\hline 82 & 988 & 70 & KDOV OKBK & 11.25972 & 0 & 32.7 & 0 & 0 & 32.7 \\
\hline 82 & 989 & 127 & KGRK $\sim \mathrm{OKBK}$ & 13.78295 & 0 & 42.19 & 0 & 0 & 42.19 \\
\hline 82 & 990 & 131 & KGRK $\sim \mathrm{OKBK}$ & 16.44748 & 26.2 & 11.5 & 0 & 0 & 37.7 \\
\hline 83 & 996 & 5 & KWRI OKBK & 1.32761 & 26.2 & 0 & 0 & 0 & 26.2 \\
\hline 83 & 997 & 13 & KAGS OKBK & 3.57827 & 26.2 & 11.5 & 0 & 0 & 37.7 \\
\hline 83 & 998 & 40 & KTIK OEKJ & 6.09169 & 0 & 41.9 & 0 & 0 & 41.9 \\
\hline 83 & 999 & 44 & KWRB OEKJ & 8.52801 & 11.9 & 35.44 & 0 & 0 & 47.34 \\
\hline 83 & 1000 & 75 & KDOV OEKJ & 10.95289 & 0 & 2 & 4 & 0 & 6 \\
\hline 83 & 1000 & 91 & KDOV OEKJ & 10.95289 & 0 & 0 & 9 & 0 & 9 \\
\hline 83 & 1001 & 127 & KGRK OKBK & 13.44047 & 26.2 & 11.5 & 0 & 0 & 37.7 \\
\hline 83 & 1002 & 131 & KGRK $\sim \mathrm{OKBK}$ & 15.93037 & 26.2 & 11.5 & 0 & 0 & 37.7 \\
\hline 84 & 1008 & 51 & KLFI OKBK & 5.01605 & 10.7 & 16.3 & 0 & 0 & 27 \\
\hline 84 & 1009 & 81 & PHIK OKBK & 8.24425 & 4 & 41.38 & 0 & 0 & 45.38 \\
\hline 84 & 1010 & 109 & KGRK $\sim \mathrm{OKBK}$ & 10.84596 & 26.2 & 11.5 & 0 & 0 & 37.7 \\
\hline 84 & 1011 & 127 & KGRK $\sim \mathrm{OKBK}$ & 13.65632 & 26.2 & 11.5 & 0 & 0 & 37.7 \\
\hline 85 & 1020 & 36 & KDOV OKBK & 4.17615 & 0 & 0 & 2.4 & 0 & 2.4 \\
\hline 85 & 1020 & 38 & KDOV OKBK & 4.17615 & 0 & 0 & 15.2 & 0 & 15.2 \\
\hline 85 & 1020 & 23 & KDOV OKBK & 4.17615 & 0 & 0 & 0 & 29 & 5.8 \\
\hline 85 & 1021 & 81 & PHIK OKBK & 7.5176 & 0 & 4.72 & 5.8 & 0 & 10.52 \\
\hline 85 & 1022 & 109 & KGRK OKBK & 10.10206 & 26.2 & 11.5 & 0 & 0 & 37.7 \\
\hline
\end{tabular}




\begin{tabular}{|c|c|c|c|c|c|c|c|c|c|}
\hline & & & & & \multicolumn{5}{|c|}{-------DELIVERED QUANTITIES-------- } \\
\hline Tail\# & Msn\# & REQT\# & APOE $\sim$ APOD & ARR APOD & OUT & OVER & BULK & PAX & TOTAL \\
\hline 85 & 1023 & 127 & KGRK $\sim \mathrm{OKBK}$ & 12.59196 & 26.2 & 11.5 & 0 & 0 & 37.7 \\
\hline 86 & 1032 & 43 & KDMA OEKJ & 5.13639 & 11.9 & 33.67 & 0 & 29 & 51.37 \\
\hline 86 & 1033 & 82 & KBIF $\sim \mathrm{OKBK}$ & 8.3427 & 26.2 & 11.5 & 0 & 0 & 37.7 \\
\hline 86 & 1034 & 109 & KGRK $\sim \mathrm{OKBK}$ & 11.00723 & 26.2 & 11.5 & 0 & 0 & 37.7 \\
\hline 86 & 1035 & 127 & KGRK $\sim \mathrm{OKBK}$ & 13.61051 & 26.2 & 11.5 & 0 & 0 & 37.7 \\
\hline 87 & 1044 & 43 & KDMA OEKJ & 5.21654 & 0 & 41.9 & 0 & 28 & 47.5 \\
\hline 87 & 1046 & 109 & KGRK $\sim \mathrm{OKBK}$ & 10.86574 & 26.2 & 11.5 & 0 & 0 & 37.7 \\
\hline 87 & 1047 & 127 & KGRK $\sim \mathrm{OKBK}$ & 13.44727 & 26.2 & 11.5 & 0 & 0 & 37.7 \\
\hline 88 & 1056 & 43 & KDMA OEKJ & 5.13638 & 0 & 13.23 & 27.82 & 30 & 47.05 \\
\hline 88 & 1057 & 82 & KBIF $\sim \mathrm{OKBK}$ & 8.11675 & 33.9 & 5.2 & 0 & 0 & 39.1 \\
\hline 88 & 1058 & 109 & KGRK OKBK & 10.52657 & 26.2 & 11.5 & 0 & 0 & 37.7 \\
\hline 88 & 1059 & 127 & KGRK $\sim \mathrm{OKBK}$ & 13.11102 & 26.2 & 11.5 & 0 & 0 & 37.7 \\
\hline 89 & 1068 & 62 & KBIF $\sim \mathrm{OKBK}$ & 6.3427 & 26.2 & 11.5 & 0.1 & 0 & 37.8 \\
\hline 89 & 1069 & 70 & KDOV $\sim \mathrm{OKBK}$ & 8.77225 & 0 & 40.1 & 0 & 0 & 40.1 \\
\hline 89 & 1069 & 83 & KDOV OKBK & 8.77225 & 5 & 0 & 0 & 0 & 5 \\
\hline 89 & 1069 & 94 & KDOV OKBK & 8.77225 & 0 & 0.8 & 0 & 0 & 0.8 \\
\hline 89 & 1070 & 109 & KGRK $\sim \mathrm{OKBK}$ & 11.18207 & 26.2 & 11.5 & 0 & 0 & 37.7 \\
\hline 89 & 1071 & 127 & KGRK $\sim \mathrm{OKBK}$ & 13.59192 & 21.5 & 25.32 & 0 & 0 & 46.82 \\
\hline 90 & 1080 & 62 & KBIF OKBK & 6.17513 & 12 & 19 & 9.2 & 0 & 40.2 \\
\hline 90 & 1081 & 80 & KRIV OKBK & 9.02557 & 36.3 & 11.6 & 0 & 25 & 52.9 \\
\hline 90 & 1082 & 109 & KGRK OKBK & 12.19335 & 26.2 & 11.5 & 0 & 0 & 37.7 \\
\hline 90 & 1083 & 127 & KGRK $\sim \mathrm{OKBK}$ & 14.79674 & 0 & 47.9 & 0 & 0 & 47.9 \\
\hline 91 & 1093 & 15 & KHOP OKBK & 4.1756 & 0 & 6.1 & 4.9 & 15 & 14 \\
\hline 91 & 1092 & 33 & KBIF $\sim \mathrm{OBBS}$ & 6.76082 & 26.2 & 11.5 & 0 & 0 & 37.7 \\
\hline 91 & 1094 & 109 & KGRK $\sim \mathrm{OKBK}$ & 9.58355 & 26.2 & 11.5 & 0 & 0 & 37.7 \\
\hline 91 & 1095 & 127 & KGRK $\sim \mathrm{OKBK}$ & 12.33266 & 26.2 & 11.5 & 0 & 0 & 37.7 \\
\hline 92 & 1104 & 33 & $\mathrm{KBIF} \sim \mathrm{OBBS}$ & 4.3652 & 16.8 & 29 & 0 & 0 & 45.8 \\
\hline 92 & 1105 & 84 & KAEX $\sim \mathrm{OBBS}$ & 8.14136 & 26.2 & 11.5 & 0.1 & 0 & 37.8 \\
\hline 92 & 1106 & 109 & KGRK OKBK & 10.677 & 26.2 & 11.5 & 0 & 0 & 37.7 \\
\hline 92 & 1107 & 127 & KGRK $\sim \mathrm{OKBK}$ & 13.48737 & 26.2 & 11.5 & 0 & 0 & 37.7 \\
\hline 93 & 1116 & 33 & $\mathrm{KBIF} \sim \mathrm{OBBS}$ & 4.44537 & 0 & 47.9 & 0 & 0 & 47.9 \\
\hline 93 & 1117 & 60 & KBIF OBBS & 7.07635 & 26.2 & 11.5 & 0 & 0 & 37.7 \\
\hline 93 & 1118 & 109 & KGRK OKBK & 9.75324 & 26.2 & 11.5 & 0 & 0 & 37.7 \\
\hline 94 & 1128 & 80 & KRIV OKBK & 7.1682 & 0 & 40.1 & 0 & 22 & 44.5 \\
\hline 94 & 1129 & 129 & KSZL OKBK & 11.05884 & 26.2 & 10.5 & 0 & 0 & 36.7 \\
\hline 95 & 1140 & 104 & KGRK $\sim \mathrm{OKBK}$ & 9.07529 & 26.2 & 11.5 & 0 & 0 & 37.7 \\
\hline 95 & 1141 & 129 & KSZL $\sim \mathrm{OKBK}$ & 11.5322 & 41.3 & 0 & 0 & 2 & 41.7 \\
\hline 96 & 1152 & 104 & KGRK $\sim \mathrm{OKBK}$ & 9.15537 & 26.2 & 9.1 & 0 & 0 & 35.3 \\
\hline 96 & 1152 & 109 & KGRK $\sim \mathrm{OKBK}$ & 9.15537 & 0 & 2.4 & 0 & 0 & 2.4 \\
\hline 97 & 1165 & 80 & KRIV OKBK & 7.24841 & 36.3 & 11.6 & 0 & 25 & 52.9 \\
\hline 97 & 1164 & 104 & KGRK $\sim \mathrm{OKBK}$ & 9.65822 & 22.1 & 0 & 0 & 0 & 22.1 \\
\hline 97 & 1164 & 109 & KGRK OKBK & 9.65822 & 4.1 & 11.5 & 0 & 0 & 15.6 \\
\hline 98 & 1176 & 109 & KGRK OKBK & 9.15537 & 26.2 & 11.5 & 0 & 0 & 37.7 \\
\hline 99 & 1188 & 73 & KDOV OKBK & 7.02849 & 0 & 42.19 & 0 & 0 & 42.19 \\
\hline 99 & 1188 & 78 & KDOV OKBK & 7.02849 & 0 & 8.4 & 0 & 0 & 8.4 \\
\hline 100 & 1200 & 109 & KGRK $\sim \mathrm{OKBK}$ & 9.15537 & 26.2 & 11.5 & 0 & 0 & 37.7 \\
\hline 101 & 1212 & 109 & KGRK OKBK & 9.07529 & 26.2 & 11.5 & 0 & 0 & 37.7 \\
\hline 102 & 1224 & 109 & KGRK OKBK & 9.15537 & 26.2 & 11.5 & 0 & 0 & 37.7 \\
\hline 103 & 1236 & 109 & KGRK $\sim \mathrm{OKBK}$ & 9.07529 & 26.2 & 11.5 & 0 & 0 & 37.7 \\
\hline 104 & 1248 & 5 & KWRI OKBK & 1.4777 & 34.5 & 0 & 0 & 0 & 34.5 \\
\hline 104 & 1249 & 13 & KAGS OKBK & 4.62579 & 34.5 & 16.9 & 0 & 0 & 51.4 \\
\hline 104 & 1251 & 60 & $\mathrm{KBIF} \sim \mathrm{OBBS}$ & 7.80031 & 34.5 & 16.9 & 0 & 0 & 51.4 \\
\hline 104 & 1252 & 84 & KAEX OBBS & 14.08466 & 34.5 & 16.9 & 0 & 0 & 51.4 \\
\hline 105 & 1260 & 5 & KWRI OKBK & 1.4777 & 34.5 & 0 & 0 & 0 & 34.5 \\
\hline 105 & 1261 & 13 & KAGS OKBK & 5.08821 & 7.6 & 2.2 & 0 & 10 & 11.8 \\
\hline 105 & 1262 & 46 & KHMN OKBK & 8.23094 & 42.6 & 17 & 0 & 0 & 59.6 \\
\hline 105 & 1263 & 60 & KBIF $\sim \mathrm{OBBS}$ & 11.39576 & 18.6 & 30.5 & 0 & 0 & 49.1 \\
\hline 105 & 1264 & 84 & KAEX OBBS & 14.58199 & 34.5 & 16.9 & 0 & 0 & 51.4 \\
\hline
\end{tabular}




\begin{tabular}{|c|c|c|c|c|c|c|c|c|c|}
\hline & & & & & \multicolumn{5}{|c|}{--------DELIVERED QUANTITIES--------- } \\
\hline Tail\# & Msn\# & REQT\# & APOE APOD & ARR APOD & OUT & OVER & BULK & PAX & TOTAL \\
\hline 106 & 1272 & 5 & KWRI OKBK & 1.4777 & 34.5 & 0 & 0 & 0 & 34.5 \\
\hline 106 & 1274 & 46 & KHMN $\sim \mathrm{OKBK}$ & 5.21074 & 7.8 & 62.19 & 0.18 & 0 & 70.17 \\
\hline 106 & 1273 & 108 & $\mathrm{KCHS} \sim \mathrm{OBBI}$ & 11.519 & 29 & 13.9 & 0 & 8 & 44.5 \\
\hline 106 & 1276 & 120 & KDOV OKBK & 14.51051 & 8 & 0 & 0 & 0 & 8 \\
\hline 106 & 1276 & 124 & KDOV OKBK & 14.51051 & 21 & 0 & 0 & 0 & 21 \\
\hline 106 & 1277 & 131 & KGRK $\sim \mathrm{OKBK}$ & 17.72522 & 25.3 & 42.71 & 0 & 0 & 68.01 \\
\hline 107 & 1285 & 16 & KCHS OBBI & 2.11599 & 22.6 & 44.9 & 0 & 0 & 67.5 \\
\hline 107 & 1286 & 46 & KHMN $\sim \mathrm{OKBK}$ & 5.28542 & 42.6 & 17 & 0 & 0 & 59.6 \\
\hline 107 & 1287 & 58 & KPOB OKBK & 8.57833 & 0 & 19.7 & 0 & 0 & 19.7 \\
\hline 107 & 1287 & 59 & $\mathrm{KPOB} \sim \mathrm{OKBK}$ & 8.57833 & 19.4 & 36.01 & 0 & 0 & 55.41 \\
\hline 107 & 1284 & 111 & KHOP OKBK & 11.7212 & 0 & 59.23 & 3.11 & 0 & 62.35 \\
\hline 107 & 1288 & 125 & KDOV OKAJ & 14.85555 & 0 & 22 & 0 & 0 & 22 \\
\hline 107 & 1288 & 97 & KDOV OKAJ & 14.85555 & 0 & 0.9 & 0 & 0 & 0.9 \\
\hline 107 & 1289 & 131 & KGRK $\sim \mathrm{OKBK}$ & 18.16742 & 34.5 & 16.9 & 0 & 0 & 51.4 \\
\hline 108 & 1296 & 5 & KWRI OKBK & 1.41937 & 34.5 & 0 & 0 & 0 & 34.5 \\
\hline 108 & 1297 & 17 & KNGP $\sim \mathrm{OBBI}$ & 4.76019 & 15 & 0 & 0 & 8 & 16.6 \\
\hline 108 & 1297 & 8 & KNGP OBBI & 4.76019 & 0 & 0 & 0 & 48 & 9.6 \\
\hline 108 & 1298 & 46 & KHMN $\sim \mathrm{OKBK}$ & 8.07673 & 0 & 0 & 48.43 & 0 & 48.43 \\
\hline 108 & 1300 & 85 & KDMA OKBK & 14.36487 & 34.5 & 16.9 & 0 & 0 & 51.4 \\
\hline 108 & 1301 & 131 & KGRK $\sim \mathrm{OKBK}$ & 17.52116 & 34.5 & 16.9 & 0 & 0 & 51.4 \\
\hline 109 & 1308 & 7 & KDOV OBBS & 1.27498 & 29 & 0 & 0 & 0 & 29 \\
\hline 109 & 1309 & 8 & KNGP $\sim \mathrm{OBBI}$ & 4.48696 & 0 & 30.13 & 7 & 0 & 37.13 \\
\hline 109 & 1310 & 46 & KHMN $\sim \mathrm{OKBK}$ & 7.64224 & 42.6 & 17 & 0 & 0 & 59.6 \\
\hline 109 & 1312 & 85 & KDMA $\sim \mathrm{OKBK}$ & 10.79027 & 34.5 & 16.9 & 0 & 0 & 51.4 \\
\hline 109 & 1313 & 102 & KPOB OBBS & 13.95336 & 34.5 & 16.9 & 1.4 & 0 & 52.8 \\
\hline 109 & 1311 & 109 & KGRK $\sim \mathrm{OKBK}$ & 17.12206 & 34.5 & 16.9 & 0 & 0 & 51.4 \\
\hline 110 & 1320 & 7 & KDOV OBBS & 1.27498 & 29 & 0 & 0 & 0 & 29 \\
\hline 110 & 1321 & 17 & $\mathrm{KNGP} \sim \mathrm{OBBI}$ & 4.67687 & 29 & 10.4 & 0 & 0 & 39.4 \\
\hline 110 & 1322 & 46 & KHMN OKBK & 7.84175 & 0 & 32.21 & 37.29 & 0 & 69.5 \\
\hline 110 & 1324 & 85 & KDMA $\sim \mathrm{OKBK}$ & 11.14159 & 29.6 & 39.9 & 0 & 0 & 69.5 \\
\hline 110 & 1325 & 102 & $\mathrm{KPOB} \sim \mathrm{OBBS}$ & 14.45637 & 0.8 & 47.6 & 2.2 & 0 & 50.6 \\
\hline 110 & 1323 & 109 & KGRK $\sim \mathrm{OKBK}$ & 17.72747 & 34.5 & 16.9 & 0 & 0 & 51.4 \\
\hline 111 & 1332 & 7 & KDOV OBBS & 1.27498 & 29 & 0 & 0 & 0 & 29 \\
\hline 111 & 1333 & 25 & KHOP $\sim \mathrm{OEKJ}$ & 4.42335 & 0 & 0 & 45.42 & 0 & 45.42 \\
\hline 111 & 1334 & 48 & KMUO OTBD & 7.56801 & 0 & 63.14 & 0 & 0 & 63.14 \\
\hline 111 & 1336 & 85 & KDMA $\sim \mathrm{OKBK}$ & 10.74825 & 0 & 63.79 & 3.7 & 0 & 67.49 \\
\hline 111 & 1337 & 102 & KPOB OBBS & 13.91583 & 34.5 & 16.9 & 1.4 & 0 & 52.8 \\
\hline 111 & 1335 & 109 & $\mathrm{KGRK} \sim \mathrm{OKBK}$ & 17.2317 & 34.5 & 16.9 & 0 & 0 & 51.4 \\
\hline 112 & 1344 & 7 & KDOV OBBS & 1.42081 & 29 & 0 & 0 & 0 & 29 \\
\hline 112 & 1345 & 25 & KHOP $\sim \mathrm{OEKJ}$ & 5.01269 & 0 & 36.9 & 39.97 & 0 & 76.87 \\
\hline 112 & 1346 & 48 & KMUO OTBD & 8.30908 & 0 & 64.6 & 0 & 0 & 64.6 \\
\hline 112 & 1348 & 85 & KDMA $\mathrm{OKBK}$ & 11.64596 & 34.5 & 16.9 & 0 & 0 & 51.4 \\
\hline 112 & 1349 & 102 & KPOB $\sim \mathrm{OBBS}$ & 14.79938 & 34.5 & 16.9 & 1.4 & 0 & 52.8 \\
\hline 112 & 1347 & 109 & KGRK $\sim \mathrm{OKBK}$ & 17.97326 & 34.5 & 16.9 & 0 & 0 & 51.4 \\
\hline 113 & 1356 & 7 & KDOV OBBS & 1.42081 & 29 & 0 & 0 & 0 & 29 \\
\hline 113 & 1357 & 25 & KHOP $\sim \mathrm{OEKJ}$ & 4.57379 & 0 & 0 & 65.91 & 0 & 65.91 \\
\hline 113 & 1358 & 48 & KMUO OTBD & 7.72294 & 0 & 63.14 & 0 & 0 & 63.14 \\
\hline 113 & 1360 & 85 & KDMA $\sim \mathrm{OKBK}$ & 10.89857 & 34.5 & 16.9 & 0 & 0 & 51.4 \\
\hline 113 & 1361 & 102 & KPOB OBBS & 14.20369 & 34.5 & 16.9 & 1.4 & 0 & 52.8 \\
\hline 113 & 1359 & 109 & KGRK $\sim \mathrm{OKBK}$ & 17.38208 & 34.5 & 16.9 & 0 & 0 & 51.4 \\
\hline 114 & 1368 & 69 & KDOV OBBS & 9.27498 & 0 & 0 & 32.7 & 0 & 32.7 \\
\hline 114 & 1368 & 98 & KDOV OBBS & 9.27498 & 0 & 0 & 3.4 & 0 & 3.4 \\
\hline 114 & 1368 & 105 & KDOV OBBS & 9.27498 & 0 & 0 & 0 & 48 & 9.6 \\
\hline 114 & 1369 & 71 & $\mathrm{KGRK} \sim \mathrm{OKBK}$ & 12.45329 & 34.5 & 16.9 & 1.4 & 0 & 52.8 \\
\hline
\end{tabular}

Table A.2 Scenario 2 Mission Summary 


\section{A.3 SCENARIO 3 BEST SOLUTION MISSION SUMMARY}

\begin{tabular}{|c|c|c|c|c|c|c|c|c|c|}
\hline & & & & & -------D & LIVERED & QUANTIT & ES-----. & \\
\hline Tail\# & Msn\# & REQT\# & APOE $\sim$ APOD & ARR APOD & OUT & OVER & BULK & PAX & TOTAL \\
\hline 0 & 1 & 84 & KRCA RJBB & 15.395 & 0 & 0 & 0 & 334 & 66.8 \\
\hline 1 & 12 & 0 & KBLV LTAG & 0.86795 & 0 & 0 & 0 & 300 & 60 \\
\hline 2 & 24 & 11 & KELP $\sim \mathrm{OKBK}$ & 1.90573 & 0 & 0 & 0 & 8 & 1.6 \\
\hline 2 & 25 & 79 & KPHX RJSM & 16.46436 & 0 & 0 & 0 & 46 & 9.2 \\
\hline 3 & 36 & 10 & KEGI LTAG & 1.88039 & 0 & 0 & 0 & 335 & 67 \\
\hline 3 & 37 & 92 & KNZW RJSM & 16.46786 & 0 & 0 & 0 & 46 & 9.2 \\
\hline 4 & 48 & 10 & KEGI LTAG & 3.91505 & 0 & 0 & 0 & 335 & 67 \\
\hline 4 & 49 & 67 & KNZJ RJTA & 15.92786 & 0 & 0 & 0 & 120 & 24 \\
\hline 5 & 60 & 10 & KEGI LTAG & 3.05899 & 0 & 0 & 0 & 335 & 67 \\
\hline 5 & 61 & 61 & KNTU RJFF & 16.07507 & 0 & 0 & 0 & 123 & 24.6 \\
\hline 6 & 72 & 10 & KEGI LTAG & 4.20677 & 0 & 0 & 0 & 225 & 45 \\
\hline 6 & 73 & 88 & KNXX RJFK & 20.54683 & 0 & 0 & 0 & 28 & 5.6 \\
\hline 7 & 84 & 9 & KEDW LTAG & 2.85132 & 0 & 0 & 0 & 28 & 5.6 \\
\hline 7 & 85 & 73 & KOQU RJCJ & 20.02224 & 0 & 0 & 0 & 291 & 58.2 \\
\hline 8 & 96 & 7 & KDSM LTAG & 3.3305 & 0 & 0 & 0 & 335 & 67 \\
\hline 8 & 97 & 73 & KOQU RJCJ & 21.81772 & 0 & 0 & 0 & 335 & 67 \\
\hline 9 & 108 & 7 & KDSM LTAG & 3.05778 & 0 & 0 & 0 & 335 & 67 \\
\hline 9 & 109 & 73 & KOQU RJCJ & 24.02677 & 0 & 0 & 0 & 335 & 67 \\
\hline 10 & 120 & 7 & KDSM LTAG & 3.62226 & 0 & 0 & 0 & 335 & 67 \\
\hline 10 & 121 & 73 & KOQU RJCJ & 20.72105 & 0 & 0 & 0 & 335 & 67 \\
\hline 21 & 252 & 7 & KDSM LTAG & 3.92085 & 0 & 0 & 0 & 335 & 67 \\
\hline 21 & 253 & 73 & KOQU RJCJ & 22.92615 & 0 & 0 & 0 & 260 & 52 \\
\hline 22 & 264 & 7 & KDSM LTAG & 3.47634 & 0 & 0 & 0 & 335 & 67 \\
\hline 22 & 265 & 73 & KOQU RJCJ & 19.68552 & 0 & 0 & 0 & 144 & 28.8 \\
\hline 23 & 276 & 7 & KDSM LTAG & 2.03687 & 0 & 0 & 0 & 25 & 5 \\
\hline 23 & 277 & 71 & KOFF $\sim$ RJBB & 23.48611 & 0 & 0 & 0 & 335 & 67 \\
\hline 24 & 289 & 71 & KOFF RJBB & 22.09189 & 0 & 0 & 0 & 65 & 13 \\
\hline 25 & 300 & 57 & KNKT $\sim$ RJAA & 5.08951 & 0 & 0 & 0 & 322 & 64.4 \\
\hline 25 & 301 & 56 & KSTJ $\sim \mathrm{OMAD}$ & 22.87553 & 0 & 0 & 0 & 133 & 26.6 \\
\hline 26 & 312 & 25 & KGFA LTAG & 6.09981 & 0 & 0 & 0 & 65 & 13 \\
\hline 26 & 313 & 82 & KPOB RJTY & 24.33188 & 0 & 0 & 0 & 123 & 24.6 \\
\hline 27 & 325 & 80 & KPIA RJTA & 24.65623 & 0 & 0 & 0 & 65 & 13 \\
\hline 28 & 336 & 21 & KFSI $\sim$ LTAG & 4.9026 & 0 & 0 & 0 & 335 & 67 \\
\hline 28 & 337 & 76 & KPBG $\sim \mathrm{RJFU}$ & 27.65861 & 0 & 0 & 0 & 286 & 57.2 \\
\hline 29 & 348 & 21 & KFSI LTAG & 6.07618 & 0 & 0 & 0 & 6 & 1.2 \\
\hline 29 & 349 & 76 & KPBG RJFU & 26.53951 & 0 & 0 & 0 & 252 & 50.4 \\
\hline 30 & 360 & 20 & KFSD LTAG & 5.47274 & 0 & 0 & 0 & 335 & 67 \\
\hline 30 & 361 & 76 & KPBG $\sim$ RJFU & 26.72147 & 0 & 0 & 0 & 335 & 67 \\
\hline 41 & 492 & 20 & KFSD LTAG & 7.19636 & 0 & 0 & 0 & 195 & 39 \\
\hline 41 & 493 & 76 & KPBG $\sim$ RJFU & 27.55908 & 0 & 0 & 0 & 195 & 39 \\
\hline 42 & 504 & 20 & KFSD LTAG & 8.52832 & 0 & 0 & 0 & 165 & 33 \\
\hline 42 & 505 & 76 & KPBG RJFU & 27.56455 & 0 & 0 & 0 & 162 & 32.4 \\
\hline 43 & 516 & 19 & KFOK $\sim \mathrm{LTAG}$ & 4.64694 & 0 & 0 & 0 & 195 & 39 \\
\hline 43 & 517 & 69 & KNZY RJTY & 26.0851 & 0 & 0 & 0 & 195 & 39 \\
\hline 44 & 528 & 19 & KFOK $\sim \mathrm{LTAG}$ & 6.37736 & 0 & 0 & 0 & 49 & 9.8 \\
\hline 44 & 529 & 69 & KNZY RJTY & 25.14247 & 0 & 0 & 0 & 114 & 22.8 \\
\hline 45 & 540 & 16 & KFLL LTAG & 8.36564 & 0 & 0 & 0 & 123 & 24.6 \\
\hline 45 & 541 & 93 & KNZY RJTA & 25.31842 & 0 & 0 & 0 & 65 & 13 \\
\hline 46 & 552 & 14 & KFFO LTAG & 4.67095 & 0 & 0 & 0 & 65 & 13 \\
\hline 46 & 553 & 89 & KNYL RJFU & 26.46359 & 0 & 0 & 0 & 195 & 39 \\
\hline 47 & 564 & 13 & KFAR OMAD & 4.86241 & 0 & 0 & 0 & 46 & 9.2 \\
\hline 47 & 565 & 89 & KNYL RJFU & 24.77759 & 0 & 0 & 0 & 195 & 39 \\
\hline 48 & 577 & 89 & KNYL RJFU & 25.05778 & 0 & 0 & 0 & 195 & 39 \\
\hline
\end{tabular}




\begin{tabular}{|c|c|c|c|c|c|c|c|c|c|}
\hline \multirow[b]{2}{*}{ Tail\# } & \multirow[b]{2}{*}{ Msn\# } & \multirow[b]{2}{*}{ REQT\# } & \multirow[b]{2}{*}{ APOE $\sim$ APOD } & \multirow[b]{2}{*}{ ARR APOD } & \multicolumn{5}{|c|}{-----DELIVERED QUANTITIES------- } \\
\hline & & & & & OUT & OVER & BULK & PAX & TOTAL \\
\hline 49 & 588 & 28 & KGSB LTAY & 5.64383 & 0 & 0 & 0 & 76 & 15.2 \\
\hline 49 & 589 & 89 & KNYL RJFU & 25.73609 & 0 & 0 & 0 & 195 & 39 \\
\hline 50 & 601 & 89 & KNYL RJFU & 25.50948 & 0 & 0 & 0 & 195 & 39 \\
\hline 51 & 612 & 41 & KNGZ LTAG & 11.32473 & 0 & 0 & 0 & 195 & 39 \\
\hline 51 & 613 & 89 & KNYL RJFU & 26.68171 & 0 & 0 & 0 & 195 & 39 \\
\hline 52 & 624 & 41 & KNGZ LTAG & 11.18595 & 0 & 0 & 0 & 183 & 36.6 \\
\hline 52 & 625 & 89 & KNYL RJFU & 26.0257 & 0 & 0 & 0 & 60 & 12 \\
\hline 53 & 637 & 44 & KWRI ETAR & 1.80037 & 0 & 0 & 0 & 106 & 21.2 \\
\hline 53 & 636 & 35 & KNGU LTCC & 7.66526 & 0 & 0 & 0 & 4 & 0.8 \\
\hline 54 & 648 & 39 & KNGP LTAC & 7.73625 & 0 & 0 & 0 & 89 & 17.8 \\
\hline 55 & 660 & 33 & KNFW LTBL & 8.72817 & 0 & 0 & 0 & 2 & 0.4 \\
\hline 55 & 661 & 90 & KNZC $\sim$ RJNN & 27.23256 & 0 & 0 & 0 & 8 & 1.6 \\
\hline 56 & 672 & 38 & KNFW LTAG & 9.36275 & 0 & 0 & 0 & 123 & 24.6 \\
\hline 56 & 673 & 95 & KDOV RJTY & 27.29539 & 0 & 0 & 0 & 123 & 24.6 \\
\hline 57 & 684 & 23 & KFWA LTAG & 9.27807 & 0 & 0 & 0 & 43 & 8.6 \\
\hline 59 & 708 & 34 & KNGP LTBU & 10.44629 & 0 & 0 & 0 & 195 & 39 \\
\hline 60 & 720 & 34 & KNGP $\sim$ LTBU & 9.74103 & 0 & 0 & 0 & 127 & 25.4 \\
\hline 61 & 732 & 46 & KDOV LTAG & 11.84901 & 0 & 0 & 0 & 195 & 39 \\
\hline 62 & 744 & 46 & KDOV LTAG & 11.68367 & 0 & 0 & 0 & 114 & 22.8 \\
\hline 63 & 756 & 54 & KSPS OKBK & 10.14593 & 0 & 0 & 0 & 12 & 2.4 \\
\hline 64 & 768 & 77 & KPDX RJNN & 11.52648 & 0 & 0 & 0 & 8 & 1.6 \\
\hline 85 & 1020 & 86 & KBLV RJCJ & 11.64207 & 0 & 0 & 0 & 195 & 39 \\
\hline 86 & 1032 & 86 & KBLV RJCJ & 13.15766 & 0 & 0 & 0 & 195 & 39 \\
\hline 87 & 1044 & 86 & KBLV RJCJ & 14.29984 & 0 & 0 & 0 & 195 & 39 \\
\hline 88 & 1056 & 86 & KBLV RJCJ & 12.34679 & 0 & 0 & 0 & 195 & 39 \\
\hline 89 & 1068 & 86 & KBLV RJCJ & 13.83495 & 0 & 0 & 0 & 195 & 39 \\
\hline 90 & 1080 & 86 & KBLV RJCJ & 13.50464 & 0 & 0 & 0 & 195 & 39 \\
\hline 91 & 1092 & 86 & KBLV RJCJ & 12.66291 & 0 & 0 & 0 & 195 & 39 \\
\hline 92 & 1104 & 86 & KBLV RJCJ & 12.86599 & 0 & 0 & 0 & 195 & 39 \\
\hline 93 & 1116 & 86 & KBLV RJCJ & 13.27887 & 0 & 0 & 0 & 140 & 28 \\
\hline 94 & 1128 & 51 & KSKF $\sim$ TAG & 14.37899 & 0 & 0 & 0 & 195 & 39 \\
\hline 95 & 1140 & 51 & KSKF LTAG & 12.19858 & 0 & 0 & 0 & 195 & 39 \\
\hline 96 & 1152 & 51 & KSKF LTAG & 14.08717 & 0 & 0 & 0 & 195 & 39 \\
\hline 97 & 1164 & 51 & KSKF LTAG & 11.9192 & 0 & 0 & 0 & 195 & 39 \\
\hline 98 & 1176 & 51 & KSKF LTAG & 14.08733 & 0 & 0 & 0 & 195 & 39 \\
\hline 99 & 1188 & 51 & KSKF $\sim$ LTAG & 12.78171 & 0 & 0 & 0 & 29 & 5.8 \\
\hline 101 & 1212 & 58 & KNKX $\sim \mathrm{RJBB}$ & 13.43142 & 0 & 0 & 0 & 4 & 0.8 \\
\hline 102 & 1224 & 75 & KPAM RJFK & 16.26572 & 0 & 0 & 0 & 28 & 5.6 \\
\hline 103 & 1236 & 64 & KNXX RJNN & 15.22421 & 0 & 0 & 0 & 195 & 39 \\
\hline 104 & 1248 & 64 & KNXX RJNN & 15.2444 & 0 & 0 & 0 & 195 & 39 \\
\hline 105 & 1260 & 64 & KNXX RJNN & 14.69913 & 0 & 0 & 0 & 195 & 39 \\
\hline 106 & 1272 & 64 & KNXX RJNN & 14.88481 & 0 & 0 & 0 & 93 & 18.6 \\
\hline 107 & 1284 & 48 & KSFO OJ2X & 14.85944 & 0 & 0 & 0 & 195 & 39 \\
\hline 108 & 1296 & 48 & $\mathrm{KSFO} \sim \mathrm{OJ} 2 \mathrm{X}$ & 14.99448 & 0 & 0 & 0 & 195 & 39 \\
\hline 109 & 1308 & 48 & KSFO OJ2X & 15.14198 & 0 & 0 & 0 & 10 & 2 \\
\hline 135 & 1620 & 6 & KDPG OJHF & 1.68804 & 0 & 0 & 87.4 & 0 & 87.4 \\
\hline 135 & 1622 & 72 & KOKC $\sim$ RJCC & 21.00367 & 0 & 0 & 78.66 & 0 & 78.66 \\
\hline 135 & 1623 & 94 & KBLV RJTT & 29.59636 & 0 & 0 & 87.4 & 0 & 87.4 \\
\hline 136 & 1632 & 6 & KDPG $\sim \mathrm{OJHF}$ & 1.24845 & 0 & 0 & 87.4 & 0 & 87.4 \\
\hline 136 & 1633 & 55 & KSSC $\sim$ OMAA & 12.06317 & 0 & 0 & 7.9 & 0 & 7.9 \\
\hline 136 & 1634 & 72 & KOKC $\sim \mathrm{RJCC}$ & 20.81976 & 0 & 0 & 71.76 & 0 & 71.76 \\
\hline 136 & 1635 & 94 & KBLV RJTT & 30.28236 & 0 & 0 & 87.4 & 0 & 87.4 \\
\hline 137 & 1644 & 6 & KDPG OJHF & 1.84268 & 0 & 0 & 87.4 & 0 & 87.4 \\
\hline 137 & 1645 & 51 & KSKF $\sim$ LTAG & 12.98052 & 0 & 0 & 87.4 & 0 & 87.4 \\
\hline 137 & 1646 & 72 & KOKC $\sim \mathrm{RJCC}$ & 21.53155 & 0 & 0 & 87.4 & 0 & 87.4 \\
\hline 137 & 1647 & 94 & KBLV RJTT & 30.17782 & 0 & 0 & 87.4 & 0 & 87.4 \\
\hline 138 & 1656 & 6 & KDPG OJHF & 1.53974 & 0 & 0 & 38.4 & 0 & 38.4 \\
\hline 138 & 1657 & 51 & KSKF LTAG & 12.45259 & 0 & 0 & 87.4 & 0 & 87.4 \\
\hline
\end{tabular}




\begin{tabular}{|c|c|c|c|c|c|c|c|c|c|}
\hline & & & & & ----DE & LIVERED & QUANTIT & ES------- & \\
\hline Tail\# & Msn\# & REQT\# & APOE $\sim$ APOD & ARR APOD & $\begin{array}{l}\text { OUT } \\
\end{array}$ & OVER & BULK & PAX & TOTAL \\
\hline 138 & 1658 & 72 & KOKC $\sim$ RJCC & 24.06594 & 0 & 0 & 87.4 & 0 & 87.4 \\
\hline 138 & 1659 & 94 & KBLV RJTT & 32.10397 & 0 & 0 & 65.53 & 0 & 65.53 \\
\hline 139 & 1668 & 4 & KDNL OJ1X & 1.49362 & 0 & 0 & 87.4 & 0 & 87.4 \\
\hline 139 & 1669 & 51 & KSKF $\sim$ LTAG & 11.99624 & 0 & 0 & 87.4 & 0 & 87.4 \\
\hline 139 & 1670 & 72 & KOKC $\sim$ RJCC & 24.13377 & 0 & 0 & 87.4 & 0 & 87.4 \\
\hline 139 & 1671 & 94 & KBLV RJTT & 26.93349 & 0 & 0 & 15.07 & 0 & 15.07 \\
\hline 140 & 1680 & 4 & KDNL OJ1X & 1.2023 & 0 & 0 & 87.4 & 0 & 87.4 \\
\hline 140 & 1681 & 51 & KSKF $\sim$ LTAG & 12.00315 & 0 & 0 & 87.4 & 0 & 87.4 \\
\hline 140 & 1682 & 72 & KOKC $\sim$ RJCC & 22.99011 & 0 & 0 & 65.53 & 0 & 65.53 \\
\hline 141 & 1692 & 4 & KDNL OJ1X & 1.20268 & 0 & 0 & 87.4 & 0 & 87.4 \\
\hline 141 & 1693 & 51 & KSKF $\sim$ LTAG & 13.70969 & 0 & 0 & 87.4 & 0 & 87.4 \\
\hline 141 & 1694 & 72 & KOKC $\sim$ RJCC & 21.20784 & 0 & 0 & 78.66 & 0 & 78.66 \\
\hline 142 & 1705 & 51 & KSKF $\sim$ LTAG & 12.30664 & 0 & 0 & 18.4 & 0 & 18.4 \\
\hline 142 & 1706 & 72 & KOKC $\sim$ RJCC & 20.81976 & 0 & 0 & 71.76 & 0 & 71.76 \\
\hline 143 & 1716 & 1 & KDLF LTAG & 1.0441 & 0 & 0 & 23.5 & 0 & 23.5 \\
\hline 143 & 1717 & 49 & KSGH OJHF & 13.99031 & 0 & 0 & 72.2 & 0 & 72.2 \\
\hline 143 & 1718 & 72 & KOKC $\sim$ RJCC & 22.11488 & 0 & 0 & 87.4 & 0 & 87.4 \\
\hline 144 & 1728 & 9 & KEDW LTAG & 4.3702 & 0 & 0 & 45.5 & 0 & 45.5 \\
\hline 144 & 1729 & 49 & KSGH OJHF & 15.10931 & 0 & 0 & 72.2 & 0 & 72.2 \\
\hline 144 & 1730 & 72 & KOKC $\sim \mathrm{RJCC}$ & 24.15344 & 0 & 0 & 87.4 & 0 & 87.4 \\
\hline 145 & 1740 & 3 & KDMA LTAG & 2.73378 & 0 & 0 & 13 & 0 & 13 \\
\hline 145 & 1741 & 49 & KSGH OJHF & 13.79921 & 0 & 0 & 72.2 & 0 & 72.2 \\
\hline 145 & 1742 & 72 & KOKC $\sim$ RJCC & 22.50043 & 0 & 0 & 87.4 & 0 & 87.4 \\
\hline 146 & 1752 & 60 & KNTD RJCJ & 4.60994 & 0 & 0 & 23.2 & 0 & 23.2 \\
\hline 146 & 1753 & 49 & KSGH $\sim \mathrm{OJHF}$ & 12.19296 & 0 & 0 & 72.2 & 0 & 72.2 \\
\hline 146 & 1754 & 72 & KOKC $\sim \mathrm{RJCC}$ & 23.16511 & 0 & 0 & 65.53 & 0 & 65.53 \\
\hline 147 & 1764 & 21 & KFSI LTAG & 5.23555 & 0 & 0 & 10.2 & 0 & 10.2 \\
\hline 147 & 1765 & 49 & KSGH $\sim \mathrm{OJHF}$ & 14.13614 & 0 & 0 & 72.2 & 0 & 72.2 \\
\hline 147 & 1766 & 72 & KOKC $\sim$ RJCC & 21.20784 & 0 & 0 & 78.66 & 0 & 78.66 \\
\hline 148 & 1776 & 15 & KFHU LTAG & 7.62224 & 0 & 0 & 87.4 & 0 & 87.4 \\
\hline 148 & 1777 & 49 & KSGH OJHF & 14.23431 & 0 & 0 & 72.2 & 0 & 72.2 \\
\hline 148 & 1778 & 72 & KOKC $\sim$ RJCC & 20.81976 & 0 & 0 & 71.76 & 0 & 71.76 \\
\hline 149 & 1788 & 15 & KFHU LTAG & 8.06666 & 0 & 0 & 87.4 & 0 & 87.4 \\
\hline 149 & 1789 & 49 & KSGH OJHF & 13.79921 & 0 & 0 & 72.2 & 0 & 72.2 \\
\hline 149 & 1790 & 72 & KOKC $\sim \mathrm{RJCC}$ & 22.11488 & 0 & 0 & 87.4 & 0 & 87.4 \\
\hline 150 & 1800 & 15 & KFHU LTAG & 6.74715 & 0 & 0 & 87.4 & 0 & 87.4 \\
\hline 150 & 1801 & 49 & KSGH OJHF & 11.90129 & 0 & 0 & 72.2 & 0 & 72.2 \\
\hline 150 & 1802 & 72 & KOKC $\sim \mathrm{RJCC}$ & 23.86177 & 0 & 0 & 87.4 & 0 & 87.4 \\
\hline 151 & 1812 & 15 & KFHU LTAG & 6.76609 & 0 & 0 & 80.6 & 0 & 80.6 \\
\hline 151 & 1813 & 49 & KSGH $\sim \mathrm{OJHF}$ & 15.01114 & 0 & 0 & 72.2 & 0 & 72.2 \\
\hline 151 & 1814 & 72 & KOKC $\sim$ RJCC & 23.63793 & 0 & 0 & 87.4 & 0 & 87.4 \\
\hline 152 & 1824 & 29 & KGSP LTAZ & 5.75363 & 0 & 0 & 34.5 & 0 & 34.5 \\
\hline 152 & 1825 & 49 & KSGH $\sim \mathrm{OJHF}$ & 14.81764 & 0 & 0 & 72.2 & 0 & 72.2 \\
\hline 152 & 1826 & 72 & KOKC $\sim$ RJCC & 23.22344 & 0 & 0 & 65.53 & 0 & 65.53 \\
\hline 153 & 1836 & 27 & KGRK LTAG & 10.91694 & 0 & 0 & 17.8 & 0 & 17.8 \\
\hline 153 & 1837 & 49 & KSGH OJHF & 12.68436 & 0 & 0 & 65.8 & 0 & 65.8 \\
\hline 153 & 1838 & 72 & KOKC $\sim$ RJCC & 21.20784 & 0 & 0 & 78.66 & 0 & 78.66 \\
\hline 154 & 1848 & 26 & KGPT LTAG & 10.29882 & 0 & 0 & 87.4 & 0 & 87.4 \\
\hline 154 & 1849 & 58 & KNKX RJBB & 13.53935 & 0 & 0 & 72.2 & 0 & 72.2 \\
\hline 154 & 1850 & 66 & KNZC $\sim$ RJSM & 24.02373 & 0 & 0 & 8.9 & 0 & 8.9 \\
\hline 182 & 2184 & 26 & KGPT $\sim$ LTAG & 8.99324 & 0 & 0 & 87.4 & 0 & 87.4 \\
\hline 182 & 2185 & 58 & KNKX RJBB & 13.66761 & 0 & 0 & 18.2 & 0 & 18.2 \\
\hline 182 & 2186 & 85 & KHIF RJCC & 21.26461 & 0 & 0 & 71.76 & 0 & 71.76 \\
\hline 183 & 2196 & 26 & KGPT $\sim$ LTAG & 10.00706 & 0 & 0 & 87.4 & 0 & 87.4 \\
\hline 183 & 2197 & 75 & KPAM RJFK & 17.72846 & 0 & 0 & 85.03 & 0 & 85.03 \\
\hline 183 & 2198 & 85 & KHIF RJCC & 20.78976 & 0 & 0 & 87.4 & 0 & 87.4 \\
\hline 184 & 2208 & 26 & KGPT $\sim$ LTAG & 8.7135 & 0 & 0 & 87.4 & 0 & 87.4 \\
\hline 184 & 2209 & 75 & KPAM RJFK & 18.6031 & 0 & 0 & 87.4 & 0 & 87.4 \\
\hline
\end{tabular}




\begin{tabular}{|c|c|c|c|c|c|c|c|c|c|}
\hline & & & & & -----DI & IVERED & QUANTIT & ES------- & \\
\hline Tail\# & Msn\# & REQT\# & APOE $\sim$ APOD & ARR APOD & OUT & OVER & BULK & $\overline{P A X}$ & TOTAL \\
\hline 184 & 2210 & 85 & KHIF RJCC & 20.94516 & 0 & 0 & 87.4 & 0 & 87.4 \\
\hline 185 & 2220 & 26 & KGPT $\sim$ LTAG & 8.98626 & 0 & 0 & 50.8 & 0 & 50.8 \\
\hline 185 & 2221 & 75 & KPAM RJFK & 16.60288 & 0 & 0 & 65.53 & 0 & 65.53 \\
\hline 185 & 2222 & 85 & KHIF RJCC & 22.22584 & 0 & 0 & 87.4 & 0 & 87.4 \\
\hline 186 & 2232 & 37 & KNHK LTFJ & 7.6879 & 0 & 0 & 23.2 & 0 & 23.2 \\
\hline 186 & 2233 & 75 & KPAM RJFK & 14.78355 & 0 & 0 & 69.05 & 0 & 69.05 \\
\hline 186 & 2234 & 85 & KHIF RJCC & 21.86917 & 0 & 0 & 65.53 & 0 & 65.53 \\
\hline 187 & 2244 & 35 & KNGU LTCC & 7.75271 & 0 & 0 & 82.7 & 0 & 82.7 \\
\hline 187 & 2245 & 75 & KPAM RJFK & 18.44862 & 0 & 0 & 38.49 & 0 & 38.49 \\
\hline 187 & 2246 & 85 & KHIF RJCC & 20.73585 & 0 & 0 & 78.66 & 0 & 78.66 \\
\hline 188 & 2256 & 35 & KNGU LTCC & 8.74857 & 0 & 0 & 7.7 & 0 & 7.7 \\
\hline 188 & 2257 & 53 & KSPI LTAG & 15.04617 & 0 & 0 & 87.4 & 0 & 87.4 \\
\hline 188 & 2258 & 85 & KHIF $\sim$ RJCC & 21.61461 & 0 & 0 & 71.76 & 0 & 71.76 \\
\hline 189 & 2268 & 33 & KNFW LTBL & 7.77132 & 0 & 0 & 20.3 & 0 & 20.3 \\
\hline 189 & 2269 & 53 & KSPI LTAG & 14.77345 & 0 & 0 & 87.4 & 0 & 87.4 \\
\hline 189 & 2270 & 85 & KHIF RJCC & 21.1033 & 0 & 0 & 87.4 & 0 & 87.4 \\
\hline 190 & 2280 & 38 & KNFW LTAG & 11.44538 & 0 & 0 & 5.6 & 0 & 5.6 \\
\hline 190 & 2281 & 53 & KSPI LTAG & 18.10876 & 0 & 0 & 87.4 & 0 & 87.4 \\
\hline 190 & 2282 & 85 & KHIF $\sim$ RJCC & 20.94516 & 0 & 0 & 87.4 & 0 & 87.4 \\
\hline 191 & 2292 & 23 & KFWA LTAG & 10.51364 & 0 & 0 & 72.2 & 0 & 72.2 \\
\hline 191 & 2293 & 53 & KSPI LTAG & 18.26144 & 0 & 0 & 60.1 & 0 & 60.1 \\
\hline 191 & 2294 & 85 & KHIF $\sim$ RJCC & 22.22584 & 0 & 0 & 87.4 & 0 & 87.4 \\
\hline 192 & 2304 & 23 & KFWA LTAG & 9.92332 & 0 & 0 & 72.2 & 0 & 72.2 \\
\hline 192 & 2305 & 84 & KRCA RJBB & 15.31523 & 0 & 0 & 56.9 & 0 & 56.9 \\
\hline 192 & 2306 & 85 & KHIF RJCC & 21.86917 & 0 & 0 & 65.53 & 0 & 65.53 \\
\hline 193 & 2316 & 23 & KFWA LTAG & 10.23393 & 0 & 0 & 72.2 & 0 & 72.2 \\
\hline 193 & 2317 & 61 & KNTU RJFF & 16.01875 & 0 & 0 & 5.6 & 0 & 5.6 \\
\hline 193 & 2318 & 85 & KHIF RJCC & 20.73585 & 0 & 0 & 78.66 & 0 & 78.66 \\
\hline 194 & 2328 & 23 & KFWA LTAG & 10.79842 & 0 & 0 & 72.2 & 0 & 72.2 \\
\hline 194 & 2330 & 85 & KHIF RJCC & 21.26461 & 0 & 0 & 71.76 & 0 & 71.76 \\
\hline 195 & 2340 & 23 & KFWA LTAG & 10.07614 & 0 & 0 & 72.2 & 0 & 72.2 \\
\hline 195 & 2341 & 81 & KPIT RJTT & 19.10431 & 0 & 0 & 87.4 & 0 & 87.4 \\
\hline 195 & 2342 & 85 & KHIF $\sim$ RJCC & 24.30202 & 0 & 0 & 87.4 & 0 & 87.4 \\
\hline 196 & 2352 & 23 & KFWA LTAG & 10.94415 & 0 & 0 & 72.2 & 0 & 72.2 \\
\hline 196 & 2353 & 81 & KPIT RJTT & 19.21485 & 0 & 0 & 87.4 & 0 & 87.4 \\
\hline 196 & 2354 & 85 & KHIF $\sim$ RJCC & 22.64503 & 0 & 0 & 87.4 & 0 & 87.4 \\
\hline 212 & 2544 & 5 & KDOV OJ2X & 0.98792 & 0 & 30.9 & 0 & 5 & 31.9 \\
\hline 212 & 2545 & 45 & KWRI ETAR & 9.52122 & 37.9 & 0 & 0 & 0 & 37.9 \\
\hline 212 & 2547 & 62 & KNUQ RJFK & 26.1763 & 18 & 0 & 0 & 0 & 18 \\
\hline 212 & 2546 & 62 & KNUQ RJFK & 29.12746 & 18 & 0 & 0 & 0 & 18 \\
\hline 213 & 2556 & 4 & $\overline{\mathrm{KDNL} \sim \mathrm{OJ} 1 \mathrm{X}}$ & 1.81475 & 13 & 0 & 17.8 & 0 & 30.8 \\
\hline 213 & 2557 & 65 & KNYL RJOI & 12.25285 & 15.5 & 0 & 0 & 0 & 15.5 \\
\hline 213 & 2558 & 85 & KHIF RJCC & 22.36077 & 0 & 0 & 41.4 & 0 & 41.4 \\
\hline 213 & 2559 & 62 & KNUQ RJFK & 26.53157 & 18 & 0 & 0 & 0 & 18 \\
\hline 214 & 2568 & 2 & KDLH $\sim$ LTAG & 1.45243 & 0 & 18.1 & 0 & 0 & 18.1 \\
\hline 214 & 2570 & 85 & KHIF RJCC & 21.76609 & 0 & 0 & 41.4 & 0 & 41.4 \\
\hline 214 & 2571 & 62 & KNUQ RJFK & 26.7386 & 18 & 0 & 0 & 0 & 18 \\
\hline 214 & 2569 & 62 & KNUQ RJFK & 29.98135 & 18 & 0 & 0 & 0 & 18 \\
\hline 215 & 2580 & 1 & KDLF LTAG & 1.55629 & 3.2 & 0 & 0 & 0 & 3.2 \\
\hline 215 & 2582 & 85 & KHIF RJCC & 21.79944 & 0 & 0 & 41.4 & 0 & 41.4 \\
\hline 215 & 2583 & 62 & KNUQ RJFK & 25.00877 & 18 & 0 & 0 & 0 & 18 \\
\hline 215 & 2581 & 62 & KNUQ RJFK & 28.55904 & 18 & 0 & 0 & 0 & 18 \\
\hline 216 & 2592 & 0 & KBLV $\sim$ LTAG & 1.71165 & 0 & 3.5 & 0 & 0 & 3.5 \\
\hline 216 & 2593 & 63 & KNUW RJFU & 12.13073 & 0 & 58.28 & 0 & 0 & 58.28 \\
\hline 216 & 2594 & 85 & KHIF RJCC & 23.92325 & 0 & 0 & 41.4 & 0 & 41.4 \\
\hline 217 & 2604 & 9 & KEDW LTAG & 4.38146 & 0.5 & 11.2 & 0 & 0 & 11.7 \\
\hline 217 & 2605 & 63 & KNUW RJFU & 12.33911 & 0 & 55.54 & 0 & 0 & 55.54 \\
\hline 217 & 2606 & 85 & KHIF RJCC & 22.67362 & 0 & 0 & 41.4 & 0 & 41.4 \\
\hline
\end{tabular}




\begin{tabular}{|c|c|c|c|c|c|c|c|c|c|}
\hline & & & & & -------D & LIVERED & QUANTIT & ES----- & \\
\hline Tail\# & Msn\# & REQT\# & APOE $\sim$ APOD & ARR APOD & OUT & OVER & BULK & PAX & TOTAL \\
\hline 217 & 2607 & 62 & KNUQ RJFK & 25.99958 & 18 & 0 & 0 & 0 & 18 \\
\hline 218 & 2616 & 8 & KDYS LTAG & 3.28333 & 37.2 & 0 & 0 & 0 & 37.2 \\
\hline 218 & 2617 & 63 & KNUW $\sim$ RJFU & 11.95741 & 0 & 65.53 & 0 & 0 & 65.53 \\
\hline 218 & 2619 & 83 & KPSM RJAA & 18.67012 & 34.5 & 0 & 0 & 0 & 34.5 \\
\hline 218 & 2618 & 85 & KHIF $\sim$ RJCC & 23.36537 & 0 & 0 & 41.4 & 0 & 41.4 \\
\hline 219 & 2628 & 7 & KDSM LTAG & 3.67613 & 0 & 6.6 & 0 & 0 & 6.6 \\
\hline 219 & 2629 & 63 & KNUW RJFU & 11.80072 & 0 & 72.71 & 0 & 0 & 72.71 \\
\hline 219 & 2630 & 85 & KHIF RJCC & 22.02129 & 0 & 0 & 41.4 & 0 & 41.4 \\
\hline 219 & 2631 & 62 & KNUQ $\sim$ RJFK & 25.8636 & 18 & 0 & 0 & 0 & 18 \\
\hline 220 & 2640 & 44 & KWRI ETAR & 1.68157 & 0 & 14.5 & 0 & 14 & 17.3 \\
\hline 220 & 2641 & 63 & KNUW RJFU & 12.36338 & 0 & 58.35 & 0 & 0 & 58.35 \\
\hline 220 & 2642 & 85 & KHIF $\sim \mathrm{RJCC}$ & 21.55882 & 0 & 0 & 41.4 & 0 & 41.4 \\
\hline 220 & 2643 & 62 & KNUQ RJFK & 25.30044 & 18 & 0 & 0 & 0 & 18 \\
\hline 221 & 2652 & 60 & KNTD RJCJ & 5.36534 & 23.3 & 0 & 0 & 0 & 23.3 \\
\hline 221 & 2654 & 85 & KHIF $\sim$ RJCC & 22.89513 & 0 & 0 & 41.4 & 0 & 41.4 \\
\hline 221 & 2655 & 62 & KNUQ RJFK & 25.80903 & 18 & 0 & 0 & 0 & 18 \\
\hline 221 & 2653 & 62 & KNUQ RJFK & 29.03074 & 18 & 0 & 0 & 0 & 18 \\
\hline 227 & 2724 & 62 & KNUQ RJFK & 24.8525 & 18 & 0 & 0 & 18 & 21.6 \\
\hline 228 & 2736 & 62 & KNUQ RJFK & 25.88379 & 18 & 0 & 0 & 71 & 32.2 \\
\hline 233 & 2796 & 57 & KNKT $\sim$ RJAA & 5.53619 & 35.53 & 0 & 0 & 0 & 35.53 \\
\hline 233 & 2797 & 55 & KSSC $\sim \mathrm{OMAA}$ & 12.30616 & 50.42 & 0 & 0 & 0 & 50.42 \\
\hline 233 & 2798 & 85 & KHIF $\sim$ RJCC & 22.14133 & 0 & 0 & 41.4 & 0 & 41.4 \\
\hline 233 & 2799 & 62 & KNUQ $\sim$ RJFK & 25.59297 & 18 & 0 & 0 & 0 & 18 \\
\hline 234 & 2809 & 55 & $\mathrm{KSSC} \sim \mathrm{OMAA}$ & 12.61152 & 61.5 & 0 & 0 & 0 & 61.5 \\
\hline 234 & 2810 & 85 & $\mathrm{KHIF} \sim \mathrm{RJCC}$ & 24.15452 & 0 & 0 & 41.4 & 0 & 41.4 \\
\hline 234 & 2811 & 62 & KNUQ RJFK & 27.1149 & 18 & 0 & 0 & 0 & 18 \\
\hline 234 & 2808 & 62 & KNUQ RJFK & 29.59947 & 18 & 0 & 0 & 0 & 18 \\
\hline 235 & 2820 & 57 & KNKT $\sim$ RJAA & 4.95765 & 41.3 & 0 & 0 & 0 & 41.3 \\
\hline 235 & 2821 & 55 & KSSC $\sim \mathrm{OMAA}$ & 12.46394 & 50.92 & 0 & 0 & 0 & 50.92 \\
\hline 235 & 2822 & 85 & KHIF $\sim$ RJCC & 21.54734 & 0 & 0 & 41.4 & 0 & 41.4 \\
\hline 235 & 2823 & 59 & KNLC $\sim$ RJCC & 25.4087 & 23.9 & 0 & 0 & 8 & 25.5 \\
\hline 236 & 2832 & 57 & KNKT $\sim$ RJAA & 5.50964 & 41.3 & 0 & 0 & 0 & 41.3 \\
\hline 236 & 2834 & 85 & KHIF $\sim$ RJCC & 21.29632 & 0 & 0 & 41.4 & 0 & 41.4 \\
\hline 236 & 2833 & 62 & KNUQ RJFK & 26.2399 & 18 & 0 & 0 & 0 & 18 \\
\hline 236 & 2835 & 94 & KBLV RJTT & 32.34795 & 9 & 0 & 0 & 0 & 9 \\
\hline 237 & 2844 & 57 & KNKT $\sim$ RJAA & 5.23108 & 41.3 & 0 & 0 & 0 & 41.3 \\
\hline 237 & 2845 & 52 & KSLC $\sim$ LTAG & 13.31986 & 0 & 33.6 & 0 & 0 & 33.6 \\
\hline 237 & 2846 & 85 & KHIF $\sim$ RJCC & 23.0045 & 0 & 0 & 41.4 & 0 & 41.4 \\
\hline 237 & 2847 & 74 & KORD RJFF & 26.09286 & 51.18 & 0 & 0 & 0 & 51.18 \\
\hline 238 & 2856 & 57 & KNKT $\sim$ RJAA & 5.53619 & 35.53 & 0 & 0 & 0 & 35.53 \\
\hline 238 & 2857 & 50 & KSKA LTAG & 12.73017 & 0 & 6.7 & 0 & 7 & 8.1 \\
\hline 238 & 2858 & 85 & KHIF $\sim$ RJCC & 23.21321 & 0 & 0 & 41.4 & 0 & 41.4 \\
\hline 238 & 2859 & 74 & KORD RJFF & 25.93661 & 16.02 & 0 & 0 & 0 & 16.02 \\
\hline 239 & 2868 & 57 & KNKT $\sim$ RJAA & 5.11377 & 13.63 & 0 & 0 & 0 & 13.63 \\
\hline 239 & 2869 & 75 & KPAM RJFK & 16.93717 & 0.5 & 11.2 & 0 & 0 & 11.7 \\
\hline 239 & 2870 & 85 & KHIF $\sim$ RJCC & 24.08889 & 0 & 0 & 41.4 & 0 & 41.4 \\
\hline 239 & 2871 & 91 & KNZJ $\sim$ RJOI & 27.25913 & 0 & 4.5 & 0 & 0 & 4.5 \\
\hline 240 & 2880 & 22 & KFSM LTAG & 7.23905 & 16.5 & 36.71 & 0 & 0 & 53.21 \\
\hline 240 & 2881 & 53 & KSPI LTAG & 17.97592 & 0 & 33.6 & 0 & 0 & 33.6 \\
\hline 240 & 2882 & 85 & KHIF $\sim$ RJCC & 22.67025 & 0 & 0 & 41.4 & 0 & 41.4 \\
\hline 241 & 2892 & 22 & KFSM LTAG & 6.97938 & 0 & 61.89 & 0 & 3 & 62.49 \\
\hline 241 & 2893 & 84 & KRCA RJBB & 15.00323 & 34.5 & 16.9 & 0 & 0 & 51.4 \\
\hline 241 & 2894 & 85 & $\mathrm{KHIF} \sim \mathrm{RJCC}$ & 21.35465 & 0 & 0 & 41.4 & 0 & 41.4 \\
\hline 242 & 2904 & 17 & KFMH LTAG & 8.66709 & 30.2 & 0 & 0 & 0 & 30.2 \\
\hline 242 & 2905 & 84 & KRCA RJBB & 14.847 & 10.9 & 17.7 & 0 & 0 & 28.6 \\
\hline 242 & 2906 & 85 & KHIF $\sim$ RJCC & 22.89513 & 0 & 0 & 41.4 & 0 & 41.4 \\
\hline 250 & 3000 & 15 & KFHU LTAG & 5.69164 & 9 & 0 & 0 & 0 & 9 \\
\hline 250 & 3001 & 67 & KNZJ RJTA & 15.70454 & 0 & 14.5 & 0 & 0 & 14.5 \\
\hline
\end{tabular}




\begin{tabular}{|c|c|c|c|c|c|c|c|c|c|}
\hline \multirow[b]{2}{*}{ Tail\# } & \multirow[b]{2}{*}{ Msn\# } & \multirow[b]{2}{*}{ REQT\# } & \multirow[b]{2}{*}{ APOE $\sim$ APOD } & \multirow[b]{2}{*}{ ARR APOD } & \multicolumn{5}{|c|}{-------DELIVERED QUANTITIES-------- } \\
\hline & & & & & OUT & OVER & BULK & PAX & TOTAL \\
\hline 250 & 3002 & 85 & KHIF $\sim$ RJCC & 20.95076 & 0 & 0 & 41.4 & 0 & 41.4 \\
\hline 251 & 3012 & 14 & KFFO LTAG & 6.46584 & 0 & 12.5 & 0 & 0 & 12.5 \\
\hline 251 & 3013 & 61 & KNTU RJFF & 16.28466 & 6.5 & 6.7 & 0 & 0 & 13.2 \\
\hline 251 & 3014 & 85 & KHIF RJCC & 21.25168 & 0 & 0 & 41.4 & 0 & 41.4 \\
\hline 252 & 3024 & 12 & KEND OMAA & 5.07023 & 0 & 4.5 & 0 & 0 & 4.5 \\
\hline 252 & 3025 & 68 & KNZW RJTT & 17.24112 & 50.5 & 0 & 0 & 0 & 50.5 \\
\hline 252 & 3026 & 85 & KHIF RJCC & 20.96952 & 0 & 0 & 41.4 & 0 & 41.4 \\
\hline 253 & 3036 & 30 & KGTB LTBA & 5.81859 & 0 & 8.8 & 0 & 12 & 11.2 \\
\hline 253 & 3037 & 68 & KNZW RJTT & 16.83531 & 39.8 & 0 & 1.2 & 0 & 41 \\
\hline 253 & 3038 & 85 & KHIF $\sim$ RJCC & 20.79713 & 0 & 0 & 41.4 & 0 & 41.4 \\
\hline 254 & 3048 & 18 & KFOE LTAC & 5.90084 & 6.7 & 50.84 & 0 & 0 & 57.54 \\
\hline 254 & 3049 & 55 & $\mathrm{KSSC} \sim \mathrm{OMAA}$ & 12.00451 & 35.96 & 0 & 0 & 0 & 35.96 \\
\hline 254 & 3050 & 85 & KHIF $\sim$ RJCC & 22.03399 & 0 & 0 & 41.4 & 0 & 41.4 \\
\hline 255 & 3060 & 18 & KFOE LTAC & 6.04901 & 0 & 47.9 & 0 & 0 & 47.9 \\
\hline 255 & 3061 & 83 & KPSM RJAA & 18.11236 & 26.2 & 0 & 0 & 0 & 26.2 \\
\hline 255 & 3062 & 85 & KHIF RJCC & 22.56212 & 0 & 0 & 41.4 & 0 & 41.4 \\
\hline 256 & 3072 & 18 & KFOE LTAC & 6.15592 & 0 & 45.46 & 0 & 0 & 45.46 \\
\hline 256 & 3073 & 83 & KPSM RJAA & 18.33849 & 26.2 & 0 & 0 & 0 & 26.2 \\
\hline 256 & 3074 & 85 & KHIF RJCC & 23.63187 & 0 & 0 & 41.4 & 0 & 41.4 \\
\hline 257 & 3084 & 31 & KBLV LTBF & 5.82181 & 5.6 & 6.7 & 0 & 1 & 12.5 \\
\hline 257 & 3085 & 83 & KPSM RJAA & 17.94472 & 26.2 & 0 & 0 & 0 & 26.2 \\
\hline 257 & 3086 & 85 & KHIF RJCC & 22.50252 & 0 & 0 & 41.4 & 0 & 41.4 \\
\hline 258 & 3096 & 37 & KNHK LTFJ & 7.79938 & 23.3 & 0 & 0 & 0 & 23.3 \\
\hline 258 & 3097 & 83 & KPSM RJAA & 17.83067 & 26.2 & 0 & 0 & 0 & 26.2 \\
\hline 258 & 3098 & 85 & KHIF $\sim$ RJCC & 20.93426 & 0 & 0 & 41.4 & 0 & 41.4 \\
\hline 259 & 3108 & 40 & KNGU LTAF & 8.5344 & 0 & 47.9 & 0 & 0 & 47.9 \\
\hline 259 & 3109 & 83 & KPSM RJAA & 18.34848 & 26.2 & 0 & 0 & 0 & 26.2 \\
\hline 259 & 3110 & 85 & KHIF $\sim$ RJCC & 22.38494 & 0 & 0 & 41.4 & 0 & 41.4 \\
\hline 260 & 3120 & 40 & KNGU LTAF & 8.711 & 0 & 47.9 & 0 & 0 & 47.9 \\
\hline 260 & 3121 & 83 & KPSM RJAA & 18.11236 & 26.2 & 0 & 0 & 0 & 26.2 \\
\hline 260 & 3122 & 85 & KHIF $\sim$ RJCC & 21.25168 & 0 & 0 & 41.4 & 0 & 41.4 \\
\hline 261 & 3132 & 40 & KNGU LTAF & 8.89561 & 0 & 14.6 & 0 & 0 & 14.6 \\
\hline 261 & 3133 & 83 & KPSM RJAA & 18.41141 & 26.2 & 0 & 0 & 0 & 26.2 \\
\hline 261 & 3134 & 85 & KHIF RJCC & 23.07921 & 0 & 0 & 41.4 & 0 & 41.4 \\
\hline 262 & 3144 & 39 & KNGP $\sim$ LTAC & 8.22629 & 38.1 & 0 & 0 & 0 & 38.1 \\
\hline 262 & 3145 & 83 & KPSM RJAA & 17.9447 & 12.3 & 0 & 0 & 0 & 12.3 \\
\hline 262 & 3146 & 85 & KHIF RJCC & 20.95076 & 0 & 0 & 41.4 & 0 & 41.4 \\
\hline 263 & 3156 & 39 & KNGP LTAC & 7.93506 & 38.1 & 0 & 0 & 0 & 38.1 \\
\hline 263 & 3157 & 81 & KPIT RJTT & 19.04081 & 9 & 0 & 41.4 & 0 & 50.4 \\
\hline 263 & 3158 & 85 & KHIF $\sim$ RJCC & 23.41099 & 0 & 0 & 41.4 & 0 & 41.4 \\
\hline 264 & 3168 & 39 & KNGP LTAC & 8.22913 & 38.1 & 0 & 0 & 0 & 38.1 \\
\hline 264 & 3169 & 81 & KPIT $\sim$ RJTT & 19.88065 & 0 & 0 & 41.4 & 0 & 41.4 \\
\hline 264 & 3170 & 85 & KHIF $\sim$ RJCC & 23.81696 & 0 & 0 & 41.4 & 0 & 41.4 \\
\hline 265 & 3180 & 39 & KNGP $\sim$ LTAC & 8.07993 & 38.1 & 0 & 0 & 0 & 38.1 \\
\hline 265 & 3181 & 81 & KPIT $\sim$ RJTT & 19.20842 & 0 & 0 & 41.4 & 0 & 41.4 \\
\hline 265 & 3182 & 85 & KHIF $\sim$ RJCC & 23.22875 & 0 & 0 & 17.54 & 0 & 17.54 \\
\hline 266 & 3192 & 39 & KNGP LTAC & 7.97125 & 38.1 & 0 & 0 & 0 & 38.1 \\
\hline 266 & 3193 & 81 & KPIT $\sim$ RJTT & 19.09429 & 0 & 0 & 41.4 & 0 & 41.4 \\
\hline 266 & 3194 & 85 & KHIF $\sim$ RJCC & 24.15292 & 0 & 0 & 41.4 & 0 & 41.4 \\
\hline 267 & 3204 & 39 & KNGP LTAC & 8.81204 & 13.5 & 0 & 0 & 0 & 13.5 \\
\hline 267 & 3205 & 81 & KPIT $\sim$ RJTT & 19.56488 & 0 & 0 & 2.4 & 0 & 2.4 \\
\hline 267 & 3206 & 85 & KHIF $\sim$ RJCC & 23.48556 & 0 & 0 & 41.4 & 0 & 41.4 \\
\hline 268 & 3216 & 38 & KNFW LTAG & 10.2668 & 6.5 & 6.7 & 0 & 0 & 13.2 \\
\hline 268 & 3217 & 88 & KNXX RJFK & 19.42022 & 0.5 & 11.2 & 34.57 & 0 & 46.27 \\
\hline 268 & 3218 & 85 & KHIF RJCC & 22.90882 & 0 & 0 & 41.4 & 0 & 41.4 \\
\hline 269 & 3228 & 24 & KFWH LTAG & 9.79769 & 22.3 & 0 & 0 & 0 & 22.3 \\
\hline 269 & 3229 & 88 & KNXX RJFK & 20.15135 & 0 & 0 & 41.4 & 0 & 41.4 \\
\hline 269 & 3230 & 85 & KHIF $\sim$ RJCC & 24.17168 & 0 & 0 & 41.4 & 0 & 41.4 \\
\hline
\end{tabular}




\begin{tabular}{|c|c|c|c|c|c|c|c|c|c|}
\hline & & & & & -------D & LIVERED & QUANTIT & ES----- & \\
\hline Tail\# & Msn\# & REQT\# & APOE $\sim$ APOD & ARR APOD & OUT & OVER & BULK & PAX & TOTAL \\
\hline 270 & 3240 & 23 & KFWA LTAG & 9.76296 & 0 & 0 & 22.1 & 0 & 22.1 \\
\hline 270 & 3241 & 88 & KNXX RJFK & 18.8705 & 0 & 0 & 41.4 & 0 & 41.4 \\
\hline 270 & 3242 & 85 & KHIF $\sim$ RJCC & 21.12867 & 0 & 0 & 41.4 & 0 & 41.4 \\
\hline 271 & 3252 & 32 & KBLV LTBG & 7.85176 & 0 & 31.4 & 0 & 0 & 31.4 \\
\hline 271 & 3253 & 88 & KNXX RJFK & 19.36871 & 0 & 0 & 41.4 & 0 & 41.4 \\
\hline 271 & 3254 & 85 & KHIF $\sim$ RJCC & 21.94582 & 0 & 0 & 41.4 & 0 & 41.4 \\
\hline 272 & 3264 & 32 & KBLV $\sim$ LTBG & 7.99994 & 0 & 31.4 & 0 & 0 & 31.4 \\
\hline 272 & 3265 & 88 & KNXX RJFK & 19.18291 & 0 & 0 & 41.4 & 0 & 41.4 \\
\hline 272 & 3266 & 85 & KHIF $\sim \mathrm{RJCC}$ & 22.65549 & 0 & 0 & 41.4 & 0 & 41.4 \\
\hline 273 & 3276 & 32 & KBLV LTBG & 7.81916 & 0 & 13.1 & 0 & 0 & 13.1 \\
\hline 273 & 3277 & 88 & KNXX $\sim$ RJFK & 19.31084 & 0 & 0 & 41.4 & 0 & 41.4 \\
\hline 273 & 3278 & 85 & KHIF RJCC & 23.2926 & 0 & 0 & 41.4 & 0 & 41.4 \\
\hline 274 & 3288 & 42 & KNHK $\sim$ ENBO & 9.72644 & 32 & 0 & 0 & 0 & 32 \\
\hline 274 & 3289 & 88 & KNXX RJFK & 20.15135 & 0 & 0 & 41.4 & 0 & 41.4 \\
\hline 274 & 3290 & 85 & KHIF $\sim$ RJCC & 23.53626 & 0 & 0 & 41.4 & 0 & 41.4 \\
\hline 275 & 3301 & 88 & KNXX RJFK & 19.7455 & 0 & 0 & 41.4 & 0 & 41.4 \\
\hline 275 & 3302 & 70 & KOAK $\sim$ RJAA & 22.03675 & 18 & 0 & 0 & 0 & 18 \\
\hline 276 & 3312 & 36 & KNGZ LTCJ & 10.06368 & 23.9 & 0 & 0 & 8 & 25.5 \\
\hline 276 & 3313 & 88 & KNXX RJFK & 19.47798 & 0 & 0 & 21.13 & 0 & 21.13 \\
\hline 276 & 3314 & 56 & KSTJ OMAD & 23.04018 & 0 & 14.2 & 0 & 0 & 14.2 \\
\hline 277 & 3324 & 34 & KNGP $\sim$ LTBU & 11.07895 & 50.5 & 0 & 0 & 0 & 50.5 \\
\hline 277 & 3325 & 73 & KOQU RJCJ & 20.37114 & 0 & 6.6 & 0 & 0 & 6.6 \\
\hline 277 & 3326 & 80 & KPIA RJTA & 24.76563 & 0 & 12.5 & 0 & 0 & 12.5 \\
\hline 278 & 3336 & 34 & KNGP $\sim$ LTBU & 10.20635 & 50.5 & 0 & 0 & 0 & 50.5 \\
\hline 278 & 3337 & 72 & KOKC $\sim \mathrm{RJCC}$ & 20.95992 & 0 & 0 & 41.4 & 0 & 41.4 \\
\hline 278 & 3338 & 78 & KPHL RJOI & 26.45675 & 0 & 4.5 & 0 & 0 & 4.5 \\
\hline 279 & 3348 & 34 & KNGP $\sim$ LTBU & 10.16767 & 50.5 & 0 & 0 & 0 & 50.5 \\
\hline 279 & 3349 & 72 & KOKC $\sim$ RJCC & 21.56709 & 0 & 0 & 41.4 & 0 & 41.4 \\
\hline 279 & 3350 & 93 & KNZY RJTA & 24.89339 & 0 & 12.5 & 0 & 0 & 12.5 \\
\hline 280 & 3360 & 34 & KNGP $\sim$ LTBU & 10.64117 & 50.5 & 0 & 0 & 0 & 50.5 \\
\hline 280 & 3361 & 72 & KOKC $\sim \mathrm{RJCC}$ & 20.8379 & 0 & 0 & 41.4 & 0 & 41.4 \\
\hline 280 & 3362 & 87 & KNUW RJFF & 25.22579 & 26.2 & 0 & 0 & 0 & 26.2 \\
\hline 281 & 3372 & 34 & KNGP $\sim$ LTBU & 10.78935 & 50.5 & 0 & 0 & 0 & 50.5 \\
\hline 281 & 3373 & 72 & $\mathrm{KOKC} \sim \mathrm{RJCC}$ & 20.72385 & 0 & 0 & 41.4 & 0 & 41.4 \\
\hline 281 & 3374 & 87 & KNUW RJFF & 25.06704 & 26.2 & 0 & 0 & 0 & 26.2 \\
\hline 282 & 3384 & 34 & KNGP $\sim$ LTBU & 11.33484 & 50.5 & 0 & 0 & 0 & 50.5 \\
\hline 282 & 3385 & 72 & KOKC $\sim$ RJCC & 21.54731 & 0 & 0 & 41.4 & 0 & 41.4 \\
\hline 282 & 3386 & 87 & KNUW RJFF & 25.20457 & 14.8 & 0 & 0 & 0 & 14.8 \\
\hline 283 & 3396 & 34 & KNGP $\sim$ LTBU & 11.07895 & 50.5 & 0 & 0 & 0 & 50.5 \\
\hline 283 & 3398 & 86 & KBLV RJCJ & 14.1185 & 0 & 6.6 & 0 & 0 & 6.6 \\
\hline 283 & 3397 & 72 & KOKC $\sim$ RJCC & 20.95992 & 0 & 0 & 41.4 & 0 & 41.4 \\
\hline 284 & 3408 & 34 & KNGP $\sim$ LTBU & 11.22719 & 50.5 & 0 & 0 & 0 & 50.5 \\
\hline 284 & 3409 & 72 & KOKC $\sim \mathrm{RJCC}$ & 21.56709 & 0 & 0 & 41.4 & 0 & 41.4 \\
\hline 284 & 3410 & 62 & KNUQ RJFK & 25.71412 & 23.7 & 0 & 0 & 0 & 23.7 \\
\hline 285 & 3420 & 34 & KNGP $\sim$ LTBU & 10.89678 & 46.6 & 0 & 0 & 0 & 46.6 \\
\hline 285 & 3421 & 72 & KOKC $\sim \mathrm{RJCC}$ & 20.8379 & 0 & 0 & 41.4 & 0 & 41.4 \\
\hline 285 & 3422 & 62 & KNUQ $\sim$ RJFK & 25.01668 & 23.7 & 0 & 0 & 0 & 23.7 \\
\hline 286 & 3432 & 47 & KBNA OJ1X & 9.8768 & 18 & 0 & 0 & 0 & 18 \\
\hline 286 & 3434 & 65 & KNYL $\sim$ RJOI & 13.39867 & 16.5 & 0 & 0 & 0 & 16.5 \\
\hline 286 & 3433 & 72 & $\mathrm{KOKC} \sim \mathrm{RJCC}$ & 20.72385 & 0 & 0 & 41.4 & 0 & 41.4 \\
\hline 287 & 3446 & 57 & KNKT $\sim$ RJAA & 4.94426 & 42 & 0 & 0 & 0 & 42 \\
\hline 287 & 3444 & 43 & KBLV ENTC & 9.79835 & 0 & 10.5 & 8.9 & 0 & 19.4 \\
\hline 287 & 3445 & 72 & $\mathrm{KOKC} \sim \mathrm{RJCC}$ & 21.54731 & 0 & 0 & 41.4 & 0 & 41.4 \\
\hline 288 & 3456 & 45 & KWRI ETAR & 9.5546 & 26.2 & 0 & 0 & 0 & 26.2 \\
\hline 288 & 3457 & 72 & $\mathrm{KOKC} \sim \mathrm{RJCC}$ & 20.95992 & 0 & 0 & 41.4 & 0 & 41.4 \\
\hline 289 & 3468 & 45 & KWRI ETAR & 9.43654 & 26.2 & 0 & 1.2 & 0 & 27.4 \\
\hline 289 & 3470 & 66 & KNZC $\sim$ RJSM & 20.80529 & 0 & 10.5 & 0 & 0 & 10.5 \\
\hline 289 & 3469 & 72 & $\mathrm{KOKC} \sim \mathrm{RJCC}$ & 23.83555 & 0 & 0 & 32.1 & 0 & 32.1 \\
\hline
\end{tabular}




\begin{tabular}{|c|c|c|c|c|c|c|c|c|c|}
\hline & & & & & \multicolumn{5}{|c|}{-----DELIVERED QUANTITIES------- } \\
\hline Tail\# & Msn\# & REQT\# & APOE $\sim$ APOD & ARR APOD & OUT & OVER & BULK & PAX & TOTAL \\
\hline 290 & 3480 & 62 & KNUQ RJFK & 25.60001 & 23.7 & 0 & 0 & 0 & 23.7 \\
\hline 290 & 3482 & 62 & KNUQ RJFK & 27.71175 & 8.9 & 0 & 0 & 0 & 8.9 \\
\hline
\end{tabular}

Table A.3 Scenario 3 Mission Summary

\section{A.4 SCENARIO 4 Best Solution Mission Summary}

\begin{tabular}{|c|c|c|c|c|c|c|c|c|c|}
\hline & & & & & --------D & LIVERED & QUANTIT & ES------- & \\
\hline Tail\# & Msn\# & REQT\# & APOE APOD & ARR APOD & OUT & OVER & BULK & PAX & TOTAL \\
\hline 0 & 0 & 4 & KDOV OKBK & 0.92256 & 0 & 0 & 0 & 161 & 32.2 \\
\hline 0 & 0 & 5 & KDOV OKBK & 0.92256 & 0 & 0 & 0 & 5 & 1 \\
\hline 0 & 1 & 19 & KDOV LTAG & 10.82988 & 0 & 0 & 0 & 305 & 61 \\
\hline 0 & 2 & 37 & KDMA RJBB & 25.39351 & 0 & 0 & 0 & 335 & 67 \\
\hline 0 & 2 & 49 & KDMA RJBB & 25.39351 & 0 & 0 & 0 & 24 & 4.8 \\
\hline 1 & 12 & 0 & KBLV LTAG & 1.15861 & 0 & 0 & 0 & 300 & 60 \\
\hline 1 & 14 & 37 & KDMA $\sim \mathrm{RJBB}$ & 24.63572 & 0 & 0 & 0 & 310 & 62 \\
\hline 2 & 24 & 3 & KDMA $\sim \mathrm{OKBK}$ & 1.92282 & 0 & 0 & 0 & 335 & 67 \\
\hline 2 & 25 & 29 & KDOV LTAG & 12.84874 & 0 & 0 & 0 & 335 & 67 \\
\hline 2 & 26 & 37 & KDMA $\sim$ RJBB & 24.62856 & 0 & 0 & 0 & 280 & 56 \\
\hline 3 & 36 & 3 & KDMA OKBK & 2.21371 & 0 & 0 & 0 & 335 & 67 \\
\hline 3 & 37 & 29 & KDOV LTAG & 12.24656 & 0 & 0 & 0 & 335 & 67 \\
\hline 3 & 38 & 54 & KBLV RJBB & 25.62282 & 0 & 0 & 0 & 310 & 62 \\
\hline 4 & 48 & 10 & KDMA LTAG & 5.41254 & 0 & 0 & 0 & 146 & 29.2 \\
\hline 4 & 49 & 29 & KDOV LTAG & 11.81598 & 0 & 0 & 0 & 335 & 67 \\
\hline 4 & 50 & 54 & KBLV RJBB & 25.25137 & 0 & 0 & 0 & 280 & 56 \\
\hline 5 & 60 & 3 & KDMA $\sim \mathrm{OKBK}$ & 1.92282 & 0 & 0 & 0 & 335 & 67 \\
\hline 5 & 61 & 29 & KDOV LTAG & 12.10064 & 0 & 0 & 0 & 335 & 67 \\
\hline 5 & 62 & 54 & KBLV RJBB & 25.01248 & 0 & 0 & 0 & 335 & 67 \\
\hline 6 & 72 & 3 & KDMA $\sim \mathrm{OKBK}$ & 2.21371 & 0 & 0 & 0 & 335 & 67 \\
\hline 6 & 73 & 29 & KDOV LTAG & 12.11955 & 0 & 0 & 0 & 264 & 52.8 \\
\hline 6 & 74 & 54 & KBLV RJBB & 26.31969 & 0 & 0 & 0 & 305 & 61 \\
\hline 7 & 84 & 25 & KDMA ETAR & 1.79609 & 0 & 0 & 0 & 120 & 24 \\
\hline 8 & 96 & 9 & KBLV LTAG & 4.72281 & 0 & 0 & 0 & 28 & 5.6 \\
\hline 8 & 96 & 11 & KBLV LTAG & 4.72281 & 0 & 0 & 0 & 3 & 0.6 \\
\hline 9 & 108 & 7 & KDSM LTAG & 3.18476 & 0 & 0 & 0 & 335 & 67 \\
\hline 9 & 109 & 30 & KBLV LTAG & 11.86169 & 0 & 0 & 0 & 118 & 23.6 \\
\hline 10 & 120 & 7 & KDSM LTAG & 1.87918 & 0 & 0 & 0 & 335 & 67 \\
\hline 21 & 253 & 42 & KDOV $\sim \mathrm{RJBB}$ & 14.78855 & 0 & 0 & 0 & 195 & 39 \\
\hline 22 & 264 & 7 & KDSM LTAG & 2.89294 & 0 & 0 & 0 & 195 & 39 \\
\hline 22 & 265 & 42 & KDOV $\sim \mathrm{RJBB}$ & 14.90332 & 0 & 0 & 0 & 195 & 39 \\
\hline 23 & 276 & 7 & KDSM LTAG & 2.15149 & 0 & 0 & 0 & 195 & 39 \\
\hline 23 & 277 & 42 & KDOV RJBB & 15.51355 & 0 & 0 & 0 & 195 & 39 \\
\hline 24 & 288 & 7 & KDSM LTAG & 1.70674 & 0 & 0 & 0 & 195 & 39 \\
\hline 24 & 289 & 42 & KDOV $\sim \mathrm{RJBB}$ & 15.31665 & 0 & 0 & 0 & 93 & 18.6 \\
\hline 24 & 289 & 53 & KDOV $\sim \mathrm{RJBB}$ & 15.31665 & 0 & 0 & 0 & 101 & 20.2 \\
\hline 25 & 300 & 7 & KDSM LTAG & 2.45526 & 0 & 0 & 0 & 105 & 21 \\
\hline 25 & 301 & 53 & KDOV $\sim \mathrm{RJBB}$ & 14.70644 & 0 & 0 & 0 & 195 & 39 \\
\hline 26 & 313 & 53 & KDOV RJBB & 14.81736 & 0 & 0 & 0 & 38 & 7.6 \\
\hline 26 & 313 & 36 & KDOV RJBB & 14.81736 & 0 & 0 & 0 & 4 & 0.8 \\
\hline 26 & 313 & 50 & KDOV $\sim$ RJBB & 14.81736 & 0 & 0 & 0 & 8 & 1.6 \\
\hline 27 & 324 & 35 & KDOV RJBB & 5.51355 & 0 & 0 & 0 & 195 & 39 \\
\hline 27 & 325 & 45 & KDMA RJBB & 15.69815 & 0 & 0 & 0 & 120 & 24 \\
\hline 28 & 336 & 35 & KDOV RJBB & 5.04355 & 0 & 0 & 0 & 59 & 11.8 \\
\hline 28 & 337 & 39 & KBLV RJBB & 15.65626 & 0 & 0 & 0 & 123 & 24.6 \\
\hline 28 & 337 & 57 & KBLV RJBB & 15.65626 & 0 & 0 & 0 & 46 & 9.2 \\
\hline
\end{tabular}




\begin{tabular}{|c|c|c|c|c|c|c|c|c|c|}
\hline \multirow[b]{2}{*}{ Tail\# } & \multirow[b]{2}{*}{ Msn\# } & \multirow[b]{2}{*}{ REQT\# } & \multirow[b]{2}{*}{ APOE $\sim$ APOD } & \multirow[b]{2}{*}{ ARR APOD } & \multicolumn{5}{|c|}{--------DELIVERED QUANTITIES--------- } \\
\hline & & & & & OUT & OVER & BULK & PAX & TOTAL \\
\hline 29 & 348 & 10 & KDMA LTAG & 4.93866 & 0 & 0 & 0 & 195 & 39 \\
\hline 29 & 349 & 34 & KDMA OKBK & 22.90827 & 0 & 0 & 0 & 133 & 26.6 \\
\hline 29 & 349 & 32 & KDMA OKBK & 22.90827 & 0 & 0 & 0 & 12 & 2.4 \\
\hline 30 & 360 & 3 & KDMA $\sim \mathrm{OKBK}$ & 2.34504 & 0 & 0 & 0 & 195 & 39 \\
\hline 30 & 361 & 51 & KBLV RJBB & 23.84507 & 0 & 0 & 0 & 123 & 24.6 \\
\hline 31 & 373 & 58 & KDOV RJBB & 25.95178 & 0 & 0 & 0 & 123 & 24.6 \\
\hline 32 & 384 & 38 & KDMA RJBB & 5.37763 & 0 & 0 & 0 & 184 & 36.8 \\
\hline 34 & 408 & 15 & KBLV LTAG & 5.70322 & 0 & 0 & 0 & 195 & 39 \\
\hline 34 & 409 & 37 & KDMA RJBB & 25.02637 & 0 & 0 & 0 & 195 & 39 \\
\hline 35 & 420 & 15 & KBLV LTAG & 6.42514 & 0 & 0 & 0 & 195 & 39 \\
\hline 35 & 421 & 37 & KDMA RJBB & 25.42368 & 0 & 0 & 0 & 195 & 39 \\
\hline 36 & 432 & 15 & KBLV LTAG & 5.71532 & 0 & 0 & 0 & 52 & 10.4 \\
\hline 36 & 432 & 16 & KBLV LTAG & 5.71532 & 0 & 0 & 0 & 41 & 8.2 \\
\hline 36 & 433 & 37 & KDMA RJBB & 25.52348 & 0 & 0 & 0 & 94 & 18.8 \\
\hline 36 & 433 & 49 & KDMA RJBB & 25.52348 & 0 & 0 & 0 & 100 & 20 \\
\hline 37 & 445 & 37 & KDMA $\sim$ RJBB & 25.73677 & 0 & 0 & 0 & 195 & 39 \\
\hline 38 & 456 & 22 & KDMA ETAR & 7.59349 & 0 & 0 & 0 & 195 & 39 \\
\hline 38 & 457 & 49 & KDMA RJBB & 25.18989 & 0 & 0 & 0 & 195 & 39 \\
\hline 39 & 468 & 22 & KDMA ETAR & 7.59349 & 0 & 0 & 0 & 195 & 39 \\
\hline 39 & 469 & 49 & KDMA $\sim$ RJBB & 24.79842 & 0 & 0 & 0 & 195 & 39 \\
\hline 40 & 481 & 49 & KDMA RJBB & 24.75477 & 0 & 0 & 0 & 195 & 39 \\
\hline 41 & 492 & 12 & KBLV LTAG & 7.69597 & 0 & 0 & 0 & 195 & 39 \\
\hline 41 & 493 & 49 & KDMA RJBB & 25.09929 & 0 & 0 & 0 & 195 & 39 \\
\hline 42 & 504 & 12 & KBLV LTAG & 8.00716 & 0 & 0 & 0 & 195 & 39 \\
\hline 42 & 505 & 49 & KDMA $\sim \mathrm{RJBB}$ & 25.13202 & 0 & 0 & 0 & 195 & 39 \\
\hline 43 & 516 & 12 & KBLV LTAG & 8.86279 & 0 & 0 & 0 & 195 & 39 \\
\hline 44 & 528 & 12 & KBLV LTAG & 7.99471 & 0 & 0 & 0 & 58 & 11.6 \\
\hline 44 & 528 & 18 & KBLV LTAG & 7.99471 & 0 & 0 & 0 & 2 & 0.4 \\
\hline 44 & 529 & 49 & KDMA RJBB & 26.29905 & 0 & 0 & 0 & 131 & 26.2 \\
\hline 44 & 529 & 55 & KDMA RJBB & 26.29905 & 0 & 0 & 0 & 18 & 3.6 \\
\hline 65 & 780 & 4 & KDOV OKBK & 1.06045 & 0 & 0 & 87.4 & 0 & 87.4 \\
\hline 65 & 781 & 12 & KBLV LTAG & 8.48643 & 0 & 0 & 72.2 & 0 & 72.2 \\
\hline 65 & 782 & 36 & KDOV RJBB & 12.83923 & 0 & 0 & 69.51 & 0 & 69.51 \\
\hline 66 & 792 & 4 & KDOV OKBK & 0.91541 & 0 & 0 & 87.4 & 0 & 87.4 \\
\hline 66 & 793 & 12 & KBLV LTAG & 9.38038 & 0 & 0 & 72.2 & 0 & 72.2 \\
\hline 66 & 794 & 36 & KDOV $\sim \mathrm{RJBB}$ & 12.88397 & 0 & 0 & 20.89 & 0 & 20.89 \\
\hline 67 & 804 & 4 & KDOV OKBK & 1.06047 & 0 & 0 & 51.55 & 0 & 51.55 \\
\hline 67 & 804 & 6 & KDOV OKBK & 1.06047 & 0 & 0 & 45.95 & 0 & 45.95 \\
\hline 67 & 805 & 12 & KBLV LTAG & 8.48653 & 0 & 0 & 72.2 & 0 & 72.2 \\
\hline 67 & 806 & 53 & KDOV RJBB & 15.21214 & 0 & 0 & 56.9 & 0 & 56.9 \\
\hline 68 & 817 & 12 & KBLV LTAG & 8.78509 & 0 & 0 & 72.2 & 0 & 72.2 \\
\hline 68 & 816 & 27 & KDOV OKBK & 12.30325 & 0 & 0 & 72.2 & 0 & 72.2 \\
\hline 68 & 818 & 37 & KDMA RJBB & 25.58073 & 0 & 0 & 87.4 & 0 & 87.4 \\
\hline 69 & 829 & 24 & KDOV ETAR & 9.50815 & 0 & 0 & 8.9 & 0 & 8.9 \\
\hline 69 & 829 & 26 & KDOV ETAR & 9.50815 & 0 & 0 & 1.2 & 0 & 1.2 \\
\hline 69 & 828 & 27 & KDOV OKBK & 11.86496 & 0 & 0 & 72.2 & 0 & 72.2 \\
\hline 69 & 830 & 31 & KBLV LTAG & 15.1948 & 0 & 0 & 87.4 & 0 & 87.4 \\
\hline 70 & 840 & 27 & KDOV OKBK & 12.49769 & 0 & 0 & 72.2 & 0 & 72.2 \\
\hline 70 & 841 & 29 & KDOV LTAG & 14.37095 & 0 & 0 & 87.4 & 0 & 87.4 \\
\hline 70 & 842 & 31 & KBLV LTAG & 16.18218 & 0 & 0 & 87.4 & 0 & 87.4 \\
\hline 71 & 852 & 27 & KDOV OKBK & 11.81635 & 0 & 0 & 72.2 & 0 & 72.2 \\
\hline 71 & 853 & 29 & KDOV LTAG & 13.68926 & 0 & 0 & 87.4 & 0 & 87.4 \\
\hline 71 & 854 & 31 & KBLV LTAG & 15.4816 & 0 & 0 & 72.1 & 0 & 72.1 \\
\hline 72 & 864 & 1 & KBLV LTAG & 1.21873 & 0 & 0 & 23.5 & 0 & 23.5 \\
\hline 72 & 866 & 29 & KDOV $\sim \mathrm{LTAG}$ & 12.14215 & 0 & 0 & 87.4 & 0 & 87.4 \\
\hline 72 & 865 & 29 & KDOV LTAG & 13.82912 & 0 & 0 & 87.4 & 0 & 87.4 \\
\hline 73 & 876 & 20 & KDOV $\sim \mathrm{LTAG}$ & 9.16304 & 0 & 0 & 51.2 & 0 & 51.2 \\
\hline 73 & 877 & 29 & KDOV LTAG & 12.45276 & 0 & 0 & 87.4 & 0 & 87.4 \\
\hline
\end{tabular}




\begin{tabular}{|c|c|c|c|c|c|c|c|c|c|}
\hline & & & & & \multicolumn{5}{|c|}{-------DELIVERED QUANTITIES-------- } \\
\hline Tail\# & Msn\# & REQT\# & APOE APOD & ARR APOD & OUT & OVER & BULK & PAX & TOTAL \\
\hline 73 & 878 & 46 & KDOV $\sim \mathrm{RJBB}$ & 17.12994 & 0 & 0 & 6.2 & 0 & 6.2 \\
\hline 74 & 889 & 29 & KDOV LTAG & 13.34532 & 0 & 0 & 18.4 & 0 & 18.4 \\
\hline 74 & 888 & 39 & KBLV RJBB & 15.95788 & 0 & 0 & 5.6 & 0 & 5.6 \\
\hline 74 & 888 & 57 & KBLV RJBB & 15.95788 & 0 & 0 & 56 & 0 & 56 \\
\hline 74 & 890 & 44 & KBLV $\sim$ RJBB & 21.07324 & 0 & 0 & 8.9 & 0 & 8.9 \\
\hline 75 & 900 & 3 & KDMA OKBK & 1.96362 & 0 & 0 & 87.4 & 0 & 87.4 \\
\hline 75 & 902 & 37 & KDMA RJBB & 24.85955 & 0 & 0 & 75.67 & 0 & 75.67 \\
\hline 76 & 913 & 6 & KDOV OKBK & 1.35212 & 0 & 0 & 87.4 & 0 & 87.4 \\
\hline 76 & 912 & 3 & KDMA OKBK & 3.64887 & 0 & 0 & 87.4 & 0 & 87.4 \\
\hline 76 & 914 & 37 & KDMA RJBB & 24.85239 & 0 & 0 & 69.51 & 0 & 69.51 \\
\hline 77 & 925 & 6 & KDOV OKBK & 1.40153 & 0 & 0 & 87.4 & 0 & 87.4 \\
\hline 77 & 924 & 3 & KDMA OKBK & 3.67396 & 0 & 0 & 87.4 & 0 & 87.4 \\
\hline 77 & 926 & 37 & KDMA RJBB & 25.41572 & 0 & 0 & 87.4 & 0 & 87.4 \\
\hline 78 & 936 & 3 & KDMA OKBK & 1.9636 & 0 & 0 & 78.63 & 0 & 78.63 \\
\hline 78 & 937 & 27 & KDOV OKBK & 12.25464 & 0 & 0 & 72.2 & 0 & 72.2 \\
\hline 78 & 938 & 30 & KBLV LTAG & 14.24431 & 0 & 0 & 83.74 & 0 & 83.74 \\
\hline 79 & 949 & 6 & KDOV OKBK & 1.20627 & 0 & 0 & 79.85 & 0 & 79.85 \\
\hline 79 & 948 & 9 & KBLV LTAG & 3.47633 & 0 & 0 & 45.5 & 0 & 45.5 \\
\hline 79 & 950 & 37 & KDMA RJBB & 26.16402 & 0 & 0 & 72.86 & 0 & 72.86 \\
\hline 80 & 960 & 10 & KDMA LTAG & 5.43526 & 0 & 0 & 10.2 & 0 & 10.2 \\
\hline 80 & 961 & 27 & KDOV OKBK & 11.99864 & 0 & 0 & 72.2 & 0 & 72.2 \\
\hline 81 & 972 & 38 & KDMA $\sim \mathrm{RJBB}$ & 5.50196 & 0 & 0 & 65.53 & 0 & 65.53 \\
\hline 81 & 973 & 27 & KDOV OKBK & 12.54631 & 0 & 0 & 72.2 & 0 & 72.2 \\
\hline 82 & 984 & 38 & KDMA RJBB & 4.67726 & 0 & 0 & 75.67 & 0 & 75.67 \\
\hline 82 & 985 & 27 & KDOV OKBK & 12.39968 & 0 & 0 & 72.2 & 0 & 72.2 \\
\hline 83 & 996 & 38 & KDMA RJBB & 4.96177 & 0 & 0 & 69.51 & 0 & 69.51 \\
\hline 83 & 997 & 27 & KDOV OKBK & 12.09665 & 0 & 0 & 72.2 & 0 & 72.2 \\
\hline 84 & 1008 & 38 & KDMA RJBB & 5.19685 & 0 & 0 & 31.45 & 0 & 31.45 \\
\hline 84 & 1009 & 27 & KDOV OKBK & 12.39968 & 0 & 0 & 72.2 & 0 & 72.2 \\
\hline 112 & 1344 & 2 & KDOV LTAG & 1.43289 & 0 & 18.1 & 0 & 0 & 18.1 \\
\hline 112 & 1346 & 8 & KDYS LTAG & 4.16798 & 37.2 & 0 & 0 & 0 & 37.2 \\
\hline 112 & 1347 & 40 & KDOV RJBB & 25.76336 & 18 & 0 & 0 & 0 & 18 \\
\hline 112 & 1345 & 40 & KDOV RJBB & 28.62361 & 18 & 0 & 0 & 0 & 18 \\
\hline 113 & 1356 & 4 & KDOV OKBK & 0.94073 & 13 & 6 & 53.65 & 72 & 87.05 \\
\hline 113 & 1358 & 41 & KBLV RJBB & 12.18793 & 0 & 62.17 & 0 & 0 & 62.17 \\
\hline 113 & 1357 & 46 & KDOV RJBB & 17.12625 & 50.2 & 0 & 0 & 0 & 50.2 \\
\hline 113 & 1359 & 40 & KDOV RJBB & 25.50717 & 18 & 0 & 0 & 0 & 18 \\
\hline 114 & 1368 & 0 & KBLV LTAG & 1.24154 & 0 & 33.5 & 0 & 0 & 33.5 \\
\hline 114 & 1368 & 1 & KBLV LTAG & 1.24154 & 23.78 & 0 & 0 & 0 & 23.78 \\
\hline 114 & 1370 & 3 & KDMA OKBK & 4.19003 & 0 & 0 & 73.17 & 41 & 81.37 \\
\hline 114 & 1369 & 12 & KBLV LTAG & 9.77556 & 0 & 0 & 65.27 & 0 & 65.27 \\
\hline 114 & 1371 & 40 & KDOV RJBB & 25.65415 & 18 & 0 & 0 & 67 & 31.4 \\
\hline 114 & 1371 & 47 & KDOV RJBB & 25.65415 & 0 & 0 & 0 & 221 & 44.2 \\
\hline 115 & 1380 & 1 & KBLV LTAG & 2.78575 & 9.42 & 0 & 0 & 0 & 9.42 \\
\hline 115 & 1380 & 9 & KBLV LTAG & 2.78575 & 0.5 & 11.2 & 0 & 0 & 11.7 \\
\hline 115 & 1381 & 19 & KDOV LTAG & 11.01632 & 55.9 & 0 & 0 & 0 & 55.9 \\
\hline 115 & 1382 & 41 & KBLV $\sim$ RJBB & 13.64869 & 0 & 69.05 & 0 & 0 & 69.05 \\
\hline 115 & 1383 & 40 & KDOV RJBB & 25.73385 & 18 & 0 & 0 & 0 & 18 \\
\hline 116 & 1392 & 25 & KDMA ETAR & 1.84848 & 0 & 14.5 & 0 & 0 & 14.5 \\
\hline 116 & 1394 & 41 & KBLV RJBB & 12.45053 & 0 & 55.54 & 0 & 0 & 55.54 \\
\hline 116 & 1393 & 41 & KBLV RJBB & 15.3434 & 0 & 64.04 & 0 & 0 & 64.04 \\
\hline 116 & 1395 & 40 & KDOV RJBB & 25.27197 & 18 & 0 & 0 & 0 & 18 \\
\hline 117 & 1405 & 23 & KDMA ETAR & 9.84855 & 32 & 0 & 0 & 0 & 32 \\
\hline 117 & 1405 & 22 & KDMA ETAR & 9.84855 & 0 & 0 & 0 & 8 & 1.6 \\
\hline 117 & 1406 & 53 & KDOV RJBB & 15.04274 & 34.5 & 16.9 & 0 & 0 & 51.4 \\
\hline 117 & 1407 & 40 & KDOV $\sim$ RJBB & 25.00637 & 18 & 0 & 0 & 0 & 18 \\
\hline 118 & 1416 & 26 & KDOV ETAR & 9.52755 & 34.5 & 0 & 0 & 0 & 34.5 \\
\hline 118 & 1417 & 43 & KDMA RJBB & 12.36064 & 16.5 & 0 & 0 & 0 & 16.5 \\
\hline
\end{tabular}




\begin{tabular}{|c|c|c|c|c|c|c|c|c|c|}
\hline & & & & & \multicolumn{5}{|c|}{--------DELIVERED QUANTITIES--------- } \\
\hline Tail\# & Msn\# & REQT\# & APOE $\sim$ APOD & ARR APOD & OUT & OVER & BULK & PAX & TOTAL \\
\hline 118 & 1419 & 43 & KDMA RJBB & 15.4322 & 15.5 & 0 & 0 & 0 & 15.5 \\
\hline 118 & 1418 & 53 & KDOV $\sim \mathrm{RJBB}$ & 18.52309 & 10.9 & 17.7 & 0 & 0 & 28.6 \\
\hline 119 & 1428 & 7 & KDSM LTAG & 3.49924 & 0 & 0 & 0 & 340 & 68 \\
\hline 119 & 1429 & 15 & KBLV LTAG & 6.23277 & 0 & 66.97 & 0 & 0 & 66.97 \\
\hline 119 & 1431 & 58 & KDOV RJBB & 25.96998 & 34 & 0 & 0 & 0 & 34 \\
\hline 120 & 1440 & 35 & KDOV RJBB & 5.25207 & 39.78 & 0 & 0 & 68 & 53.38 \\
\hline 120 & 1441 & 30 & KBLV LTAG & 12.61129 & 1.2 & 33.6 & 30.66 & 59 & 77.26 \\
\hline 120 & 1442 & 45 & KDMA $\sim$ RJBB & 16.36079 & 0 & 44.5 & 0 & 0 & 44.5 \\
\hline 121 & 1452 & 35 & KDOV $\sim \mathrm{RJBB}$ & 5.00644 & 41.3 & 0 & 0 & 0 & 41.3 \\
\hline 121 & 1453 & 19 & KDOV $\sim$ LTAG & 11.1286 & 41.2 & 0 & 0 & 0 & 41.2 \\
\hline 121 & 1454 & 39 & KBLV RJBB & 16.07014 & 6.5 & 6.7 & 0 & 0 & 13.2 \\
\hline 121 & 1454 & 57 & KBLV RJBB & 16.07014 & 0 & 20.9 & 0 & 0 & 20.9 \\
\hline 127 & 1524 & 37 & KDMA RJBB & 25.17754 & 0 & 0 & 62.56 & 0 & 62.56 \\
\hline 133 & 1596 & 35 & KDOV RJBB & 5.33249 & 42 & 0 & 0 & 0 & 42 \\
\hline 133 & 1597 & 19 & KDOV $\sim \mathrm{LTAG}$ & 10.73825 & 50.5 & 0 & 0 & 0 & 50.5 \\
\hline 133 & 1597 & 20 & KDOV LTAG & 10.73825 & 0 & 0 & 0 & 4 & 0.8 \\
\hline 133 & 1598 & 46 & KDOV RJBB & 17.30252 & 40.1 & 0 & 0 & 0 & 40.1 \\
\hline 134 & 1608 & 35 & KDOV RJBB & 5.08217 & 42 & 0 & 0 & 0 & 42 \\
\hline 134 & 1609 & 19 & KDOV LTAG & 10.01142 & 50.5 & 0 & 0 & 0 & 50.5 \\
\hline 134 & 1610 & 41 & KBLV RJBB & 12.30845 & 0 & 59.6 & 0 & 0 & 59.6 \\
\hline 135 & 1620 & 35 & KDOV RJBB & 5.33898 & 35.63 & 0 & 0 & 0 & 35.63 \\
\hline 135 & 1621 & 24 & KDOV ETAR & 9.5482 & 0 & 10.5 & 0 & 0 & 10.5 \\
\hline 135 & 1621 & 26 & KDOV $\sim$ ETAR & 9.5482 & 26.2 & 0 & 0 & 0 & 26.2 \\
\hline 135 & 1622 & 52 & KDMA RJBB & 17.84969 & 26.2 & 0 & 0 & 0 & 26.2 \\
\hline 136 & 1632 & 35 & KDOV RJBB & 4.99295 & 42 & 0 & 0 & 0 & 42 \\
\hline 136 & 1633 & 26 & KDOV $\sim$ ETAR & 9.44297 & 29.6 & 0 & 0 & 0 & 29.6 \\
\hline 136 & 1634 & 52 & KDMA RJBB & 18.14858 & 26.2 & 0 & 0 & 0 & 26.2 \\
\hline 137 & 1644 & 35 & KDOV $\sim \mathrm{RJBB}$ & 4.9517 & 7.89 & 0 & 0 & 0 & 7.89 \\
\hline 137 & 1645 & 12 & KBLV LTAG & 9.91389 & 0 & 0 & 34.2 & 0 & 34.2 \\
\hline 137 & 1646 & 52 & KDMA $\sim$ RJBB & 17.97122 & 26.2 & 0 & 0 & 0 & 26.2 \\
\hline 138 & 1656 & 19 & KDOV $\sim \mathrm{LTAG}$ & 10.99965 & 50.5 & 0 & 0 & 0 & 50.5 \\
\hline 138 & 1658 & 52 & KDMA RJBB & 18.19162 & 26.2 & 0 & 0 & 0 & 26.2 \\
\hline 139 & 1668 & 11 & KBLV LTAG & 4.89086 & 16.5 & 46.27 & 0 & 0 & 62.77 \\
\hline 139 & 1669 & 19 & KDOV LTAG & 10.08404 & 50.5 & 0 & 0 & 0 & 50.5 \\
\hline 139 & 1670 & 52 & KDMA RJBB & 17.9638 & 26.2 & 0 & 0 & 0 & 26.2 \\
\hline 140 & 1680 & 11 & KBLV LTAG & 5.29575 & 0 & 47.9 & 0 & 0 & 47.9 \\
\hline 140 & 1682 & 52 & KDMA RJBB & 17.88614 & 26.2 & 0 & 0 & 0 & 26.2 \\
\hline 141 & 1693 & 5 & KDOV $\sim \mathrm{OKBK}$ & 1.26658 & 0 & 30.9 & 0 & 0 & 30.9 \\
\hline 141 & 1694 & 52 & KDMA RJBB & 18.18504 & 26.2 & 0 & 0 & 0 & 26.2 \\
\hline 142 & 1704 & 14 & KDOV LTAG & 6.44895 & 0 & 0 & 34.5 & 0 & 34.5 \\
\hline 142 & 1705 & 27 & KDOV OKBK & 11.86112 & 0 & 0 & 34.2 & 0 & 34.2 \\
\hline 142 & 1706 & 52 & KDMA RJBB & 17.97122 & 26.2 & 0 & 0 & 0 & 26.2 \\
\hline 143 & 1717 & 27 & KDOV OKBK & 11.86152 & 0 & 0 & 31.6 & 0 & 31.6 \\
\hline 143 & 1717 & 33 & KDOV OKBK & 11.86152 & 23.52 & 0 & 0 & 0 & 23.52 \\
\hline 143 & 1718 & 52 & KDMA RJBB & 18.19162 & 20.6 & 0 & 0 & 0 & 20.6 \\
\hline 143 & 1716 & 40 & KDOV $\sim \mathrm{RJBB}$ & 25.3324 & 23.7 & 0 & 0 & 0 & 23.7 \\
\hline 144 & 1728 & 15 & KBLV LTAG & 6.03438 & 0 & 21.83 & 0 & 0 & 21.83 \\
\hline 144 & 1728 & 16 & KBLV LTAG & 6.03438 & 5.6 & 6.7 & 0 & 0 & 12.3 \\
\hline 144 & 1729 & 33 & KDOV OKBK & 12.09925 & 51.4 & 0 & 0 & 0 & 51.4 \\
\hline 144 & 1730 & 44 & KBLV RJBB & 20.78642 & 0 & 10.5 & 0 & 0 & 10.5 \\
\hline 145 & 1740 & 20 & KDOV LTAG & 8.44661 & 0 & 0 & 39.2 & 0 & 39.2 \\
\hline 145 & 1741 & 33 & KDOV OKBK & 12.18031 & 51.4 & 0 & 0 & 0 & 51.4 \\
\hline 145 & 1742 & 48 & KBLV RJBB & 22.23186 & 18 & 0 & 0 & 0 & 18 \\
\hline 146 & 1752 & 3 & KDMA OKBK & 2.1009 & 0 & 0 & 41.4 & 28 & 47 \\
\hline 146 & 1753 & 33 & KDOV OKBK & 12.05465 & 51.4 & 0 & 0 & 0 & 51.4 \\
\hline 146 & 1754 & 34 & KDMA OKBK & 23.0896 & 0 & 14.2 & 0 & 0 & 14.2 \\
\hline 147 & 1766 & 12 & KBLV LTAG & 8.76197 & 0 & 0 & 34.2 & 0 & 34.2 \\
\hline 147 & 1764 & 19 & KDOV LTAG & 11.00444 & 50.5 & 0 & 0 & 17 & 53.9 \\
\hline
\end{tabular}




\begin{tabular}{|c|c|c|c|c|c|c|c|c|c|}
\hline & & & & & \multicolumn{5}{|c|}{-------DELIVERED QUANTITIES-------- } \\
\hline Tail\# & Msn\# & REQT\# & APOE APOD & ARR APOD & OUT & OVER & BULK & PAX & TOTAL \\
\hline 147 & 1765 & 33 & KDOV OKBK & 12.98718 & 21.08 & 0 & 7.9 & 0 & 28.98 \\
\hline 148 & 1776 & 17 & KDMA $\sim \mathrm{OKBK}$ & 8.09098 & 0 & 31.4 & 0 & 0 & 31.4 \\
\hline 148 & 1778 & 40 & KDOV RJBB & 25.59421 & 23.7 & 0 & 0 & 0 & 23.7 \\
\hline 149 & 1788 & 17 & KDMA OKBK & 8.0913 & 0 & 31.4 & 0 & 0 & 31.4 \\
\hline 149 & 1789 & 28 & KDMA LTAG & 13.13221 & 0 & 6.7 & 0 & 7 & 8.1 \\
\hline 149 & 1790 & 40 & KDOV RJBB & 24.99291 & 23.7 & 0 & 0 & 0 & 23.7 \\
\hline 150 & 1800 & 17 & KDMA $\sim \mathrm{OKBK}$ & 8.09011 & 0 & 13.1 & 0 & 0 & 13.1 \\
\hline 150 & 1801 & 19 & KDOV LTAG & 11.14871 & 50.5 & 0 & 0 & 0 & 50.5 \\
\hline 150 & 1802 & 40 & KDOV RJBB & 24.84233 & 23.7 & 0 & 0 & 0 & 23.7 \\
\hline 151 & 1812 & 13 & KDMA LTAG & 9.45659 & 22.3 & 0 & 0 & 0 & 22.3 \\
\hline 151 & 1814 & 40 & KDOV RJBB & 25.40531 & 23.7 & 0 & 0 & 0 & 23.7 \\
\hline 152 & 1824 & 12 & KBLV LTAG & 9.201 & 0 & 0 & 32.83 & 0 & 32.83 \\
\hline 152 & 1824 & 18 & KBLV LTAG & 9.201 & 0 & 0 & 20.3 & 0 & 20.3 \\
\hline 152 & 1824 & 21 & KBLV LTAG & 9.201 & 0 & 10.24 & 0 & 0 & 10.24 \\
\hline 152 & 1825 & 30 & KBLV LTAG & 13.09166 & 0 & 0 & 41.4 & 0 & 41.4 \\
\hline 152 & 1826 & 40 & KDOV RJBB & 25.22797 & 23.7 & 0 & 0 & 0 & 23.7 \\
\hline 153 & 1836 & 21 & KBLV LTAG & 9.02492 & 0 & 47.9 & 0 & 0 & 47.9 \\
\hline 153 & 1837 & 30 & KBLV LTAG & 12.9117 & 0 & 0 & 41.4 & 0 & 41.4 \\
\hline 153 & 1838 & 40 & KDOV $\sim$ RJBB & 25.55775 & 23.7 & 0 & 0 & 0 & 23.7 \\
\hline 154 & 1848 & 21 & KBLV LTAG & 9.05363 & 0 & 47.9 & 0 & 0 & 47.9 \\
\hline 154 & 1849 & 40 & KDOV RJBB & 24.9565 & 23.7 & 0 & 0 & 22 & 28.1 \\
\hline 154 & 1849 & 47 & KDOV $\sim$ RJBB & 24.9565 & 0 & 0 & 0 & 88 & 17.6 \\
\hline 154 & 1850 & 40 & KDOV $\sim \mathrm{RJBB}$ & 27.55366 & 23.7 & 0 & 0 & 0 & 23.7 \\
\hline 155 & 1861 & 38 & KDMA RJBB & 5.14862 & 23.3 & 0 & 37.84 & 49 & 70.94 \\
\hline 155 & 1860 & 21 & KBLV LTAG & 8.9101 & 0 & 4.36 & 0 & 0 & 4.36 \\
\hline 155 & 1860 & 11 & KBLV LTAG & 8.9101 & 0 & 4.43 & 0 & 0 & 4.43 \\
\hline 155 & 1862 & 40 & KDOV $\sim$ RJBB & 24.84233 & 23.7 & 0 & 0 & 0 & 23.7 \\
\hline 156 & 1872 & 19 & KDOV LTAG & 10.84667 & 50.5 & 0 & 0 & 0 & 50.5 \\
\hline 156 & 1873 & 31 & KBLV LTAG & 15.18258 & 0 & 33.6 & 34 & 0 & 67.6 \\
\hline 156 & 1874 & 40 & KDOV $\sim$ RJBB & 25.08213 & 23.7 & 0 & 0 & 0 & 23.7 \\
\hline 157 & 1884 & 30 & KBLV LTAG & 12.76197 & 0 & 0 & 41.4 & 28 & 47 \\
\hline 157 & 1885 & 31 & KBLV LTAG & 15.69874 & 0 & 0 & 41.4 & 0 & 41.4 \\
\hline 157 & 1886 & 40 & KDOV $\sim \mathrm{RJBB}$ & 25.2279 & 17.3 & 0 & 0 & 0 & 17.3 \\
\hline 158 & 1897 & 7 & KDSM LTAG & 2.63139 & 0 & 6.6 & 0 & 0 & 6.6 \\
\hline 158 & 1896 & 30 & KBLV LTAG & 12.47269 & 0 & 0 & 41.4 & 28 & 47 \\
\hline 158 & 1898 & 56 & KDOV $\sim$ RJBB & 25.95649 & 0 & 24.5 & 20 & 0 & 44.5 \\
\hline
\end{tabular}

Table A.4 Scenario 4 Mission Summary

\section{A.5 SCENARIO 5 BEST SOLUTION MISSION SUMMARY}

\begin{tabular}{|l|l|l|l|r|l|l|l|l|l|}
\hline & & & & \multicolumn{3}{|l|}{------ DELIVERED QUANTITIES------- } \\
\hline Tail\# & Msn\# & REQT\# & APOE $\sim$ APOD & ARR APOD & OUT & \multicolumn{1}{l|}{ OVER } & BULK & PAX & TOTAL \\
\hline 0 & 0 & 4 & KDOV OKBK & 0.87393 & 0 & 0 & 0 & 133 & 26.6 \\
\hline 0 & 0 & 5 & KDOV $\sim$ OKBK & 0.87393 & 0 & 0 & 0 & 5 & 1 \\
\hline 0 & 1 & 35 & KDOV RJBB & 5.17778 & 0 & 0 & 0 & 280 & 56 \\
\hline 0 & 3 & 15 & KBLV LTAG & 7.12879 & 0 & 0 & 0 & 335 & 67 \\
\hline 0 & 3 & 16 & KBLV $\sim$ LTAG & 7.12879 & 0 & 0 & 0 & 24 & 4.8 \\
\hline 0 & 2 & 19 & KDOV LTAG & 9.67014 & 0 & 0 & 0 & 322 & 64.4 \\
\hline 1 & 12 & 0 & KBLV LTAG & 0.98996 & 0 & 0 & 0 & 300 & 60 \\
\hline 1 & 13 & 34 & KDMA $\sim$ OKBK & 22.92198 & 0 & 0 & 0 & 133 & 26.6 \\
\hline
\end{tabular}




\begin{tabular}{|c|c|c|c|c|c|c|c|c|c|}
\hline \multirow[b]{2}{*}{ Tail\# } & \multirow[b]{2}{*}{ Msn\# } & \multirow{2}{*}{ REQT\# } & \multirow{2}{*}{ APOE $\sim$ APOD } & \multirow{2}{*}{ ARR APOD } & \multicolumn{5}{|c|}{ 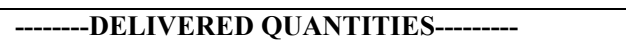 } \\
\hline & & & & & OUT & OVER & BULK & PAX & TOTAL \\
\hline 1 & 13 & 32 & KDMA $\sim \mathrm{OKBK}$ & 22.92198 & 0 & 0 & 0 & 12 & 2.4 \\
\hline 1 & 15 & 37 & KDMA RJBB & 24.79628 & 0 & 0 & 0 & 280 & 56 \\
\hline 2 & 24 & 3 & KDMA OKBK & 1.92282 & 0 & 0 & 0 & 335 & 67 \\
\hline 2 & 25 & 10 & KDMA LTAG & 5.55837 & 0 & 0 & 0 & 146 & 29.2 \\
\hline 2 & 26 & 29 & KDOV LTAG & 11.87848 & 0 & 0 & 0 & 335 & 67 \\
\hline 2 & 27 & 51 & KBLV RJBB & 23.91638 & 0 & 0 & 0 & 123 & 24.6 \\
\hline 3 & 37 & 38 & KDMA RJBB & 4.87719 & 0 & 0 & 0 & 233 & 46.6 \\
\hline 3 & 38 & 29 & KDOV LTAG & 11.76729 & 0 & 0 & 0 & 269 & 53.8 \\
\hline 3 & 36 & 45 & KDMA RJBB & 15.71802 & 0 & 0 & 0 & 120 & 24 \\
\hline 3 & 39 & 37 & KDMA RJBB & 24.71809 & 0 & 0 & 0 & 296 & 59.2 \\
\hline 3 & 39 & 49 & KDMA RJBB & 24.71809 & 0 & 0 & 0 & 63 & 12.6 \\
\hline 4 & 50 & 42 & KDOV RJBB & 14.91798 & 0 & 0 & 0 & 335 & 67 \\
\hline 4 & 50 & 53 & KDOV RJBB & 14.91798 & 0 & 0 & 0 & 24 & 4.8 \\
\hline 4 & 48 & 53 & KDOV RJBB & 17.26863 & 0 & 0 & 0 & 63 & 12.6 \\
\hline 4 & 51 & 54 & KBLV RJBB & 25.03191 & 0 & 0 & 0 & 230 & 46 \\
\hline 10 & 120 & 9 & KBLV LTAG & 4.69576 & 0 & 0 & 0 & 28 & 5.6 \\
\hline 10 & 120 & 11 & KBLV LTAG & 4.69576 & 0 & 0 & 0 & 3 & 0.6 \\
\hline 10 & 121 & 15 & KBLV LTAG & 6.39537 & 0 & 0 & 0 & 107 & 21.4 \\
\hline 10 & 121 & 16 & KBLV LTAG & 6.39537 & 0 & 0 & 0 & 17 & 3.4 \\
\hline 10 & 123 & 58 & KDOV RJBB & 25.74461 & 0 & 0 & 0 & 123 & 24.6 \\
\hline 11 & 133 & 3 & KDMA OKBK & 1.90754 & 0 & 0 & 0 & 195 & 39 \\
\hline 11 & 132 & 7 & KDSM LTAG & 3.81757 & 0 & 0 & 0 & 195 & 39 \\
\hline 11 & 134 & 30 & KBLV LTAG & 11.69588 & 0 & 0 & 0 & 65 & 13 \\
\hline 12 & 145 & 12 & KBLV LTAG & 7.69613 & 0 & 0 & 0 & 195 & 39 \\
\hline 12 & 144 & 12 & KBLV LTAG & 9.3834 & 0 & 0 & 0 & 43 & 8.6 \\
\hline 13 & 156 & 7 & KDSM LTAG & 1.92232 & 0 & 0 & 0 & 195 & 39 \\
\hline 13 & 159 & 12 & KBLV LTAG & 7.69597 & 0 & 0 & 0 & 195 & 39 \\
\hline 13 & 157 & 39 & KBLV RJBB & 15.90185 & 0 & 0 & 0 & 123 & 24.6 \\
\hline 13 & 157 & 57 & KBLV RJBB & 15.90185 & 0 & 0 & 0 & 46 & 9.2 \\
\hline 14 & 168 & 7 & KDSM $\sim$ LTAG & 1.70674 & 0 & 0 & 0 & 195 & 39 \\
\hline 14 & 171 & 10 & KDMA LTAG & 4.89699 & 0 & 0 & 0 & 195 & 39 \\
\hline 14 & 169 & 22 & KDMA ETAR & 7.59349 & 0 & 0 & 0 & 195 & 39 \\
\hline 14 & 170 & 42 & KDOV RJBB & 14.70644 & 0 & 0 & 0 & 37 & 7.4 \\
\hline 14 & 170 & 53 & KDOV RJBB & 14.70644 & 0 & 0 & 0 & 158 & 31.6 \\
\hline 20 & 241 & 10 & KDMA LTAG & 5.47693 & 0 & 0 & 10.2 & 0 & 10.2 \\
\hline 20 & 242 & 24 & KDOV ETAR & 9.50815 & 0 & 0 & 8.9 & 0 & 8.9 \\
\hline 20 & 242 & 26 & KDOV ETAR & 9.50815 & 0 & 0 & 1.2 & 0 & 1.2 \\
\hline 20 & 240 & 36 & KDOV RJBB & 13.13003 & 0 & 0 & 72.2 & 0 & 72.2 \\
\hline 20 & 243 & 53 & KDOV $\sim$ RJBB & 15.25706 & 0 & 0 & 56.9 & 0 & 56.9 \\
\hline 21 & 252 & 4 & KDOV OKBK & 0.91463 & 0 & 0 & 84.08 & 0 & 84.08 \\
\hline 21 & 252 & 6 & KDOV OKBK & 0.91463 & 0 & 0 & 13.42 & 0 & 13.42 \\
\hline 21 & 253 & 38 & KDMA RJBB & 4.67726 & 0 & 0 & 75.67 & 0 & 75.67 \\
\hline 21 & 254 & 29 & KDOV LTAG & 12.03607 & 0 & 0 & 68 & 0 & 68 \\
\hline 22 & 264 & 6 & KDOV OKBK & 0.95014 & 0 & 0 & 87.4 & 0 & 87.4 \\
\hline 22 & 265 & 38 & KDMA $\sim$ RJBB & 5.19094 & 0 & 0 & 69.51 & 0 & 69.51 \\
\hline 22 & 266 & 39 & KBLV RJBB & 15.71651 & 0 & 0 & 5.6 & 0 & 5.6 \\
\hline 22 & 266 & 57 & KBLV RJBB & 15.71651 & 0 & 0 & 56 & 0 & 56 \\
\hline 23 & 276 & 6 & KDOV OKBK & 1.01878 & 0 & 0 & 87.4 & 0 & 87.4 \\
\hline 23 & 277 & 38 & KDMA RJBB & 5.28019 & 0 & 0 & 34.82 & 0 & 34.82 \\
\hline 23 & 278 & 27 & KDOV OKBK & 11.81714 & 0 & 0 & 72.2 & 0 & 72.2 \\
\hline 23 & 279 & 31 & KBLV LTAG & 14.7573 & 0 & 0 & 87.38 & 0 & 87.38 \\
\hline 23 & 279 & 30 & KBLV LTAG & 14.7573 & 0 & 0 & 8.2 & 0 & 8.2 \\
\hline 24 & 289 & 14 & KDOV LTAG & 5.71156 & 0 & 0 & 34.5 & 0 & 34.5 \\
\hline 24 & 290 & 27 & KDOV OKBK & 11.81635 & 0 & 0 & 72.2 & 0 & 72.2 \\
\hline 24 & 288 & 29 & KDOV LTAG & 13.70698 & 0 & 0 & 87.4 & 0 & 87.4 \\
\hline 35 & 422 & 27 & KDOV OKBK & 11.9392 & 0 & 0 & 68.4 & 0 & 68.4 \\
\hline 35 & 420 & 27 & KDOV OKBK & 14.34324 & 0 & 0 & 6.6 & 0 & 6.6 \\
\hline 35 & 420 & 33 & KDOV OKBK & 14.34324 & 46.17 & 0 & 0 & 0 & 46.17 \\
\hline
\end{tabular}




\begin{tabular}{|c|c|c|c|c|c|c|c|c|c|}
\hline & & & & & \multicolumn{5}{|c|}{-------DELIVERED QUANTITIES-------- } \\
\hline Tail\# & Msn\# & REQT\# & APOE $\sim$ APOD & ARR APOD & OUT & OVER & BULK & PAX & TOTAL \\
\hline 35 & 423 & 53 & KDOV $\sim \mathrm{RJBB}$ & 16.78814 & 34.5 & 16.9 & 0 & 71 & 65.6 \\
\hline 35 & 421 & 58 & KDOV RJBB & 25.96998 & 34 & 0 & 0 & 0 & 34 \\
\hline 36 & 432 & 4 & KDOV OKBK & 0.93905 & 13 & 6 & 54.52 & 72 & 87.92 \\
\hline 36 & 434 & 7 & KDSM LTAG & 3.51543 & 0 & 0 & 0 & 196 & 39.2 \\
\hline 36 & 433 & 11 & KBLV LTAG & 6.14988 & 16.5 & 54.66 & 0 & 0 & 71.16 \\
\hline 37 & 444 & 3 & KDMA OKBK & 2.27469 & 0 & 0 & 72.05 & 46 & 81.25 \\
\hline 37 & 446 & 17 & KDMA OKBK & 8.27208 & 0 & 22.7 & 0 & 0 & 22.7 \\
\hline 37 & 445 & 41 & KBLV RJBB & 11.91356 & 0 & 76.34 & 0 & 0 & 76.34 \\
\hline 38 & 456 & 1 & KBLV LTAG & 1.24155 & 6.06 & 0 & 23.5 & 0 & 29.56 \\
\hline 38 & 457 & 11 & KBLV LTAG & 5.47573 & 0 & 43.94 & 0 & 0 & 43.94 \\
\hline 38 & 458 & 26 & KDOV ETAR & 9.67517 & 29.6 & 0 & 0 & 0 & 29.6 \\
\hline 38 & 459 & 43 & KDMA $\sim$ RJBB & 12.36759 & 15.2 & 0 & 0 & 0 & 15.2 \\
\hline 39 & 468 & 8 & KDYS LTAG & 2.32322 & 37.2 & 0 & 0 & 0 & 37.2 \\
\hline 39 & 469 & 35 & KDOV RJBB & 5.12614 & 14.97 & 0 & 0 & 27 & 20.37 \\
\hline 39 & 470 & 23 & KDMA ETAR & 9.84855 & 32 & 0 & 0 & 0 & 32 \\
\hline 39 & 471 & 48 & KBLV RJBB & 22.18779 & 18 & 0 & 0 & 0 & 18 \\
\hline 40 & 481 & 13 & KDMA LTAG & 8.47674 & 22.3 & 0 & 0 & 0 & 22.3 \\
\hline 40 & 482 & 26 & KDOV ETAR & 10.50588 & 34.5 & 0 & 0 & 0 & 34.5 \\
\hline 40 & 480 & 34 & KDMA OKBK & 23.25778 & 0 & 14.2 & 0 & 0 & 14.2 \\
\hline 40 & 483 & 40 & KDOV $\sim \mathrm{RJBB}$ & 25.56914 & 16.6 & 0 & 0 & 45 & 25.6 \\
\hline 40 & 483 & 47 & KDOV RJBB & 25.56914 & 0 & 0 & 0 & 190 & 38 \\
\hline 41 & 492 & 3 & KDMA OKBK & 2.26092 & 0 & 0 & 41.95 & 0 & 41.95 \\
\hline 41 & 495 & 15 & KBLV LTAG & 6.05824 & 0 & 26.8 & 0 & 0 & 26.8 \\
\hline 41 & 495 & 16 & KBLV LTAG & 6.05824 & 5.6 & 6.7 & 0 & 0 & 12.3 \\
\hline 41 & 493 & 20 & KDOV LTAG & 8.27762 & 0 & 0 & 51.2 & 4 & 52 \\
\hline 41 & 494 & 27 & KDOV OKBK & 12.10211 & 0 & 0 & 68.4 & 0 & 68.4 \\
\hline 42 & 506 & 25 & KDMA ETAR & 1.84632 & 0 & 14.5 & 0 & 14 & 17.3 \\
\hline 42 & 504 & 25 & KDMA ETAR & 4.05531 & 0 & 0 & 0 & 106 & 21.2 \\
\hline 42 & 505 & 17 & KDMA OKBK & 8.4176 & 0 & 53.2 & 0 & 0 & 53.2 \\
\hline 42 & 507 & 45 & KDMA RJBB & 16.10455 & 0 & 44.5 & 0 & 0 & 44.5 \\
\hline 43 & 516 & 9 & KBLV LTAG & 2.13205 & 0.5 & 11.2 & 45.5 & 0 & 57.2 \\
\hline 43 & 517 & 19 & KDOV LTAG & 9.99306 & 50.6 & 0 & 0 & 0 & 50.6 \\
\hline 43 & 518 & 33 & KDOV OKBK & 12.46969 & 52.63 & 0 & 7.9 & 0 & 60.53 \\
\hline 43 & 519 & 41 & KBLV RJBB & 15.57839 & 0 & 34.06 & 0 & 0 & 34.06 \\
\hline 44 & 528 & 12 & KBLV LTAG & 8.68336 & 0 & 0 & 52.7 & 54 & 63.5 \\
\hline 44 & 528 & 18 & KBLV LTAG & 8.68336 & 0 & 0 & 17.48 & 2 & 17.88 \\
\hline 44 & 528 & 21 & KBLV LTAG & 8.68336 & 0 & 0 & 0 & 0 & 0 \\
\hline 44 & 530 & 28 & KDMA LTAG & 12.37489 & 0 & 6.7 & 0 & 7 & 8.1 \\
\hline 44 & 531 & 39 & KBLV RJBB & 15.91395 & 6.5 & 6.7 & 0 & 0 & 13.2 \\
\hline 44 & 531 & 57 & KBLV RJBB & 15.91395 & 0 & 20.9 & 0 & 0 & 20.9 \\
\hline 44 & 529 & 52 & KDMA RJBB & 18.71123 & 30.2 & 0 & 0 & 0 & 30.2 \\
\hline 55 & 663 & 3 & KDMA OKBK & 2.10225 & 0 & 0 & 41.4 & 28 & 47 \\
\hline 55 & 660 & 7 & KDSM LTAG & 4.37672 & 0 & 0 & 0 & 102 & 20.4 \\
\hline 55 & 662 & 12 & KBLV LTAG & 8.48319 & 0 & 0 & 34.2 & 18 & 37.8 \\
\hline 55 & 661 & 22 & KDMA ETAR & 10.45128 & 0 & 0 & 0 & 102 & 20.4 \\
\hline 56 & 672 & 7 & KDSM LTAG & 1.90229 & 0 & 0 & 0 & 102 & 20.4 \\
\hline 56 & 675 & 30 & KBLV LTAG & 12.09769 & 0 & 0 & 41.4 & 28 & 47 \\
\hline 56 & 674 & 43 & KDMA $\sim$ RJBB & 14.27905 & 16.8 & 0 & 0 & 0 & 16.8 \\
\hline 56 & 673 & 42 & KDOV $\sim \mathrm{RJBB}$ & 18.18421 & 0 & 0 & 0 & 102 & 20.4 \\
\hline 57 & 684 & 7 & KDSM LTAG & 2.00884 & 0 & 0 & 0 & 102 & 20.4 \\
\hline 57 & 685 & 24 & KDOV ETAR & 9.44297 & 0 & 10.5 & 0 & 0 & 10.5 \\
\hline 57 & 685 & 26 & KDOV ETAR & 9.44297 & 26.2 & 0 & 0 & 0 & 26.2 \\
\hline 57 & 686 & 31 & KBLV LTAG & 14.88935 & 0 & 33.6 & 34.92 & 0 & 68.52 \\
\hline 58 & 698 & 5 & KDOV OKBK & 1.36148 & 0 & 30.9 & 0 & 0 & 30.9 \\
\hline 58 & 698 & 6 & KDOV OKBK & 1.36148 & 0 & 0 & 12.38 & 0 & 12.38 \\
\hline 58 & 696 & 7 & KDSM LTAG & 3.62845 & 0 & 0 & 0 & 102 & 20.4 \\
\hline 58 & 697 & 56 & KDOV RJBB & 26.30258 & 0 & 24.5 & 20 & 0 & 44.5 \\
\hline 59 & 708 & 7 & KDSM LTAG & 2.11362 & 0 & 0 & 0 & 102 & 20.4 \\
\hline
\end{tabular}




\begin{tabular}{|c|c|c|c|c|c|c|c|c|c|}
\hline \multirow[b]{2}{*}{ Tail\# } & \multirow[b]{2}{*}{ Msn\# } & \multirow[b]{2}{*}{ REQT\# } & \multirow[b]{2}{*}{ APOE $\sim$ APOD } & \multirow[b]{2}{*}{ ARR APOD } & \multicolumn{5}{|c|}{--------DELIVERED QUANTITIES--------- } \\
\hline & & & & & OUT & OVER & BULK & PAX & TOTAL \\
\hline 59 & 709 & 12 & KBLV LTAG & 8.20187 & 0 & 0 & 34.2 & 18 & 37.8 \\
\hline 59 & 711 & 37 & KDMA RJBB & 25.14863 & 0 & 0 & 41.4 & 28 & 47 \\
\hline 60 & 722 & 2 & KDOV $\sim \mathrm{LTAG}$ & 1.0753 & 0 & 18.1 & 0 & 0 & 18.1 \\
\hline 60 & 720 & 7 & KDSM LTAG & 3.22431 & 0 & 0 & 0 & 102 & 20.4 \\
\hline 60 & 721 & 12 & KBLV LTAG & 8.06416 & 0 & 0 & 34.2 & 18 & 37.8 \\
\hline 60 & 723 & 37 & KDMA RJBB & 24.85445 & 0 & 0 & 41.4 & 0 & 41.4 \\
\hline 61 & 733 & 0 & KBLV LTAG & 1.15761 & 0 & 33.5 & 0 & 0 & 33.5 \\
\hline 61 & 733 & 1 & KBLV LTAG & 1.15761 & 27.14 & 0 & 0 & 0 & 27.14 \\
\hline 61 & 732 & 7 & KDSM LTAG & 3.38106 & 0 & 0 & 0 & 102 & 20.4 \\
\hline 61 & 734 & 42 & KDOV $\sim \mathrm{RJBB}$ & 15.08209 & 0 & 0 & 0 & 102 & 20.4 \\
\hline 61 & 735 & 37 & KDMA RJBB & 24.74032 & 0 & 0 & 41.4 & 0 & 41.4 \\
\hline 62 & 746 & 4 & KDOV OKBK & 0.96105 & 0 & 0 & 41.4 & 28 & 47 \\
\hline 62 & 744 & 7 & KDSM LTAG & 3.29059 & 0 & 0 & 0 & 102 & 20.4 \\
\hline 62 & 745 & 12 & KBLV LTAG & 7.89087 & 0 & 0 & 0 & 102 & 20.4 \\
\hline 62 & 747 & 37 & KDMA RJBB & 25.14858 & 0 & 0 & 31.2 & 0 & 31.2 \\
\hline 62 & 747 & 49 & KDMA $\sim$ RJBB & 25.14858 & 0 & 0 & 0 & 90 & 18 \\
\hline 63 & 757 & 7 & KDSM LTAG & 1.97533 & 0 & 6.6 & 0 & 1 & 6.8 \\
\hline 63 & 756 & 7 & KDSM LTAG & 4.22835 & 0 & 0 & 0 & 102 & 20.4 \\
\hline 63 & 758 & 36 & KDOV $\sim \mathrm{RJBB}$ & 13.2959 & 0 & 0 & 18.2 & 4 & 19 \\
\hline 63 & 758 & 50 & KDOV $\sim \mathrm{RJBB}$ & 13.2959 & 0 & 0 & 0 & 8 & 1.6 \\
\hline 63 & 759 & 49 & KDMA RJBB & 24.7403 & 0 & 0 & 0 & 77 & 15.4 \\
\hline 64 & 769 & 18 & KBLV LTAG & 8.72185 & 0 & 0 & 2.82 & 0 & 2.82 \\
\hline 64 & 769 & 21 & KBLV LTAG & 8.72185 & 0 & 47.9 & 0 & 0 & 47.9 \\
\hline 64 & 768 & 22 & KDMA ETAR & 10.81496 & 0 & 0 & 0 & 101 & 20.2 \\
\hline 64 & 770 & 42 & KDOV RJBB & 14.95645 & 0 & 0 & 0 & 102 & 20.4 \\
\hline 64 & 771 & 55 & KDMA RJBB & 25.93468 & 0 & 0 & 0 & 18 & 3.6 \\
\hline 65 & 782 & 20 & KDOV LTAG & 7.89544 & 0 & 0 & 39.2 & 0 & 39.2 \\
\hline 65 & 781 & 21 & KBLV LTAG & 10.97587 & 0 & 42.5 & 0 & 0 & 42.5 \\
\hline 66 & 792 & 35 & KDOV $\sim \mathrm{RJBB}$ & 5.30251 & 35.63 & 0 & 0 & 15 & 38.63 \\
\hline 66 & 793 & 30 & KBLV LTAG & 12.19945 & 1.2 & 33.6 & 30.4 & 38 & 72.8 \\
\hline 66 & 794 & 46 & KDOV $\sim$ RJBB & 17.30252 & 40.1 & 0 & 0.16 & 0 & 40.26 \\
\hline 67 & 805 & 38 & KDMA RJBB & 4.7403 & 23.3 & 0 & 0 & 0 & 23.3 \\
\hline 67 & 804 & 15 & KBLV LTAG & 7.02831 & 0 & 62 & 0 & 0 & 62 \\
\hline 67 & 806 & 46 & KDOV $\sim$ RJBB & 16.95658 & 50.2 & 0 & 6.04 & 0 & 56.24 \\
\hline 67 & 807 & 44 & KBLV RJBB & 21.23186 & 0 & 10.5 & 8.9 & 0 & 19.4 \\
\hline 68 & 817 & 53 & KDOV RJBB & 15.296 & 10.9 & 17.7 & 0 & 18 & 32.2 \\
\hline 68 & 818 & 40 & KDOV $\sim$ RJBB & 25.08217 & 23.7 & 0 & 0 & 22 & 28.1 \\
\hline 68 & 818 & 47 & KDOV $\sim \mathrm{RJBB}$ & 25.08217 & 0 & 0 & 0 & 74 & 14.8 \\
\hline 69 & 829 & 30 & KBLV LTAG & 12.3052 & 0 & 0 & 0 & 102 & 20.4 \\
\hline 69 & 830 & 40 & KDOV $\sim$ RJBB & 25.3025 & 23.7 & 0 & 0 & 22 & 28.1 \\
\hline 69 & 830 & 47 & KDOV RJBB & 25.3025 & 0 & 0 & 0 & 45 & 9 \\
\hline
\end{tabular}

Table A.5 Scenario 5 Mission Summary 


\section{References}

Aarts, E.H. \& van Laarhoven, P.J. (1985). Statistical Cooling: A General Approach to Combinatorial Optimization Problems, Philips Journal of Research 40: 193-226.

AFDD 2-6 (1999). Air Mobility: Air Force Doctrine Document 2-6, Part of Joint Publication 3-17, Joint Tactics, Techniques, and Procedures for Air Mobility Operations.

AFDD 2-6.1 (1999). Airlift Operations: Air Force Doctrine Document 2-6.1, Part of Joint Publication 3-17, Joint Tactics, Techniques, and Procedures for Air Mobility Operations.

Baker, S.F., Morton, D.P., Rosenthal, R.E. \& Williams, L.M. (2002). Optimizing Military Airlift, Operations Research 50(4): 582-602.

Balas, E. \& Padberg, M. W. (1970). On the Set Covering Problem, Operations Research 20(6): $1152-1161$.

Balas, E. \& Padberg, M. W. (1976). Set Partitioning: A Survey, SIAM Review 18, (4): $710-760$.

Balinski, M.L. \& Quandt, R.E. (1964). On an Integer Program for a Delivery Problem, Operations Research 12, 300-304. 
Barnes, J. W. \& Carlton, W. B. (1995). Solving the Vehicle Routing Problem with Time Windows Using Reactive Tabu Search, presented at the Fall 1995 INFORMS Conference, New Orleans, LA.

Barnes, J. W. \& Colletti, B. (2000). Linearity in the Traveling Salesman Problem, Applied Mathematics Letters 13(3): 27-32.

Barnes, J.W, \& Colletti, B. (2001). Local Search Structure in the Symmetric Traveling Salesman Problem under a General Class of Rearrangement Neighborhoods, Applied Mathematics Letters 14: 105-108.

Barnes, J.W., Colletti, B, \& Neuway, D. (2002). Using Group Theory and Transition Matrices to Study a Class of Metaheuristic Neighborhoods, European Journal of Operational Research 138: 531-544.

Barnes, J.W., Dimova, B., Dokov, S. \& Solomon, A. (2002). The Theory of Landscapes, to appear in Applied Mathematics Letters.

Barnes, J.W., Dimova, B., Solomon, A. \& Dokov, S. (2002). Extending Elementary Landscape Characterizations for Combinatorial Optimization Problems to Arbitrary Neighborhood Definitions, Technical Report Series ORP02-02, The University of Texas at Austin, Graduate Program in Operations Research.

Barnes, J .W., Dokov, S., Acevedo, R. \& Solomon, A. (2002a). A Note on Distance Matrices Yielding Elementary Landscapes for the TSP, to appear in Journal of Mathematical Chemistry. 
Barnes, J.W., Dokov, S., Acevedo, R. \& Solomon, A. (2002b). Weakly Symmetric Distance Matrices and the Traveling Salesman Problem, to appear in Applied Mathematics Letters.

Barnes, J.W., Wiley, V.D., Moore, J.T. \& Ryer, D. (2004). Solving the Aerial Fleet Refueling Problem using Group Theoretic Tabu Search, Mathematical and Computer Modeling 39(6-8): 617-640.

Bartak, R. (1999). Constraint Programming: In Pursuit of the Holy Grail, in Proceedings of WDS99, Prague, Czechkoslovakia.

Battiti, R. and Tecchiolli, G. (1994). The Reactive Tabu Search, ORSA Journal on Computing 6(2): 126-141.

Belenguer, J. M., Martinez, M. C. \& Mota, E. (2000). A Lower Bound for the Split Delivery Vehicle Problem, Operations Research: 48, (5): 801 - 810.

Blanton, J.L. Jr., \& Wainwright, R.L. (1993). Multiple Vehicle Routing with Time and Capacity Constraints using Genetic Algorithms, in S. Forrest, (ed.), Proceedings of the Fifth International Conference on Genetic Algorithms, Morgan Kaufmann Publisher, San Mateo, California, 452 -- 459.

Bloch, N.J. (1987). Abstract Algebra with Applications, Prentice-Hall, Inc., Englewood Cliffs, New Jersey 07632.

Bodin, L. D. (1990). Twenty Years Of Routing And Scheduling, Operations Research: 38, (4): 571 - 579. 
Bodin, L., B. Golden, A. Assad, \& M. Ball. (1983). Routing and Scheduling of Vehicles and Crews; The State of The Art, Computers and Operations Research, 10, 63-211.

Bramel, J.B. \& Simchi-Levi D. (1995). A Location Based Heuristic for General Routing Problems, Operations Research: 43, 649-660.

Bramel, J.B. \& Simchi-Levi,D. (1997). On the Effectiveness of Set Covering Formulations for the Vehicle Routing Problem with Time Windows, Operations Research: 45, 295-301.

Braysy, O. \& Gendreau, M. (2001a). Tabu Search Heuristics for the Vehicle Routing Problem with Time Windows, Internal Report STF42 A01022, SINTEF Applied Mathematics, Department of Optimisation, Oslo, Norway.

Braysy, O. \& Gendreau, M. (2001b). Genetic Algorithms for the Vehicle Routing Problem with Time Windows, Internal Report STF42 A01021, SINTEF Applied Mathematics, Department of Optimisation, Oslo, Norway.

Callander,B.D. (1998). The Evolution of Air Mobility, Air Force Magazine Online, Journal of the Air Force Association 81(2). (http://www.afa.org/magazine/Feb1998/0298evolution.html).

Carlton, W. B. (1995) A Tabu Search Approach to the General Vehicle Routing Problem. Ph.D. thesis, University of Texas, Austin, TX.

Caseau, Y. \& Laburthe, F. (1999). Heuristics for Large Constrained Vehicle Routing Problems, Journal of Heuristics 5: 281-303. 
Chiang, W.C. \& Russell, R. A. (1996). Simulated Annealing Metaheuristics for the Vehicle Routing Problem with Time Windows, Annals of Operations Research: 63, 3-27.

Chiang, W.C. \& Russell, R.A. (1997). A Reactive Tabu Search Metaheuristic for the Vehicle Routing Problem with Time Windows, INFORMS Journal on Computing 9: 417-430.

Christofides, N. \& Beasley, J. (1984). The Period Routing Problem, Networks 14: 237-246.

Christofides, N., Mingozzi, A. \& Toth, P. (1979). The Vehicle Routing Problem. In N. Christofides, A. Mingozzi, P. Toth, \& C. Sandi (eds), Combinatorial Optimization. Wiley, Chichester.

Clarke, G. \& Wright, J.W. (1964). Scheduling of Vehicles from a Central Depot to a Number of Delivery Points, Operations Research 12: 568-581.

Colletti, B. W. (1999). Group Theory and Metaheuristics, PhD thesis, The University of Texas, Austin, TX.

Colletti, B.W. \& Barnes, J.W. (1999a). Group Theory and Metaheuristic Search Neighborhoods, Technical Report Series ORP99-02, Graduate Program in Operations Research and Industrial Engineering, The University of Texas at Austin. 
Colletti, B.W. \& Barnes, J.W. (1999b). Using Group Theory to Efficiently Compute Move Values for Routing Problems. Technical Report Series ORP99-05, Graduate Program in Operations Research, The University of Texas at Austin.

Colletti, B.W., Barnes, J.W. \& Dokov, S. (1999). A Note on Characterizing the k-OPT Neighborhood Via Group Theory, Journal of Heuristics 5(1): 4751.

Combs, T. (2002). A Combined Adaptive Tabu Search and Set Partitioning Approach for the Crew Scheduling Problem with an Air Tanker Crew Application, Ph.D thesis, Air Force Institute of Technology, WrightPatterson AFB, OH.

Combs, T.E \& Moore, J.T. (2004). A Hybrid Tabu Search/Set Pertitioning Approach to Tanker Crew Scheduling, Military Operations Research 9(1): 43-56.

Cook, W. \& Rich, J.L. (1999). A Parallel Cutting-Plane Algorithm for the Vehicle Routing Problems with Time Windows, Working Paper, Department of Computational and Applied Mathematics, Rice University, Houston.

Cordeau, J.F., Laporte, G. \& Mercier, A. (2001). A Unified Tabu Search Heuristic for Vehicle Routing Problems with Time Windows, Journal of the Operational Research Society 52: 928-936.

Crino, J. (2002). A Group Theoretic Tabu Search Methodology for Solving the Theater Distribution Vehicle Routing and Scheduling Problem, Ph.D thesis, Air Force Institute of Technology, Wright-Patterson AFB, OH. 
Crino, J.R., Moore, J.T., Barnes, J.W. \& Nanry, W.P. (2004). Solving the Military Theater Distribution Vehicle Routing and Scheduling Problem Using Group Theoretic Tabu Search, Mathematical and Computer Modeling 39(6-8): 599-616

De Backer, B., Furnon, V. \& Shaw, P. (1999). An Object Model for MetaHeuristic Search in Constraint Programming, Workshop on Integration of AI and OR Techniques in Constraint Programming for Combinatorial Optimization Problems (CP-AI-OR 1999), Ferrera, Italy.

Derigs, U. \& Grabenbauer, G. (1993). INTIME - A New Heuristic Approach to the Vehicle Routing Problem with Time Windows with a Bakery Fleet Case, American Journal of Mathematical and Management Sciences 13: 249-266.

Desrochers, M., Desrosiers, J. \& Solomon, M.M. (1992). A New Optimization Algorithm for the Vehicle Routing Problem with Time Windows, Operations Research: 40, 342-354.

Eglese, R.W. (1990). Simulated Annealing: A Tool for Operational Research, European Journal of Operational Research: 46, 271-281.

Fisher, M.L. \& Jaikumar, R. (1981). A generalized assignment heuristic for vehicle routing, Networks: 11, 109-124.

Focacci, F., Lodi,A. \& Milano, M.(2002). Mathematical Programming Techniques in Constraint Programming: A Short Overview, Journal of Heuristics, 8: 7-17. 
Gaglione, A. M. (1992). An Introduction to Group Theory, Technical Report PU/NRL/5350—92-231, Naval Research Laboratory, Washington, D. C.

Gallian, J.A. (1998). Contemporary Abstract Algebra, Houghton Mifflin Compnay, Boston, MA 02116.

Gaskell, T.J. (1967). Bases for Vehicle Fleet Scheduling, Operational Research Quarterly 18: 218.

Gehring, H. \& Homberger, J. (1999). A Parallel Hybrid Evolutionary Metaheuristic for the Vehicle Routing Problem with Time Windows, in Proceedings of EUROGEN99 - Short Course on Evolutionary Algorithms in Engineering and Computer Science, Reports of the Department of Mathematical Information Technology, Series A. Collections, No. A 2/1999, K. Miettinen, M. Mäkelä \& J. Toivanen (eds), 57-64, University of Jyväskylä, Finland.

Gehring, H. \&. Homberger, J. (2001). Parallelization of a Two-Phase Metaheuristic for Routing Problems with Time Windows, Asia-Pacific Journal of Operational Research 18: 35--47.

Gendreau, M. (2002). Constraint Programming and Operations Research: Comments from an Operations Researcher, Journal of Heuristics 8: 19-24.

Gillett, B.E. \& Miller, L.R. (1974). A heuristic algorithm for the vehicle dispatch problem, Operations Research: 22, 240-349. 
Glover, F. (1989). Tabu Search - Part I, ORSA Journal on Computing 1(3): 190206.

Glover, F. (1990). Tabu Search - Part II, ORSA Journal on Computing 2(1): 432.

Glover, F. \& Laguna, M. (1997) Tabu Search. Kluwer Academic Publishers, Boston, MA.

Glover, F., Laguna, M. \& Marti, R. (2000). Scatter Search and Path Relinking: Foundations and Advance Designs, Leeds School of Business, University of Colorado, Boulder Colorado.

Goldberg, D.E. (1989). Genetic Algorithms in Search, Optimization and Machine Learning, Addison-Wesley, Reading, Massachusetts.

Golden, B.L., Assad, A., Levy, L. and Gheysens, F. (1984), The fleet size and mix vehicle routing problem, Computers and Operations Research 11, 4966.

Golden, B.L., Magnanti, T.L. \& Nguyen, H.Q. (1977). Implementing Vehicle Routing Algorithms, Networks 7: 113-148.

Gomory, R. (1963). An Algorithm for Integer Solutions to Linear Programs, in Recent Advances in Mathematical Programming (eds. Graves \& P. Wolf), McGraw-Hill: New York. 
Gomory, R. (1965). On the Relation Between Integer and Noninteger Solutions to Linear Programs, in Proceedings of the National Academy of Sciences 53(2): 260-265.

Gomory, R. (1967). Faces of an Integer Polyhedron, in Proceedings of the National Academy of Sciences 57(1): 16-18.

Gomory, R. (1969). Some Polyhedra Related to Combinatorial Problems, in Journal of Linear Algebra and its Applications 2(4): 451-558.

Harvey, W.D. \& Ginsberg, M.L. (1995). Limited Discrepancy Search, in Proceedings of the International Joint Conference on Artificial Intelligence (IJCAI) 1: 607-613.

Herstein, I.N. (1975). Topics in Algebra, John Wiley \& Sons, New York.

Hilliard, M. R., Solanki, R. S., Busch, I. K., Harrison, G. \& Kraemer, R. D. (1992). Scheduling the Operation Desert Storm Airlift: An Advanced Automated Scheduling Support System, Interfaces 22(1): 131-146.

ILOG. (2001). ILOG Optimization Suite: Delivering a Competitive Advantage White Paper, ILOG, Gentilly, France. (http://www.ilog.com/products/optimization/tech/optimization_whitepaper .pdf)

The Joint Staff Director of Logistics. (2001). Mobility Requirements Study 2005, U.S. Department of Defense, Washington, D.C. 
Kelly, J.P. \& Xu, J. (1995). Tabu Search and Vocabulary Building for Routing Problems, Graduate School of Business Administration, University of Colorado at Boulder.

Kolen A. \& Pesch, E. (1994). Genetic Local Search in Combinatorial Optimization, Discrete Applied Mathematics, 48: 273-284.

Kontoravdis, G. \& Bard, J. F. (1995). A GRASP for the Vehicle Routing Problem with Time Windows, ORSA Journal on Computing 7: 10-23.

Koulmas, C., Antony, S.R. \& Jaen, R. (1994). A Survey of Simulated Annealing Applications to Operations Research Problems, Omega International Journal of Management Science 22(1): 41-56.

Laporte, G., M. Gendreau, J.Y. Potvin \& F. Semet. (2000). Classical And Modern Heuristics For The Vehicle Routing Problem, International Transactions in Operational Research 7: 285-300.

Laporte, G., \& Nobert, Y. (1987), Exact Algorithms for the Vehicle Routing Problem, Annals of Discrete Mathematics, 31, 147-184.

Larsen, J. (1999). Parallelization of the Vehicle Routing Problem with Time Windows, Ph.D. thesis, Institute of Mathematical Modeling, Technical University of Denmark, Lyngby, Denmark.

Lau, H.C. \& Liang, Z. (2001). Pickup and Delivery with Time Windows : Algorithms and Test Case Generation, in Proceedings of the 13th IEEE International Conference on Tools with Artificial Intelligence (ICTAI'01), Dallas, TX, 333-340. 
Lenstra, J. K. \& Rinnooy Kan, A. H. G. (1981). Complexity of Vehicle Routing and Scheduling Problems, Networks 11: 221-227.

Li, H. \& Lim, A. (2001). A Metaheuristic for the Pickup and Delivery Problem with Time Windows, Proceedings of the 13th IEEE International Conference on Tools with Artificial Intelligence (ICTAI'01), Dallas, TX, 160-170.

Lin, S. (1965). Computer Solutions of the Traveling Salesman Problem, Bell System Technical Journal 44: 2245-2269.

Loeb, V. (2002). In War Effort, U.S. Relies on Strategic Airlifts Air Mobility Fleet Flies Cargo, Troops to World's Tight Spots, Washington Post, June 24, A13.

Lustig, I.J. \& Puget, J.F. (2001). Program Does Not Equal Program: Constraint Programming and Its Relationship to Mathematical Programming, Interfaces 31(6): 29-53.

Meseguer, P. (1997). Interleaved Depth-First Search, in Proceedings of the International Joint Conference on Artificial Intelligence (IJCAI) 2: 13821387.

Moin, N. H. (2002). Hybrid Genetic Algorithms for Vehicle Routing Problems with Time Windows, International Journal of the Computer, the Internet and Management 10(1): 41-57. 
Morton, D., Rosenthal, R. \& Weng, L.T. (1996). Optimization Modeling for Airlift Mobility, Military Operations Research 1(4): 49-67.

Nanry, W. B. (1998). Solving the Precedence Constrained Vehicle Routing Problem with Time Windows Using the Reactive Tabu Search Metastrategy, PhD thesis, The University of Texas at Austin.

Nanry, W. P. \& Barnes, J. W. (2000). Solving the Pickup and Delivery Problem with Time Windows Using Reactive Tabu Search, Transportation Research Part B 34: 107-121.

Nelson, M.D., Nygard, K.E., Gri.n, J.H. \& Shreve, W.E. (1985). Implementation Techniques for the Vehicle Routing Problem, Computers \& Operations Research 12: 273-283.

Nilsson, N.J. (1971). Problem Solving Methods in Artificial Intelligence, McGraw-Hill, New York.

Or, I. (1976). Traveling Salesman-type Combinatorial Problems and their Relation to the Logisitics of Blood Banking, Ph.D. thesis, Northwestern University, Evanston, IL.

Osman, I.H. (1993). Metastrategy Simulated Annealing and Tabu Search Algorithms for Vehicle Routing Problems, Annals of Operations Research 40: 421-452.

Osman, I.H. \& Kelly, J.P. (eds.). (1996). Meta-Heuristics: Theory and Applications, Kluwer Academic Publishers, Boston. 
Paessens, H. (1988). The Savings Algorithm for the Vehicle Routing Problem, European Journal of Operational Research 34: 336-344.

Pesant, G., Gendreau, M. \& Roousseau, J.M. (1997). GENIUS-CP: A Generic Single-Vehicle Routing Algorithm, Principles and Practice of Constraint Programming: Proceedings of the Third International Conference (CP '97): 420-434.

Potvin, J.Y., Kervahut, T., Garcia, B.L. \& Rousseau, J.M. (1996). The Vehicle Routing Problem With Time Windows - Part I : Tabu Search, INFORMS Journal of Computing 8: 158 - 164 .

Potvin, J.Y. \& Rousseau, J.M. (1995). An Exchange Heuristic for Routing Problems with Time Windows, Journal of the Operational Research Society 46: 1433-1446.

Rappoport, Harold K., Levy, Laurence S., Golden, Bruce L. \& Feshbach, David S. (1991). Estimating Loads of Aircraft in Planning for the Military Airlift Command, Interfaces 21(4): 63-78.

Rappoport, Harold K., Levy, Laurence S., Golden, Bruce L. \& Toussaint, Katherine J. (1992). A Planning Heuristic for Military Airlift, Interfaces 22(3): 73-87.

Rego, C. (1998). A Subpath Ejection Method for the Vehicle Routing Problem, Management Science 44(10): 1447-1459. 
Renaud J., Boctor, F.F. \& Laporte, G. (1996). A Fast Composite Heuristic for the Symmetric Traveling Salesman Problem, INFORMS Journal on Computing 8: 134--143.

Renaud,J., Boctor, F.F. \& Ouenniche, J. (2000). A Heuristic for the Pickup and Delivery Traveling Salesman Problem, Computers \& Operations Research 27: $905-916$.

Rochat, Y. \& Semet, F. (1994). A Tabu Search Approach for Delivering Pet Food and Pet Flour in Switzerland, Journal of the Operational Research Society 45(11): 1233-1246.

Rosenthal, R.E., Baker, S.F., Weng, L.T., Fuller, D.F., Goggins, D., Toy, A.O., \& Turker, Y. (1997). Application and Extension of the Thruput II Optimization Model for Airlift Mobility, Military Operations Research 3(2): 55-74.

Rousseau, L.M., Gendreau, M. \& Peseant, G. (2002). Using Constraint-Based Operators to Solve the Vehicle Routing Problem with Time Windows, Journal of Heurisitcs 8: 43-58.

Rower, T.I. (2001). An Overview and Assessment of U.S. Strategic Airlift, Strategy Research Project, U.S. Army War College, Carlisle Barracks, PA.

Russell, R.A. (1995). Hybrid Heuristics for the Vehicle Routing Problem with Time Windows, Transportation Science 29: 156-166.

Shaw, P. (1998). Using Constraint Programming and Local Search Methods to Solve Vehicle Routing Problems, in 4th International Conference on 
Principles and Practices of Constraint Programming (CP '98), Lecture Notes in Computer Science 1520: 417-431.

Solomon, M. M. (1987). Algorithms for the Vehicle Routing and Scheduling Problems with Time Window Constraints, Operations Research 35(2): $254-265$.

Taillard, E., Badeau, P., Gendreau, M., Guertin, F. \& Potvin, J. (1997). A Tabu Search Heuristic for the Vehicle Routing Problem with Soft Windows, Transportation Science 31: 170-186.

Tan, K.C., Lee, L.H. \& Zhu, K.Q. (2000). Heuristic Methods for Vehicle Routing Problem with Time Windows, in Proceedings of the 6th International Symposium on Artificial Intelligence \& Mathematics, Ft. Lauderdale, Florida.

Thangiah, S.R., 1995, "Vehicle Routing with Time Windows using Genetic Algorithms", in L. Chambers, (ed), Practical Handbook of Genetic Algorithms: New Frontiers, II, pp 253 --277.

Thangiah, S.R., Nygard, K.E., \& Juell, P.L., 1991, "GIDEON: A Genetic Algorithm System for Vehicle Routing with Time Windows", Proceedings Seventh IEEE Conference on Artificial Intelligence Applications, IEEE Computer Society press, Los Alamitos, California, pp $332--325$.

Walsh, T. (1997). Depth-Bounded Discrepancy Search, in Proceedings of the International Joint Conference on Artificial Intelligence (IJCAI) 2: 13881393. 
Wiley, V.D. (2001). The Aerial Fleet Refueling Problem. Ph.D. thesis, University of Texas, Austin, TX.

Williams, E. (2002). Aviation Formulary V1.37

( http://williams.best.vwh.net/avform.htm )

Wolsey, L. (1971a). Extensions of the Group Theoretic Approach in Integer Programming, Management Science 18(1): 74-83.

Wolsey, L. (1971b). Group Theoretic Results in Mixed Integer Programming, Operations Research 19(7): 1691-1697.

Yellow, P. (1970). A Computational Modification to the Savings Method of Vehicle Scheduling, Operational Research Quarterly 21: 281-283.

Yost, K. A. (1994). The THRUPUT Strategic Airlift Flow Optimization Model, Air Force Studies and Analyses Agency, The Pentagon, Washington, D.C. 


\section{Vita}

Garrett Randall Lambert was born in Topeka, Kansas on April 12 $2^{\text {th }}, 1962$, the son of Henry Dillon and Joan Randall Lambert. A 1980 graduate of Iona Preparatory High School, New Rochelle, New York, he entered the United States Military Academy in West Point, New York. He received a B.S. from the United States Military Academy in May 1984 and was commissioned as a second lieutenant in the Infantry. His military service has been continuous to the present day and he has held positions as Rifle Platoon Leader, Company Executive Officer, Battalion Logistics Officer (S4), Brigade and Battalion Assistant Operations Officer (Asst. S3), Company Commander, Instructor and Assistant Professor in the Department of Mathematical Sciences at West Point, Battalion Executive Officer, and Corps Assistant Operations Officer for Training (Asst. G3 Training). He currently holds the rank of Lieutenant Colonel. He received a M.S. in Industrial Engineering from Texas A\&M University, College Station, Texas in May 1995. He received a M.A. in National Security and Strategic Studies from the Naval War College, Newport, Rhode Island in June 1998. In June 2000 he began doctoral studies in the Operations Research, Industrial Engineering Program at the University of Texas, Austin, Texas.

Permanent address: 211E Barry Road, West Point, NY 10996

This dissertation was typed by the author. 